\title{
gynécologie
} Société Suisse de Gynécologie et d'Obstétrique Schweizerische Gesellschaft für Gynäkologie und Geburtshilfe Società Svizzera di Ginecologia e Ostetricia

\section{Jahresversammlung und Kongress Assemblée annuelle et congrès Assemblea annuale e congresso}

Lugano, 23.-25.6.2005

\section{Freie Mitteilungen}

\section{FM I/10 Geburtshilfe \\ Die fetale Sinusbradykardie: Risikofaktor für SIDS (Sudden Infant Death Syndrome)?}

Beinder E.

Klinik für Geburtshilfe, Universitätsspital Zürich

Einführung: SIDS (sudden infant death syndrome) ist in Industrieländern die häufigste Todesursache von Kindern im Alter von 1 bis 6 Monaten. Schwartz et al. [NEJM 1998;338:1709] beschrieben eine starke Assoziation zwischen SIDS (sudden infant death syndrome) und Long-QT-Syndromen (vererbte Repolarisationsstörungen des Herzens) bei Neugeborenen. Anhand der Auswertung von Schwangerschafts- und Geburtsbefunden von Kindern mit postpartal nachgewiesenem Long-QT-Syndrom (LQTS) untersuchten wir, ob bereits pränatal bei diesen Kindern Auffälligkeiten nachweisbar waren. Methodik: Wir identifizierten 18 Kinder aus Einlingsschwangerschaften, die in Terminnähe geboren und bei denen nach der Geburt oder in der Kindheit ein LQTS diagnostiziert wurde. Von 17 dieser 18 Kinder waren der Schwangerschafts- und Geburtsverlauf, sowie die Befunde von Ultraschalluntersuchungen und das CTG während der Entbindung verfügbar. Ergebnisse: Im CTG war bei 12 von 17 Feten eine permanente Sinusbradykardie (basale Herzfrequenz <120/min) nachweisbar (71\%). Sinusbradykardien sind ansonsten bei Termingeburten in weniger als 3\% der Fälle vorhanden. 2 Feten wiesen zusätzlich intermittierende tachykarde Rhythmusstörungen auf. Einer dieser Feten war aufgrund einer dekompensierten Herzinsuffizienz hydropisch. Beide Kinder mit Herzrhythmusstörungen verstarben trotz intensivmedizinischer Behandlung noch am ersten Lebenstag. Ein weiteres Kind starb plötzlich und unerwartet im Alter von 3 Wochen. Keines der 17 Kinder wies nach den Apgarwerten und/oder dem Nabelarterien-pH eine peripartale Asphyxie auf. Alle 3 verstorbenen Kinder waren männlich und wiesen die längsten QT-Intervalle $(0,64$ 0,67 s) auf. Schlussfolgerung: Anhaltende fetale Sinusbradykardien können ein Hinweis für ein LQTS des Feten sein und somit ein erhöhtes Risiko für ein SIDS darstellen. Postpartal sollte bei diesen Kindern ein EKG angefertigt werden, um ein LQTS auszuschliessen.

\section{FM 1/11 Geburtshilfe \\ Outcome von Zwillingsschwangerschaften mit singulärer Nabelschnurarterie}

Frei K.A., Dürig P., Kuhn P., Fässler S., Waber H., Schneider H., Raio $L$.

Universitäts-Frauenklinik, Inselspital Bern

Einführung: Die singuläre Umbilikalarterie (SUA) ist eine der häufigsten Anomalien beim Menschen mit einer Inzidenz von bis zu 1\%. Fehlbildungen, Wachstumsretardierung (IUWR) als auch Plazentaanomalien sind damit assoziiert. Ziel dieser Studie ist es, Inzidenz und assoziierten Probleme bei Zwillingsschwangerschaften (ZS) zu analysieren. Material und Methoden: Alle konsekutiven ZS mit vollständiger postpartaler Information wurden in die Studie eingeschlossen. Die ZS wurden als monochorial beurteilt, wenn eine gemeinsame Plazenta mit T-Zeichen vorlag, als dichorial wenn ein lambda-Zeichen oder getrennte Placentae vorlagen. Eine IUWR lag bei einem songraphischen Abdomenumfang unter der 5. Perzentile vor, Untergewicht für das Gestationsalter (SGA) wurde bei einem Geburtsgewicht unter der 10. Perzentile diagnostiziert. Ein diskordantes Geburtsgewicht wurde bei einer Gewichtsdifferenz von mehr als 20\% festgelegt. Die Diagnose der SUA wurde sonographisch gestellt und postpartal makroskopisch oder histologisch bestätigt. Resultate: Es konnten 170 ZS mit einem medianen Gestationsalter von 26 Wochen $(18-38,2)$ in die Studie eingeschlossen werden. Bei 16 (9,4\%) Zwillingsschwangerschaften wurde bei mindestens einem Zwilling eine SUA diagnostiziert, davon waren $6(8.3 \%)$ monochorial und $10(10,2 \%)$ dichorial $(p=n . s$.$) .$

\section{KARGER}

() 2005 S. Karger AG, Basel

Fax +4161306 1234 
Die Prävalenz von IUWR, SGA und diskordantem Geburtsgewicht war signifikant höher in den ZS mit SUA [IUWR: 28,1 vs $12,7 \%$; $p<0.05$. SGA: 37,5 vs. $20,1 \%$; $p<0,05$, diskordantes Geburtsgewicht 66,7 vs. $28,6 \%$; $<<0.01$ ]. Schlussfolgerung: ZS mit SUA bei mindestens einem Zwilling haben ein höheres Risiko für IUWR, SGA und diskordantes Geburtsgewicht verglichen mit ZS ohne SUA. Die ähnliche Prävalenz von SUA bei monochorialen und dichorialen ZS lässt postulieren, dass Umweltfaktoren eine wichtigere Rolle in der Pathogenese dieser Nabelschnurauffälligkeit spielen als genetische Faktoren.

\section{FM I/12 Geburtshilfe \\ Produktion von C reaktivem Protein in Plazentagewebe am Termin}

Raio L. ${ }^{a}$, Malek A. ${ }^{a}$, Mueller M.D. ${ }^{a}$, Di Santo S. ${ }^{\text {, }}$ Bersinger N. ${ }^{\mathrm{a}}$, Ghezzi $\mathrm{F}^{\mathrm{b}}$, Passi A. ${ }^{\mathrm{c}}$, Schneider $\mathrm{H}^{\mathrm{a}}$

aniversitäts-Frauenklinik Inselspital Bern,

bUniversitäts-Frauenklinik Universität Varese,

'Department für experimentelle und klinische Biochemie, Universität Varese

Einführung: $\mathrm{C}$ reaktives Protein (CRP) wurde auch im Fruchtwasser gefunden und erhöhte Werte waren mit einem ungünstigen Ausgang der Schwangerschaft assoziiert. Die folgende Studie hatte zum Ziel, mittels in vivo und in vitro Experimenten herauszufinden, ob Plazentagewebe fähig ist, CRP zu synthetisieren und in welchem Ausmass CRP im fetalen oder mütterlichen Kreislauf akkumuliert. Material und Methoden: Zehn gepaarte Blutproben aus peripherem mütterlichem Blut (MB) und Blut getrennt aus Nabelschnurvene (NSV) - und -arterien (NSA) wurden im Rahmen von elektiven Sectiones am Termin gesammelt. Diese in vivo Untersuchungen wurden durch in vitro Experimente (Plazenta-Perfusionsmodell, ELISA, real time PCR) ergänzt. Resultate: Die mediane CRP-Konzentration war in der NSV signifikant höher als in der NSA \&\#61531; 50,1 ng/ml $(12,1-684,6)$ vs. $61 \mathrm{ng} / \mathrm{ml}(16,9-$ 708,1) \&\#61533. Beide Werte korrelierten signifikant mit denjenigen im MB. Im Perfusionsmodell konnte eine aktive de novo Synthese von CRP nachgewiesen werden, wobei nur 0,59\% im fetalen Kompartiment akkumulierte. Dieser Wert war vergleichbar mit den Resultaten aus den in vivo Experimenten (0,35\%). Mittels real time PCR konnte in Plazentagewebe eine CRP Genexpression nachgewiesen werden. Schlussfolgerung: Plazentagewebe ist fähig, unter in vitro Bedingungen CRP zu synthetisieren. 99,41\% des Proteins akkumuliert im mütterlichen Kompartiment. Weitere Studien sind nötig, um die Rolle des plazentaren CRP im Rahmen der Schwangerschaft zu untersuchen.

\section{FM I/13 Geburtshilfe \\ Depistage de la colonisation maternelle par le streptocoque du groupe B et $E$. coli lors du travail: développement d'un test $P C R$ rapide}

Stan C. ${ }^{\mathrm{a}}$, Boulvain M. ${ }^{\mathrm{a}}$, Renzi G. ${ }^{\mathrm{b}}$, Rohner $\mathrm{P}^{\mathrm{b}}$, Schrenzel J. ${ }^{\mathrm{b}}$, Irion O. $^{a}$

aDépartement d'Obstétrique et de Gynécologie, 'baboratoire Central de Bactériologie, Hôpitaux Universitaires de Genève

Introduction: Le streptocoque du groupe B (GBS) et Escherichia coli (E. coli) sont les principales causes de morbidité et mortalité infectieuses du nouveau-né. La prophylaxie antibiotique lors du travail diminue de $90 \%$ le risque de transmission verticale du GBS, mais le dépistage des femmes colonisées pendant le travail est limité par le résultat tardif des cultures génitales. Le dépistage anténatal par culture a une sensibilité mauvaise dans notre contexte épidémiologique. Les tests rapides disponibles ont une sensibilité et spécificité décevante. Notre équipe multidisciplinaire a mis au point un test rapide basé sur PCR pour le dépistage simultané du GBS et $E$. coli (K1). Méthode: Les cibles ADN spécifiques pour le GBS étaient disponibles. Concernant le $E$. coli et le E. coli K1, des séquences $\mathrm{ADN}$ spécifiques ont été identifiées après l'analyse génomique des souches invasives disponibles dans des anciennes cultures prélevées chez des nouveau-nés présentant un sepsis néonatal ou une méningite avec $E$. coli $(\mathrm{K} 1)$. La spécificité des sondes $\mathrm{ADN}$ a été testée en rapport avec multiples bactéries parentés ou non-parentés et ensuite en clinique sur un échantillon de femmes enceintes. Les caractéristiques des sondes sélectionnées ont été testées dans une étude pilote sur 200 femmes en travail. Résultats: La prévalence de la colonisation maternelle par GBS, E. coli agressif et $E$. coli $\mathrm{K} 1$ a été de 15, 6,5 et 3,5\% respectivement. En comparaison avec la culture, considérée comme gold standard, la sensibilité des tests PCR pour le GBS, E. coli et E. coli K1 était de 96,5, 39 et $71 \%$ respectivement. La spécificité était de 88,91 et $94 \%$. La durée moyenne de l'analyse PCR était $2 \mathrm{~h}$. Conclusion: Ce test PCR rapide a une bonne fiabilité pour le dépistage du GBS et pourrait être intéressant en usage clinique, car un résultat rapide et fiable permet une prophylaxie antibiotique ciblée et précoce. L'optimisation en cours du test pour $E$. coli $\mathrm{K} 1$ pourrait améliorer sa fiabilité. La mauvaise sensibilité et spécificité du test PCR pour $E$. coli ne permet pas son application en clinique et la recherche devra s'orienter vers des nouvelles cibles.

\section{FM I/14 Geburtshilfe \\ Identification du signal FSH inter-cycle par la mesure de la FSH urinaire (FSHU)}

Schwarz C., Bischof P., Jauch Klein T., Righetti A., Fournet Irion N., de Ziegler $D$.

Médecine de la Reproduction, Département de GynécologieObstétrique, Hôpitaux Universitaires de Genève

Introduction: Le signal FSH inter-cycle initie le recrutement folliculaire qui marque le début du cycle menstruel. Il survient généralement au 3e jour du cycle mais peut varier d'un cycle à l'autre et son amplitude est faible. Sa mesure dans le sérum (FSHs) requiert donc des prises de sang quotidiennes. Nous avons cherché à déterminer si le dosage de la FSH dans l'urine (FSHu) pouvait être une alternative non-invasive à la FSHs, en utilisant un modèle où la survenue du signal FSH inter-cycle est contrôlée par une prise ponctuelle d'E2. Méthode: Etude prospective chez 21 femmes infertiles ayant reçu de l'E2 ( $2 \mathrm{mg}$ per os, $2 \times /$ jour ou $0.1 \mathrm{mg} /$ jour transdermique) du 25e jour du cycle précédent au 1er Vendredi après les règles. Les échantillons de sang et de la 1ère urine du matin ont été récoltés pendant 7 jours, à partir du dernier jour sous E2 (valeur de base), pour évaluer la FSH (+ créatinine dans l'urine). Les mesures de FSHu ont été faites après filtration, en utilisant la même méthodologie que pour le sérum. Les résultats sont exprimés en $\mathrm{mIU} / \mathrm{ml}$ pour la FSHs et la FSHu et en $\mathrm{mIU} / \mathrm{mmol}$ de créatinine pour la $\mathrm{FSHu} / \mathrm{Cr}$. Une échographie a été effectuée 12 jours après l'arrêt de l'E2 pour la recherche de follicules en croissance $(>12 \mathrm{~mm})$. Résultats: $\mathrm{La} \mathrm{FSHu}$ et $\mathrm{FSHu} / \mathrm{Cr}$ montrent un bonne corrélation avec la FSHs ( $r=0,52$ et $0,63, p<0,0001$ et $p<0,0001$ pour FSHu et $\mathrm{FSHu} / \mathrm{Cr}$, respectivement). Chez 15/21 patientes qui ont eu un pic de FSHs, celui-ci a été confirmé par un pic de FSHu survenant 2-4
Abstracts, gynécologie suisse, Lugano, 2005 
jours après l'arrêt de l'E2. Chez ces femmes, l'évaluation échographique à $\mathrm{J} 12$ a montré une ovulation imminente. Chez 12 de ces 15 femmes, l'acmé du pic inter-cycle de la FSHu survint le même jour que celui de la FSHs et 1 jour plus tard, chez les 3 autres. L'amplitude du signal FSHu est en moyenne 3,2 fois plus élevée que celle de la FSHs. Chez 6/21 femmes, un pic FSH inter-cycle n'a pas été décelé ni par la FSHs ni par la FSHu et il n'y a pas eu d'ovulation. Conclusion: Nos résultats indiquent que le signal FSH intercycle peut être identifié et son amplitude mesurée par le dosage de la FSH urinaire. Cette approche permet des évaluations plus fines de la physiologie ovarienne que celles fournies par les dosages sanguins.

\section{FM I/15 Geburtshilfe \\ La loi relative à la recherche sur les cellules souches n'est-elle pas déjà dépassée?}

Wirthner D., Megalo A., Senn A., Urner F., Chanson A., Primi M.-P., Germond M.

Unité de Médecine de la Reproduction, Département de Gynécologie-Obstétrique, Centre Hospitalier Universitaire Vaudois, Lausanne

Introduction: La loi fédérale relative à la recherche sur les cellules souches embryonnaires (LRE) a été largement acceptée par le peuple suisse le 28 novembre 2004 (66,4\% des votants et 100\% des cantons l'ont acceptée). La LRE autorise l'utilisation d'embryons surnuméraires pour la production de cellules souches. La loi sur la procréation médicalement assistée (LPMA) interdit la congélation d'embryons. La majorité des embryons surnuméraires ont été congelés avant 2001. La LPMA autorise la congélation des zygotes pour une période de 5 ans. Au-delà, les zygotes doivent être détruits et ne peuvent pas être utilisés pour la production de cellules souches. But: Faire prendre conscience du nombre élevé de zygotes congelés en Suisse et de l'absence d'alternative autre que leur destruction s'ils ne sont pas utilisés pour la procréation. Méthode: Etude rétrospective du nombre de zygotes et d'embryons congelés, transférés ou détruits du 1.1.1988-31.12.2004 dans notre unité. Résultats: De 1988 à 2004, 3396 ponctions d'ovocytes (OPU) ont été effectuées dans notre centre, 15769 zygotes et 2392 embryons ont été congelés et 11673 zygotes versus 2069 embryons ont été décongelés. Il reste 4096 zygotes versus 323 embryons cryoconservés à ce jour. Pour la période 2001-2004, 16 embryons versus 7879 zygotes ont été congelés. Selon les données FIVNAT-CH, pour l'année 2002: 5452 zygotes versus 96 embryons ont été cryoconservés en Suisse. Discussion: La LRE concerne essentiellement les embryons surnuméraires congelés avant 2001. En effet, le nombre d'embryons congelés depuis le premier janvier 2001 est très faible. Le nombre d'embryons potentiellement disponibles pour la recherche est très bas en comparaison du nombre de zygotes surnuméraires qui augmente annuellement. Aucune alternative n'est proposée pour les zygotes surnuméraires excepté leur destruction à 5 ans. La possibilité de réponse à la demande des couples qui souhaitent offrir leur zygotes à la recherche est très importante. Le département fédéral de l'intérieur devrait rapidement statuer sur le devenir des zygotes surnuméraires, le résultat des votations ayant largement démontré le soutien du peuple suisse pour la recherche sur les cellules souches.

\section{FM II/20 Gynäkologie Erfahrungen mit Botulinum-A Toxin (BTX-A)-Injektionen in den Detrusormuskel in 43 Fällen mit therapieresistenter hyperreaktiver Blase}

Werner M. ${ }^{\mathrm{a}}$, Schmid D.M. ${ }^{\mathrm{b}}$, Perucchini D. ${ }^{\mathrm{a}}$, Hauri D. ${ }^{\mathrm{b}}$, Fink D. ${ }^{\mathrm{a}}$

${ }^{a}$ Kinik für Gynäkologie und ${ }^{\text {b} K l i n i k ~ f u ̈ r ~ U r o l o g i e, ~}$

Universitätsspital Zürich

Einführung: Ziel dieser prospektiven Studie war die Untersuchung der Wirksamkeit von Botulinum-A Toxin (BTX-A)-Injektionen in den Detrusormuskel bei Patientinnen mit hyperreaktiver Blase (OAB), therapieresistent auf Anticholinergika und Physiotherapie. Material und Methoden: Bei 43 Patientinnen (Durchschnittsalter 62 J.; 24-89 J.) mit nicht-neurogener hyperreaktiver Blase und Dranginkontinenz trotz hoher Dosen Anticholinergika wurden unter zystoskopischer Kontrolle an 20 verschiedenen Stellen 100 Einheiten BTX-A in den Detrusormuskel injiziert. Vor der Behandlung wurden alle Patientinnen urodynamisch abgeklärt sowie Miktionsverhalten, neurologischer Status, Urinproben und Blasenbiopsien erhoben. Analysiert wurden Reflexvolumen (RV), maximale Blasenkapazität (MBC), Detrusorcompliance (DC) sowie Drangverhalten und Miktionsfrequenz/Nykturie. Klinische und urodynamische Kontrollen erfolgten 6-8 Wochen nach der Behandlung. Ergebnisse: Insgesamt zeigten $88 \%$ der Patientinnen eine signifikante Verbesserung ihrer Blasenfunktion bezüglich subjektiver Beschwerden und urodynamischer Daten: Drangbeschwerden verschwanden komplett in 76\% und Dranginkontinenz in $80 \%$ innerhalb 1-2 Wochen nach der BTX-A Injektion. Die Miktionsfrequenz sank von 15 auf 7 Miktionen/Tag und die Nykturie von 5 auf 2. Die MBC stieg im Mittel von 269 auf $430 \mathrm{ml}$ an, die DC von 28 auf $54 \mathrm{ml} / \mathrm{cmH}_{2} \mathrm{O}$ und die Detrusorinstabilität (RV im Mittel $150 \mathrm{ml}$ ) verschwand in $81 \%$ der Fälle. Erster und zweiter Harndrang stiegen im Mittel von 127 auf $249 \mathrm{ml}$ und von 238 auf 415 $\mathrm{ml}$ an. Ausser 3 Patientinnen mit vorübergehender Blasenentleerungsstörung wurden keine Komplikationen beobachtet. Nur in ein paar wenigen Patientinnen mit präoperativ geringer DC war der klinische Erfolg gering. Die mittlere Wirkungsdauer war $9( \pm 2)$ Monate. Schlussfolgerung: Unsere Ergebnisse zeigen, dass BTXA Injektionen in den Detrusormuskel eine wirksame und sichere Option in der Behandlungsstrategie der therapieresisteneten hyperreaktiven Blase darstellen.

\section{FM II/21 Gynäkologie \\ Traitement laparoscopique du prolapsus génital: résultats d'une série de 42 cas de suspension latérale avec prothèse}

Dubuisson J.B., Jacob S., Wenger J.M., Dubé M.J.

Département de Gynécologie et d'Obstétrique,

Hôpitaux Universitaires de Genève

Introduction: La suspension latérale avec prothèse par laparoscopie est une technique que nous faisons depuis 10 ans [J Gynecol Surg 1998;14:153-159]. Nous rapportons notre expérience genevoise de cette technique personnelle. Matériel et méthodes: Il s'agit d'une analyse prospective de cas consécutifs de prolapsus génital (associés ou non à une incontinence urinaire d'effort) traités par suspension latérale laparoscopique. Les études de suivi furent réalisées pour évaluer les résultats de la chirurgie. Quarante-deux patientes (âge moyen: 53,12 \pm 9,88 (34-76) ans, IMC: 26,75 \pm 4,54 (1837)) avec un prolapsus génital de Stade II and III selon la classifica- 
tion POPQ ont été traitées. Une cystocèle Stade II et III fut notée dans $88 \%$ des cas, une hysterocèle Stade II et III dans $40 \%$ des cas, une rectocèle de Stade II et III dans $48 \%$ des cas. Toutes les candidates à la laparoscopie ont été incluses. L'intervention a consisté en une double colporrhaphie antérieure et postérieure, un réattachement des ligaments utérosacrés au torus uterinum et en une suspension latérale (au-dessus des crêtes iliaques) en utilisant un filet de mersilène $(30 \times 3 \mathrm{~cm})$ selon la technique que nous avons déjà publiée. Un Burch fut réalisé en cas d'incontinence urinaire d'effort et une périnéorraphie en cas de délabrement périnéal. Résultats: La technique opératoire s'est déroulée avec succès dans les 42 cas sans conversion en laparotomie. Dans 52,38\% des cas, un Burch a été associé, dans $16,6 \%$ des cas une hystérectomie subtotale. Le temps opératoire a été de 191,95 \pm 46,43 (120-360) min. Seulement 1 complication opératoire $(2,38 \%)$ (plaie vésicale traitée par laparoscopie) et 2 complications postopératoires $(4,76 \%)$ ( 1 hématome du Retzius spontanément résolutif, 1 hyperalgie au point de fixation latérale aponévrotique de la prothèse traitée au 4ème jour par section du fil sous anesthésie locale) furent notées. Des 42 patientes opérées et suivies (3,02 $\pm 2,72$ (1-12 mois), 39 rapportent une disparition de leurs symptômes et une amélioration de leur qualité de vie. Un résultat anatomique excellent est noté chez 41 patientes $(97,6 \%)$. Conclusion: Cette série de 42 patientes confirme l'amélioration de leur qualité de vie après suspension latérale laparoscopique.

\section{FM II/22 Gynäkologie \\ Weshalb die korrekte suburethrale Lage des TVT-Bandes wichtig ist}

Scheiner D., Perucchini D., Fink D.

Klinik für Gynäkologie, Departement Frauenheilkunde, Universitätsspital Zürich

Einführung: Bei der TVT-Operation ('tension-free vaginal tape') wird die Stabilisierung der mittleren Urethra angestrebt. Das $10 \mathrm{~mm}$ breite Band soll weder eine Elevation noch Obstruktion bewirken, sondern als Matrix für eine Neufixation der Urethra durch Einsprossung von Fibroblasten dienen. Die korrekte midurethrale Platzierung des Bandes soll beachtet werden. Wir untersuchten den Einfluss der Bandlage auf die Kontinenz, Restharnmenge und Miktion. Methodik: Bei 49 Patientinnen wurden die prä- und postoperativen Befunde der standardisierten urodynamischen Untersuchung, bestehend aus einem Fragebogen, klinischem Untersuch, Urodynamik, Perinealsonographie und Miktiometrie, analysiert. Als Messpunkt zur Bestimmung der Bandlage in Bezug zur Urethralänge definierten wir die perinealsonographisch dargestellte Bandmitte suburethral im Sagitalschnitt, wobei 0\% dem Meatus internus und 100\% dem Meatus externus entsprach. Die postoperative Kontinenz, Restharnmenge und Miktion werteten wir in Abhängigkeit der Bandlage aus. Die nicht normal verteilten Daten analysierten wir mit dem Wilcoxon- resp. Mann-Whitney-, nominal skalierte mit dem $\chi^{2}$ - resp. Fisher's exakten Test. Ergebnisse: Das Durchschnittsalter lag bei 66,1 Jahren (SD 9,0; 43,8$79,7)$ und war bei hypotoner Urethra $(\mathrm{n}=21)$ mit 71,0 Jahren deutlich höher als bei normotoner Urethra $(\mathrm{n}=28)$ mit 62,5 Jahren $(p=0,00)$. Der Hustentest war postoperativ bei $89,3 \%$ der Frauen mit normotoner und 73,7\% mit hypotoner Urethra negativ (kontinent). 75,5\% der Bänder lagen korrekt unter dem mittleren Urethradrittel. Das Band (Bandmitte) lag durchschnittlich leicht distal der Urethramitte bei $55,2 \%$ (28,8 bis $82,6 \%)$ der Urethralänge. Kein Band lag am Blasenhals. Zwischen Bandlage und Kontinenz bestand kein Zusammenhang $(\mathrm{p}=0,12)$, während mit zunehmender Nähe des TVT zum Meatus internus die postoperative Restharnmenge signifikant zu- und die maximale Flussrate tendenziell abnahm. Bei einer Bandlage zwischen 58 und 100\% kam es nie zu Restharnmengen über $100 \mathrm{ml}(\mathrm{p}=0,01)$. Schlussfolgerung: Das TVT lag meist korrekt midurethral. Die Kontinenz wurde durch die Bandlage nicht beeinflusst, während die Restharnmenge bei Blasenhals-naher Lage zu- und die maximale Flussrate abnahm.

\section{FM II/23 Gynäkologie \\ Transurethrale Injektionen: Zuidex versus Contigen - Effektivität und Akzeptanz}

\author{
Stähler van Amerongen K., Kuhn A., Dreher E.
}

Universitäts-Frauenklinik, Inselspital Bern

Einleitung: Ziel ist die prospektive Evaluation der Behandlung einer Belastungsinkontinenz mit transurethraler Injektion mit Zuidex versus Contigen hinsichtlich Effektivität und Akzeptanz. Patientinnen und Methode: 15 Patientinnen wurden mit transurethralen Injektionen behandelt. Die Zuordnung der Injektionen mit Zuidex (Hyaluronsäurederivat) oder Contigen (Kollagen) erfolgte randomisiert. 8 Patientinnen sind mit Zuidex und 7 Patientinnen mit Contigen behandelt worden. Das Alter der Probandinnen betrug median 57 Jahre (range 34-93). Alle Patientinnen hatten vor der Operation einen positiven Hustentest. Analysiert wurden Husten-, PAD-Test, Restharnvolumen, Combur, Uroflow, Patientenzufriedenheit und durchgeführte Inkontinenzoperationen. Ergebnisse: Das Follow-up wurde im Medianen nach 12 Monaten (range 10-17) durchgeführt. In der Zuidexgruppe $\mathrm{n}=8$ hatten die Hälfte der Patientinnen eine TVT-Operation nach der Follow-up-Zeit erhalten. In der Contigengruppe dagegen ist bei allen 7 Patientinnen keine weitere Operation erfolgt. In der Kollagengruppe hatten $5 \mathrm{~Pa}-$ tientinnen einen pos. Hustentest und 4 einen pos. PAD-Test. In der Zuidexgruppe dagegen hatten 2 einen pos. Husten- und PAD-Test. Bei der Befragung der Zufriedenheit waren in der Zuidexgruppe 6 Patientinnen mit dem Operationsergebnis zufrieden und 2 gaben eine Verbesserung an. In der Contigengruppe waren 5 zufrieden und 2 gaben ebenfalls eine Verbesserung an. Bei allen Patientinnen waren Restharnvolumen und Urinstix unauffällig. In der Kollagengruppe hat sich der max. Flow signifikant von $38,6 \mathrm{ml}$ im Medianen (range 22-65) präoperativ auf 24,4 $\mathrm{ml}$ im Medianen (range 5,9$61,8)$ postoperativ verringert. Die Miktionsdauer hat sich von $34 \mathrm{~s}$ im Medianen (range 12-54) auf 25,6 s (range 6-48) verkürzt. In der Zuidexgruppe ist ebenfalls eine Verringerung des max. Flow von $27.5 \mathrm{ml}$ im Medianen (range 12-70) auf $20,9 \mathrm{ml}$ im Medianen (range 4,3-50,2) zu verzeichnen, aber die Miktionsdauer hat sich signifikant von 19,5 s im Medianen (range 10-31) auf 33,45 s im Medianen (range 11-102,5) verlängert. Schlussfolgerung: In der Zuidexgruppe haben wir eine Erfolgsrate von nur 50\%, was möglicherweise auf die unterschiedliche Applikationsart zurückzuführen ist. Die Kollagengruppe zeigt eine grössere Zufriedenheit, obwohl die Patientinnen nicht vollständig kontinent sind.

\section{FM II/24 Gynäkologie \\ Botulinumtoxin $A$ bei der Behandlung der nicht neurogenen Detrusorhyperaktivität: 9-Monatsergebnisse}

Kuschel S. ${ }^{\mathrm{b}}$, Werner M. ${ }^{\mathrm{a}}$, Schmid D. ${ }^{\mathrm{a}}$, Schüssler B. ${ }^{\mathrm{b}}$

${ }^{a}$ Frauenklinik, Universitätsspital Zürich,

${ }^{\text {b}}$ Neue Frauenklinik, Kantonsspital Luzern

Einleitung: Botulinumtoxin A (BTX-A) ist eine etablierte Therapie bei der neurogenen Detrusorhyperaktivität (DH). Im Rah- 
men einer prospektiven und urodynamisch (urodyn.) basierten Beobachtungsstudie konnten bei der nicht neurogenen DH vergleichbar gute Resultate bezogen auf die Zunahme der Blasenkapazität (MCBC), Abnahme der Inkontinenzepisoden und Steigerung der Lebensqualität (QoL) in der Kurzzeitanalyse erzielt werden. Ziel dieser Studie war es, erste längerfristige Daten des gleichen Kollektivs aufzuzeigen. Material und Methode: $26(n=26)$ Patientinnen mit Over-active-bladder wet (OAB/wet) und einer urodyn. nachgewiesenen DH sowie einer bisher erfolglosen Therapie mit Anticholinergika wurde im Rahmen einer prospektiven Beobachtungsstudie an 30 verschiedenen Stellen 100 Einheiten BTX-A in den Detrusor injiziert. Prä- und postop. sowie nach $4(\mathrm{n}=26), 12(\mathrm{n}=24)$ und 36 Wochen $(n=21)$ wurde eine urodyn. Messung durchgeführt und die QoL mit Hilfe des validierten deutschen King's Health Questionnaire erfasst. Ergebnisse: Die MCBC und die Compliance waren mit $326 \mathrm{ml}$ bzw. $34 \mathrm{ml} / \mathrm{cm} \mathrm{H}_{2} \mathrm{O}$ bei 36 Wochen gegenüber $216 \mathrm{ml} \mathrm{bzw.} 14 \mathrm{ml} / \mathrm{cm} \mathrm{H}_{2} \mathrm{O}$ präop. stat. sig. erhöht $(\mathrm{p}<0,0001$ bzw. $\mathrm{p}=0,012$ ). Zystometrisch gesicherte Detrusorkontraktionen (DK) fanden sich nach 36 Wochen bei 12/21 Patientinnen. Gegenüber der präop. Situation, in der alle (26/26) Patientinnen DK aufgewiesen hatten, war der Rückgang stat. sig. $(p=0,012)$, im Vergleich mit den Ergebnissen nach 12 Wochen aber deutlich ungünstiger (8/24). Die Tagesmiktionsfrequenz war ebenfalls gegenüber dem präop. Ausgangswert stat. sig. erniedrigt (8,7 vs. $11,7, \mathrm{p}<$ 0,0001). Im Vergleich zur präop. Situation zeigte sich bei der QoL immer noch eine stat. sig. Besserung z.B. bei in- und ausserhäuslichen Aktivitäten, der Möglichkeit zu verreisen, Effekte auf den Nachtschlaf und der Notwendigkeit, Vorlagen zu tragen (Summenscore von $10 \mathrm{KHQ}$-Inkontinenz-Fragen: $\mathrm{p}=<0,0001$ ), obwohl sich die urodyn. Parameter gegenüber 12 Wochen verschlechtert hatten. Schlussfolgerung: Auch 36 Wochen nach einer Botoxinjektion finden sich noch stat. sig. und urodyn. sowie auf die subjektive QoL bezogen relevante Verbesserungen gegenüber der Ausgangssituation, obwohl die Wirksamkeit erwartungsgemäss abgenommen hat.

\section{FM II/25 Gynäkologie \\ Endometriose im Septum rectovaginale: Meistens ist eine Darmresektion notwendig?}

Ochsenbein-Imhof N. ${ }^{\mathrm{a}}$, Gloor B. ${ }^{\mathrm{b}}$, Fleischmann A. ${ }^{\mathrm{c}}$, Dreher E. ${ }^{a}$, Mueller M.D. ${ }^{\text {a }}$

${ }^{a}$ Kliniken und Polikliniken für Frauenheilkunde, Inselspital Bern, ${ }^{b}$ Klinik für viszerale und Transplantationschirurgie, Inselspital Bern, 'Institut für Pathologie der Universität Bern

Einführung: Die korrekte chirurgische Therapie der Endometriose erfordert eine Exzision aller makroskopisch erkennbaren Herde. Bei der tief infiltrierenden Endometriose des Septum rectovaginale stellt sich oft die Frage, ob eine Darmresektion notwendig ist. Ziel der vorliegenden Arbeit war, die Häufigkeit einer Endometriose-Darmwandinfiltration im Darmresektat zu untersuchen und so festzustellen, ob die laparoskopische Beurteilung einer möglichen Darmwandinfiltration adäquat ist. Material und Methode: Retrospektive Analyse der Endometriose-Patientinnen, bei welchen zwischen Januar 2002 und Dezember 2004 eine Darmresektion wegen Verdacht auf Endometriose-Infiltration der Darmwand erfolgte. Alle Darmresektate wurden histologisch untersucht. Resultate: An der Universitäts-Frauenklinik Bern wurden, im angegebenen Zeitraum, 206 Laparoskopien wegen Endometriose durchgeführt. Bei 37 (18\%) Patientinnen wurde klinisch ein Endometrioseknoten im Septum rectovaginale diagnostiziert. Intraoperativ wurde in 19 dieser Fälle (51,4\%) eine Endometriose-Infiltration der Darmwand laparoskopisch vermutet, weshalb eine laparoskopische Darmresektion erfolgte. Histologisch konnte bei allen Präparaten $(100 \%)$ ein Endometriose-Befall der Darmwand (bis zur Muscularis propria) bestätigt werden. Schlussfolgerungen: Bei entsprechender Erfahrung ist die laparoskopische Beurteilung einer möglichen Infiltration der Darmwand adäquat. Eine ausgedehnte chirurgische Resektion ist gerechtfertigt, denn die Koagulation oder Laserevaporisation ist, bei einer Invasionstiefe bis in die Muscularis propria des Darmes, zur vollständigen Exzision der Endometrioseherde im Septum rectovaginale sicher nicht genügend.

\section{FM III/30 Gynäkologie \\ Outcome hinsichtlich Blasen-, Darm- und Sexualfunktion nach vorderer und hinterer Plastik: Eine Studie an 128 Patientinnen}

\author{
Kuhn A. ${ }^{\mathrm{a}}$, Dreher E. ${ }^{\mathrm{b}}$ \\ aPrincess Anne Hospital, Southampton, UK, \\ ${ }^{\mathrm{b}}$ Frauenklinik, Inselspital Bern
}

Einleitung: Im Rahmen einer prospektiven Studie untersuchen wir die Patientinnenakzeptanz und das Outcome hinsichtlich anatomischem Resultat, Blasen-, Darm- und Sexualfunktion sowie intra- und postoperative Komplikationen bei Zystozelen- und Rektozelenoperationen unter reiner Lokalanästhesie mit Adrenalinzusatz. Patientinnen und Methode: Zwischen November 2000 und Dezember 2001 haben wir 128 Patientinnen mit symptomatischen Zysto- oder Rektozelen oder einer Kombination von beidem unter Lokalanästhesie/Adrenalingemisch operiert. Prä- und postoperativ wurden Miktionsprobleme, Stuhlsymptomatik sowie Sexualfunktion mittels Quality of Life Questionnaire und Sheffield Prolapse Questionnaire erfasst und ein Prolapsstaging mittels ICS POP-Score durchgeführt. Resultate: Der Follow-up betrug im Median 32 Monate. Es wurden 68 vordere Plastiken, 52 hintere Plastiken und acht kombinierte vordere und hintere Plastiken in dieser Studie durchgeführt. Alle Patientinnen bis auf zwei würden bei einem erneuten Eingriff wieder die Lokalanästhesie wählen. Bei einem Verbrauch von 15-30 ml LA-Adrenalingemisch betrug der intraoperative Blutverlust 3-36 ml (Median $12 \mathrm{ml}$ ), die Operationsdauer 18-29 min (Median 23 min). Keine Patientin benötigte starke Schmerzmittel postoperativ. Bei zwei Patientinnen kam es zu Miktionsstörungen postoperativ nach vorderer Plastik, die eine Kathetereinlage über mehrere Tage erforderten. Die objektive Erfolgsrate betrug $88 \%$ für vordere Plastiken, $87 \%$ für hintere Plastiken und 63\% für kombinierte Eingriffe. Das Vorfallgefühl konnte bei der der vorderen Plastik hochsignifikant verbessert werden ( $p=0,0001)$ und bei der hinteren Plastik signifikant verbessert werden $(\mathrm{p}=0,0049)$. Zu keiner signifikanten Veränderung ist es bei der Obstipation gekommen, und Dyspareunie wurde signifikant $(\mathrm{p}=0,002)$ bei der hinteren Plastik, nicht aber bei der vorderen Kolporrhaphie oder den kombinierten Eingriffen verbessert. Diskussion: Die Durchführung von Prolapsoperationen unter Lokalanästhesie ist gut akzeptiert von Seiten der Patientin und zeigt postoperativ gute anatomische und funktionelle Ergebnisse. Langzeitresultate bleiben abzuwarten. 


\section{FM III/31 Gynäkologie \\ Häusliche Gewalt - Wahrnehmen - Intervenieren}

Bass $B .^{a}$, Weingartner $M .^{b}$, von Castelberg $B .^{a}$

${ }^{a}$ Frauenklinik Maternité, StadtspitalTriemli Zürich,

${ }^{b}$ Büro für Gleichstellung von Mann und Frau der Stadt Zürich

Einführung: Das Projekt «Häusliche Gewalt» (HG) entwickelt ein Modell, wie mit dem Thema HG in einer Klinik umgegangen werden soll. HG bezeichnet physische, psychische, sexuelle, soziale und ökonomische Gewalt unter erwachsenen Menschen, die in einer engen sozialen Beziehung stehen. Ziel des Projektes ist die Sensibilisierung der Klinikmitarbeitenden für HG sowie die Erarbeitung von Grundlagen und Instrumenten für den Umgang mit betroffenen Patientinnen. Das Projekt beinhaltet eine Studie, Personalschulung sowie die Entwicklung von Leitlinien. Wissenschaftliche Grundlagen: Im Frühjahr 2003 wurden die Mitarbeitenden der Maternité über ihre Wahrnehmung von HG bei Patientinnen und zu ihren bisherigen Handlungsmöglichkeiten befragt. $65 \%$ gaben an, dass sie in den letzten 3 Monaten zu mindestens einer von Gewalt betroffenen Patientin Kontakt hatten. Es folgte eine schriftliche Befragung von 1772 Patientinnen der Maternité. In der Befragung berichtet jede 10. Patientin über aktuelle Gewaltbetroffenheit. Jede 4. erlitt in ihrem Erwachsenenleben in stärkerem Ausmass Gewalt im sozialen Nahraum. HG kommt in allen soziokulturellen Milieus und Altersgruppen vor und hinterlässt körperliche, psychische und psychosomatische Spuren. Betroffene Frauen haben signifikant mehr gesundheitliche Beschwerden als Nichtbetroffene. Am ehesten suchen Gewaltbetroffene Unterstützung im medizinisch-psychologischen Bereich, wobei viele Frauen ohne professionelle Hilfe bleiben. Ein Screening zu Gewalterfahrungen findet eine sehr hohe Akzeptanz. [D. Gloor, H. Meier: Frauen, Gesundheit und Gewalt im sozialen Nahraum. 2004 Ed. soziothek Bern]. Folgerungen: Die Ergebnisse der Studie veranlassten die Erarbeitung von Leitlinien und die Einführung eines Screenings. Die Leitlinien sollen zu mehr Handlungssicherheit bei den Mitarbeitenden führen und ein einheitliches Vorgehen gewährleisten. Klinik-interne und -externe Fachpersonen erarbeiteten die Leitlinien und bereiteten die interne Schulung vor. In einem Basisseminar zur Vermittlung von Hintergrundwissen und in einem Interventionsseminar zur Vorbereitung der Einführung von Leitlinien und Screening am 1.3.2005 wurden alle Mitarbeitenden mit Patientinnenkontakt geschult. Nach einer Probephase von 6 Monaten wird das Projekt evaluiert und die Ergebnisse publiziert.

\section{FM III/32 Gynäkologie \\ Does Incontinence to Flatus Affect Quality of Life?}

Weil A., Otero M., Floris L., Sangalli M.R., Boulvain M., Irion O., Faltin D.L.

Département de Gynécologie-Obstétrique,

Hôpitaux Universitaires de Genève

Introduction: Skeptical colleagues have contested that flatal incontinence affect quality of life and suggest that it should not be perceived as a disease. Methods: As part of a study on the longterm effect of delivery on the pelvic floor, we evaluated the association between incontinence to flatus and quality of life with a specific instrument, the fecal incontinence quality of life score, a multi-dimensional score with four sub-scales (lifestyle, coping, depression, and embarrassment) and a generic instrument, the SF-12
Table 1. Flatus incontinence and quality of life

\begin{tabular}{lcccc}
\hline Incontinence to flatus & Never & $<1 \times /$ week & $1 \times /$ week & $\mathrm{p}$ \\
\hline Fecal incontinence QoL: & $\mathrm{n}=377$ & & $\mathrm{n}=111$ & $\mathrm{n}=27$ \\
Lifestyle & 3.9 & 3.8 & 3.4 & $<0.0001$ \\
Coping & 3.8 & 3.5 & 3.1 & $<0.0001$ \\
Depression & 3.5 & 3.4 & 3.0 & 0.008 \\
Embarrassment & 4.6 & 3.2 & 2.7 & $<0.0001$ \\
SF-12 mental health & 46.1 & 45.6 & 42.7 & $<0.07$ \\
SF-12 physical health & 47.4 & 46.8 & 46.9 & $<0.42$ \\
\hline
\end{tabular}

Physical and Mental health scales. We excluded women with incontinence to liquid or solid stools. Results: 472 women responded to the questionnaire and had no incontinence to solid or liquid stools. Their mean age was 47 years (range 36 to 61 ), 74\% were married, $77 \%$ worked, $80 \%$ were multiparous. Incontinence to flatus less than once a week was reported by $20 \%$ and once a week or more by 4\% (table 1). Conclusion: Incontinence to flatus affects all domains of quality of life. It is not associated with overall poor physical health, but should be considered as a disease worth diagnosis and treatment.

Fonds National Suisse de la Recherche Scientifique no 3255907.98 et 3200-064056.00.

\section{FM III/33 Gynäkologie \\ Das klimakterische Syndrom - Ein Vergleich zwischen Jung und Alt}

Bodmer C. ${ }^{\text {a }}$ Limoni C. ${ }^{\text {b }}$,Wunder D. ${ }^{a}$, Birkhäuser M. ${ }^{a}$

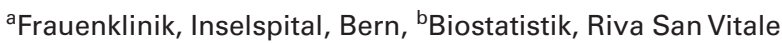

Einführung: Man geht davon aus, dass das klimakterische Syndrom ein typisches Erscheinungsbild der perimenopausalen Frau darstellt. Es kann mit der «Green Climacteric Scale» erfasst werden. Ziel dieser Arbeit war es, die Inzidenz dieses Syndroms bei einer Gruppe von jungen Frauen zu eruieren und gleichzeitig mit einer älteren perimenopausalen Population zu vergleichen. Material und Methoden: 900 junge Frauen aus verschiedenen Sprechstunden der Frauenklinik Bern (allgemeine Poliklinik, gynäkologische Endokrinologie und Familienplanung) sowie 760 ältere Frauen aus unserem Menopausen-Zentrum erhielten einen modifizierten «Greene Climacteric Scale»-Fragebogen nach Hause geschickt mit der Bitte, diesen auszufüllen und anonym an uns zurückzuschicken. Die Durchführung dieser Umfrage wurde von der Ethikkommission Bern bewilligt. Ergebnisse: 295 junge Frauen mit einem Durchschnittsalter von 28 Jahren sowie 339 perimenopausale Frauen mit einem Durchschnittsalter von 55 Jahren haben den Fragebogen ausgefüllt und anonym an uns zurückgeschickt. Folgende Symptome traten sowohl bei den jungen als auch bei den älteren Frauen mehr oder weniger ausgeprägt in gleichen Massen auf: Nervosität/Angespanntheit, Unausgeglichenheit/aufbrausend, Konzentrationsschwäche, müde/energielos, Desinteresse, unglückliches/depressives Gefühl, Schwindel/Ohnmachtsanfälle, Kopfschmerzen, Gefühl eines sinnlosen Lebens sowie verstärkte Neigung zum Grübeln. Weinanfälle und Reizbarkeit traten signifikant gehäuft bei den jungen Frauen auf. Die übrigen Symptome wie Wallungen, Schweissausbrüche, Palpitationen, Schlafstörungen, Panikattacken, Atemschwierigkeiten, Muskel- und Gelenkschmerzen, Libidoverlust, depressive Veränderungen, trockene Schleimhäute, Urgesymptome, taktile Gefühlsstörungen sowie Engegefühl traten signifikant mehr bei den älteren, nicht aber bei den jüngeren Frauen auf. Schluss- 
folgerung: Bereits in jungen Jahren können die sonst für die Perimenopause typischen Symptome auftreten. Somit beinhaltet die «Greene Climacteric Scale» möglicherweise Fragen, die gar nicht speziell der Menopause zugeordnet werden sollten.

\section{FM III/34 Gynäkologie \\ Petites doses d'hCG (mini-hCG) en fin d'hyperstimulation ovarienne contrôlée (HOC) modérée avec insémination intra-utérine (IIU)}

Ventura P., Fondop J.J., Romoscanu I., Ibecheole V., Stalberg A., de Ziegler D.

Médecine de la Reproduction, Département de GynécologieObstétrique, Hôpitaux Universitaires de Genève

Introduction: Dans le cycle menstruel, la fin de la phase folliculaire a lieu en dominance LH alors que toute l'HOC se fait en mode FSH dominant. Aujourd'hui, il a été démontré qu'il est possible de passer en mode LH dominant en fin d'HOC fortes, en remplaçant la FSH par des petites doses d'hCG (mini-hCG). Nous avons voulu savoir si cette option peut être également envisagée dans des HOC délibérément faible induite hors FIV pour minimiser le risque de grossesse multiple. Méthode: 26 cycles d'HOC-IUI sont analysés rétrospectivement chez 21 femmes infertiles. Les cycles sont synchronisés par la prise ponctuelle de la pilule contraceptive. L'HOC débute au 4e jour post pilule en utilisant de la FSH/ hMG soit, seule $(\mathrm{n}=10)$ soit, en association avec du citrate de clomiféne $(n=11)$ ou l'inhibiteur de l'aromatase $(n=5)$. Les cycles d'HOC sont évalués au 7e et 10e jour par échographie et dosages. 75 UI d'hCG (0,1 CC de Prégnyl ${ }^{\circledR} 1500 \mathrm{UI} / 2 \mathrm{ml}$ d'eau) sont administrés sous-cut. dès qu'un seul follicule atteint $13 \mathrm{~mm}$. L'induction de l'ovulation est réalisée par l'injection sous-cut. de 5000 UI d'hCG dès qu'un follicule atteint $17 \mathrm{~mm}$ entre le $10 \mathrm{e}$ et $13 \mathrm{e}$ jour d'HOC. On analyse le nombre de cycles annulés et les taux de grossesses simples et multiples. Résultats: 4 cycles ont été annulés $(15 \%), 3$ pour risque de grossesse multiple $(12 \%)$, et 1 pour une élévation prématurée de la $\mathrm{P} 4$., peut être en rapport avec le minihCG. On observe 6 grossesses (27\%/IIU) dont 2 (33\%) sont multiples (une gemellaire et une quadruple dans un syndrome de Kallmann qui n'avait que 2 foll. $>14 \mathrm{~mm}$ le jour du déclenchement. Conclusion: Les taux de grossesses élevés indiquent que l'administration de mini-hCG est possible dans les HOC délibérément modérées induites hors FIV comme cela est possible dans les HOC plus fortes. La grossesse quadruple (réduite à 2) observée en cas de syndrome de Kallmann était inévitable car seul 2 follicules étaient $>14 \mathrm{~mm}$ le jour du déclenchement. La sélectivité du mini-hCG qui ne stimule que les foll. $>13 \mathrm{~mm}$ devrait permettre de réduire les risques de grossesses multiples. Nos résultats ne confirment cependant pas cela, peut-être en raison d'une amélioration de la fécondité des cycles avec mini-hCG.

\section{FM III/35 Gynäkologie \\ TVT $^{\circledR} /$ SPARC ${ }^{\circledR} /$ TOT-Saphyre $^{\circledR}$ : Vergleich einer persönlichen longitudinalen Serie}

Dost $F^{\mathrm{a}}$, Kuschel S. ${ }^{\mathrm{a}}$, Werner M. ${ }^{\mathrm{b}}$, Schüssler B. ${ }^{\mathrm{a}}$

${ }^{a}$ Neue Frauenklinik, Kantonsspital Luzern, brauenklinik, Universitätsspital Zürich

Einführung: Tensionfree Vaginal Tapes (TVT) unterscheiden sich heute im operativen Grundkonzept in 3 Versionen: retropubisch, vaginal-abdominal $\left(\mathrm{TVT}^{\circledR}\right)$, retropubisch abdomino-vaginal
(SPARC ${ }^{\circledR}$ ) und transobturatorisch (TOT-Saphyre ${ }^{\circledR}$ ), das sich durch eine Kombination aus einem Polypropylenband und einer Silikonverankerung auszeichnet. Eine Beschreibung eines Vergleichs zwischen den 3 Methoden liegt in der Literatur bisher nicht vor. Ziel dieser Studie war es, erste Daten der TOT-Saphyre-Operationstechnik mit der TVT- und Sparc-Technik in Bezug auf Wirksamkeit, Sicherheit und Veränderungen der Lebensqualität $(\mathrm{QoL})$ zu vergleichen. Material und Methode: In dieser prospektiven longitudinalen Beobachtungsstudie wurden ohne vorgängige Lernkurve und Systematik sämtliche während des Versuchszeitraums operierte Patientinnen $(\mathrm{n}=156)$ mit einer urodynamisch gesicherten SIK \pm begleitender Over-active-bladder $(\mathrm{OAB})$ wet/dry \pm Inkontinenz-Voroperationen in die Studie aufgenommen. $\mathrm{n}=34$ erhielten ein TVT, $n=55$ ein Sparc und $n=67$ ein TOT. Zur Bewertung der QoL erhielten alle Patientinnen prä- und postoperativ den validierten deutschen King's Health Questionnaire (KHQ) und den Patient Global Impression of Severity/Improvement (PGI). Definition «Heilung»: Kein Urinabgang, keine OAB (wet/dry) sowie QoL: «vollkommen glücklich» oder «zufrieden». Definition «Besserung»: Urinverlust maximal in Tropfen, keine OAB, sowie Qol: «vollkommen glücklich» oder «zufrieden» oder «weitgehend zufrieden». Ergebnisse: Bei 5,9\% der TVT-, 5,5\% der Sparc- und 0\% der TOT-Patientinnen kam es zur intraoperativen Blasenperforation. Bezüglich «Heilung» und «Besserung» zeigte sich kein stat. signifikanter Unterschied zwischen den drei Gruppen (TVT 43, Sparc 60 und TOT 43\% bzw. 63, 68 und 62\%). 87, 96 und 93\% der TVT-, Sparc- und TOT-Patientinnen gaben eine subjektive Besserung der Blasenbeschwerden an. Postoperativ trat bei $0 \% / 4 \%$, $2 \% / 4 \%$ und $5 \% / 5 \%$ der Patientinnen der TVT/Sparc/TOT-Gruppen eine OABwet/dry auf. Schlussfolgerung: Erstmals konnte gezeigt werden, dass die TOT-Saphyre-Technik subjektiv und objektiv vergleichbare Ergebnisse wie die TVT- oder die Sparc-Technik zur operativen Therapie der SIK liefert, aber wie erwartet kein Risiko für eine Blasenperforation beeinhaltet.

\section{FM IV/40 Geburtshilfe \\ Qui a besoin d'une consultation de périnéologie post-partum?}

Faltin D.L., Boulvain M., Floris L.A., Weil A., Irion O.

Département de Gynécologie et d'Obstétrique, Hôpitaux Universitaires de Genève

Introduction: Les pathologies périnéales sont fréquentes dans le post-partum. Connaître les facteurs de risque de ces lésions permet de sélectionner les patientes pouvant potentiellement bénéficier d'une consultation spécialisée post-partum. Matériel et méthode: Nous avons réalisé le suivi d'une cohorte de 1220 primipares et évalué la sévérité des symptômes de pathologies périnéales (douleurs et incontinence) trois mois et un an post-partum par questionnaire. Seul les symptômes présent au moins une fois par semaine étaient considérés significatifs. Résultats: 1090/1220 (89\%) des participantes ont répondu au questionnaire un an post-partum. Les symptômes ont été souvent rapportés ( $35 \%$ au total): incontinence urinaire $9 \%$, incontinence aux gaz $6 \%$, incontinence aux selles solides $1 \%$, douleurs périnéales $8 \%$, dyspareunie $19 \%$. Les caractéristiques prédictives des variables associées aux symptômes sont présentées dans le tableau 1.

Parmi les femmes symptomatiques 3 mois post-partum, 45\% l'étaient encore un an post-partum. Parmi les femmes qui n'étaient pas symptomatiques 3 mois post-partum, 21\% ont développé par la suite des symptômes. Conclusion: Les symptômes périnéaux sont très fréquents post-partum. Une stratégie basée sur les facteurs 


\begin{tabular}{lcccl}
\hline & RR & $(95 \% \mathrm{CI})$ & $\begin{array}{l}\text { Sensibilité } \\
\%\end{array}$ & $\begin{array}{l}\text { Spécificité } \\
\%\end{array}$ \\
\hline Déchirure III/IV & 1,4 & $(1,1-1,7)$ & 10 & 94 \\
Acc instrumenté & 1,2 & $(1,0-1,4)$ & 44 & 62 \\
Poids $>4$ kg & 1,2 & $(0,9-1,6)$ & 8 & 94 \\
L'âge $>30$ ans & 1,2 & $(1,0-1,4)$ & 47 & 59 \\
Symptômes 3 mois pp & 2,2 & $(1,8-2,6)$ & 74 & 53 \\
\hline
\end{tabular}

de risque obstétricaux est inefficace pour sélectionner les patientes pouvant bénéficier d'un avis spécialisé. Le dépistage post-partum par l'anamnèse systématique constitue probablement la meilleure approche en gardant à l'esprit la fréquence élevée de symptômes se déclarant tardivement.

Fonds National Suisse de la Recherche Scientifique no 3255907.98 et 3200-064056.00.

\section{FM IV/41 Geburtshilfe \\ Computerbasierte Dokumentation in der Schwangerenvorsorge}

Kurmanavicius J., Burkhardt T., Zimmermann R.

Klinik für Geburtshilfe, Universitätsspital Zürich

Einführung: Entwicklung eines klinisch orientierten computerbasierten Informationssystems für die ambulante Schwangerenbetreuung sowie eines elektronischen Mutterpasses. Methoden: Objektorientierte Programmierung der Software mit Microsoft FoxPro 8.0 auf der Basis einer Microsoft SQL Server Datenbank. Ergebnisse: Es wurde ein strukturiertes, modular aufgebautes, speziell für die Bedürfnisse der Geburtshilfe zugeschnittenes Informationssystem programmiert. In der Hauptmaske der Schwangerschaftskontrolle werden der Verlauf von schwangerschaftsrelevanten klinischen Parameter, Laborparameter, eine Zusammenfassung der letzten Ultraschalluntersuchung, Medikamente sowie allgemein Status und Diagnosen angezeigt. Die Daten aller Schwangerschaftskontrollen können in Form eines zusammenfassenden Berichtes ausgedruckt werden und auch auf einen USB-MemoryStick, zusammen mit Ultraschallberichten, Ultraschallbildern etc. als elektronischer Mutterpass im PDF-Format, übertragen werden. Die Daten können jederzeit von der Schwangeren bzw. vom zuweisenden Arzt auf Windows- oder Mac-Computern eingesehen werden. Schlussfolgerung: Eine strukturierte Dateneingabe und der einfache Zugang zu relevanten Informationen erleichtert und verkürzt den Zeitaufwand für die Dokumentation. Ein computerbasiertes Klinikinformationssystem speziell für die Geburtshilfe erhöht die Qualität der medizinischen Versorgung und vermeidet in Kombination mit einem elektronischen Mutterpass unnötige Doppeluntersuchungen und Doppelerfassungen.

\section{FM IV/42 Geburtshilfe \\ Mechanismen des Fetal Programming: Das Vagus/Sympathicus-System}

Schäffer L. ${ }^{\mathrm{a}}$, Burkhardt T. ${ }^{\mathrm{a}}$, Arlettaz R. ${ }^{\mathrm{b}}$, Beinder E. $^{\mathrm{a}}$

${ }^{a}$ Klinik für Geburtshilfe, ${ }^{b}$ Klinik für Neonatologie,

Universitätsspital Zürich

Hintergrund: Eine intrauterine Mangelversorgung während der fetalen Entwicklung ist mit einem erhöhten Risiko für kardiovas- kuläre u. metabolische Erkrankungen im späteren Leben assoziiert. Ursache für diese Veränderungen ist möglicherweise eine Fehlprogrammierung verschiedener biologischer Regulationssysteme bereits im Mutterleib. Einer der möglichen Mechanismen dafür könnte eine erhöhte Aktivität des Sympathikussystems des Feten sein, welches postpartal persistieren und dadurch eine ungünstige Adaptation des kardiovaskulären Systems verursachen könnte. Wir analysierten daher das Sympathicus/Vagus-System bei wachstumsretardierten Kindern prä- und postpartal. Methoden: CTGs von Kindern mit einem Geburtsgewicht $<5$. Perzentile (SGA) und von normgewichtigen Kindern (AGA) wurden hinsichtlich Herzfrequenz und Frequenzvariabilität analysiert. Postpartal wurden am 3. Lebenstag durchgeführte $24 \mathrm{~h}$ Holter EKGs im Hinblick auf verschiedene Parameter für Herzfrequenzvariabilität als Indikator für eine Sympathikus-Aktivierung analysiert. Ergebnisse: 20 SGAFeten und 20 AGA-Feten wurden untersucht. Die pränatale CTGAnalyse ergab eine mittlere HF von 140 bpm sowohl in SGA- wie auch in AGA-Feten. Die fetale Herzfrequenzvariabilität lag bei 14.6 bei SGA- und 16.8 Schlägen bei AGA-Feten ohne einen signifikanten Unterschied. Mittels $24 \mathrm{~h}$ EKG postpartal erhobene geometrische und time-domain-Indexe für Herzfrequenzvariabilität zeigten keine signifikanten Veränderungen bei SGA-Feten im Vergleich zu AGA-Feten. Schlussfolgerung: Die Herzfrequenzvariabilität als Zeichen einer sympathischen Aktivierung zeigt keine Veränderungen in SGA-Neugeborenen. Die Adaptation des Feten an eine intrauterine Mangelversorgung führt demnach nicht zu einem postnatalen Ungleichgewicht des Sympathicus/Vagus-Systems.

\section{FM IV/43 Geburtshilfe \\ Riech- und Schmeckvermögen in der Schwangerschaft und post partum}

Ochsenbein-Kölble N. ${ }^{a}$, von Mering R. $^{a}$, Zimmermann R. $^{a}$, Hummel . $^{\mathrm{b}}$

${ }^{a}$ Klinik für Geburtshilfe, Universitätsspital Zürich,

${ }^{b}$ Hals-Nasen-Ohren-Klinik, Universität Dresden, Deutschland

Einführung: Viele Schwangere berichten in der Schwangerschaft von einer veränderten Geruchs- u. Geschmackswahrnehmung. Inwieweit dies von der individuellen olfaktorischen u. gustatorischen Sensibilität abhängig ist, ist nach wie vor unklar. Studienziel war es daher, die Frage zu beantworten, ob sich die olfaktorische bzw. gustatorische Sensibilität im Verlauf der Schwangerschaft ändert. Methodik: 38 schwangere u. 46 nicht-schwangere Frauen wurden zu 4 Messzeitpunkten (MP) untersucht. Bei Schwangeren lag MP A zw. der 5. und 14. Schwangerschaftswoche (SSW), MP B zw. der 15. und 24. SSW, MP C zw. der 35. und 41. SSW u. MP D 6 Wo. post partum. Kontrollpersonen wurden im Abstand von 8-12 Wochen untersucht. Die olfaktorische Sensibilität wurde anhand eines validierten Riechtests, den sog. «Sniffin-Sticks», erfasst. Zusätzlich bewerteten die Teilnehmerinnen Intensität und Hedonik von «natürlichen» Riechproben. Die Schmeckschwelle wurde für alle 4 Geschmacksrichtungen (süss, sauer, salzig, bitter) bei MP A-D untersucht. Die Intensität u. Hedonik der Geschmacksrichtungen wurden erfasst. Ergebnisse: Bei MP A zeigten sich keine signifikanten Unterschiede hinsichtlich der Riechfähigkeit zw. den beiden Gruppen. Über den gesamten Messzeitraums hinweg kam es zu einer signifikanten Abnahme des Riechvermögens der Schwangeren, was v.a. auf Änderungen der Riechschwellen zurückgeführt werden konnte, die post partum noch immer erniedrigt waren. Diese «objektiven» Befunde standen den «subjektiven» gegenüber, wobei sich Schwangere als riechempfindlicher einstuften als Nicht-schwangere. Auf hedonischer Ebene beurteilten beispiels- 
weise schwangere Frauen den Geruch von Essig bei MP B u. C als signifikant angenehmer als die nicht-schwangeren Frauen. Schwangere Frauen zeigten eine erniedrigte Schmeckfähigkeit, vor allem für «bitter», bewerteten ihr Schmeckvermögen jedoch höher als das der nicht-schwangeren. Schlussfolgerung: Während der Schwangerschaft finden sich Veränderungen der olfaktorischen u. der gustatorischen Sensitivität, wobei es eine Diskrepanz zwischen «objektiven» und «subjektiven» Befunden gibt. Eine Erklärung hierfür finden sich möglicherweise in der schwangerschaftsinduzierten Änderung der kognitiven Verarbeitung überschwellig dargebotener chemosensorischer Information.

\section{FM IV/44 Geburtshilfe \\ Blutflussgeschwindigkeit in der Vene von Nabelschnüren mit singulärer Arterie}

Hoffmann K., Lanz S., Raio L.

Universitätsfrauenklinik, Inselspital Bern

Einführung: In einer vorausgegangenen Studie konnte gezeigt werden, dass bei Feten mit singulären Nabelschnurarterien (SNA) die mittlere Blutflussgeschwindigkeit während eines Herzzyklus (TAMx) im Ductus venosus (DV) in über 50\% der Fälle unterhalb der 5. Perzentile lag. Es wurde postuliert, dass neben einer möglicherweise von der Norm abweichenden Anatomie des Ductus venosus auch der Nabelring oder die Morphologie der Nabelschnur (grosse Vene und Arterie, in der Regel weniger Windungen) diesbezüglich eine Rolle spielen könnte. Die vorliegende Arbeit hat zum Ziel, die TAMx in der Vene in Fällen mit SNA zu untersuchen. Material und Methoden: Bei Fällen mit SNA wurde bei jeder Konsultation die Blutflussgeschwindigkeit in der Nabelschnurvene gemessen (TAMx). Die Messungen erfolgten bei fetaler Ruhe und fehlenden Atembewegungen an einem frei flottierenden Abschnitt der Nabelschnur. Das Dopplergate wurde dem Gefässlumen angepasst, der Insonationswinkel wurde in jedem Fall korrigiert und lag immer unter 30. Die ermittelten Werte wurden mit publizierten Normkurven verglichen. Ergebnisse: Im Zeitraum von 1,5 Jahren wurden insgesamt 27 Fälle mit SNA untersucht. In diesem Kollektiv erfolgten 66 TAMx-Messungen. In 46 von 66 Fällen $(69,7 \%)$ lag die TAMx unterhalb der 50. Perzentile für das jeweilige Gestationsalter, in $24,2 \%(16 / 66)$ unterhalb der 5. Perzentile. Schlussfolgerung: Ähnlich wie bei den Flussgeschwindigkeiten im DV von Feten mit SNA ist auch in der Nabelschnurvene in einem relativ hohen Anteil der Fälle eine auffällig tiefe venöse Geschwindigkeit zu verzeichnen. Dies könnte teilweise die niedrigen Flussgeschwindigkeiten im DV erklären.

\section{FM IV/45 Geburtshilfe \\ Das feto-fetale Transfusionssyndrom: Ergebnisse nach Laserbehandlung, ein Update}

Neukomm C.B., Kuhn P., Dürig P., Schneider H., Raio L.

Perinatalzentrum, Universitätsfrauenklinik, Inselspital Bern

Einführung: Das feto-fetale Transfusionssyndrom (FFTS) stellt eine schwerwiegende Komplikation bei monochorialen Zwillingen dar. Unbehandelt beträgt die Letalität bis zu 90\%. Verursacht wird das FFTS durch plazentare Anastomosen. Die Therapie der Wahl ist die Laserkoagulation dieser kommunizierenden Gefässe. Im Folgenden berichten wir über die Resultate der in Bern und Schaffhausen (Kuhn P.) durchgeführten Lasereingriffe. Material und Me- thoden: Die Daten und Ergebnisse aus beiden Gruppen wurden gepoolt und gemeinsam betrachtet. Die angewendete Technik der fetoskopischen Laserkoagulation war in beiden Kliniken die gleiche. Ergebnisse: Es wurden 34 Zwillingsschwangerschaften mit FFTS in die Studie aufgenommen. Das mediane Gestationsalter betrug bei Lasertherapie 20,5 Wochen $(16,1-24,4)$, bei Geburt (Abort) 32,7 Wochen (16,2-39,2). Lediglich 5/27 (18,5\%) der Schwangerschaften dauerten länger als 37 Wochen. Pränatal überlebten in 18/34 (52,9\%) Fällen beide Kinder, in 9/34 (26,5\%) Fällen nur ein Kind (in 8 Fällen ist der Donor verstorben). In 27/34 (79,4\%) Fällen überlebte also mindestens ein Kind, und von insgesamt $68 \mathrm{Kin}-$ dern somit 45 (66,2\%) den Eingriff. Postnatal sind 3 weitere Kinder an frühgeburtlichen Komplikationen verstorben. Bei einem Kind musste wegen einer trockenen Gangrän ein Unterschenkel amputiert werden. Dies war die einzige mögliche eingriffsassoziierte Komplikation in unserem Kollektiv. Mütterlicherseits wurden alle Eingriffe gut toleriert. Im Verlauf haben zwei Frauen eine schwere Präeklampsie entwickelt, eine mit HELLP-Syndrom, die andere mit Eklampsie. Schlussfolgerung: Unsere Resultate sind vergleichbar mit denjenigen der Literatur. Die perinatale Mortalität kann durch den Eingriff gesenkt werden. Ein ungelöstes Problem ist jedoch die hohe Frühgeburtlichkeit in diesem Kollektiv, welche die Morbidität negativ beeinflusst. Diese Studie wird zur Untersuchung der längerfristigen Ergebnisse noch weitergeführt.

\section{FM V/50 Pränatale Medizin \\ Fetch-Studie: Prospektive fetale \\ Kardiologie-Studie in der Schweiz}

Fasnacht M. ${ }^{\mathrm{a}}$, Pfammatter J.P. ${ }^{\mathrm{b}}$, Ghisla R. ${ }^{\mathrm{c}}$, Sekarski N. ${ }^{\mathrm{d}}$ Steinmann $H_{.}{ }^{\mathrm{e}}$, Kuen $P^{\mathrm{f}}$, Günthard J. ${ }^{\mathrm{g}}$

Kinderkardiologie, Universitätskinderklinik a Zürich, ${ }^{\mathrm{b}}$ Bern, dLausanne, ' ${ }^{\mathrm{d} B a s e l}$, Kinderspital ' $\mathrm{C}$ St. Gallen, ${ }^{\mathrm{e}}$ Aarau, 'Luzern

Einführung: In der Schweiz gibt es 70-75 000 Lebendgeburten pro Jahr. Bei einer Inzidenz von angeborenen Herzfehlern von $0.7-$ $0.8 \%$ würden jährlich 490-600 betroffene Neugeborene erwartet, davon 230-260 mit hämodynamisch signifikanten und pränatal diagnostizierbaren Herzfehlern. Angeborene Herzfehler sollten mittels Ultraschallscreening durch Geburtshelfer entdeckt und in ein tertiäres fetales Kardiologie-Zentrum zur Diagnosebestätigung und weiteren Therapie überwiesen werden. Ziel dieser prospektiven Studie war es, die pränatal diagnostizierten Herzfehler zu analysieren und mit den postnatal diagnostizierten zu vergleichen. Methode: Prospektive Studie aller fetalen Echokardiographien in 7 tertiären fetalen Kardiologie-Zentren während einer Zeitdauer von 2 Jahren (1.7.01-30.6.03) und prospektives Erfassen aller Kinder mit hämodynamisch relevantem und intrauterin diagnostizierbarem Herzfehler geboren während derselben Zeitperiode. Resultate: Insgesamt wurden 433 fetale Untersuchungen durchgeführt, davon 35\% vor der 24 SSW. 272 (62\%) waren normal, 94 (22\%) zeigten einen schweren Herzfehler, 21 (5\%) einen leichten Herzfehler und 46 (11\%) Arrhythmien. Die Sensitivität der fetal gestellten Diagnosen war 99\%, die Spezifität 87\%. Postnatal wurden 306 Kinder mit schwerem angeborenem Herzfehler diagnostiziert. Das heisst, 23\% aller 400 angeborenen schweren Herzfehler wurden pränatal diagnostiziert. Das Spektrum der fetal diagnostizierten Herzfehler war: single-ventricle-Malformationen 22 (23\%), conotruncale Malformationen 30 (33\%), Septumdefekte 28 (30\%), Klappenanomalien 7 (7\%), andere 7 (7\%). Bezugnehmend auf die fetale und postnatale Gruppe wurden 50\% single-ventricle-Malformationen, 22\% conotruncale Malformationen, 17\% Septumdefekte, $12 \%$ Klappenanomalien und 20\% andere Malformationen in- 
trauterin diagnostiziert. Schlussfolgerungen: Obwohl nicht nur angeborene Herzfehler, welche im 4-Kammerblick diagnostizierbar sind, pränatal entdeckt wurden, wurden lediglich 23\% aller intrauterin diagnostizierbaren angeborenen Herzfehler pränatal entdeckt. Dies bedeutet, dass mehr Gewicht auf das pränatale Screening gelegt werden sollte.

\section{FM V/51 Pränatale Medizin Outcome fetal versus postnatal diagnostizierter angeborener Herzfehler: Schweizer Multizenter-Studie}

Günthard J. ${ }^{\mathrm{a}}$, Pfammatter J.P. ${ }^{\mathrm{b}}$, Ghisla R. ${ }^{\mathrm{c}}$, Sekarski N. ${ }^{\mathrm{d}}$, Steinmann $\mathrm{H}^{\mathrm{e}}$, Kuen $\mathrm{P}^{\mathrm{f}}$, Fasnacht $\mathrm{M}^{\mathrm{g}}$

Kinderkardiologie, Universitätskinderklinik ${ }^{a}$ Basel, bBern, dLausanne, ${ }^{\mathrm{g}}$ Zürich, Kinderspital ${ }^{\mathrm{c}}$ St. Gallen, ${ }^{\mathrm{e}}$ Aarau, ${ }^{\mathrm{f}}$ Luzern

Einführung: Bei 70-75 000 Lebendgeburten pro Jahr und einer Inzidenz angeborener Herzfehler (CHD) von $0.7-0.8 \%$ würden in der Schweiz jährlich 490-600 betroffene Neugeborene erwartet, davon 230-260 mit hämodynamisch signifikanten und pränatal diagnostizierbaren CHD. Ziel dieser prospektiven Studie war, das Outcome pränatal diagnostizierter CHD zu analysieren und mit den postnatal diagnostizierten zu vergleichen. Methode: Prospektive Studie aller fetalen Untersuchungen in 7 tertiären fetalen Kardiologie-Zentren während 2 Jahren (1.7.01-30.6.03) und prospektives Erfassen aller Kinder mit hämodynamisch relevantem und intrauterin diagnostizierbarem CHD geboren während derselben Zeitperiode. Resultate: Bei 94 Feten wurde ein schwerer CHD entdeckt. $22(23 \%)$ hatten eine single-ventricle, 30 (33\%) conotruncale, 7 (7\%) valvuläre, 28 (30\%) Septumdefekte und 7 (7\%) andere Malformationen. Das Gestationsalter bei Diagnosestellung war bei $32<24$ SSW, bei $54>24$ SSW und bei 8 nicht bekannt. In $70(74,5 \%)$ Fällen wünschten die Eltern eine postpartale Therapie, in $22(21 \%)$ keine Therapie. Zum intrauterinen Fruchttod kam es in 2 Fällen. In der Nichtbehandlungsgruppe wurde ein Schwangerschaftsabbruch bei 14/22, eine Comfort care postpartal bei $8 / 22$ gewählt. In der Behandlungsgruppe überlebten 52/70 >1 Jahr. 18/70 (10 ohne, 8 mit Behandlung) überlebten nicht. Gesamt überlebten 55,3\%. Bei 306 Kindern wurde der CHD postpartal entdeckt. Davon waren 21 (7\%) single-ventricle, 93 (31\%) conotruncale, 39 (13\%) valvuläre, $120(40 \%)$ Septumdefekte und $26(9 \%)$ andere Malformationen. $266(87 \%)$ überlebten >1 Jahr, 40 (13\%) starben, 19 ohne Intervention, 10 postoperativ und $10 \mathrm{CHD}$ unabhängig. Schlussfolgerungen: Die meisten Eltern von Kindern mit pränatal diagnostiziertem Herzfehler wünschten eine Behandlung. Allerdings könnte dies durch eine späte pränatale Diagnose gebiased sein. Das Outcome pränatal diagnostizierter CHD ist schlechter als das postnatal diagnostizierter. Grund dafür könnte der höhere Prozentsatz schwerer CHD (single-ventricle) in der pränatalen Gruppe sein.

\section{FM V/52 Pränatale Medizin \\ Können postnatal erhobene \\ Gewichtsstandards zur Diagnostik einer IUGR bei Frühgeborenen herangezogen werden?}

Burkhardt T., Zimmermann R., Kurmanavicius J.

Klinik für Geburtshilfe, Universitätsspital Zürich

Zielsetzung: Vergleich einer neu erstellten intrauterinen Gewichtsnormkurve mit Standardkurven von Neugeborenen. Material und Methode: Durchführung einer Cross-sectional-Studie zur
Berechnung von Normkurven für ultrasonographische Schätzgewichte bei Einlingsschwangerschaften. Es wurde jeweils das Schätzgewicht der ersten Ultraschalluntersuchung nach 20 SSW verwendet. In allen Fällen war das Gestationsalter durch einen Frühultraschall gesichert. Mittels Regressionsanalyse wurden intrauterine Normgewichtskurven berechnet. Diese Kurven wurden mit den Normkurven von Neugeborenengewichten verglichen. Ergebnisse: Die Schätzgewichte von 15724 Feten konnten ausgewertet werden. Zwischen 37 und $41 \mathrm{SSW}$ entprechen die intrauterinen Gewichtskurven denen von Neugeborengewichten. Zwischen 27 und 35 SSW liegt die 10. Perzentile der ultrasonographischen Schätzgewichte bis zu $300 \mathrm{~g}$ über der von Neugeborenengewichten (Winterthur-Kurve, Deutsche Kurve). Schlussfolgerung: Frühgeburten eignen sich nicht zur Erstellung von Gewichtsnormkurven, da sie im Vergleich zu gleichaltrigen intrauterin verbleibenden Kindern in einem hohen Mass eine Retardierung aufweisen.

\section{FM V/53 Pränatale Medizin \\ Evolution des D-dimères durant \\ I'accouchement et le post-partum}

Epiney M. ${ }^{\mathrm{a}}$, Boehlen $F^{\mathrm{b}}$, Boulvain M. ${ }^{\mathrm{a}}$, Reber $\mathrm{G}^{\mathrm{b}}$, Antonelli E. ${ }^{\mathrm{a}}$, Morales M. ${ }^{\mathrm{a}}$, de Moerloose P. ${ }^{\mathrm{b}}$, Irion O. ${ }^{\mathrm{a}}$

aDépartement de Gynécologie et d'Obstétrique,

bervice d'Angiologie et d'Hémostase, Hôpitaux Universitaires de Genève

Introduction: Dans la population générale le dosage plasmatique des D-dimères (DD) est une aide au diagnostic en cas de suspicion de maladie thromboembolique veineuse (MTEV) si $<500 \mathrm{ng} / \mathrm{ml}$. Pendant la grossesse l'élévation des DD les rend moins utiles et on ne connaît pas leur évolution dans le post-partum. L'objectif était de déterminer la cinétique des DD dans le post-partum et de définir à partir de quel moment leur utilisation est de nouveau possible dans l'exclusion de la MTEV. Matériel et méthode: Nous avons inclus 150 patientes accouchant à terme soit par césarienne $(n=50)$ soit par voie basse $(n=100)$ après une grossesse sans complication. Les DD ont été mesurés (test VIDAS D-dimer-New; (BioMérieux, France) à terme, dans les $2 \mathrm{~h}$ après l'accouchement (J0), puis à J1, J3, J10, J30 et J45. Résultats: En valeurs médianes (5ème et 95 ème percentile), les $\mathrm{DD}$ en $\mathrm{ng} / \mathrm{ml}$ sont pour les femmes avec accouchement par voie basse de 1405 (705-2578) à terme, 3989 (1694-8717) à J0, 2262 (1061-5859) à J1, 1262 (747-2303) à J3, 1284 (561-3117) à J10, 336 (155-931) à J30 et $236(127-555)$ à J45; pour les césariennes de 1544 (735-2455), 3432 (116710168), 2439 (1133-7178), 1542 (678-2602), 2061 (1028-3863), $359(208-794)$ et $279(159-636)$ respectivement. Les taux de DD sont tous supérieurs à $500 \mathrm{ng} / \mathrm{ml}$ à terme, à $\mathrm{J} 0, \mathrm{~J} 1$ et $\mathrm{J} 3$ et sont significativement plus élevés après césarienne et accouchement instrumenté qu'après AVB spontané. Ils diminuent rapidement dans les 3 premiers jours post-partum mais à J10 on observe une augmentation. A J30 et J45, respectivement 79 et $93 \%$ des femmes du groupe accouchement par voie basse et 70 et $83 \%$ des patientes avec césarienne ont des taux $<500 \mathrm{ng} / \mathrm{ml}$. Les saignements, l'allaitement ou la prophylaxie par héparine de bas poids moléculaire ne modifient pas significativement les DD. Conclusion: Notre étude fournit des intervalles de référence pour le post-partum et indique que les DD n'ont qu'une utilité limitée pour exclure une MTEV dans les 4 semaines après un accouchement.Une anticoagulation prophylactique est souvent recommandée après césarienne.L'extension de cette pratique après un accouchement instrumenté pourrait être discutée.
Abstracts, gynécologie suisse, Lugano, 2005 


\section{FM V/54 Pränatale Medizin}

Améliorer la sécurité en salle d'accouchement en approfondissant la coopération

\section{inter-métiers: Une expérience aux HUG}

Morales M.A. ${ }^{a}$, Kloetzer L. ${ }^{\mathrm{b}}$, Pfister R. ${ }^{\mathrm{c}}$, Billieux M.H. ${ }^{\mathrm{a}}$, Kern Ch. ${ }^{\mathrm{d}}$, Pariès $\mathrm{J}^{\mathrm{b}}$, Irion $O{ }^{\text {a }}$

${ }^{a}$ Service d'Obstétrique, ${ }^{b}$ Dédale, ${ }^{c}$ Service de Néonatologie,

dService d'Anesthésie, Hôpitaux Universitaires de Genève

Introduction: En salle d'accouchement, la coopération entre les métiers est particulièrement importante. Sept métiers au moins sont amenés à y intervenir quotidiennement, simultanément ou successivement, souvent dans l'urgence. Notre objectif est d'améliorer la sécurité en salle d'accouchement en approfondissant la coopération inter-métiers. Matériel et méthodes: Ce travail cherche à rendre compte d'une expérience d'apprentissage organisationnel centré sur la sécurité en salle d'accouchement. De janvier à décembre 2004, 220 professionnels de la salle d'accouchement, de tous les métiers concernés, ont ainsi participé à une vingtaine de séminaires «Ensemble» animés par leurs collègues, professionnels de la salle d'accouchement engagés dans le projet. Notre approche s'attache à solliciter l'expérience et le vécu de chacun à travers des débats organisés entre collaborateurs et à récolter les remontées pour les transmettre aux différents départements concernés. Résultats: Après 20 séminaires, 96 remontées différentes ont été dénombrées. A ce jour, 14 de ces remontées ont été réglées, le plus souvent par de petits groupes de travail. Par nature, les problèmes qui remontent sont complexes: s'ils remontent, c'est souvent que les équipes n'ont pas réussi à les résoudre par ellesmêmes. Ces problèmes se trouvent aux frontières de ce que l'organisation de l'hôpital et les collectifs eux-mêmes ont déjà su mettre en place. Conclusion: En conclusion, nous voudrions souligner que cette démarche, exigeante en matière d'implication des personnes à tous les niveaux de l'organisation, ne va pas de soi mais qu'elle crée des espaces d'échanges très productifs, en particulier pour les professionnels isolés dans leurs logiques de métier, et entre les professionnels de terrain et leurs cadres, dans des organisations sous tension. Dans une activité d'urgence fortement coopérative, il existe des marges de progrès importantes hors de la compétence individuelle. Les indicateurs de ces progrès sont difficiles à mettre en place.

\section{FM V/55 Pränatale Medizin \\ Selective Feticide in Complicated Monochorionic Twin Pregnancies Using Ultrasound-Guided Bipolar Cord Coagulation. Experience with 46 Cases}

Robyr R. ${ }^{\mathrm{a}, \mathrm{b}}$, Yamamoto M. ${ }^{\mathrm{a}}$, Ville $Y^{\mathrm{a}}$

aDépartement de Gynécologie et d'Obstétrique, Centre Hospitalier Intercommunal, Poissy-St-Germain, France, bDépartement de Gynécologie et d'Obstétrique, Hôpitaux Universitaires de Genève

Objective: The purpose of this study was to review our experience with selective feticide in the management of complicated monochorionic twin pregnancies, using ultrasound-guided cord coagulation with a bipolar forceps. Methods: Retrospective analysis. All umbilical cord coagulations performed at our institution in the second trimester of pregnancy between 1999 and 2003 were included. Ultrasound-guided coagulation of the umbilical cord was performed with a $2.5-\mathrm{mm}$ bipolar forceps under local anesthesia.
Cord coagulation was offered in order to improve the outcome of the co-twin. Indications of the procedure, pregnancy complications and pediatric follow-up were reviewed. Results: 46 patients with monochorionic twin pregnancies underwent this procedure. Our indications were twin reverse arterial perfusion sequence $(n=17)$, severe malformation in one twin $(n=7)$, agonal presentation of one twin in twin to twin transfusion syndrome (TTTS) $(n=22)$. The procedure resulted in six IUFD (13\%) and four neonatal deaths related to prematurity following preterm rupture of the membranes. Rupture of membranes before 34 weeks occurred in $21 \%$ of the cases. One death occurred at 10 months of age because of bronchopulmonary dysplasia in a case of preterm delivery at 26 weeks. Technical failure occurred in 2 cases which led to IUFD of the survivor. IUFD was more likely to occur when the procedure was indicated before 18 weeks. Pediatric follow-up showed that all infants discharged alive but none were neurologically normal at 3 to 42 months. Overall survival rate with normal development was $69 \%$. Conclusions: This technique is effective in complicated monochorionic twin pregnancies, when the natural history is likely to severely affect the development of the normal co-twin. Prematurity remains a significant complication of the procedure.

\section{FM VI/60 Gynäkologie \\ Expression de la cycline B1 dans les lésions intraépithéliales et les cancers vulvaires: perspective d'un marqueur}

Beffa V. ${ }^{\mathrm{a}}$, Pelte M.F. ${ }^{\mathrm{b}}$, Bischof P. ${ }^{\mathrm{a}}$, Vlastos A.T. ${ }^{\mathrm{a}}$

${ }^{a}$ Département de Gynécologie et d'Obstétrique, ${ }^{\text {b Département }}$ de Pathologie, Hôpitaux Universitaires de Genève

But: La cycline B1 est une molécule clé dans la transition G2/M au cours du cycle cellulaire. L'augmentation de son expression est connue dans différentes tumeurs et dans les lésions intraépithéliales cervicales. Son expression dans les VIN et cancers de la vulve ainsi que son intêret clinique n'ont pas été étudiés. Méthode: Nous avons utilisé l'immunohistochimie pour examiner l'expression de la cycline B1 dans 41 specimens avec des lésions histologiques de type VIN1 (11), VIN2 (6), VIN3 (6), carcinome (10) et des epithélium normaux (8). Résultats: L'expression de la cycline B1 est observée plus fréquemment dans les VIN3 et cancers que VIN1, VIN2 ou tissu contrôle (valeur de $\mathrm{p}<0,0001$ dans tous les cas). Le pourcentage médian de cellules exprimant la cycline B1 va de 4 à $10,7 \%$ pour l'epithelium normal jusqu'au VIN2 (non statistiquement significatif) alors qu'il est de $22 \%$ pour les VIN3 et $20.7 \%$ pour les cancers $(p=0.59)$. Conclusion: L'expression de cycline B1 est clairement augmentée dans les VIN3 et cancers. Ces résultats sont similaires à ceux obtenus sur les CIN.

\section{FM VI/61 Gynäkologie \\ Role of Tumor Suppressor BARD1 in Malignant Transformation; Implication for Therapeutic Interventions}

\author{
Feki A. ${ }^{a}$, Faltin D. ${ }^{a}$, Vlastos A.-T. ${ }^{\mathrm{a}}$, Vlastos G. ${ }^{\mathrm{a}}$, Irion $O{ }^{\mathrm{a}}{ }^{\text {, }}$ \\ Irminger-Finger I. $^{\mathrm{b}}$ \\ aDepartment of Gynecology and Obstetrics, \\ biology of Aging Laboratory, Department of Geriatrics, \\ University Hospital of Geneva
}

The BRCA1-associated protein BARD1 is regarded as tumor suppressor in conjunction with BRCA1. We have shown that re- 
pression of BARD1 leads to pre-malignant transformation and genomic instability [Irminger-Finger et al., 1998]. However, endogenous or exogenous increase of BARD1 induces apoptosis in vivo and in vitro, while BARD1-repressed cells are resistant to apoptosis induced by hypoxia or genotoxic drugs [Irminger-Finger et al., 2001]. The protein sequence required for apoptosis function of BARD1 coincides with tumor-associated missense mutations, thus confirming BARD1's role in tumor suppression through induction of apoptosis [Jefford et al., 2004]. Increased BARD1 expression might play a role in tissue homeostasis as observed in testis, spleen, or colon. Consistently, BARD1 expression is lost in some malignant tumors. However, in tumors expressing full length BARD1, it is cleaved during apoptosis, as it is in vitro, and the p67 cleavage product acts as immunogenic anti-tumorigenic agent in a rat animal model of colon cancer [Gautier et al. 2000; Jefford et al., 2004].

To test the immunogenic and anti-tumorigenic activity of BARD1 we identified a rat ovarian cancer cell line, NuTu-19, which is deficient of BARD1, defective in apoptosis induction and upregulation of $\mathrm{p} 53$ protein levels in response to doxorubicin-induced genotoxic stress, in spite of a wild-type p53. NuTu-19 cells, when injected intraperitoneally, induce tumors and metastasis leading to death of the animal 7 weeks after injection. We used this animal model to test the efficiency of virus-delivered BARD1 in tumor cell killing in vitro and in vivo by transduction of NuTu-19 cells with inducible BARD1. Induction of BARD1 either caused cell death or rendered cells hyper-sensitive to apoptosis-inducing agents, in vitro. To evaluate the effect of BARD1 expression on tumor cell proliferation in vivo, fluorescence-tagged NuTu-GFP cells were generated. Tumor formation was reduced or abrogated in animals injected with viable BARD1-expressing NuTu-19 cells. Tumor growth is slowed down by injection of retroviral BARD1 into subcutaneous tumors. Immunohistochemistry, performed on tumor samples, demonstrates increased immune response in tumors after lentiviral BARD1 injection. We conclude that BARD1 plays a role in tumorigenesis both as apoptosis inducer and as immunogenic agent.

\section{FM VI/62 Gynäkologie \\ Endometriumkarzinom: Maximal therapeutischer Ansatz trotz minimal-invasiver Chirurgie}

Santi A. ${ }^{\mathrm{a}}$, GyrTh. ${ }^{\mathrm{b}}$, Eberhard M. ${ }^{\mathrm{c}}$, Lanz S. ${ }^{\mathrm{a}}$, Dreher E. ${ }^{\mathrm{a}}$, Mueller M.D. ${ }^{\mathrm{a}}$

${ }^{\mathrm{a}}$ Frauenklinik Inselspital Bern, ${ }^{\mathrm{b}}$ Ospedale Regionale Lugano, ${ }^{c}$ Kantonsspital Schaffhausen

Einführung: Die chirurgische Therapie ist ein wichtiger Teil der Behandlung des Endometriumkarzinoms. Adipositas und höheres Alter sind vielfach begleitende Nebendiagnosen, weshalb ein minimal-invasives Verfahren in diesem Kollektiv sinnvoll erscheint. Ziel der vorliegenden Studie war es, die Durchführbarkeit und den Nutzen der laparoskopischen Chirurgie in der Behandlung des Endometriumkarzinoms zu evaluieren. Patientinnen und Methoden: Retrospektive Analyse der Patientinnen mit Endometriumkarzinom, bei welchen von 1996 bis 2005 in Bern, Lugano und Schaffhausen eine totale laparoskopische Hysterektomie mit Adnexektomie beidseits und, wenn notwendig, eine laparoskopische pelvine und allenfalls paraaortale Lymphonodektomie durchgeführt wurden. Die Nachkontrollen erfolgten alle 3-6 Monate. Resultate: Einhundertdrei Patientinnen konnten analysiert werden. Das durchschnittliche Alter der Patientinnen lag bei 65.1 (47-88) Jahren. In der definitiven Diagnose lag bei 87 Patientinnen (84\%) ein FIGO Stadium I, bei 8 (8\%) ein Stadium II, bei $6(6 \%)$ ein Stadium III und bei 2 (2\%) ein Stadium IV vor. Die mittlere Operationszeit betrug $160 \mathrm{~min}(60-310 \mathrm{~min})$, der mittlere Blutverlust 230 (10-2000) ml. In 94 (91\%) Fällen wurde eine laparoskopische Lymphonodektomie vorgenommen. Sechs Fälle mussten in eine Laparotomie konvertiert werden. Die Anzahl der entfernten Lymphknoten lag bei 22,7 (2-42). In 7 (7\%) wurden positive Lymphknoten gefunden. Insgesamt kam es in $4(4 \%)$ zu postoperativen Komplikationen. Die Hospitalisationszeit lag bei 5,7 (2-13) Tagen. Nach einer mittleren Beobachtungszeit von 2,6 Jahren waren 98 (95\%) Patientinnen ohne Zeichen eines Rezidivs, 2 (2\%) sind am Tumor verstorben und in 3 (3\%) Fällen kam es zu einem lokalen bzw. intraabdominalen Rezidiv. In unserem Kollektiv sind keine PortsiteMetastasen aufgetreten. Schlussfolgerungen: Das Endometriumkarzinom kann, bei entsprechender Erfahrung, laparoskopisch operiert werden. Operationszeit sowie Anzahl der entfernten Lymphknoten sind vergleichbar mit den Ergebnissen der Laparotomie. Aufgrund fehlender Langzeitresultate sollte die laparoskopische Behandlung des Endometriumkarzinoms nur im Rahmen von Studien durchgeführt werden.

\section{FM VI/63 Gynäkologie \\ Ultraschallgestützte und stereotaktisch gesteuerte invasive Mammadiagnostik an der Univeritätsfrauenklinik Basel}

Schuller C., Zanetti R., Holzgreve W., Wight E.

Universitätsfrauenklinik Basel

Einführung: Die Früherkennung eines Mammakarzinoms und die Abklärung von Läsionen der Mamma sind heute dringende Anliegen seitens unserer Patientinnen. An unserer Klinik werden jährlich bis zu 200 palpable und nicht palpable Läsionen in der Mamma mittels sonographisch und stereotaktisch gesteuerter minimal invasiver Biopsiemethoden histologisch abgeklärt. Material und Methoden: Von 6/2002 bis 12/2003 wurden an unserer Klinik insgesamt 267 diagnostische Mammabiopsien durchgeführt. Davon waren 252 sonographisch und 15 stereotaktisch gesteuert. Die FNP kam in der Regel nur bei zystischen Befunden zum Einsatz. Indikationen für eine invasive Diagnostik waren Befunde der BIRADS Kategorie 3, 4 und 5 in der Mammasonographie und/oder Mammographie. Entsprechend dem histologischen Resultat wurde eine Operation durchgeführt oder die Läsion mittels Bildgebung weiterverfolgt (6-18 Monate). Ergebnisse: Im Anschluss an die insgesamt 267 Biopsien führten wir 38 Operationen durch (14,2\%), wobei sich 30 Karzinome fanden (CR 11,2\%). In 8 Fällen wurde trotz benigner Histologie eine Tumorektomie durchgeführt, $5 \times$ auf Wunsch der Patientin, $2 \times$ bei V.a. Phylloidestumor, $1 \times$ bei Diskrepanz zwischen Sonographie und Stanzbiopsie. Bei den 252 sonographisch gesteuerten Punktionen führten 33 zu einer Operation mit 26 bestätigten Karzinomen (CR 78\%). In 219 Fällen (82\%) konnte eine offene Biopsie zur Abklärung des Befundes vermieden werden. Bei den stereotaktischen Biopsien fand sich $1 \times$ LCIS und $4 \times$ eine maligne Histologie: $3 \times$ ein DCIS und $1 \times$ ein invasives Karzinom plus DCIS. Die postoperative definitive Histologie bestätigte die Diagnose in allen Fällen (CR 26\%). Auch hier liess sich in 11 Fällen eine offene Biopsie vermeiden (74\%). Schlussfolgerungen: Basierend auf unseren Ergebnissen und der Literatur sind die sonographisch und stereotaktisch gesteuerten Interventionen ein zuverlässiges Mittel, um die Dignität eines Befundes festzustellen. Diese Methoden können gewährleisten, bei abklärungsbedürftigen Befunden der Brust wenig invasiv zu bleiben, unnötige Ope-
Abstracts, gynécologie suisse, Lugano, 2005 
rationen zu vermeiden und Kosten zu sparen. Sie sind darüberhinaus bei Malignomen ein unverzichtbares Mittel zur optimalen Operationsplanung.

\section{FM VI/64 Gynäkologie \\ Pilotstudie: Verändert die Hysteroskopie das Tumorstadium des Endometrium-Karzinoms?}

Günter D. ${ }^{\mathrm{a}}$, Dreher E. ${ }^{\mathrm{a}}$, Mueller M.D. ${ }^{\mathrm{a}}$, Stucki D. ${ }^{\mathrm{b}}$, Lüscher K. $^{\mathrm{b}}$, Maurer $F^{\mathrm{b}}$

a Universitäts-Frauenklinik, Inselspital Bern,

${ }^{\mathrm{b}}$ Arbeitsgemeinschaft für Endoskopische Gynäkologie

Einführung: Postmenopausale (PMPB) bzw. perimenopausale atypische Blutungen werden meist mittels Hysteroskopie (HSC) und Curettage (CUR) abgeklärt. Ob es bei der HSC zur Verschleppung von malignen Zellen ins Abdomen kommt und ob dies prognostisch relevant ist, wird kontrovers diskutiert. Des weiteren existieren keine Studien, welche die bei HSC gebräuchlichen Spüllösungen bezüglich ihrer möglichen Tumorzelldissemination vergleichen. Material und Methode: Seit 02/04 werden, in einer multizentrisch angelegten Studie, Frauen mit PMPB vor der HSC erfasst und bzgl. der bei der HSC zu verwendenden Spüllösung (Ringer, $\mathrm{NaCl}$, Purisole, Glycocol) randomisiert. Wird die HSC von einer Staging Operation gefolgt, so werden die intraoperativ gewonnenen abdominalen Spülzytologien entsprechend den verwendeten Spüllösungen verglichen. Die abdominalen Spülzytologien nach HSC werden ebenfalls mit jenen von Patientinnen verglichen, bei welchen präoperativ nur eine Pipelle oder eine CUR durchgeführt wurde. Ergebnisse: Bis anhin wurden 51 Frauen rekrutiert. In 27 Fällen wurde ein Endometrium-Ca diagnostiziert. Präoperativ hatten 15 Patientinnen eine HSC \& CUR, 7 nur eine CUR und in 2 Fällen wurde die Diagnose mittels Pipelle gestellt. Zwei weitere wurden zufällig im Rahmen einer Hysterektomie, eines im Rahmen eines Stagings bei unklarem Tumor in abdomine gefunden. Drei Patientinnen wurden, bei zu grossem Operationsrisiko, nur mittels Radiotherapie behandelt. Bei 23 Patientinnen wurde eine Staging Operation durchgeführt (1 Patientin war in einer inkurablen Situation). Die Tumorstadien waren: FIGO IA: 1, IB: 9; IC: 6; II: 3; III: 4; Carcinosarkom: 1; nicht genauer definiert: 3. Schlussfolgerungen: Entsprechend den bisherigen Ergebnissen werden in jeder Gruppe 200 Patientinnen notwendig sein - ein Total von 2000 (mit Drop-out-Rate 40\%), um signifikante Unterschiede errechnen zu können. Diese Zahlen können nur durch eine gesamtschweizerische Studie erreicht werden.

\section{FM VI/65 Gynäkologie \\ Qualitätssicherung der Indikationsstellung und der Ergebnisse in der Gynäkologie am Beispiel der Hysterektomie}

Schilling J. ${ }^{a}$, Huth S. ${ }^{a}$, Hagmann D. ${ }^{a}$, Hess $T^{a}$,

Fink D. ${ }^{\mathrm{a}}$, Infanger . $^{\mathrm{a}}$, Wyss $P^{\mathrm{a}}$, Surbek $D$. $^{\mathrm{b}}$

aMitglieder der wissenschaftlichen Begleitgruppe, ${ }^{b}$ Präsident der Kommission Qualitätssicherung der gynécologie suisse

Ausgangslage: Die transparente Qualitätssicherung der Indikationsstellung und der Ergebnisse hat in der medizinischen Versorgung innerhalb der vergangenen zehn Jahre an Bedeutung gewonnen. Durch den Vergleich der Anwendung der Indikations- empfehlungen mit den tatsächlichen klinischen Ergebnissen können einerseits Systemaussagen gemacht werden, anderseits können Leitlinien überprüft und wenn angezeigt angepasst werden. Ziele: Prospektive kontinuierliche Erfassung der Angemessenheit, der Notwendigkeit und der Ergebnisse zur Tracerprozedur Hysterektomie, Validierung der von der SGGG akzeptierten Konsensleitlinien zur Indikationsstellung durch Ergebnisdaten und Evaluation der Machbarkeit der Übersetzung der Leitlinien in eine für die Bevölkerung verständliche Sprache sowie die Möglichkeit zur Integration der Datenerfassung in Klinikprofile. Methoden: Die Datenerfassung der Ergebnisse (physischer und psychosozialer Status unter Einbezug der Lebensqualität der Patientin) erfolgte jeweils bei Eintritt in die Spitäler (Baseline) und 16 Wochen nach erfolgter Operation. Die Daten der Adäquanz erfolgte präoperativ. Einschlusskriterien waren Patientinnen, welche zu einer elektiven vaginalen, laparoskopischen oder abdominalen Hysterektomie überwiesen wurden. Notfall- und karzinombedingte Hysterektomien wurden ausgeschlossen. Zur Beurteilung der Veränderung der funktionellen und psychosozialen Situation der Patientin wurde, basierend auf dem SF36, ein gewichteter Score gebildet. Resultate: Im Zeitraum vom 1. März bis zum 30. November 2004 konnten 153 Fälle analysiert werden: Für die Angemessenheitsauswertung ergaben sich folgende Ergebnisse: 51\% $(\mathrm{n}=56)$ der Fälle wurden gemäss den Konsensleitlinien der SGGG als angemessen eingestuft, $43 \%(n=37)$ nach Präferenz der Patientin und 15\% ( $=16)$ als nicht angemessen. Notwendigkeit gemäss Definition bestand in $58 \%(\mathrm{n}=56)$. Der Vergleich bezüglich Status (physisch und psychisch) zeigt 16 Wochen postoperativ für alle Patientinnen einen signifikanten Unterschied zur Baseline. Patientinnen, welche gemäss den Konsensleitlinien der SGGG zu den Kategorien «Angemessene Indikationsstellung» und der Gruppe «Präferenz der Patientin» zugeordnet wurden, zeigten betreffend Ergebnis einen um 30 Punkte besseren Scorewert im Vergleich zur Baseline $(p=0,02)$. Für Patientinnen ohne angemessene Indikationsstellung gemäss den Konsensleitlinien der SGGG konnte keine signifikante Verbesserung nachgewiesen werden $(p=0,6)$. Die Integration des Erfassungsinstruments in dasjenige der Arbeitsgemeinschaft Schweizerischer Frauenkliniken (ASF) zeigte sich abgesehen von der Patientenbefragungen als möglich. Die Machbarkeit der Übersetzung der Leitlinien zur Indikationsstellung der Gebärmutterentfernung in eine für die Bevölkerung verständliche Sprache zeigte sich als gegeben. Diskussion: Die Angemessenheits- und Notwendigkeitsraten dürfen im internationalen Vergleich als adäquate Nutzung medizinischer Möglichkeiten gewertet werden. Aufgrund der noch geringen Anzahl Datensätze einerseits innerhalb der verschiedenen Unterkapitel wie auch gesamthaft können noch keine validen Schlüsse gezogen werden. Die Frage, ob Patientinnen mit angemessenen Indikationsstellungen auch bessere Ergebnisse zeigen, darf trendmässig positiv beantwortet werden. Dies spricht für die Validität der Konsensleitlinien der SGGG. 
Poster

\section{P I/10 Psychosomatik \\ Prévention de l'accouchement avant terme par une prise en charge psychologique}

Qayoom Z. ${ }^{\mathrm{a}}$, Boulvain M. ${ }^{\mathrm{b}}$, Magnenat D. ${ }^{\mathrm{a}}$, Palacio-Espasa F. Irion $\mathrm{O}^{\mathrm{b}}$

aService de Psychiatrie de l'Enfant et de l'Adolescent,

${ }^{b}$ Département de Gynécologie et d'Obstétrique,

Hôpitaux Universitaires de Genève

L'accouchement prématuré est la première cause de mortalité et de morbidité périnatale. Malgré les nombreux traitements proposés, le risque d'accouchement avant terme concerne 10\% des grossesses et ne diminue pas. Différentes causes et facteurs de risque ont été mis en évidence, dont les facteurs psychosociaux. L'activité et le milieu professionnel, le stress lié aux antécédents de grossesses difficiles, les relations intergénérationelles conflictuelles et la difficulté à se représenter dans un rôle de mère pourraient expliquer le travail prématuré dans certains cas. Une étude [Mamelle et al.] a évalué l'effet d'une intervention psychologique chez des mères présentant une menace d'accouchement prématuré. Le premier groupe (309 femmes), pris en charge avant la mise en place du programme de soutien psychothérapeutique, constituait le groupe témoin. Le groupe expérimental (323 femmes) a bénéficié de la prise en charge psychologique en plus du traitement habituel. Les résultats ont montré que les femmes avec traitement psychologique présentaient un risque de prématurité de $12 \%$, par rapport à $26 \%$ dans le groupe témoin (RR: 0,5; IC 95\%: 0,3-0,7; $\mathrm{p}<0,001$ ). Même si ces résultats sont très prometteurs, on ne peut exclure un biais dû à des différences entre les femmes traitées avant et après le début de l'intervention. L'objectif de notre étude est de confirmer ces résultats dans le cadre d'un essai clinique randomisé. Les femmes admises pour menace d'accouchement prématuré avant 34 semaines sont randomisées à l'un des deux groupes suivants: prise en charge psychologique en plus du traitement habituel, ou traitement habituel seul. Les entretiens visent à identifier les facteurs psychologiques et les antécédents de grossesses compliquées pouvant être à l'origine de la menace d'accouchement prématuré. Le but des entretiens est de diminuer l'impact des facteurs de risque identifiés, ainsi que le stress lié à situation. Le protocole détaillé de l'essai, ainsi que des cas cliniques illustrant l'intervention pratiquée seront présentés. Si elle est démontrée efficace, cette prise en charge pourrait diminuer le risque d'accouchement prématuré et ses conséquences néonatales.

\section{P I/11 Psychosomatik \\ Sexual Dysfunction and Body Mass Index}

Bianchi-Demicheli F., Faltin D.L., Rollini C., Otero M., Floris L., Boulvain M., Irion $O$.

Départements de Gynécologie-Obstétrique et de Psychiatrie, Hôpitaux Universitaires de Genève

Introduction: The extent of the effect of excess weight on sexual function in women is not well known. Material and Method: As part of a study on the long-term effect of delivery on the pelvic floor, we evaluated the association between Body Mass Index and sexual function with the use of the female sexual function index
Table 1

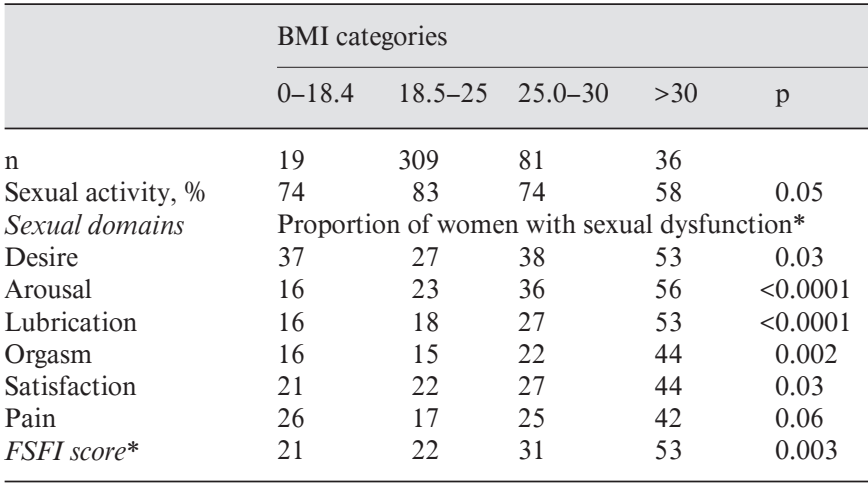

* Proportion of women under the 25th percentile.

(FSFI), a muldimensional scale summarizing desire, arousal, lubrication, orgasm, satisfaction, and pain in a global score. We defined sexual dysfunction as a score below the 25 th percentile of the scores distribution. Results: 445 women responded to the questionnaire. The BMI distribution according to WHO criteria was: $69 \%$ normal (BMI 18,5-25), 4\% underweight (BMI <18,5), 18\% mild overweight (BMI 25-30), 8\% overweight (BMI $>30)$. Fewer women with higher BMI had sexual activity and sexual dysfunction was more frequently reported by women with higher BMI: Table 1. Conclusion: Sexual function decreases with greater BMI. The association is stronger in the domains arousal and lubrication.

Fonds National Suisse de la Recherche Scientifique no 3255907.98 et 3200-064056.00.

\section{P I/12 Psychosomatik}

\section{Alles im Griff durch Bekenntnis zum Qualitätsmanagement}

Reize Ch. ${ }^{\mathrm{a}}$, Stierli $\mathrm{M}^{\mathrm{b}}{ }^{ }$, Beck $\mathrm{G}^{\mathrm{b}}$

aStabsstelle Chefärztekonferenz, Kantonsspital Olten,

${ }^{\mathrm{b}}$ Frauenklinik, Kantonsspital Olten

Einführung: Die Frauenklinik des Kantonsspitals Olten hat im Rahmen der kantonalen gesetzlichen Vorgaben innerhalb von 18 Monaten ein prozessorientiertes Qualitätsmanagementsystem (QMS) nach der International Standard Organisation (ISO) 9001:2000 aufgebaut. Das Zertifizierungaudit ist für März 2005 geplant. Material und Methoden: Die Frauenklinik wurde von der Spitalleitung beauftragt, ein QMS zu implementieren. Das daraufhin vom Kader der Frauenklinik in Zusammenarbeit mit der Stabsstelle Chefärztkonferenz definierte Projektziel war, ein funktionierendes QMS mit ISO-Zertifizierung aufzubauen, das als Qualitätsverbesserungsinstrument Eingang in die klinische Tätigkeit findet. Folgende Phasen wurden durchlaufen: (1) Einführungsschulung, (2) Definition des Vorgehens, Projektplanung, (3) Entwicklung eines Klinikleitbildes und einer Zielhierarchie, (4) Erstellen von: Handbuch, Haupt- und Unterstützungsprozesse, Arbeitsanweisungen, (5) Definition der relevanten medizinischen und administrativen Kennzahlen und Messgrössen, (6) Schulung aller Mitarbeiter, (7) Überprüfung des Projektstandes durch eine externe Vorauditierung, (8) Zertifizierungsaudit. Ergebnisse: Bereits während der Einführungsphase und der Etablierung von klar definierten Arbeitsgruppen und Ausschüssen verbesserte sich die Kommunikation zwischen den einzelnen Individuen und Berufsgruppen markant. Es entstanden für den klinischen Ablauf wichtige Arbeitsdo- 
kumente, die von jedem Arbeitsort elektronisch abrufbar sind. Schnittstellen zu anderen Kliniken wurden klar definiert, wo notwendig gemeinsame Dokumente erstellt. Ein Kennzahlensystem inkl. benchmarkfähigen medizinischen Messgrössen ermöglicht, dass das QMS als zentrales auf die Bedürfnisse der Klinik zugeschnittenes Führungsinstrument wertvolle Dienste leistet. Schlussfolgerung: Aufgrund obiger Erfahrungen können wir die Implementierung eines prozessorientierten QMS ohne Vorbehalte weiterempfehlen. Dadurch kann die Klinik auf ein Ziel ausgerichtet werden und eine kontinuierliche Verbesserung der klinischen Ergebnisse kann entstehen. Dieses System wird dem medizinisch akademischen Denkmuster gerecht. Eine Unterstützung während der Projektphase, die sich sowohl in der klinischen wie auch in der Qualitätswelt bewegen kann, beeinflusst den Projekterfolg entscheidend.

\section{P l/13 Psychosomatik \\ Functional Neuroimaging and Sexual Desire in Women: Where Are We 40 Years after the First Sexual Revolution?}

Ortigue S. ${ }^{\mathrm{a}}$, Bianchi-Demicheli $F^{\mathrm{b}}$

aDepartment of Neurology, University Hospital and Department of Fundamental Neurosciences, University Medical School, Geneva, 'bepartment of Obstetrics and Gynecology and Department of Psychiatry, University Hospital of Geneva

Since the first sexual revolution of the 1960s, brought about by the womens' rights movement and oral contraception, emancipation became a reality, and women entered into a period of free adult sexual equality that feminists fought for. Unfortunately, although young, women may suffer from sexual dysfunction (at least $43 \%$ of women). Low libido, or hypoactive sexual desire disorder is the most prevalent type of sexual dysfunction $(\sim 30 \%)$. It is marked by a consistent absence of sexual fantasies, a loss of desire, difficulty in achieving orgasm, and a decrease of sexual activity, which causes personal distress to the woman. Whereas psychological and hormonal hypothesis have been respectively raised to explain sexual disorders, no direct assumptions on their effect on functional brain organization has been made so far. Recent functional neuroimaging studies have however identified clear modifications of cerebral regions during sexual arousal. For instance, activation of the inferior frontal lobe, cingulate gyrus, medial prefrontal, insula gyrus, corpus callosum, thalamus, caudate nucleus, globus pallidus, inferior temporal lobe, as well as the amygdala have been associated with sexual arousal response evoked by visual stimulation in healthy women [Park et al., 2001; Karama et al., 2002; Hamman et al., 2004]. In the same way, some studies in epileptic patients have mainly suggested hyposexuality as being pathogenically related to right hippocampal atrophy [van Elst et al., 2003; Wuerfel et al., 2004]. Our review of functional neuroimaging studies during sexual arousal in women emphasizes the importance to more often take into account the neuro-functional dimension in standard sex therapy. This multidisciplinary approach could indeed provide valuable clinical information pertaining to sexual arousal disorders and other diseases in women. In light of this, we propose a reevaluation of the Twentieth-Century sexual thought on womens' desires.

FNS\#31-65096.01/2.

\section{P I/14 Psychosomatik \\ Schwanger bei unklarer Vaterschaft - Auswege aus dem Dilemma}

Stäubli A.C., Tschudin S., Bitzer J., Holzgreve W.

Universitätsfrauenklinik Basel

Einführung: GynäkologInnen werden in der Beratung häufig mit komplexen Problemen konfrontiert, welche nicht allein mit medizinischem Wissen gelöst werden können, weil sie mit biomedizinisch-ethischen Dilemmata verbunden sind. Anhand eines Fallberichts soll dies dargestellt werden. Fallbericht: Frau B., 22j. türkische GII PI, meldet sich in der $11+2$ Schwangerschaftswoche (SS-W) bei Unklarheit des Kindsvaters. Die Patientin hatte einen einmaligen sexuellen Kontakt mit dem Cousin des Ehemannes um den Zeitpunkt der Konzeption. Die Patientin wünscht eine Vaterschaftsabklärung. Falls der Kindsvater der Cousin des Ehemannes ist, möchte sie die SS abbrechen. Das Ehepaar hat seit zwei Jahren Kinderwunsch. Es besteht zudem häusliche Gewalt. Theoretisch besteht die Möglichkeit, eine Vaterschaftsabklärung bei intakter SS mittels invasiver Diagnostik (IDG) auf Wunsch der Patientin durchzuführen. Bei einer IDG besteht ein Abortrisiko von 1\%. Das Ziel der IDG ist, das Vorliegen von genetischen Erkrankungen nachzuweisen. Der genetische Nachweis des Kindsvaters würde über das Fortbestehen der SS bestimmen. Das Ziel der Beratung ist, die Patientin umfassend zu informieren und im Prozess der Entscheidungsfindung zu unterstützen. Dabei sollen die folgenden Prinzipien der biomedizinischen Ethik eingehalten werden: (1) Die Autonomie der Patientin respektieren; (2) Pflicht der Nonmalefizienz: der Patientin keinen Schaden zuführen; (3) Benefizienpflicht: zum Wohlbefinden der Patientin beisteuern; (4) Gerechtigkeit: gleiche Bedingungen für alle. In zwei ausführlichen Gesprächen wurden zusammen mit der Patientin die möglichen Szenarien - SSAbbruch, Vaterschaftsabklärung postpartum und Aufziehen des Kindes bei unbekanntem Kindsvater - abgewogen und dabei die emotionalen Aspekte ergründet. Unter Berücksichtigung der 4 oben genannten Prinzipien wurde von einer IDG abgeraten. Die Patientin sucht das Gespräch mit dem Cousin des Ehemannes. Dieser bestätigt, die Verhütung durch Coitus interruptus praktiziert zu haben. Daraufhin ist die Patientin sicher, dass der Kindsvater ihr Ehemann ist. Sie möchte die SS austragen. Schlussfolgerung: Die Patientin soll in der Beratung von komplexen Lebenssituationen in ihrer Entscheidungsfindung unterstützt werden, wobei durch die Anwendung biomedizinethischer Prinzipien der Entscheidungsprozess transparent gemacht wird.

\section{P I/15 Psychosomatik \\ Psychosoziale Netzwerke für opioidabhängige Schwangere}

Schossig C., Tschudin S., Bitzer J., Holzgreve W.

Universitätsfrauenklinik Basel

Einführung: Mit zunehmendem Angebot an Substitutionsprogrammen und medizinischer Betreuung bessert sich die gesundheitliche Verfassung opioidabhängiger Frauen im fertilen Alter. So steigt die Zahl der Schwangeren, die aufgrund ihrer Drogenabhängigkeit als Risikoschwangere einzuschätzen sind und in komplexen psychosozialen Belastungssituationen leben. Deren Betreuung und Begleitung stellt eine besondere Herausforderung dar. Ausgehend von einem Fallbeispiel soll das Basler Betreuungsmodell aufgezeigt werden. Fallbericht: Frau A., 32j. I G, stellt sich in der Poliklinik 
in der 10. Schwangerschaftswoche mit der Frage nach Beurteilung ihrer Medikation wegen Depression und Borderlinestörung vor. Die Schwangerschaft ist erwünscht. Die Patientin gibt an, seit 1/2 Jahr nicht mehr opioidabhängig zu sein. In einem ersten Schritt bietet sich die betreuende Ärztin aus der Abteilung für gynäkologische Psychosomatik und Sozialmedizin als Bezugsperson und Koordinatorin verschiedener Spezialdienste an. Bei der Ankündigung, ein Routine-Urinscreening auf Opiate durchzuführen, berichtet Frau A. aktuellen Konsum diverser Opioide. Diesen hatte sie aus Angst, dass ihr das Kind weggenommen werden könnte, zu verheimlichen versucht. Unter Einbeziehung unseres Sozialdienstes und Kontaktaufnahme mit der psychiatrischen Poliklinik gelingt es, die Patientin sofort in ein Methadonprogramm aufzunehmen. Die Patientin wird zur Absprache der postpartalen Betreuung den Neonatologen vorgestellt. Unter Einbeziehung der verschiedenen Spezialdienste gelingt es, die Schwangerschaft ohne geburtshilfliche Komplikationen bis zum errechneten Termin zu bringen. Bei Aufnahme zur primären Wunschsectio kommt Frau A. mit vollständig eröffnetem Muttermund und gebiert spontan ein reifes, normgewichtiges Mädchen, das primär gut adaptiert und zur Überwachung auf die Neonatologie aufgenommen wird. Ab dem vierten Lebenstag zeigt das Neugeborene Entzugssymptome, die erfolgreich behandelt werden. Nach Absprache mit den Eltern wird dem Kind über die Abteilung für Kinder- und Jugendschutz ein Betreuer zur Seite gestellt. Schlussfolgerung: Gerade die Betreuung opioidabhängiger Schwangerer ist personell sehr aufwendig und erfordert viel interdisziplinäre Koordination und Kommunikation. Damit ist es möglich, zu einem befriedigenden Outcome für Mutter und Kind zu kommen.

\section{P I/16 Psychosomatik \\ Interruption de grossesse chez les migrantes 'sans papiers': Destin ou conséquence de la détresse socio-économique?}

Bloch A., Hohlfeld P., Renteria S.-C.

Département de Gynécologie et d'Obstétrique, Centre

Hospitalier Universitaire Vaudois, Lausanne

Introduction: Le taux d'IVG est plus élevé chez les migrantes $(16,9 \%)$ que chez les Suissesses $(5,7 \% 0)$ selon les statistiques du canton de Vaud (2002). Des programmes de prévention y relatifs sont en voie d'élaboration. Les statistiques officielles ne détiennent pas d'informations concernant la situation des migrantes sans permis d'établissement, les «sans papiers» («SP»), non recensées par l'administration. Le but est d'analyser leur problématique et de détecter des possibles liens de causalité ainsi que des pistes pour améliorer la situation des femmes concernées. Matériel et méthodes: (1) Calcul du taux d'IVG chez les «SP» (statistique DGO et étude de la Municipalité de Lausanne, 2001). (2) Analyse rétrospective des IVG au DGO (relais pour une majeure partie des «SP») entre 2003 et 2004 chez les «SP» et les migrantes avec permis d'établissement. Resultats: Taux d'IVG «SP»: 2003: 20-50\%0; 2004: 16,6-41,5\% . IVG au DGO: «SP» $(\mathrm{n}=183)$ versus autres migrantes $(\mathrm{n}=641)$. Origine: Amérique du sud $(60,4$ vs. $7,2 \%)$, Afrique $(15,9$ vs. $31,7 \%)$. Activité lucrative régulière (4,9 vs. $23,7 \%)$. Contraception au moment de la conception: aucune (33,3 vs. $23,4 \%)$, orale ( 8,7 vs. $16,7 \%)$, préservatif (14,8 vs. $16,8 \%)$. Age gestationnel $>12$ sem $(9,3$ vs. $6,1 \%)$. Motif pour l'IVG: économique: $76 \%$ vs. $72,1 \%$; lié au partenaire 22,4 vs. $19,7 \%$. Assurance maladie 5,5 vs. $100 \%$ (couverture partielle des frais par une fondation privée dans $16,8 \%$, par patiente/partenaire dans $77,7 \%$ des cas). Conclusions:
Le taux d'IVG est plus élevé chez les «SP» que chez l'ensemble des migrantes. Les raisons d'une conception non désirée et d'une interruption de grossesse parlent en faveur d'un lien direct avec la détresse sociale, l'accès insuffisant au système de santé et par conséquent à la contraception. En raison de l'absence d'assurance, l'IVG a pour ces migrantes des conséquences psychologiques mais surtout financières désastreuses. Des stratégies de prévention pourraient englober la création de consultations bas seuil et la mise à disposition de méthodes contraceptives bon marché, comme c'est déjà établi pour les requérantes d'asile du canton de Vaud. La réduction des frais hospitaliers non couverts pourrait compenser une partie de ces dépenses.

\section{P I/17 Psychosomatik \\ Violence sexuelle à I'adolescence: L'agresseur n'est pas toujours un inconnu}

Renteria S.-C., Savary M.-C., De Grandi P.

Département de Gynécologie et d'Obstétrique,

Centre Hospitalier Universitaire Vaudois, Lausanne

Introduction: La prise en charge adéquate d'adolescentes victimes de violences sexuelles doit se baser sur la connaissance des caractéristiques de ces situations. Matériel et méthode: Analyse retrospective quantitative et qualitative basée sur l'anamnèse et le constat chez les victimes d'agression sexuelle, âgées de 14 à 20 ans, consultant entre janvier 2001 et 2005. Résultats: Les données de 78 adolescentes, âge moyen 16,6 ans, consultant en vue d'un constat d'agression sexuelle (39 après pénétration pénienne) ont fait l'objet d'un relevé informatique durant cette période. 17 adolescentes avaient déjà eu des rapports sexuels antérieurs avec consentement. L'examen gynécologique a permis de documenter des modifications morphologiques chez 39\% des filles sans activité sexuelle antérieure (modif. spécifiques 17; non spécif. 7). Trois présentaient une infection sexuellement transmissible. 51,3\% des filles connaissaient leur agresseur (bien que non issu du cadre familial). Elles avaient rendez-vous («date rape») ou avaient fait connaissance lors d'un sortie entre amis. Les récits des adolescentes font état d'un décalage flagrant entre les attentes de la victime (discussion, échange de tendresse, parfois même activité sexuelle) et les interprétations et actes des agresseurs. L'agression sexuelle est souvent précédée d'échanges sociaux prolongés malgré l'insécurité des lieux et des circonstances (lieux isolés, appartement de l'agressseur, consommation d'alcool, heure tardive). Ce vécu provoque à tord un sentiment de culpabilité chez les jeunes filles, souvent associé à la peur d'une réaction parentale négative, ce qui leur rend difficile la déposition d'une plainte. Conclusions: Les adolescentes victimes de violence sexuelle ont souvent établies des liens sociaux, parfois même de confiance, avec l'agresseur (amis communs, sentiment amoureux) dans les jours ou mois qui précèdent l'agression. Il leur est dès lors d'autant plus difficile de décéler le danger et d'interpréter les signes annonciateurs de violence. La prise en charge des victimes et l'accompagnement auprès des instances judiciaires doivent tenir compte de ces circonstance particulières ainsi que de leur fréquence. Une sensibilisation préventive auprès des jeunes et des parents paraît également indispensable.
Abstracts, gynécologie suisse, Lugano, 2005 


\section{P II/20 Onkologie \\ Métastase cervical d'un cancer du sein}

Locca Y. ${ }^{\mathrm{a}}$, Pelte M.-F. ${ }^{\mathrm{b}}$, Vlastos G. ${ }^{\mathrm{a}}$, Dubuisson J.B. ${ }^{\mathrm{a}}$,

Vlastos A.-T.

aDDépartement de Gynécologie et d'Obstétrique,

bépartement de Pathologie, Hôpitaux Universitaires de Genève

Introduction: La métastase cervicale d'un cancer du sein est un événement extrêmement rare. La littérature décrit 3 cas de métastase cervicale isolée, et 24 cas entrant dans le cadre de tumeurs multimétastastiques. Nous reportons ici un cas associé à des métastases osseuses. Case Report: Il s'agit d'une patiente de 67 ans, ménopausée depuis 17 ans, sans substitution hormonale, connue depuis 2000 pour un carcinome canalaire invasif du sein droit, G1, pT1c pN1 biii M0, recepteurs positifs (oestrogènes $>95 \%$, progestérone $>95 \%$, MiB $110 \%$ des cellules tumorales, HER 2 positif) traitée par tumorectomie et curage axillaire droit suivie d'une radiothérapie et d'une hormonothérapie (Tamoxifène). La patiente n'a pas bénéficiée d'une chimiothérapie en raison de trouble psychiatrique (schizophrénie paranoïde). 4 ans plus tard de multiples métastases osseuses sont objectivées par scintigraphie, raison pour laquelle le Tamoxifène est remplacé par un traitement d'anti-aromatase (Femara). Sur le plan cervical, en novembre 2001, le frottis de dépistage met en évidence une lésion de bas grade (LSIL), avec présence d'un Human Papilloma Virus oncogénique, confirmée histologiquement. La lésion reste stable mais en Octobre 2003, le PAP test montre des cellules squameuses atypiques avec une lésion de haut grade à exclure (ASC-H) et des cellules glandulaires atypiques (AGUS) dont les bilans (curetage de l'endocol, hystéroscopie et pipelle de Cornier) s'avèrent non concluants. En raison de la persistance d'un ASC-H, une conisation est effectuée en Janvier 2005. L'analyse histologique montre une lésion de bas grade (CIN 1) ainsi qu'une lésion de haut grade (CIN 2) enlevées in toto. On note en outre une infiltartion carcinomateuse à cellules isolées, PAS et bleu Alcian positives, cytokératines totales et CK7 positives, recepteurs d'oestrogènes et de progestérone positives, compatible avec une origine mammaire. Conclusion: La métastatisation cervicale d'un cancer du sein étant très rare, l'attitude thérapeutique est difficile. Dans la littérature cependant, un traitement agressif sous forme d'hystérectomie avec annexectomie est indiquée en cas de bilan d'extension négatif.

\section{P II/21 Onkologie \\ Verminderung des zytotoxischen Effektes von Cisplatin und Lipoplatin durch Protein Kinase B (AKT)-Hemmer in Abhängigkeit von DNA Mismatch Repair}

Fedier A., Schuchter U., Fink D.

Klinik für Gynäkologie, Departement Frauenheilkunde, Universitätsspital Zürich

Einführung: Eine wichtige Funktion der Akt/PKB (Protein Kinase B)-vermittelten Signaltransduktion ist die Kontrolle der Zellproliferation und der Apoptose. Diese Signalkaskade ist in Tumorzellen oftmals (über-)aktiviert, was zur verstärkten Proliferation und Hemmung der Apoptose führen kann. Die Blockierung von Akt/PKB durch spezifische Inhibitoren führen in Tumorzellen zu einer Erhöhen der Chemo- und Radiosensitivität. Ziel dieser Arbeit war es zu untersuchen, ob die für DNA Mismatch Repair (MMR)defiziente Tumorzellen charakteristische Chemoresistenz (z.B.
Cisplatin) durch spezifische Akt/PKB-Hemmer aufgehoben werden kann. Material und Methode: MMR-defiziente (HCT116+ch2) und MMR-profiziente (HCT116+ch3) Kolontumorzellen wurden verwendet. Mittels des ‘Clonogenic Assay' wurde die Sensitivität von Tumorzelllinien gegenüber diversen Chemotherapeutika alleine mit derjenigen gegenüber einer Kombination dieser Chemotherapeutika mit dem Akt/PKB-spezifischen Hemmer LY294005 verglichen. Ergebnisse: Die Ergebnisse zeigten, dass LY294005 die Sensitivität der beiden Zelllinien gegenüber Cisplatin und Lipoplatin nicht erhöht, sondern vielmehr vermindert. Interessanterweise war diese Reduktion in MMR-profizienten Zellen klar ausgeprägter (bis zu 2-fach) als in MMR-defizienten Zellen. Da Cisplatin und Lipoplatin eine MMR-abhängige Wirkungsweise haben, liess dieses Resultat einen Zusammenhang zwischen MMR und Akt/PKB vermuten. Die Ergebnisse mit MMR-unabhängigen Chemotherapeutika (Oxaliplatin, Docetaxel) zeigten, dass LY294005 die Sensitivität von Tumorzellen gegenüber diesen zwar ebenfalls verminderte, aber gleichermassen in MMR-profizienten und MMR-defizienten Zellen. Dies bestätigt die oben geäusserte Vermutung nach einer möglichen Verbindung zwischen MMR und Akt/PKB. Schlussfolgerung: Anders als erwartet verminderte der spezifische Akt/PKB-Hemmer LY294005 den zytotoxischen Effekt der getesteten Chemotherapeutika, anstatt ihn zu erhöhen. Interessant ist, dass dieser Effekt abhängig ist vom MMR-Status der Zellen. Dies lässt einen funktionellen Zusammenhang zwischen der Akt/ PKB-Signalkaskade und der MMR-vermittelten Erkennung von DNA-Schädigungen vermuten.

\section{P II/22 Onkologie \\ Discriptive Epidemiology of Vaginal Cancers in Geneva, Switzerland, 1970-2002: Preliminary Results}

Botta U. ${ }^{\mathrm{a}}$, Usel M. ${ }^{\mathrm{b}}$, Neyrond I. ${ }^{\mathrm{b}}$, Bouchardy $\mathrm{C}^{\mathrm{b}}$, Dubuisson J.B. ${ }^{\mathrm{a}}$, Vlastos A.T. ${ }^{\mathrm{a}}$

aDepartment of Gynecology and Obstetrics, Geneva University Hospital, ${ }^{\mathrm{b}}$ Geneva Tumor Register

Introduction: Vaginal cancers (VC) are rare accounting for $1 \%$ of women cancers and $9 \%$ of gynaecological cancer. The disease typically, in situ VC develops before the 7th decade and invasive cancer after. Material and Methods: To analyse trends in incidence, for the in situ and invasive VC using data from the Geneva Cancer Registry prospectively collected over the past 32 years (1970-2002). Results: 81 VC were reviewed, $36(44,4 \%)$ in situ and $45(55,6 \%)$ invasive. Median age was 52,9 years for the in situ cancer and 65,2 years for the invasive cancer; difference was 12,3 years. 15 patients less than 50 were diagnosed with in situ VC and 7 patients over 70 were diagnosed with in situ VC. Same way, 22 patients over 70 years were diagnosed with invasive VC, whereas 12 patients under 50 were diagnosed with invasive VC. Trend in incidence showed a decreased in invasive VC incidence (19 in the period 1978-1985 to 7 in the period 1995-2002). The incidence of in situ VC increased from 4 in the period 1978-1985 to 27 in the period 1995-2002. These population-based results showed that incidence of invasive $\mathrm{VC}$ seems to be decreasing over the past 32 years $(p=0,67)$, and in situ VC increasing $(p=0,051)$. Conclusion: $\mathrm{VC}$ are rare tumours involving mostly patients over 50 years old for the in situ VC and after 70 years old with invasive VC. With aging population invasive $\mathrm{VC}$ will become probably more frequent; however, to reach statistical significance we need a bigger cohort to confirm this trend. 


\section{P II/23 Onkologie \\ Laparoskopische Hysterektomie - Ist die Einführung einer neuen Methode im Nicht-Zentrumsspital zulässig?}

Rautenberg W., Christoffel L., Honegger Ch.

Frauenklinik, Spital Uster

Einführung: Mit der laparoskopischen Hysterektomie wurde eine Operationsmethode gefunden, deren Morbidität und Mortalität das Niveau der vaginalen Hysterektomie erreicht und Indikationen der abdominellen Hysterektomie abdeckt. Mit den vorliegenden Daten soll gezeigt werden, ob die Einführung dieser neuen operativen Methode an einem Nicht-Zentrumsspital mit einer vertretbaren Morbidität einhergeht. Material und Methoden: Wir berichten von 28 laparoskopischen Hysterektomien, die zwischen 08/2003 und 01/2005 in der Frauenklinik des Spitals Uster, einem Haus der erweiterten Grundversorgung, durchgeführt wurden. Bei den 30-57 Jahre alten Patientinnen bestanden Indikationen, die eine deutlich erschwerte oder keine vaginale Hysterektomie ermöglicht hätten. Alle Operationen wurden vom gleichen Operateur durchgeführt; es wurde ein Uterusmanipulator Modell Clérmont Ferrand und das bipolare Koagulationssystem von ERBE verwendet. Ergebnisse: Die Indikationen waren in 17 Fällen ein symptomatischer Uterus myomatosus, in 5 Fällen invalidisierende Dysmenorrhoe bei Endometriose und Nulligravidität, 3mal Blutungsstörungen bei komplexer Endometriumshyperplasie, in 2 Fällen einfache Blutungsstörungen und $1 \mathrm{mal}$ bekannte Adhäsionen. Die durchschnittliche Operationszeit lag bei $142 \mathrm{~min}$ (90-240 min), der Spitalaufenthalt bei 5,9 Tagen (3-9 Tage) und das Uterusgewicht

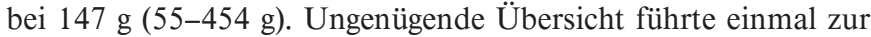
Konversion auf eine abdominelle Hysterektomie. Schwere Komplikationen traten in 4 Fällen auf: eine eingeblutete Ovarialzyste mit Begleitperitonitis, eine Narbenhernie und eine dehiszente Kolpotomie. Eine Blasenläsion konnte intraoperativ laparoskopisch versorgt werden. Leichte Komplikationen: 2 Fälle von nicht transfusionsbedürftiger Anämie, 2 Fälle mit Harnwegsinfekt. Schlussfolgerung: Im Vergleich zur Literatur war die operative Morbidität in unserer Serie von 28 Fällen nicht erhöht. Entsprechend erscheint die Einführung einer neuen Operationsmethode bei adäquater Infrastruktur und allgemein operativer Erfahrung gerechtfertigt.

\section{P II/24 Onkologie \\ Effekt einer mehrmaligen Behandlung von Tumorzellen mit den Histon- Deazetylasen-Hemmern SAHA und TSA auf die Chemosensitivität}

Dedes K.J., Imesch P., Fink D., Fedier A.

Klinik für Gynäkologie, Departement Frauenheilkunde, Universitätsspital Zürich

Einführung: Histon-Deazetylasen (HDAC)-Hemmer beeinflussen die Expression u.a. von Tumor-assoziierten Genen, induzieren Apoptose in Tumorzellen und gelten somit als potentielle Chemotherapeutika. Ziel der Studie war es zu untersuchen, ob die mehrmalige Behandlung von Tumorzellen mit den HDAC-Hemmern SAHA und Trichostatin A (TSA) die Sensitivität dieser Tumorzellen einerseits gegenüber HDAC-Hemmern selber und andererseits gegenüber anderen Chemotherapeutika beeinflusst. Material und Methode: Verwendet wurden menschliche Kolontumorzellen entweder profizient $(\mathrm{HCT} 116+\mathrm{ch} 3)$ oder defizient
(HCT116+ch2) in der DNA Mismatch Repair (MMR)-Funktion, sowie die entsprechenden Sublinien, «selektioniert» durch 10-maliges Behandeln der (primären) Zelllinien mit ansteigenden Dosen von SAHA. Die Sensitivität gegenüber SAHA, TSA und anderen Chemotherapeutika wurde durch den «Clonogenic Assay» bestimmt. Ergebnisse: Die primären MMR-profizienten und die MMR-defizienten Zellen wiesen eine vergleichbare Sensitivität gegenüber SAHA und TSA auf. Die «Selektion» mit SAHA führte zu einer Reduktion der Sensitivität gegenüber sowohl SAHA als auch TSA. Diese Reduktion war in MMR-profizienten und MMR-defizienten Zellen von vergleichbarem Ausmass (2-fach) und damit unabhängig vom MMR-Status. Hingegen bewirkte die «Selektion» eine 2-fache Erhöhung der Sensitivität gegenüber Oxaliplatin in MMR-profizienten Zellen, aber nicht in MMR-defizienten Zellen. Zudem bewirkte sie eine 2-fache Reduktion der Sensitivität gegenüber Lipoplatin in MMR-profizienten Zellen, dagegen aber eine 2-fache Erhöhung der Sensitivität in MMR-defizienten Zellen. Schlussfolgerung: Die Studie zeigt, dass die zytotoxische Wirkung der HDAC-Hemmer SAHA und TSA auf eine Weise erfolgt, die unabhängig vom MMR-Status der Zellen ist. Beide könnten damit für die Behandlung von Tumoren mit MMR-Genmutationen in Betracht gezogen werden. Die Studie zeigt aber auch die Möglichkeit auf, dass die repetitive Behandlung von Tumorzellen mit hohen Dosen von HDAC-Hemmern die Chemosensitivität beeinflussen kann. Grund dafür könnte eine durch die Behandlungen bedingte nachhaltige Veränderung von Expressionsmustern von u.a. Tumor-assoziierten Genen sein. Dies könnte von klinischer Bedeutung sein.

\section{P II/25 Onkologie \\ Tumeur du sac vitellin de l'ovaire: Un défi diagnostic pour une tumeur rare - A propos d'un cas et prise en charge actuelle Dällenbach $P^{\mathrm{a}}$, Pelte M..$^{\mathrm{b}}$, Vlastos $G .^{\mathrm{a}}$ \\ aService de Gynécologie, 'b Service de Pathologie Clinique, Hôpitaux Universitaires de Genève}

Introduction: Les tumeurs du sac vitellin de l'ovaire sont des néoplasies malignes rares de la femme jeune de diagnostic difficile et déterminant. Description d'un cas: Une femme âgée de 22 ans se présenta avec des douleurs pelviennes intermittentes, accompagnées de vomissements et de fièvre $\left(38,9^{\circ} \mathrm{C}\right)$. La palpation révéla une masse pelvienne de $12 \mathrm{~cm}$ de diamètre, et l'imagerie une masse annexielle hétérogène de $10 \times 8 \mathrm{~cm}$ compatible pour le radiologue avec une nécrobiose d'un fibrome ou un abcès tubo-ovarien. A part une CRP élevée, les tests sanguins étaient normaux. En l'absence d'amélioration clinique malgré une antibiothérapie bien conduite, une laparoscopie fut décidée avec excision d'une tumeur solide d'aspect bénin terminée par laparotomie en raison d'un saignement. L'histologie révéla une tumeur maligne de l'ovaire dont certains aspects évoquaient une tumeur à cellules de la granulosa, et d'autres une tumeur du sac vitellin. Les coupes furent revues dans une autre institution et le diagnostic de tumeur du sac vitellin fut confirmé. L'AFP et le CA 125 complémentaires étaient très élevés. Le bilan d'extension ne montra pas de métastases. La patiente bénéficia de 3 cycles de chimiothérapie type BEP (Bléomycine, Etoposide, Cisplatine). A 6 mois de suivi, elle était en rémission avec normalisation des marqueurs sériques. Discussion: Les tumeurs du sac vitellin appartiennent au groupe des tumeurs des cellules germinales qui représentent 3 à $5 \%$ de tous les cancers ovariens, mais constituent la majorité des cancers ovariens de l'enfant et de la femme jeune (60\%). Elles produisent l'AFP, dont le dosage séri-
182

Gynäkol Geburtshilfliche Rundsch 2005;45:165-221
Abstracts, gynécologie suisse, Lugano, 2005 
que prédit la persistance ou la récidive de la maladie. Comme illustré par notre cas, le diagnostic peut être difficile à la fois pour le clinicien, le radiologue et le pathologue. Avant l'utilisation de la chimiothérapie adjuvante, l'issue était presque toujours fatale (survie à 5 ans entre 5 et $20 \%$ pour les stades I) malgré une chirurgie oncologique complète. Avec le régime BEP recommandé actuellement, la survie atteint $95 \%$ et la plupart des femmes peuvent bénéficier d'une chirurgie préservant la fertilité. Conclusion: Ce cas souligne l'importance de connaître et d'identifier cette tumeur rare quand le clinicien est confronté à une masse pelvienne de croissance rapide chez une femme jeune.

\section{P II/26 Onkologie \\ Laparoskopische Therapie bei Borderline- Tumoren des Ovars}

Matthey M.-L. ${ }^{a}$, Sonnenschein M. ${ }^{a}$, Dreher E. ${ }^{a}$,

Mueller M.D. ${ }^{\mathrm{a}, \mathrm{b}}$, Bratschi H.U. ${ }^{\mathrm{b}}$, Eggimann T. ${ }^{\mathrm{b}}$, GyrTh. $^{\mathrm{b}}$,

Lüscher K.P. ${ }^{\mathrm{b}}$, Steiner R. ${ }^{\mathrm{b}}$, Stucki $D .^{\mathrm{b}}$

aUniversitäts-Frauenklinik, Inselspital Bern,

${ }^{\mathrm{b}}$ Arbeitsgemeinschaft für Endoskopische Gynäkologie

Einführung: Ziel der Arbeit war, das unmittelbare Outcome nach laparoskopisch operierten Borderline-Tumoren des Ovars, im Vergleich zur Laparotomie, zu analysieren. Material und Methode: Retrospektive Analyse von 66 Fällen von Borderline-Tumoren des Ovars, welche zwischen 1990 und 2005 in 6 verschiedenen schweizerischen Frauenkliniken operiert wurden. Entsprechend dem Operationsverfahren wurden zwei Gruppen gebildet: Laparoskopie(LSC) resp. Laparotomie (LT). Ergebnisse: Das präoperativ abgenommene CA-125 war bei 31 (47\%) Patientinnen im Normbereich. $20(30,3 \%)$ Patientinnen wurden initial per LSC, 46 $(69,7 \%)$ per LT operiert. $23(34,8 \%)$ der Patientinnen hatten Kinderwunsch (KW), davon 10 (50\%) der LSC und 13 (28\%) der LTGruppe. Das durchschnittliche Alter der Patientinnen mit LSC lag bei 35,6 \pm 9,9 J., mit LT bei 48,3 \pm 17,8 Jh. Alle Patientinnen über $60 \mathrm{~J}$. wurden primär laparotomiert $(\mathrm{n}=12)$. Die laparoskopischen Eingriffe umfassten $10(50 \%)$ ein- oder beidseitige Adnexektomien und $10(50 \%)$ Zystektomien. In $3(30 \%)$ dieser Fälle kam es intraoperativ zur Zystenruptur. Bezüglich Histologie (51\% serös, 46\% muzinös, $2 \%$ klarzellig, $2 \%$ endometrioid) und Tumorstadium (FIGO Ia $62,1 \%$, Ib $9,1 \%$, Ic $15,2 \%$, II $6,1 \%$, III 7,5\%) bestand zwischen beiden Gruppen kein Unterschied. Nach LSC musste in 4 (20\%) Fällen eine Zweitlaparoskopie und in 12 (60\%) Fällen eine Laparotomie durchgeführt werden. Eine Patientin $(2,2 \%)$ mit LT wurde relaparotomiert und $4(8,7 \%)$ wurden, zur Vervollständigung des Stagings, nach einem Intervall von $>6$ Wochen, laparoskopiert. Die Hospitalisationsdauer bei LSC betrug im Mittel 7,4 $\pm 6,3$, bei LT $12,1 \pm 4,8$ Tage. In der LSC-Gruppe wurde keine Komplikation festgestellt, in 6 (13\%) Fällen der LT-Gruppe sind Komplikationen aufgetreten. Schlussfolgerung: Bei geringerer Komplikationsrate und kürzerer Hospitalisationszeit ist die LSC bei Patientinnen mit KW, wo ein konservatives Vorgehen geplant ist, indiziert. Präoperativ müssen die Patientinnen über den hohen Anteil an Zweiteingriffen informiert werden. Vor einer definitiven Aussage muss das Langzeit-Follow-up abgewartet werden, denn nur dieses wird zeigen, ob die Prognose durch die LSC nicht verschlechtert wird.

\section{P II/27 Onkologie \\ Galectin 4 und Meprin A als neue \\ Tumormarker für das muzinöse \\ Ovarialkarzinom}

\begin{abstract}
Heinzelmann-Schwarz V.A. ${ }^{\text {a, }}$, Gardiner-Garden M. ${ }^{a}$ Henshall S.M. ${ }^{\text {a }}$, Scurry J. ${ }^{\text {, }}$ Scolyer R.A. ${ }^{\mathrm{c}}$, Fink D. ${ }^{\mathrm{d}}$, Sutherland R.L. ${ }^{\mathrm{a}}$, Hacker N.F. ${ }^{\mathrm{e}} \mathrm{O}^{\prime}$ Brien P.M. ${ }^{\mathrm{a}}$

${ }^{a}$ Cancer Research Program, Garvan Institute of Medical Research, Darlinghurst, NSW, 'bouth Eastern Area Laboratory Service, Prince of Wales Hospital, Randwick, NSW, 'Department of Anatomical Pathology, Royal Prince Alfred Hospital, Camperdown, NSW, Australia, ${ }^{d}$ Klinik für Gynäkologie, Departement Frauenheilkunde, Universitätsspital Zürich, ${ }^{e}$ Gynaecological Cancer Centre, Royal Hospital for Women, Randwick, NSW, Australia
\end{abstract}

Einführung: Etwa 14\% der Ovarialkarzinome sind vom muzinösen Subtyp (MOC). Klinisch, morphologisch und epidemiologisch stellt das MOC eine Entität dar, welches durch den aktuellen Tumormarker CA125 nicht entdeckt werden kann. Differentialdiagnose zum muzinösen Borderline-Tumor sowie zu intestinalen muzinösen Metastasen im Ovar ist schwierig, aber von grosser Bedeutung. Material und Methoden: Mittels eines $>59$ 000-Oligonukleotid-Microarrays, der $>90 \%$ des menschlichen Genoms enthält, untersuchten wir das genetische Profil des MOC an 51 unterschiedlichen Ovarialkarzinomen und 4 normalen Ovarien. Unser Ziel war die Darstellung der genetischen Basis des MOC sowie die Identifizierung potentieller Tumormarker für Früh- und Differentialdiagnose. Ergebnisse: Wir konnten ein spezifisches genetisches Profil für das MOC identifizieren, welches zu den anderen Subtypen sowohl Überlappungen als auch Gegensätze zeigt. Die Expression zweier der 167 identifizierten Gene wurde mittels Immunhistochemie an "Tissue Microarrays», welche aus 156 Tumoren bestehen, weiter untersucht. Die beiden Kandidaten Galectin 4 (LGALS4) und Meprin A (MEP1A) zeigten eine hoch signifikante MOC-spezifische Expression. LGALS4 konnte zudem auch in benignen Ovarien sowie muzinösen Borderline-Tumoren festgestellt werden, was die Hypothese eines Progressionsmodels für das MOC stützt. MEP1A hingegen wird stärker in muzinösen intestinalen Karzinomen exprimiert, was von grosser differentialdiagnostischer Bedeutung ist. Schlussfolgerung: LGALS4 und MEP1A zeigen eine stärkere Differenzierung zwischen MOC und muzinösen intestinalen Karzinomen als die aktuell in der Pathologie benützten Marker CK7 und CK20. Zusätzlich hat das sezernierte LGALS4 grosses Potential als Serummarker zur Frühentdeckung und Differentialdiagnose des MOC.

\section{P II/28 Onkologie \\ Laparoscopic Surgery of Cervical Carcinoma}

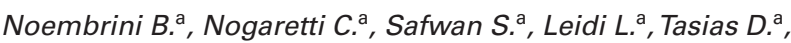
Gianoni A. ${ }^{\mathrm{a}}$, Richetti A. ${ }^{\mathrm{b}}$, Sessa Ch. ${ }^{\mathrm{b}},{ }$ GyrTh. ${ }^{\mathrm{a}}$

${ }^{a}$ Reparto di Ginecologia Ostetricia, Ospedale Civico, Lugano and ${ }^{\mathrm{b}}$ Istituto di Oncologia della Svizzera Italiana, Bellinzona

Introduction: Laparoscopy has been shown to be advantageous for patients with endometrial carcinoma. Laparoscopy has also been suggested for the staging and treatment of patients with cervical cancer. We report 11 patients treated in laparoscopy at our institution between November 02 and January 05. Materials and Methods: Patients were submitted to laparoscopical staging after clinical workup including cysto- and rectoscopy. Staging included 
pelvic lymphadenectomy and biopsy of the cardinal and sacrouterine ligaments if parametrial involvement was suspected. If frozen section was negative, total laparoscopic radical hysterectomy (Piver II/III) was performed as described for laparotomy with the exception that ultracision dissection was used. If frozen section was positive, patients were referred to combined radio- and chemotherapy. Results: The mean age was 52.3 years (30-86). In 5 cases, staging revealed positive lymph nodes or parametrial involvement. One of these patients showed stage FIGO Ib1 and 4 patients $>\mathrm{Ib}$ disease. In 6 cases with FIGO stage Ia2 (2), Ib1 (3) and IIb (1) radical hysterectomy (Wertheim) was performed in laparoscopy. In this group, operating time was $250 \mathrm{~min}$ (210-600), blood loss was $400 \mathrm{ml}$ (200-800), 19.2 (13-29) lymph nodes were examined. Beginning parametrial involvement was found in 1 case with $2 / 21$ positive lymph nodes. All patients were fully mobile and ate on the first postoperative day. Complications were 2 urinary infections, no febrile morbidity was observed. One patient of the staging group was reoperated after 6 days for painful lymphocele. We did not have any conversion to laparotomy. Postoperative hospital stay was 8 days (4-15), due to bladder draining. Conclusion: Laparoscopy is an excellent tool for accurate staging and radical operation of patients with cervical cancer. Parametrial cuff, number of lymph nodes examined and operating time are similar to laparotomy. With increasing experience of the operating team, laparoscopy will probably replace laparotomy in the treatment of this disease.

\section{P III/30 Urogynäkologie \\ Der Kontinenzmechanismus des TOT: Eine Perinealsonographische Studie}

Troeger C., Humburg J., Holzgreve W., Wight E.

Universitäts-Frauenklinik Basel

Einführung: Von Studien nach TVT-Einlage ist bekannt, dass die Position und Mobilität des Bandes wichtig für die postoperative Kontinenz sind. Das TOT hat ähnlich gute Erfolgsraten, aber über den Kontinenzmechanismus gibt es bislang keine Daten. Material und Methode: 15 Frauen (56 Jahre, 39-86) mit Belastungsinkontinenz ohne signifikanten Deszensus wurden präoperativ mittels Urodynamik und Perinealsonographie(US) evaluiert. Beim US wurden Dx und Dy gemessen und daraus der Bewegungsvektor berechnet: $\mathrm{v}=\mathrm{SQR}(\Delta \mathrm{Dx} 2+\Delta \mathrm{Dy} 2)$. Das TOT erfolgte als singulärer Eingriff. 8 Monate postoperativ (3-16 Monate) erfolgte eine klinische Nachuntersuchung inklusive US. Ergebnisse: 13 von 15 Frauen waren postoperativ kontinent. Bei diesen war die Vektormobilität des Blasenhalses unverändert (10,5 vs. 7,9 mm). Das TOT lag über der zentralen Symphysenlinie sowohl in Ruhe (Dy = $3,1 \mathrm{~mm}$ ), als auch beim Pressen (Dy $=0,9 \mathrm{~mm}$ ). Bei den Frauen mit postoperativ persistierender Inkontinenz dagegen nahm die Mobilität des Blasenhalses signifikant zu (5,2 vs. 15,9 mm). Auch das TOT zeigte eine Hypermobilität in der vertikalen Achse (Dy in Ruhe $=7,8 \mathrm{~mm}$; Dy beim Pressen $=-2,2 \mathrm{~mm}$ ). Hingegen blieb bei allen Patientinnen die Position des Bandes in der x-Achse unverändert (Dx in Ruhe $=23,3 \mathrm{~mm}$; Dx beim Pressen $=23,2 \mathrm{~mm}$ ). $\mathrm{Im}$ gesamten Patientenkollektiv traten keine Komplikationen auf. Schlussfolgerungen: Das TOT ist ebenso effektiv zur Behandlung der Belastungsinkontinenz wie das TVT mit weniger Komplikationsrisiko. Der Erhalt der Mobilität des Blasenhalses und die Immobilität des Bandes vor allem in der y-Achse sind wichtig für die postoperative Kontinenz. Jede Hypermobilität des Blasenhalses und des Bandes hervorgerufen durch einen Deszensus und ein zu locker eingelegtes TOT verschlechtert das Ergebnis.

\section{P III/31 Urogynäkologie Erfahrungen aus Schaffhausen mit dem transobturatorischen Safyre ${ }^{\mathrm{TM}}$-Band}

Tresselt C., Eberhard M., Litschgi $M$.

Klinik für Gynäkologie und Geburtshilfe,

Kantonsspital Schaffhausen

Einleitung: Safyre ${ }^{\mathrm{TM}}$ ist ein neues nachjustierbares, minimal invasives Band für die Therapie der Stressinkontinenz (SUI). Bei der Einlage des transobturatiorschen Bandes (TOT) besteht ein geringes Verletzungsrisiko für Urethra, Blase, gastrointestinale Strukturen und Gefässe. Es stehen bereits verschiedene Methoden zur Wiederherstellung des suburethralen Halteapparates unter Imitierung der anatomischen Strukturen zur Verfügung. Die Autoren berichten über die Erfahrungen mit dieser neuen Methode, welche die Wirksamkeit eines Polypropylenbandes mit der zusätzlichen Möglichkeit zur erneuten Adjustierung vereinigt. Material und Methoden: Insgesamt wurde bei 60 konsekutiven Patientinnen mit der klinischen und urodynamischen Diagnose einer SUI eine TOT-Operation im Zeitraum von März 2003 bis November 2004 durchgeführt. Bei $85 \%$ erfolgte eine isolierte Inkontinenzoperation, bei $15 \%$ wurden zusätzliche gynäkologische Operationen durchgeführt. Das Durchschnittsalter betrug 63 Jahre (36-83 Jahre). Vor der Operation erfolgte eine klinische Untersuchung, ein Stress-Test sowie eine urodynamische Abklärung. Alle Patientinnen zeigten Symptome einer SUI, bei $45 \%$ bestand eine gemischte Inkontinenz. Ergebnisse: Das durchschnittliche Follow-up betrug 8 Monate (221 Monate). Die postoperative Beurteilung der Patientinnen erfolgte anhand der Anamnese und einer klinischer Untersuchung. Die durchschnittliche Hospitalisationsdauer betrug 2,8 Tage. Bei 3\% $(2 / 60)$ kam es intraoperativ zu einer Blasenperforation, eine Patientin entwickelte postoperativ einen perinealen Abszess. Während der Nachbeobachtungszeit waren 82\% (49/60) kontinent, $16 \%$ $(10 / 60)$ berichteten über eine Verbesserung und $2 \%(1 / 60)$ über unveränderte Symptome. Bei 2 Patientinnen erfolgte eine erneute Justierung des Bandes, anschliessend zeigte sich eine vollständige Kontinenz. Insgesamt kam zu einer hochsignifikanten Verbesserung $(p<0,0001)$ der SUI nach TOT-Operation. Schlussfolgerung: Die Einlage des transobturatorischen Bandes ist eine einfache, sichere und wirkungsvolle chirurgische Methode zur Therapie der weiblichen SUI und ermöglicht zusätzlich eine postoperative Neujustierung. Diese Technik scheint eine attraktive Alternative zu sein, falls sich die bisher erreichten guten Resultate auch im Langzeitverlauf bestätigen.

\section{P III/32 Urogynäkologie \\ Gynecare MoniTorr $^{\circledR}$ : Erste Erfahrungen mit der URP-Messung zur Abklärung der weiblichen Belastungsinkontinenz}

\author{
Schneider Ch. ${ }^{\mathrm{a}}$, Pfander M. ${ }^{\mathrm{b}}$, Fink D. ${ }^{\mathrm{a}}$, Perucchini $D .^{\mathrm{a}}$ \\ ${ }^{a}$ Klinik für Gynäkologie, Departement Frauenheilkunde, \\ Universitätsspital Zürich, ${ }^{\mathrm{b}}$ Gynecare, Spreitenbach
}

Einführung: Bei der Abklärung der Harninkontinenz ist die Urodynamik heute Standard. Dabei werden Ruhe- (MUCP) und Stressprofil gemessen. Die Pathophysiologie der Inkontinenz ist nicht vollständig geklärt. Eine neue Messmethode misst die urethrale Verschlussfunktion über den sogenannten Urethra-Rückstaudruck (urethral retro-resistance pressure, URP). In einer Studie mit 258 Patientinnen konnten Slack et al. [Neurourol Urodyn 2004;23:109-114] eine konsistente Korrelation des URP mit dem 
Inkontinenz-Schweregrad nachweisen. Mit der vorliegenden Arbeit wollten wir dieses Gerät testen und mit der herkömmlichen Messung vergleichen. Material und Methoden: Zwischen November 2004 und Februar 2005 wurden in unserer urogynäkologischen Spezialsprechstunde bei 9 Patientinnen mit Belastungsinkontinenz $(n=5)$ und Mischinkontinenz $(n=4)$ jeweils zusätzlich zur herkömmlichen Urodynamik eine Messung des URP und ein Cystometrogramm $(\mathrm{CMG})$ mit dem MoniTorr ${ }^{\circledR}$ durchgeführt. Ergebnisse: Der durchschnittliche URP aller Patientinnen betrug 59,9 (3182) $\mathrm{cm} \mathrm{H}_{2} \mathrm{O}$, während der durchschnittliche urodynamische Ruhedruck 38,6 (12-90) $\mathrm{cm} \mathrm{H}_{2} \mathrm{O}$ betrug. Mit diesen URP-Werten konnte gemäss Auswertungstabelle die anamnestische Belastungsinkontinenz bei allen Patientinnen bestätigt werden. Eine mögliche Korrelation zwischen URP und urodynamischer Messung kann aus diesen wenigen Zahlen nicht abgelesen werden. Zusätzlich konnten bei Patientinnen mit Detrusorkontraktionen diese mit dem CMG nachgewiesen werden. Schlussfolgerung: Das MoniTorr $^{\circledR}$ ist ein einfach zu bedienendes Gerät, mit dem eine Belastungsinkontinenz nachgewiesen werden kann. Ebenso können Detrusorkontraktionen aufgezeichnet werden. Auf jeden Fall bedarf es auch mit diesem Gerät zur Interpretation der Befunde fundierte urogynäkologische Kenntnisse. In der Literatur ist der URP als Messparameter der urethralen Funktion noch umstritten.

\section{P III/33 Urogynäkologie \\ Umfrage zu den Inkontinenzoperationen in der Facharzt-Ausbildung}

Hallenbarter C., Scheiner D., Perucchini D., Fink D.

Klinik für Gynäkologie, Departement Frauenheilkunde, Universitätsspital Zürich

Einführung: Ab Juli 2005 werden für den schweizerischen Facharzt in Gynäkologie und Geburtshilfe neu zehn Inkontinenzoperationen verlangt, was sich vom deutschen und österreichischen Anforderungskatalog unterscheidet. Diese Situation führt zum Dilemma zwischen einer qualitativ hochstehenden Assistentenweiterbildung und dem Therapieerfolg, der sich unmittelbar an der Patientinnenzufriedenheit messen lässt. Es wurde, im Hinblick auf die Erweiterung des Facharztkataloges, eine schweizweite Umfrage bei allen Chefärzten/Innen für Gynäkologie und Geburtshilfe zum Thema Inkontinenzoperationen an Weiterbildungskliniken durchgeführt. Methode: Es wurde ein anonymisierter Umfragebogen an alle 74 Klinikleiter geschickt, um die Zahl der Inkontinenzoperationen im Allgemeinen und der Schlingenoperationen im Speziellen zu erfassen. Ebenfalls wurde die Anzahl der Operateure und ihre persönliche Meinung zur Einführung der Inkontinenzoperationen erhoben. Resultate: 51 Klinikleiter haben den Fragebogen vollständig ausgefüllt retourniert (69\%). 43 von 49 Chefärzten haben die an ihrer Klinik im Jahr 2004 durchgeführten Inkontinenzoperationen quantifiziert. Bei nahezu 2000 Eingriffen wurde in 50\% ein TVT (tension-free vaginal tape), in 38\% ein TOT (transobturator tape), in 10\% eine laparoskopische, vaginale oder abdominale Kolposuspension und in 2\% eine andere Inkontinenzoperation durchgeführt. Bis anhin dürfen Inkontinenzoperationen aber erst in 7 von 51 Kliniken (14\%) durch Assistenzärzte vorgenommen werden. Auf die Frage, welche der Inkontinenzoperationen als «Einsteiger-Operation» geeignet sei, erachten 57\% der Klinikleiter keine der Inkontinenzoperationen, 17\% das TVT und 15\% das TOT als hierzu geeignet. 25 Chefärzte $(50 \%)$ befürworten die Neuaufnahme der Inkontinenzoperationen in den Facharztkatalog. Verschiedentlich wurde als Kommentar die Trennung von operativem und nicht-operativem Facharzt gefordert, wobei der Nach- weis von Inkontinenzoperationen im operativen Facharztausweis erwünscht wäre. Schlussfolgerung: Bei ungefähr 50 Facharztabsolventen pro Jahr können zehn Inkontinenzoperationen pro Bewerber gewährleistet werden. Es besteht bei den Ausbildnern Einigkeit, dass Inkontinenzoperationen zu den anspruchsvollen Eingriffen zählen, was sich in der eher tiefen Anzahl Kliniken zeigt, wo Assistenzärzte bis anhin solche Eingriffe vornehmen durften. Eine urodynamische Grundausbildung und anatomisches Verständnis für den Beckenboden werden vor Durchführen von Inkontinenzoperationen vorausgesetzt.

\section{P III/34 Urogynäkologie \\ Unterschiedliche Lebensqualität bei Patientinnen mit Stress- und Urgeinkontinenz}

Rubenov R., Scheiner D., Perucchini D., Fink D.

Klinik für Gynäkologie, Departement Frauenheilkunde, Universitätsspital Zürich

Einführung: Fragebogen zur Einschätzung der Lebensqualität werden zur Beurteilung des Leidensdruckes bei Patientinnen mit Harninkontinenz immer wichtiger. Der King's Health Questionnaire (KHQ) besteht aus 9 Fragegruppen (z.B. körperliche, soziale Einschränkungen) und ist für die überaktive Blase (OAB) und Belastungsinkontinenz (SUI) validiert. Seit Juni 2002 setzen wir diesen Fragebogen bei unseren Patientinnen mit Inkontinenz ein. Wir prüften die Nullhypothese, dass sich die Lebensqualität der Patientinnen mit SUI, OAB und Mischinkontinenz (Mix) nicht unterscheidet. Methodik: Zwischen Juni 2002 und Dezember 2004 führten wir bei 663 Patientinnen eine urogynäkologische Untersuchung durch. Im KHQ erhoben wir aus den 9 Fragegruppen 9 Scores und werteten diese für Patientinnen mit SUI, OAB und Mix aus. Wir prüften den Einfluss von Alter und ausgewählter urodynamischer Parameter auf die Einschränkung der Lebensqualität. Die statistische Auswertung erfolgte mittels logistischer Regression und dem Kruskal-Wallis-Test. Ergebnisse: 373 Fragebogen konnten ausgewertet werden. 98 Frauen $(30,3 \%)$ hatten eine SUI, 71 $(21,9 \%)$ eine OAB und $155(47,8 \%)$ eine Mix. Das Durchschnittsalter betrug 58,5 Jahre (SD 14,5, 19,7 bis 91 Jahre) und unterschied sich nicht signifikant für die Gruppen SUI, Mix und OAB. Der Schweregrad der Einschränkung der Lebensqualität bei Patientinnen mit SUI, OAB und Mix unterschied sich signifikant bis auf die Teilscores «Incontinence Impact», «Physical Limitations» und «Emotions», wobei Patientinnen mit Mix die grösste Beeinträchtigung aufwiesen, gefolgt von Patientinnen mit OAB. Ein früher erster Harndrang korrelierte mit einer schlechteren Lebensqualität. Alter und Urethralruhedruck beeinflussten den Score nicht. Schlussfolgerung: Es gilt, bei der Therapieplanung speziell die grössere Beeinträchtigung von Patientinnen mit gemischter Inkontinenz zu berücksichtigen.

\section{P III/35 Urogynäkologie \\ Urodynamische Praxis in der Schweiz: Wie ist der aktuelle Stand?}

Treustedt J. ${ }^{\mathrm{a}}$, Spinosa J.P. ${ }^{\mathrm{b}}$, Kuhn A. ${ }^{\mathrm{a}}$

aFrauenklinik, Inselspital Bern,

${ }^{\mathrm{b}}$ Gynäkologische Privatpraxis, Lausanne

Einführung: Urodynamische Untersuchungen werden von Gynäkologen und Urologen durchgeführt - aus klinischen und foren- 
sischen Gründen, zur Diagnosestellung und vor Inkontinenzoperationen. Zum tatsächlichen Stand der urodynamischen Praxis in der Schweiz existieren keine Daten. Es ist unklar, welche Untersuchungen als «Urodynamik» erachtet werden, welche von den Untersuchern als sinnvoll befunden und welche praktisch durchgeführt werden. Material und Methoden: Es wurden alle Fachärzte für Gynäkologie und Urologie in der Schweiz ermittelt und gebeten, einen Fragebogen auszufüllen. Hauptthemen dieses Fragebogens waren die Indikationen für die Urodynamik, eine Spezifizierung der angewandten urethralen und zystometrischen Funktionstests sowie die Beurteilung derselben und die jährliche Anzahl pro Untersucher. Ergebnisse: Von 1247 versandten Fragebögen wurden 233 zurückgesandt (19\%) - aus folgenden Bereichen (Deutschschweiz vs. Romandie): Privatpraxis/-klinik (66 vs. 61\%), Regionalspital (19 vs. $23 \%$ ), Regionalspital kombiniert mit Privatpraxis (10 vs. $10 \%$ ), Universitätsspital (3 vs. 4\%), Universitätsspital kombiniert mit Privatpraxis (2 vs. 2\%). Dies entspricht der Verteilung unter den angeschriebenen Fachärzten. Diese 233 Ärzte behandeln jährlich ingesamt 5418 Patienten, 90 von ihnen führen selber urodynamische Messungen durch. Als wichtigste Indikationen für diese Untersuchungen werden eine neu aufgetretene Inkontinenz, unklare Symptome, neurologische Probleme und die präoperative Abklärung gesehen. Folgende diagnostische Hilfen werden von den befragten Ärzten als sehr wichtig eingestuft (Deutschschweiz vs. Romandie): Miktionskalender (71 vs. 21\%), Hustentest (77 vs. $50 \%$ ), Q-tip test (14 vs. 14\%). Als wichtigste Tests werden die Restharnbestimmung (62\%) und das Urethrastressprofil (62\%) angesehen. Der Einfluss auf die anschliessende Therapie wird in der Deutschschweiz (30\% «fast immer») grösser eingeschätzt als im französischsprachigen Landesteil (12\% «fast immer»). Schlussfolgerung: Urodynamische Untersuchungen werden von einem grossen Teil der Gynäkologen und Urologen als wichtig eingestuft. Es bestehen unterschiedliche Meinungen dazu, wann, zu welchem Zweck und welche Untersuchungen genau durchgeführt werden sollen.

Mit freundlicher Unterstützung von Gynecare Switzerland

\section{P III/36 Urogynäkologie \\ Verändertes Miktionsverhalten nach TVT-Operation}

\section{Berner F., Scheiner D., Perucchini D., Fink D.}

Klinik für Gynäkologie, Departement Frauenheilkunde, Universitätsspital Zürich

Einführung: Gelegentlich beschreiben Patientinnen nach TVTEinlage eine länger dauernde Miktion als vor der Operation oder schildern, dass sie die Toilette bei Harndrang rascher aufsuchen müssen. Ziel dieser Arbeit war es, diese Aussagen zu objektivieren. Methodik: Bei 49 Patientinnen mit Belastungs- oder gemischter Inkontinenz führten wir eine standardisierte urogynäkologische Untersuchung mit Miktiometrie vor und nach Bandeinlage durch. Zudem führten die Patientinnen einen Miktionskalender. Wir verglichen die prä- und postoperativen Werte der Miktiometrie (erster Harndrang, maximale Blasenkapazität, maximale Flussrate, Miktionszeit) und vom Miktionskalender (Miktionsfrequenz und Miktionsmenge) und analysierten diese in Abhängigkeit einer normotonen und hypotonen (maximaler Urethralruheverschlussdruck $20 \mathrm{~cm} \mathrm{H}_{2} \mathrm{O}$ ) Urethra. Die statistische Auswertung erfolgte mittels t-Test, Wilcoxon- resp. Mann-Whitney-Test. Ergebnisse: Das Durchschnittsalter betrug 66,1 Jahre (SD 9,0; 43,8-79,7), wobei die 21 Frauen mit hypotoner Urethra signifikant älter waren als die 28 mit normotoner $(71,0$ resp. $62,5, \mathrm{p}<0.05) .19$ Frauen $(38,8 \%)$ wiesen eine reine, $30(61,2 \%)$ eine gemischte Inkontinenz auf. Post- operativ waren $89,3 \%$ der Frauen mit normotoner und 73,7\% mit hypotoner Urethra kontinent (negativer Hustentest). Die Miktionszeit nahm signifikant von 41 auf $55 \mathrm{~s} \mathrm{zu}(\mathrm{p}=0,004)$ resp. die maximale Flussrate von 35 auf $26 \mathrm{ml} / \mathrm{s}$ ab $(\mathrm{p}=0,04)$. Die Miktionsmenge nahm von 219 auf $256 \mathrm{ml} z u(p=0,03)$, während die Miktionsfrequenz konstant blieb $(8,5$ resp. 7,7, p =0,15). Bei hypotoner Urethra waren prä- und postoperativ erster Harndrang sowie postoperativ Blasenkapazität und Flussrate signifikant im Vergleich zur normotonen Urethra erniedrigt. Schlussfolgerung: Die urodynamisch nachgewiesene Reduktion der maximalen Flussrate sowie die verlängerte Miktionszeit nach TVT-Einlage objektiviert das gelegentlich als verändert geschilderte Miktionsverhalten. Das TVT kann obstruktiv wirken. Nach Auswertung des Miktionskalenders vermuten wir aufgrund der postoperativ höheren Miktionsmenge bei gleichbleibender Miktionsfrequenz, dass die Patientinnen länger mit der Miktion zuwarten und dann einen umso stärkeren Harndrang verspüren. Die Patientinnen sollen präoperativ entsprechend aufgeklärt werden.

\section{P III/37 Urogynäkologie 50 TVT-O-Inkontinenzoperationen, ein Vergleich}

Gaudenz R., Dellas A., Mariotta M., Geiger S.

Frauenklinik, Kantonsspital Liestal

Einführung: An der Frauenklinik Liestal wurde von der konventionellen TVT-Technik von Gynecare (Johnson \& Johnson) vollständig auf die TVT-O-Version umgestellt (TVT-O: Tension Free Vaginal Tape - Obturator). Es handelt sich dabei um eine «inside-out-Technik», d.h. die Nadelführung geht von innen (parasuburethral) nach aussen (durch die Membrana obturatoria) zum Oberschenkel, was viele Vorteile bietet und uns voll überzeugt. Material und Methode: Die ersten 50 Fälle mit der neuen Technik werden vorgestellt und mit 50 konventionellen Fällen von TVT verglichen. Ergebnisse: Die TVT-O-Technik (Gynecare) ist einfacher, schneller und sicherer als die konventionellen TVT-Technik von Prof. Ulf Ulmsten (Gynecare). Der Aufwand mit dem TVTO-System ist kleiner. Die Ergebnisse sind, soweit bis heute beurteilbar, mit den Ergebnissen der konventionellen TVT-Technik absolut vergleichbar. Blasenperforationen und andere Verletzungen kommen nicht vor, denn die Nadel tangiert den Innenraum des kleinen Beckens an keiner Stelle. Blutungen aus Spatium-retii-Gefässen kommen nicht vor. Die Zystoskopien entfallen. Es ist vorteilhaft, die TVT-O-Operation in Spinalanästhesie zu machen. Die Anwendung der Lokalanästhesie mit Analgosedierung ist noch eine zu prüfende Option. Schlussfolgerung: Die TVT-O-Inkontinenzoperation von Gynecare, die von Prof. Jean de Leval in Liège, Belgien in hervorragend durchdachter Weise entwickelt wurde, ist Anwender-freundlich, sicher und garantiert bei korrekter Anwendung sehr gute Resultate.

\section{P III/38 Urogynäkologie ICSplus: Eine Erweiterung des Prolapsstagings der hinteren Vaginalwand}

Najjari L. ${ }^{a}$, Kuschel S. ${ }^{a}$, Werner M. ${ }^{\mathrm{b}}$, Schüssler S. ${ }^{\mathrm{a}}$, Bucher $S{ }^{\mathrm{a}}$

${ }^{a}$ Neue Frauenklinik, Kantonsspital Luzern, ${ }^{b}$ Frauenklinik, Universitätsspital Zürich

Einleitung: Bezüglich des vaginalen Prolapsstagings bedienen sich Gynäkologen international des standardisierten Systems der
Abstracts, gynécologie suisse, Lugano, 2005 
International Continence Society (ICS). Die Bestimmung des maximalen Ausmasses des Prolaps erfolgt beim Pressen. Auf die hintere Vaginalwand (hVW) bezogen sind jedoch abhängig vom Füllungszustand der rektalen Ampulle Unterschiede im Ausmass des Prolaps der hVW denkbar, die im Zustand des Pressens allein nicht erfasst werden. Ziel dieser Studie war es, den Prolaps der hWV unter digital rektaler Mobilisierung zu messen, nach dem herkömmlichen ICS-Prolapsstaging zu klassifizieren und mit diesem zu vergleichen. Material und Methode: 59 Patientinnen nahmen an der prospektiven Studie teil. Neben dem herkömmlichen ICS-Prolapsstaging wurde das Ausmass der digital rektalen Mobilisierbarkeit der hVW metrisch in einem dreidimensionalen System dokumentiert: (a) stellte die Mobilisierbarkeit der hWV nach cranial, (b) die Mobilisierbarkeit zum Untersucher hin und (c) die Mobilisierbarkeit nach lateral dar. Danach wurde die mobilisierte hVW nach dem ICS-System klassifiziert und als ICSplus bezeichnet. Bei 26 Patientinnen wurde zudem die Reproduzierbarkeit von ICSplus durch zwei unabhängige Untersucher überprüft. Ergebnisse: ICSplus zeigte eine gute Reproduzierbarkeit. Die Strecken (a), (b) und (c) betrugen $1,45 \pm 0,87 \mathrm{~cm}(0-3 \mathrm{~cm}), 1,78 \pm 1,06 \mathrm{~cm}(0-5$ $\mathrm{cm})$ und 1,36 $\pm 0,66 \mathrm{~cm}(0-3 \mathrm{~cm})(\mathrm{MW}, \mathrm{SD}$ (range)). Durch digitale rektale Mobilisation der hVW konnte bei $87 \%$ der Patientinnen eine Diskrepanz zwischen ICS und ICSplus-Grad $\geqslant$ I ermittelt werden $(p<0,001)$. Entsprechend der ICS-Klassifikation wurden folgende Häufigkeiten des Ausmasses des Prolaps der hVW ermittelt: Grad 0, I, II, III, IV: 23,7, 15,3, 45,8, 15,3, 0\%, bei der erweiterten ICSplus-Klassifikation hingegen 5,1, 11,9, 37,3, 45,8, 0\%. Schlussfolgerung: Die ICS-Klassifikation unterscheidet sich stat. signifikant von der ICSplus-Klassifikation. ICSplus scheint eine notwendige Erweiterung der ICS-Klassifikation zu sein, da bei den meisten Patientinnen das maximal mögliche Ausmass des Prolaps der hVW durch Pressen alleine nicht erfasst wird.

\section{P IV/40 Geburtshilfe \\ Geburtseinleitung bei intrauterinem Fruchttod mittels einer Kombination von Mifepriston und Prostaglandinen - Eine retrospektive Analyse}

Gairing A., Surbek D., Holzgreve W., Hösli I.

Universitäts-Frauenklinik, Basel

Einleitung: Das Ziel dieser retrospektiven Studie war es, unser Vorgehen zur Geburtseinleitung bei intrauterinem Fruchttod (IUFT) mit einer Kombination von Mifepriston und Misoprostol/ Dinoproston hinsichtlich Effektivität und Sicherheit zu überprüfen. Methoden: Wir werteten retrospektiv Daten aller Patientinnen aus, die zwischen dem 1.1.2002 und dem 31.12.2004 an unserer Klinik mit IUFT ab der 24. SSW betreut wurden. Bis 9/2002 wurde allein mit Prostaglandinen (PG)/Oxytocin behandelt, seit 10/2002 haben wir folgendes neues Behandlungsregime eingeführt: Bei Bishop-Score $\leq 6$ geben wir $200 \mathrm{mg}$ Mifepriston p.o. und idealerweise 24-36 h später Misoprostol vaginal. Die Dosis ist abhängig von der SSW: $100 \mu \mathrm{g}$ alle $6 \mathrm{~h}$ zwischen 24-33 + 0 SSW, ab 33 $+1 \mathrm{SSW} 50 \mu \mathrm{g}$ alle $6 \mathrm{~h}$ (max. $4 \times / 24 \mathrm{~h}$ ). Bei St.n. Sectio applizieren wir Dinoproston $3 \mathrm{mg}$ vaginal alle $6 \mathrm{~h}$ (max. 2 Dosen/24 h). Bei einem Bishop-Score $>6$ erfolgt die Amniotomie und Oxytocin intravenös. Die Auswertung der Daten erfolgte mittels deskriptiver Statistik. Ergebnisse: Eine konsekutive Serie von 18 Frauen wurde betreut, im Mittel 32 Jahre alt (22-41), das Gestationsalter betrug 24 bis $40 \mathrm{SSW}$. Zwei hatten bei Aufnahme spontane Wehentätigkeit. Einmal wurde mit Amniotomie und Oxytocin, bei 6 Frauen mit PG alleine eingeleitet. Bei 8 Frauen kam das neue Regime zum Einsatz, eine Frau entwickelte spontane Wehen nach Mifepriston. Vergleicht man die Gruppe Mifepriston und PG mit der Gruppe PG/Oxytocin allein, war die durchschnittlich benötigte Misoprostoldosis niedriger $(260 / 400 \mu \mathrm{g})$ und die Dauer vom Zeitpunkt der Einleitung mit PG bis zur Geburt kürzer $(15 \mathrm{~h} / 17 \mathrm{~h})$ bei gleichem durchschnittlichen Gestationsalter. Es gab einen Fall von schwerer Atonie mit DIC in der Gruppe Mifepriston/PG, ansonsten zeigte sich kein Unterschied hinsichtlich der Morbidität. Die Wahrscheinlichkeit, innerhalb von $24 \mathrm{~h}$ zu gebären, lag mit der Kombination von Mifepristone und PG bei über $85 \%$, innerhalb von $48 \mathrm{~h}$ über $95 \%$. Schlussfolgerung: Die Kombination von Mifepriston und Misoprostol/Dinoproston ist ein effektives und schonendes Verfahren zur Geburtseinleitung bei IUFT. Mifepriston reduziert die Dosis der PG und verkürzt das Intervall zwischen Priming und Geburt. Auch für das betreuende Team ist die gute zeitliche Steuerbarkeit ein grosser Vorteil.

\section{P IV/41 Geburtshilfe \\ Beckenendlage: Von Bracht zur Sectio}

Csizy-Botond L., Brülhart G., Herrmann U.

Frauenklinik Spitalzentrum, Biel

Einführung: Der Term Breech Trial (TBT) hat zur weltweiten Diskussion über die optimale Geburt aus Beckenendlage (BEL) geführt. Die Studie hat gezeigt, dass bei vaginaler Geburt aus BEL die Risiken für das Kind leicht höher sind als bei einer Sectio caesarea. In den Niederlanden führte diese Studie sehr rasch zu einer Zunahme der Sectiones wegen BEL, die vaginale Steissgeburt nahm von $50 \%$ auf unter $20 \%$ ab. Wir untersuchten, ob in der Schweiz ein ähnliches Phänomen festzustellen und ob für das kindliche Out-come ein Benefit gemessen am pH der Nabelschnurarterie (Asphyxiedefinition: $\mathrm{pH}<7.10$ ) zu erkennen ist. Unsere Klinik hat in Zusammenarbeit mit der Universitätsklinik Genf an der TBT-Studie teilgenommen. Patientinnen und Methode: Die Daten der Arbeitsgemeinschaft der Schweizerischen Frauenkliniken wurden ausgewertet. Die Arbeitsgemeinschaft umfasst 78 öffentliche und 2 private Frauenkliniken. Mit der Statistik werden rund $30 \%$ aller Geburten in der Schweiz erfasst. Vier Jahre vor der Publikation der TBT-Studie wurden mit den 4 Jahren nach 2000 verglichen. Als weiterer Vergleich diente das Jahr 1989, das die Praxis rund 10 Jahre vor der TBT zeigen soll. Ergebnisse: In den Jahren 1997 bis 2000 wurden die meisten Kinder aus BEL durch Sectio caesarea entbunden. Nach dem Jahr 2000 zeigte sich praktisch eine Halbierung der vaginalen Entbindungen aus BEL. Gleichzeitig mit der Zunahme der Sectiones bei BEL fällt eine deutliche Reduzierung der azidotischen Kinder ( $\mathrm{pH}<7.10$ ) um $42 \%$ auf. Schlussfolgerung: Die TBT-Studie hat in der Schweiz vermutlich analog zu den Niederlanden zu einem Rückgang der vaginalen Geburt aus BEL geführt. Im Jahr 2004 wurden gemäss der ASF-Statistik nur noch 59 Kinder aus BEL vaginal nach Bracht entbunden. Unsere Arbeit zeigt, dass gross angelegte Studien (z.B. WHI), die auch in den Medien zitiert werden, zu einem relativ raschen Wandel der Praxis führen. Zunehmendes Sicherheitsdenken der werdenden Eltern wie auch die Angst vor forensischen Folgen hat uns in die Defensive gebracht. Dies kann auch die Zunahme der Gesamtsectiorate bei BEL von 80 auf 90\% erklären. Daten der ASF erlauben uns, kurz- und mittelfristige Trends in der Geburtshilfe und Gynäkologie zu erkennen. Die Kausalität dieser Ergebnisse bedarf jedoch der Interpretation und Diskussion. 


\section{P IV/42 Geburtshilfe \\ Môle hydatiforme: Une exception pour rappeler quelques règles}

Söhnchen N. ${ }^{a}$, Pellegrinelli J.-M. ${ }^{a}$, Duc C. ${ }^{\mathrm{b}}$, Aymon D. ${ }^{\mathrm{a}}$, Schneider $N .^{a}$

aService de Gynécologie et d'Obstétrique, Réseau Santé Valais, Hôpital Régional Martigny, 'bervice de Pathologie, Réseau Santé Valais, Hôpital Régional Sion

But: A partir d'un cas exceptionnel de môle hydatiforme complète diploïde chez une patiente de 53 ans en péri-ménopause, nous faisons une revue rapide de la littérature actuelle et nous définissons la prise en charge adéquate dans une telle situation. Présentation du cas: Il s'agit d'une patiente primigeste, primipare de 53 ans avec un accouchement sans particularité intervenu 32 ans plus tôt, au bénéfice d'une contraception orale jusqu'à l'âge de 51 ans, avec la persistance de cycles réguliers malgré l'apparition d'un syndrome climatérique depuis quelques mois. Après une aménorrhée secondaire de 2 mois accompagnée d'une discrète symptomatologie gravidique, apparition de ménométrorragies évoquant des règles. Une consultation gynécologique met en évidence un utérus agrandi correspondant à 12 semaines d'aménorrhée avec un test de grossesse fortement positif et un contenu suggérant une môle. Le diagnostic est confirmé lors de l'évacuation spontanée du matériel trophoblastique. Conclusions: La maladie gestationnelle trophoblastique (MGT) est très rare à partir de 50 ans mais décrite jusqu'à un âge avancé de 61 ans avec une tendance accentuée vers la malignité. Une lecture attentive de la littérature ne fait pas mention de cas intervenus chez des femmes ménopausées selon les critères de l'Organisation Mondiale de la Santé (absence de règles, sans forme de substitution hormonale, de plus de 12 mois). Le contenu utérin doit être évacué soit par aspiration avant tout traitement utérotonique pour diminuer le risque de dissémination par voie sanguine, soit par hystérectomie totale. Une approche systématique comprenant un bilan d'extension avec staging et scoring, sanctionnée par une consultation de gynécologie oncologique permet de définir la suite du traitement et d'améliorer le pronostic. Les béta-HCG sont le marqueur spécifique de cette tumeur et leur profil doit être suivi jusqu'à négativation confirmée sur 6 mois. La contraception doit être assurée pour un minimum de 12 mois.

\section{P IV/43 Geburtshilfe \\ Normkurve für den Symphysen-Fundus- Abstand bei Zwillingsschwangerschaften}

\author{
Staub $D .^{\mathrm{a}}$, Harpes $P^{\mathrm{b}}$, Zimmermann . $^{\mathrm{a}}$, \\ Ochsenbein-Kölble N. ${ }^{a}$ \\ ${ }^{a}$ Klinik für Geburtshilfe, Universitätsspital Zürich, \\ ${ }^{b}$ Abteilung Biostatistik, Institut für Sozial- und \\ Präventivmedizin, Universität Zürich
}

Einführung: Die Messung des Symphysen-Fundus-Abstandes (SFA) in der Schwangerschaft ist eine weit verbreitete, nicht invasive und billige Methode, das intrauterine Wachstum eines Kindes zu überprüfen. In der Literatur finden sich wenig Informationen zum SFA bei Zwillingsschwangerschaften, weshalb es unser Ziel war, hierfür eine Normkurve zu erstellen. Patienten und Methoden: In dieser Querschnittstudie mit einer Datenerhebung über einen Zeitraum von 10 Jahren wurden 257 schwangere Zwillingsmütter eingeschlossen. Der SFA wurde entsprechend der Methode von Westin gemessen. Schwangere mit einem IUFT oder einer schweren fetalen Fehlbildung, Drogen- oder Alkoholabusus, schweren Nierenerkrankungen, einem Uterusmyom $>5 \mathrm{~cm}$ oder einer Uterusfehlbildung, die den SFA beeinflusst, wurden ausgeschlossen. Ergebnisse: Der mittlere SFA lag in der 20., 25. und 30. SSW bei 23, 29 und $34 \mathrm{~cm}$ und stieg bis zur 37 . SSW auf $42 \mathrm{~cm}$ an. SFAWerte von $<17 \mathrm{~cm}$ in der 20. SSW, $<23 \mathrm{~cm}$ in der 25 . SSW, $<28 \mathrm{~cm}$ in der 30. SSW und $<35 \mathrm{~cm}$ in der 37. SSW lagen unter der 5. Perzentile. Schlussfolgerung: Mit unserer Studie war es möglich, Normkurven für der SFA bei Zwillingsschwangerschaften zu erstellen. Es bleibt noch zu evaluieren, ob mit diesen Normkurven Zwillingspärchen, bei denen beide Kinder Small for Gestational Age (SGA) sind, erkannt worden wären.

\section{P IV/44 Geburtshilfe \\ Vorzeitiger Blasensprung vor der \\ 35. Schwangerschaftswoche: Schwangerschafts- und neonatales Outcome}

Birkenmaier A. ${ }^{a}$, Fontana M. ${ }^{\mathrm{b}}$, Zanetti R. ${ }^{\mathrm{a}}$, Schulzke S. ${ }^{\mathrm{b}}$, Holzgreve W. ${ }^{a}$, Hösli I. ${ }^{a}$

aUniversitäts-Frauenklinik Basel,

bUniversitäts-Kinderspital beider Basel

Einführung: In dieser retrospektiven Studie wird der Einfluss schwangerschaftsverlängernder und begleitender Massnahmen (Lungenreifungsinduktion, Tokolyse und Antibiotika) bei vorzeitigem Blasensprung auf das neonatale Outcome untersucht. Methoden: Erfasst wurden Frauen mit vorzeitigem Blasensprung (preterm premature rupture of membranes; $\mathrm{pPROM}$ ) vor der $33+6$ SSW und Geburt nach der $24+0$ SSW. Ab $34+0$ SSW u/o Auftreten von maternalen Infektzeichen, Kontraktionen, vaginalen Blutungen oder suspektem CTG wurde eine Sektio durchgeführt oder die Geburt eingeleitet. Die neonatale Morbidität wurde durch das Vorhandensein einer Sepsis, Infant Respiratory Distress Syndrom (RDS), nekrotisierenden Enterokolitis (NEC), intrakraniellen Blutung (ICB) und der Aufenthaltsdauer auf der Intensivstation erfasst. Von 2000 bis 2004 wurden 72 Frauen und 85 Kinder eingeschlossen. Eine Patientin mit intrauterinem Fruchtod wurde ausgeschlossen. Ergebnisse: Entsprechend der Latenz zwischen Blasensprung und Geburt wurden 3 Gruppen gebildet: $A$ ( $n=20$, Geburt $<48 \mathrm{~h}), \mathrm{B}(\mathrm{n}=26$, Geburt $<7 \mathrm{~d})$ und $\mathrm{C}(\mathrm{n}=25$, Geburt $>7 \mathrm{~d})$. Das Gestationsalter bei Blasensprung war in A und $\mathrm{B}$ höher als in C (31,4 vs. 31,8 vs. 27,$1 ; p=0,001$ bzw. $p<0,001)$. Die Zervixlänge bei pPROM war in A kürzer als in B und $C(p=0,021$ bzw. $p=$ 0,027 ). Die Häufigkeit einer Chorioamnionitis unterschieden sich nicht (63 vs. 44 und 57\%; $p=0,736$ ), obwohl maternale Infektzeichen häufiger in $\mathrm{C}$ auftraten (50 vs. 54 und 84\%; $\mathrm{p}=0,021$ ). Kein Unterschied für: Geburtsgewicht, positiven Vaginalabstrich oder vorzeitige Plazentalösung. Frühgeborene aus A litten häufiger unter ICB als in B und in C (21 vs. 0 vs. 5\%; $\mathrm{p}=0,050)$. Für RDS, NEC und Intensivaufenthalt ergaben sich keine signifikanten Unterschiede. Tendenziell kam es in C häufiger zu Septitiden (13 vs. 20 vs. $39 \% ; p=0,099)$. Die perinatale Mortalität unterschied sich nicht (1 Todesfall in A und C; $p=0,673$ ). Schlussfolgerungen: Unter engmaschiger maternaler und fetaler Kontrolle ist ein konservatives Management bei pPROM gerechtfertigt. Neben der resistenzgerechten Antibiotikagabe und Lungenreifung scheint unseren Ergebnissen nach bei weiterhin fehlenden Hinweisen für eine Chorioamnionitis eine Tokolyse über $48 \mathrm{~h}$ hinaus sinnvoll.
Abstracts, gynécologie suisse, Lugano, 2005 


\section{P IV/45 Geburtshilfe \\ Hyperreactio luteinalis: Rein konservatives Management eines Adnextumors im Oberbauch}

Bütikofer K., Stierli M., Kleeb B., Beck G.

Frauenklinik, Kantonsspital Olten

Einleitung: Die Hyperreactio luteinalis ist eine selten auftretende Begleiterkrankung der Schwangerschaft. Charakteristisch ist eine bilaterale (selten unilaterale) Vergrösserung der Ovarien (durch Theca lutein Zysten) und ein erhöhter Beta-hCG-Spiegel. Das klinische Bild als auch die pathophysiologischen Veränderungen sind ähnlich dem ovariellen Überstimulationssyndrom(OHSS). Fallbericht: 32-jährige GII PI in der 8 6/7 Schwangerschaftswoche, präsentiert sich mit Unterbauchschmerzen beidseits. Mässige Emesis. Sonst unauffälliger Schwangerschaftsverlauf. Gynäkologische Anamnese: Zyklen regelmässig alle 30 Tage, Hypermenorrhoe, St.n. Sectio wegen BEL 5/02. Antikonzeption mit Mirena-IUD bis 4/04, danach schnell aufgetretene, spontane Schwangerschaft. Sonographie: Intakte intrauterine Einlingsgravidität entsprechend Amenorrhoe mit sonomorphologisch unauffälliger Plazenta, beide Ovarien massiv vergrössert mit polyzystischen Veränderungen (rechts $95 \times 56 \mathrm{~mm}$; links $90 \times 48 \mathrm{~mm}$ ), mässig Aszitesbildung. Abdomen: Druckdolenz über der rechten Adnexloge sowie über dem linken Ovar, welches gut palpabel unterhalb des linken Rippenbogens liegt. $\mathrm{Hb}$, Hkt, Elektrolyte, Gerinnungsparameter sowie Schilddrüsenwerte im Normbereich, Beta-hCG: 216135 U/l. Unter stationären Bedingungen erfolgen Analgesie, Rehydrierung und Thromboseprophylaxe. Initial Zunahme der Schmerzen und des Aszites. Eine Laparoskopie kann mit dieser konservativen Therapie dennoch vermieden werden, da sich die Symptome langsam bessern. Entlassung der Schwangeren nach 2 Wochen in ordentlichem AZ. Die Nachkontrollen zeigen einen weiterhin günstigen Verlauf mit Abfallen der Beta-hCG-Werte auf die Norm. Die Ovarien bleiben nach wie vor vergrössert. Diskussion: Dieses Krankheitsbild ist meistens mit einer Mehrlingsgravidität, einem Syndrom der polyzystischen Ovarien oder einer Hypothyreose verbunden. Beta-hCG produzierende Tumoren des Trophoblasten oder der Ovarien müssen ausgeschlossen werden. Die klinische Symptome können einem OHSS ähnlich sein. Dementsprechend ist eine konservative Therapie anzustreben (Analgesie, Rehydrierung, Thromboseprophylaxe sowie engmaschige Überwachung). Bei maternalen Komplikationen kann eine operative Therapie, im Extremfall die Beendigung der Schwangerschaft indiziert sein.

\section{P IV/46 Geburtshilfe \\ Morbidità respiratoria acuta successiva al cesario elettivo nei neonati prossimi al termine}

De Luca R. ${ }^{\mathrm{a}}$, Boulvain M. ${ }^{\mathrm{b}}$, Berner M. ${ }^{\mathrm{a}}$, Irion O. ${ }^{\mathrm{b}}$, Morales M.A. ${ }^{\mathrm{b}}$, Pfister R.E. ${ }^{\mathrm{a}}$

aNeonatologia, ${ }^{b}$ Ginecologia e Ostetricia,

Ospedale Universitario di Ginevra

Introduzione: Il cesario (CS) eseguito prima dell'inizio del travaglio o CS elettivo, contribuisce notevolmente all' aumento costante del tasso complessivo dei CS. Tale modalità di parto può talora salvare delle vite, ma uno scarso niumero di studi é disponibile sull'eventuali complicanze neonatali. Lo scopo del presente studio é di analizzare la morbidità neonatale acuta (MNA) successiva al CS elettivo e paragonare i risultati con la MNA osservata nei nati da CS non elettivo e da parto vaginale (VD) dopo 34 settimane di gestazione (SG). Materiali e metodi: Gli archivi elettronici neonatali e materni dell'Ospedale Universitario di Ginevra dal 1981-2004 sono stati uniformati, ripuliti elettronicamente e manualmente, ed infine le informazioni in essi contenute sono confluite in una singola coorte di 62000 nascite. I neonati dopo 34 SG con malformazioni gravi sono stati esclusi dall'analisi. L'ospedalizzazione è stato il criterio scelto per stabilire la presenza di MNA. Risultati: Tra i 58539 parti dopo le $34 \mathrm{SG}$, sono stati eseguiti 7813 $(13,4 \%)$ CS, $39,7 \%$ prima (BOL) e $60,3 \%$ dopo il travaglio di parto (AOL). La stratificazione per età gestazionale mostra una tendenza verso una riduzione della MNA tra 34 e 38 SG (da 67 a 16,9\%), ed una lieve riduzione a partire dalla quarantesima SG $(28,5 \%)$. Tale tendenza appare analoga in tutti i gruppi, indipendentemente dalla modalità di parto. Paragonando BOL e AOL, il rischio di MNA è superiore nei nati da CS BOL rispetto ai CS AOL tra 34 e 35 SG ed ancora maggiore rispetto ai VD (RR 1.34 CI 1,08-1,67; RR 3,98 CI 3,18-4,98 rispettivamente). Oltre le $36 \mathrm{SG}$, la differenza tra i gruppi si riduce; tra 38 e $39 \mathrm{SG}$, il rischio di MNA nei AOL e BOL non appare statisticamente significativo (RR 1,28 CI 0,96-1,74); rispetto ai $\mathrm{VD}$, l'incidenza di MNA nei BOL è significativamente maggiore tra 38 e 39 SG (RR approssimativamente 2). Conclusioni: Tra 34 e $35 \mathrm{SG}, \mathrm{MNA}$ è superiore nei nati da CS BOL rispetto ai nati AOL e ai VD. Dopo 36 SG, la differenza di MNA tra i gruppi si riduce con il diminuire dell'età gestazionale. Per il CS elettivo il rischio minore è tra 38 e $39 \mathrm{SG}$. La presenza di un pediatra è pertanto auspicabile anche per i neonati prossimi al termine.

\section{P IV/47 Geburtshilfe \\ Bedeutung des CRP-Wertes in der postpartalen Phase}

Antùnez L., Bloch A., Parziale L., Lopez R., Seydoux J.

Département de Gynécologie et d'Obstétrique, Hôpital du Jura, Delémont

Einführung: Die Bestimmung des CRP-Wertes (C-reaktives Protein) ist eine häufig durchgeführte Analyse zur Diagnose und Verlaufsbeurteilung eines infektiösen oder entzündlichen Prozesses. Nach operativen Eingriffen ist der Wert bekannterweise erhöht. Mit unserer Studie wollten wir untersuchen, ob auch die Geburt per se zu einer Erhöhung des CRP-Wertes führt. Material und Methoden: Mit der an unserer Klinik zur Hämoglobinbestimmung routinemässig durchgeführten Blutentnahme am 1. bis 4. postpartalen Tag wurde ebenfalls der CRP-Wert mitbestimmt. Resultate: Der Mittelwert des CRP-Wertes lag bei vaginalen Geburten bei 40,2 mg/l, war somit signifikant erhöht. Tendenz zur einer CRPErhöhung stellten wir ebenfalls bei folgenden Situationen fest, ohne jedoch eine Signifikanz zeigen zu können: Sectio caesarea, protrahierter Geburtsverlauf, Methergineinsatz in der postpartalen Phase, Tag der Blutentnahme (Maximum am zweiten Tag), Grad der Verletzung des Geburtskanales. Keinen Einfluss hatten die Parität, der Antibiotikaeinsatz sub partu und der postpartal bestimmte Hämoglobinwert. Schlussfolgerungen: Der CRP-Wert ist nach jeder Geburt erhöht, sogar auch nach völlig unkomplizierten, physiologischen Spontangeburten. Dieser Tatsache muss unbedingt Rechnung getragen werden, zumal in der postpartalen Periode häufig febrile und subfebrile Zustände auftreten (Subinvolutio uteri, Endometritis, Ovarialvenenthrombosen, tiefe Beinvenenthrombosen, Phlebitiden, Mastitis, Brustwarzenrhagaden, Milchstau) und der CRP-Wert gerne als Entscheidungshilfe zur Ergreifung therapeutischer Massnahmen herangezogen wird. 


\section{P IV/48 Geburtshilfe \\ Antikoagulation bei künstlicher \\ Herzklappe - Diskussion der Guidelines anhand eines Fallbeispieles}

Ladewig A. ${ }^{\mathrm{a}}$, Montavon $\mathrm{C}^{\mathrm{a}}$, Struben $\mathrm{H}^{\mathrm{a}}$, Hetzel . $^{\mathrm{b}}$,

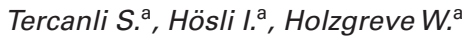

aUniversitäts-Frauenklinik Basel,

bUniversitäts-Kinderspital Basel

Einführung: Eine Schwangerschaft nach Implantierung einer künstlichen Herzklappe birgt ein bedeutsames Gesundheitsrisiko. Besonders die Thrombosierung der Klappe stellt ein relevantes Risiko dar, welches jedoch durch die Wahl der Antikoagulation (AK) beeinflusst werden kann. Die verschiedenen Möglichkeiten der AK, ihre Erfolgschancen und Risiken (inkl. fetale Risiken) werden besprochen. Kasuistik: 28j. IG 0P mit Kinderwunsch. St.n.iv-Drogenabusus mit Endokarditis und nachfolgend $2 \times$ Mitralklappenersatz (97/03). Postoperativ Empfehlung zur perikonzeptionellen Umstellung auf adaptiert dosiertes niedermolekulares Heparin (LMWH) bei AK mit Marcoumar. Narbenepilepsie (Lamotrigin). Behandlung mit Marcoumar bis $24+1$ SSW, dann Umstellung auf LMWH wegen vaginaler Blutung. Sonogr. prominenter fetaler Lateralventrikel DD: Blutung. I Sectio bei $25+4 \mathrm{SSW}$ bei patholog. CTG und vaginaler Blutung bei grossem Randsinushämatom. V.a. Coumarin-Embryopathie. Diskussion: Die Empfehlungen zur optimalen AK sind kontrovers. Die orale AK mit Vit.K-Antagonisten bewirkt nachgewiesenermassen eine sichere und effiziente Antikoagulation für die schwangere Frau. Wesentlicher Nachteil ist die Teratogenität und Beeinträchtigung der ZNS-Entwicklung beim Kind. Unfraktioniertes Heparin (UFH) birgt zwar kein Risiko für den Embryo/Fetus, da nicht plazentagängig, ist aber auch bei dosisadaptiertem Einsatz ungenügend in der Prävention thromboembolischer Komplikationen. Dosis-adaptierte LMWH erscheinen interessant als Alternative, da sie stabiler und besser steuerbar sind, jedoch wenig erforscht und für diese Indikation nicht zugelassen. Ein Mittelweg stellt die Gabe von LMWH bis zur 13. SSW dar, mit anschliessender Umstellung auf orale AK (Warfarin). Schlussfolgerung: Bezüglich der Therapieempfehlungen - insbesondere im 1. Trimenon - besteht kein Konsens. Gegen eine Therapie im 1. Trimenon mit LMWH spricht die ungenügende Datenlage. Allerdings ist denkbar, dass durch den stabileren Antikoagulationseffekt der LMWH gegenüber der UFH die thromboembolischen Komplikationen gesenkt werden können. Diverse Guidelines werden diskutiert. Die Therapieentscheidung muss in Absprache mit der Patientin und den Spezialisten (Kardiologen, Gerinnungsphysiologen und Geburtshelfern) erfolgen.

\section{P V/50 Senologie \\ Mammatumor: Metastase eines Ovarialkarzinoms}

Bertschinger $M .^{\mathrm{a}}$, Bannwart $F^{\mathrm{b}}$, Bürki . $^{\mathrm{a}}$

ârauenklinik Spital Limmattal, 'bPathologie Stadtspital Triemli, Zürich

Einführung: Das Mammakarzinom ist der häufigste maligne Tumor bei Frauen. Mammametastasen von ausserhalb der Brust gelegenen Primärtumoren sind selten. Material und Methoden: Fall-Beschreibung einer Patientin mit ausgedehnten Mammametastasen eines Ovarialkarzinoms und Zusammenfassung der Literatur. Ergebnisse: Bei der 75j. Patientin mit seit beinahe 9 Jahren bekanntem Ovarialkarzinom wurde eine knotige Durchsetzung der rechten Brust und eine axilläre Lympadenopathie festgestellt. Bei dringendem Verdacht auf ein Mammakarzinom wurde eine Feinnadelpunktion durchgeführt, die Verdacht auf eine Metastase eines serös-papillären Ovarialkarzinoms ergab, was mit einer Stanzbiopsie bestätigt wurde. Bei der Patientin wurde 1996 ein serös-papilläres Ovarial-Carcinom Stadium IIIb diagnostiziert. Es wurde eine Chemotherapie (CT) mit 6 Zyklen Carboplatin/Endoxan durchgeführt im Anschluss an eine abdominale Hysterektomie mit Adnexektomie beidseits, pelviner Lymphonodektomie, Omentektomie und second-look Laparotomie mit miliarer Aussaat von Peritonealmetastasen. Nach 28 Monaten Auftreten des ersten Rezidives mit Ascites und Revisionslaparotomie mit Resektion eines stenosierenden Dünndarmabschnittes. In der Folge Durchführung einer CT mit 6 Zyklen Carboplatin/Taxol. Bis zur Diagnose der Mammametastase nach 55 Monaten wurden drei Rezidive erfolgreich mit CT mit Alkeran behandelt. Je nach Quelle handelt es sich bei 0,43-ca. 2\% aller malignen Tumoren der Brust um Metastasen. Es werden Metastasen ausgehend von den unterschiedlichsten Primärtumoren beschrieben. Eine Metastasierung in die Brust tritt bei fortgeschrittener Tumorerkrankung auf, mit schlechter Prognose und einer mittleren Überlebenszeit von wenigen Monaten. Schlussfolgerung: Mammametastasen eines Primärtumors mit Lokalisation ausserhalb der Brust sind selten. Aufgrund der Bedeutung für Therapie und Prognose ist die korrekte Diagnose von grosser Wichtigkeit. Bereits bei atypischem Mammographiebefund oder bei unklarem Histologieergebnis muss eine Metastasierung eines anderen Primärtumors in Erwägung gezogen werden. Dadurch kann eine unnötige radikale chirurgische Therapie, ev. Mastektomie verhindert werden.

\section{P V/51 Senologie \\ Das Schwannom als eine besondere Differenzialdiagnose des palpablen Mammaknotens - Ein Fallbeispiel}

\author{
Schmid N. ${ }^{a}$, Beutler G. ${ }^{a}$, Yeginsoy S. ${ }^{\mathrm{b}}$, Burger D.A. ${ }^{\mathrm{a}}$
}

${ }^{\mathrm{a}}$ Frauenklinik, Spital Lachen, ${ }^{\mathrm{b}}$ Institut für Pathologie, StadtspitalTriemli, Zürich

Einführung: Das Ziel der Abklärungen eines palpablen Mammaknotens ist es, eine zuverlässige Diagnose mit einem Minimum an diagnostischem Aufwand zu stellen. Zur Abklärung gehören die klinische Untersuchung, eine Sonographie, eventuell eine Mammographie und eine True-cut-Biopsie oder FNP. Fallbeschreibung: Anlässlich der Routineuntersuchung bei einer 40-jährigen Patientin wird ein palpabler, druckdolenter Mammaknoten festgestellt. Die persönliche Anamnese sowie die Familienanamnese sind bezüglich Brusterkrankungen bland. In der klinischen Untersuchung findet sich ein ca. $2 \times 2 \mathrm{~cm}$ grosser, druckdolenter, derber, gut verschieblicher Knoten bei $11 \mathrm{~h}$ paraareolär rechts. Die Lymphknotenstationen sind frei. In der Ultraschalluntersuchung präsentiert sich dieser Befund rund, mit scharf begrenzter, glatter Oberfläche, das Echomuster ist hypoechogen und inhomogen, das Austrittsecho verstärkt. In der Feinnadelpunktion finden sich reichlich Blut und Stromazellen, ductale Zellen lassen sich kaum nachweisen. Diese Befunde sind gut vereinbar mit einem Fibroseherd. Da die Patientin sich durch den druckdolenten Knoten gestört fühlt und die Diagnose unsicher ist, entscheidet man sich zur Tumorektomie. In der definitiven Histologie kann die Diagnose «Schwannom» gestellt werden. Schlussfolgerung: Ein Schwannom kann an jedem innervierten Körperteil vorkommen, wenn auch gewisse Regionen bevorzugt betroffen sind. Bei Assoziation mit sensiblen Nervenfasern kann der Knoten druckdolent sein. Die Wahrscheinlichkeit
Abstracts, gynécologie suisse, Lugano, 2005 
der Entartung ist gering. In diesem Fallbeispiel hat sich der unklare Knoten als Schwannom herausgestellt. In der Brust kommen Schwannome extrem selten vor.

\section{P V/52 Senologie \\ Expression de BCL6 dans les Iymphomes primaires du sein (LPS)}

VIastos A.T. ${ }^{\mathrm{a}}$, Pelte M.F. ${ }^{\mathrm{b}}$, Vlastos G. ${ }^{\mathrm{a}}$, Bouchardy C. ${ }^{\mathrm{c}}$, Borisch $B .^{\mathrm{b}}$

aDépartement de Gynécologie et d'Obstétrique, 'bépartement de Pathologie, ' Registre Genevois des Tumeurs, Hôpitaux Universitaires de Genève

Introduction: Les LPS représentent mois de $0,5 \%$ de toutes les tumeurs mammaires. Leur diagnostic, la détermination des facteurs pronostics ainsi que le traitement optimal reste difficile raison pour laquelle cette étude à été conduite. Méthode: Etude rétrospective à partir des datas du registre des tumeurs de notre canton et revues des dossiers entre 1973 et 1998. Caractéristiques générales, états général, comorbidités, traitement et cause de décès ont été analysée. Une analyse histologique par immunohistochimie et les courbes Kaplan-Meier ont complété notre étude. Résultats: 9 cas de LPS dont 2 non primaires ont été répertorié. L'analyse porte sur les 7 restants. L'âge moyen est de 65 ans (39-86). La taille moyen est de $4,5 \mathrm{~cm} .5$ patientes ont un lymphome diffus à large cellule $\mathrm{B}$, 1 patiente a un lymphome folliculaire grade III et 1 patiente présente un lymphome de type MALT. Cliniquement et radiologiquement tous les cas ont été considérés comme cancer du sein au moment du diagnostic. L'aspiration à l'aiguille fine manqua le diagnostic dans 1 cas et l'histologie extemporanée dans 3 cas. Toutes les patientes subirent une mastectomie ou tumorectomie avec dissection axillaire ( 5 cas). 4 patientes bénéficièrent d'un traitement de chimiothérapie adjuvante et une d'une radiothérapie. Le suivi moyen est de 5,8 ans $(0,15-16) .1$ cas développa une récurrence locale et 2 systémique. La survie à 5 et 10 ans est respectivement de 57 et 15\%. Parmi les 3 patientes décédées de leur LPS, le marquage par bcl-2 est positif dans 2 cas mais négatif pour bcl- 6 dans tous les cas. Parmi les 3 survivantes, tous les bcl-2 et bcl-6 sont positifs. Conclusion: LPS est une pathologie des patientes âgées de mauvais pronostic. Le diagnostic de lymphome devrait être établi avant le traitement, mais reste difficile. En effet toutes nos patientes ont subi une chirurgie ce qui n'entre pas dans le traitement les lymphomes. Malgré le petit nombre de cas, cette étude établi bcl-6 comme important facteur pronostic potentiel. Ce qui devra être confirmé par une étude plus large.

\section{P V/53 Senologie \\ P53 as a Prognostic Factor in Primary Breast and Ovarian Tumors: Analysis of Published Clinical Data}

Irminger-Finger I. ${ }^{\mathrm{b}}$, Vlastos A.-T. ${ }^{\mathrm{a}}$, Vlastos G. ${ }^{\mathrm{a}}$, Feki A. ${ }^{\mathrm{a}}$

${ }^{a}$ Department of Gynecology and Obstetrics, ${ }^{b}$ Department of Geriatrics, Biology of Aging Laboratory, Geneva University Hospital

Breast and ovarian cancers, like other cancers, occur due to genetic damage. Research aimed to determine the specific genes involved in the development of breast and ovarian cancers will help to understand how normal breast and ovarian epithelial cells escape regulation of proliferation, apoptosis and senescence. It was deter- mined that approximately $10 \%$ of ovarian cancers and $20-30 \%$ of breast cancers arise in women who have inherited mutations in cancer susceptibility genes such as BRCA1, BRCA2 and other DNA repair genes. The ability to perform genetic testing permits the identification of women at increased risk who can then be offered preventive strategies. The vast majority of ovarian and breast cancers are sporadic, presumably resulting from the accumulation of genetic damage over lifetime. Several genes involved in breast and ovarian carcinogenesis have been identified, most notably the p53 tumor suppressor. The recent availability of expression microarrays has facilitated the simultaneous screening of thousands of genes and this will extend further the understanding of molecular events involved in the dynamic development of ovarian and breast cancers. Then, all this knowledge could be translated into effective screening, surveillance, prevention, and treatment strategies in the future.

\section{P V/54 Senologie \\ Lymphabfluss in Abhängigkeit von der Tumorlokalisation beim Mammakarzinom}

Kleinert D., Müller C., von Orelli S., Fehr M., Fink D.

Klinik für Gynäkologie, Departement Frauenheilkunde, Universitätsspital Zürich

Einführung: Ziel dieser Arbeit war es zu untersuchen, wie der Lymphabfluss beim frühen Mammakarzinom in Abhängigkeit von der Primärtumorlokalisation erfolgt. Material und Methoden: Im Zeitraum von 05/1999 bis 01/2005 wurde an unserer Klinik bei 268 Patientinnen mit Mammakarzinom eine Sentinel-Lymphonodektomie durchgeführt. Die Indikation dazu war gegeben, wenn der Tumor $<3 \mathrm{~cm}$ mass und klinisch eine N0-Situation vorlag. Am Vortag der Operation wurde nach peritumoraler Injektion von $3 \mathrm{ml}$ Nanocol peritumoral und $0,5 \mathrm{ml}$ subdermal von insgesamt $70 \mathrm{MBq}$ eine Lymphszintigrafie durchgeführt. Resultate: Bei 130 (49\%) Patientinnen fand sich der Tumor im oberen äusseren Quadranten, bei $61(23 \%)$ oben innen, bei $34(13 \%)$ unten aussen, bei $22(8,3 \%)$ unten innen und bei $19(7,1 \%)$ zentral. Bei 11 Patientinnen $(4 \%)$ konnte kein Sentinellymphknoten (SLK) szintigraphisch dargestellt werden, bei 8 (3\%) wurde keine Szintigraphie gemacht. Nur axilläre SLK wurden bei 76\% der Patientinnen dargestellt, bei $2 \%$ fanden sich szintigraphisch nur parasternale Lymphknoten. Der Lymphabfluss der Tumoren im oberen äusseren Quadranten erfolgte in $85 \%$ nach axillär, in 5,4\% nach axillär und parasternal, zu $2 \%$ axillär und infraclaviculär und in $0,8 \%$ nur nach parasternal. Bei den Tumoren des unteren äusseren Quadranten fand sich der SLK in 73,5\% axillär, $14,7 \%$ axillär und parasternal. Wenn der Tumor oben innen lag, fand sich der SLK in 65,5\% axillär, in $18 \%$ axillär und parasternal und in 3,3\% parasternal. Bei einer Tumormanifestation im unteren inneren Quadranten fanden sich 63,6\% der SLK axillär, 18,2\% axillär und parasternal und 9\% parasternal. Die zentralen Tumore drainierten in 73,7\% nach axillär, in 5,3\% nach axillär und parasternal und in 5,3\% nach parasternal. Schlussfolgerung: Bei den meisten Patientinnen (94\%) lassen sich bereits szintigraphisch axilläre Sentinellymphknoten darstellen. Ausschliesslich parasternale Lymphknoten zeigten sich nur in 2\%. Erwartungsgemäss findet sich ein parasternaler Lymphabfluss am häufigsten bei Primärtumoren im inneren unteren Quadranten. Die zentralen Mammakarzinome dagegen drainieren eher wie die Karzinome des oberen äusseren Quadranten. 


\section{P V/55 Senologie \\ Histologische Unterschätzung der atypisch duktalen Hyperplasie in der stereotaktischen Brustbiopsie}

Pauli E., von Orelli S., Fehr M., Fink D.

Gynäkologische Klinik, Departement Frauenheilkunde, Universitätsspital Zürich

Einführung: Die stereotaktische Brustbiopsie klinisch okkulter, suspekter Mammographiebefunde kommt immer häufiger zur Anwendung. Dadurch nimmt die Diagnose der atypisch duktalen Hyperplasie (ADH) zu. Die ADH ist mit einem 4- bis 5-fach erhöhten Risiko der malignen Entartung assoziiert und gilt als Präkanzerose. Ein Problem stellt die histologische Unterschätzung bei der ADH dar. Material und Methode: Am Brustzentrum des Universitätsspitals Zürich werden sämtliche Brustbiopsien prospektiv erfasst. Vom 11.08.2000 bis 9.02.2005 wurden 527 stereotaktische Brustbiopsien entnommen, wovon 335 vakuumassistierte Stanzbiopsien [Mammotome $^{\circledR}$ ] und 192 Schneiderohrbiopsien [SiteSelect ${ }^{\circledR}$ ] waren. 134 Biopsien erfolgten durch ultraschallgesteuerte Stanzbiopsie [Mammotome ${ }^{\circledR}$ hand held]. Beim Vorliegen einer ADH wurde die offene Biopsie empfohlen. Ergebnisse: Von 527 stereotaktischen und 134 ultraschallgesteuerten Mammotome ${ }^{\circledR}$-Biopsien wurde bei $27(5,1 \%)$ resp. 4 Patientinnen $(3,0 \%)$ eine ADH diagnostiziert. 24 mammographische Befunde $(88,9 \%)$ entsprachen BIRADS-4 Läsionen, 3 BIRADS-3 Läsionen (11,1\%). 40,7\% der ADH waren radiologisch vollständig, 29,6\% partiell $>50 \%$ und $14,8 \%$ partiell $<50 \%$ entfernt worden. Die Inzidenz der histologischen Unterschätzung der ADH in der Stereotaxie betrug 25,9\% $(n=7)$, wovon in 4 Fällen ein DCIS und in 3 Fällen ein invasives Karzinom vorlag. $57,1 \%$ der in der Stereotaxie unterschätzten $\mathrm{ADH}$ waren partiell $>50 \%$ und $14,3 \%$ vollständig biopsiert worden. Bei den Mammotome ${ }^{\circledR}$-hand-held-Biopsien betrug die histologische Unterschätzung $50 \%(n=2)$, wobei es sich zweimal um ein DCIS handelte. Schlussfolgerungen: Angesichts der hohen Inzidenz der histologischen Unterschätzung in der stereotaktischen Biopsie ist bei der atypisch duktalen Hyperplasie die offene Nachresektion zu empfehlen. Nur im Ausnahmefall, d.h. bei vollständig entferntem Befund und nur einzelnen Herden einer ADH, ist ein expektatives Vorgehen mit halbjährlichen Kontrollmammographien vertretbar. Die Patientin sollte aber über das erhöhte Risiko der malignen Entartung der ADH aufgeklärt werden.

\section{P V/56 Senologie \\ Indication du prélèvement du ganglion sentinelle chez une patiente agée atteinte d'un cancer du sein et d'un lymphome non-Hodgkinien}

Rombaldi F., Schäffer P., Pelte M.F., Dubuisson J.B., Vlastos G.

Service de Sénologie du Département de Gynécologie et d'Obstétrique et Service de Pathologie, Hôpitaux Universitaires de Genève

Introduction: Le cancer du sein est très fréquent chez la femme âgée, mais rarement associé avec d'autres cancers. A noter que le prélèvement du ganglion sentinelle est le traitement de choix pour évaluer l'invasion du ganglion sentinelle. Cas clinique: Nous rapportons le cas d'une patiente âgée de 93 ans, avec une anamnèse familiale de cancer du sein, aux antécédents d'hypertension et d'infarctus du myocarde. Durant le contrôle annuel son gynécologue a palpé un nodule suspect situé dans le quadrant supéro-externe gau- che. Elle a bénéficié d'une mammographie qui montre une image stellaire, puis d'une biopsie échoguidée de la lésion qui parle pour un carcinome canalaire invasif. Une tumorectomie, le prélèvement du ganglion sentinelle ont été pratiqués. L'aspect du ganglion sentinelle était macroscopiquement normal mais il était entouré de ganglions lymphatiques d'aspect suspect, raison pour laquelle un curage axillaire a été effectué. L'histopathologie a conclu à un ganglion sentinelle sans tumeur, mais à l'invasion des autres ganglions par un lymphome non-Hodgkinien de type «en manteau» de stade avancé, qui fait partie d'une forme rare qui se développe à partir de la zone du manteau des nodules lymphatiques. Les immunophénotypes sont CD5+, CD20+, CD43+, CD23-. Une translocation 11-14 est recherchée. Discussion: Le lymphome non-Hodgkinien et le cancer du sein sont rarement associés sauf après une chimiothérapie ou une radiothérapie. Un seul cas a été décrit dans la littérature a été publié dans le World J Surg Oncol 2003. Notre cas met en évidence le drainage par deux voies lymphatiques différentes du cancer du sein et du lymphome non-Hodgkinien. Le curage axillaire était probablement superflu. La chimiothérapie a été refusée par la patiente. Conclusion: En conclusion, le prélèvement du ganglion est possible dans les cas de cancer du sein et les lymphomes non-Hodgkinien.

\section{P V/57 Senologie \\ Auftreten einer isolierten Skelettmetastase im OS Metacarpale III links bei Mamma- karzinom - Ein Fallbericht}

Eitel K., Eberhard M., Litschgi M.

Frauenklinik Kantonsspital Schaffhausen

Einleitung: Skelettmetastasen treten meist bei Patienten mit fortgeschrittenen Karzinomen auf, insbesondere bei Lungen-, Brustund Prostatakrebs. Weniger als 0,1\% der Skelettmetastasen treten in Knochen der Hände und Füsse auf. Das Fehlen von rotem Knochenmark in diesen Knochen wird für die niedrige Inzidenz verantwortlich gemacht. Fall: Eine 58jährige Patientin mit einem 8 Jahre zuvor diagnostizierten invasiv duktalen Mammakarzinom beidseits stellte sich mit einer seit zwei Monaten bestehenden, schmerzhaften Schwellung des Metacarpo-Phalangealgelenkes III links nach einem Bagatelltrauma vor. Im Vorjahr wurden supraclaviculäre und mediastinale Lymphknotenmetastasen, pulmonale Metastasen und eine Nebennierenmetastase diagnostiziert. Kernspintomographie, Computertomographie und konventionelles Röntgen ergaben einen hochgradigen Verdacht auf eine im Mark-raum des Metacarpale III links gelegene Metastase des bekannten Mammakarzinoms. Skelettszintigraphisch zeigte sich eine intensive Mehrbelegung des Metacarpale III links ohne Hinweis für weitere Skelettmetastasen. Die Diagnose isolierte Skelettmetastase im Metacarpale III links wurde bioptisch gesichert. Therapeutisch wurde im betroffenen Bereich mit $11 \cdot 3$ Gy $=33$ Gy über drei Wochen radiotherapiert. Dadurch wurde subjektiv eine funktionelle Verbesserung der linken Hand und Schmerzfreiheit erzielt. Im Verlauf traten ein halbes Jahr nach Diagnosestellung der isolierten Skelettmetastasen disseminierte cerebrale Metastasen auf, jedoch keine weiteren Skelettmetastasen. Die Patientin verstarb ein knappes Jahr nach Diagnosestellung der isolierten Skelettmetastase. Zusammenfassung: Weniger als 0,1\% aller Skelettmetastasen treten in den Akren auf, am häufigsten bei Patienten mit Lungenkarzinom (47\%) und Brustkrebs (12,5\%). Bei einer Medline-Recherche fanden wir im Zeitraum 1960-2005 10 Fallberichte mit Akrometastasen bei Brustkrebs. Differentialdiagnostisch muss bei Brustkrebs-patientinnen mit Schmerzen in Hand/ Fuss an Metastasen gedacht werden. Die Prognose der betroffenen Patienten ist schlecht, die Überlebensdauer nach Diagnosestellung
Abstracts, gynécologie suisse, Lugano, 2005 
einer isolierten Handknochenmetastase beträgt im Mittel 5,3 Monate. Die Behandlung ist rein palliativ. Therapeutisch werden Radiotherapie, Chemotherapie und chirurgische Therapien eingesetzt.

\section{P V/58 Senologie \\ Valeur de la biopsie du ganglion sentinelle chez les patientes présentant un carcinome canalaire in situ du sein (CCIS)}

Delaloye J.F. ${ }^{\mathrm{a}}$,Warkus T. ${ }^{\mathrm{a}}$, Brunisholz Y. ${ }^{\mathrm{a}}$, Monod J.F. ${ }^{\mathrm{b}}$, Fiche M. ${ }^{c}$, De Grandi P. ${ }^{\mathrm{a}}$, Bischof Delaloye A. ${ }^{\mathrm{d}}$

aDépartement de Gynécologie-Obstétrique, Centre Hospitalier Universitaire Vaudois, Lausanne, 'bervice de Gynécologie, Ensemble Hospitalier de la Côte, Morges, 'Institut

Universitaire de Pathologie, dService de Médecine nucléaire, Centre Hospitalier Universitaire Vaudois, Lausanne

Introduction: Le carcinome canalaire in situ (CCIS) représente $15-20 \%$ des carcinomes du sein nouvellement diagnostiqués. Des métastases ganglionnaires axillaires ont été rapportées dans 1,8 à $13 \%$ des cas, résultant le plus souvent de la présence d'une microinvasion méconnue. Le but de cette étude a été de déterminer l'incidence des (micro)-métastases dans les ganglions axillaires des patientes présentant un DCIS. Matériel et méthodes: Soixante patientes porteuses d'un CCIS diagnostiqué par une micro-biopsie transcutanée ont subi soit une tumorectomie, soit une mastectomie combinée à une biopsie du ganglion sentinelle. Les ganglions sentinelles ont été inclus in toto et étudiés sur des coupes réalisées tous les $250 \mathrm{~mm}$, colorées par hématoxyline-éosine (HE) et en immunohistochimie (anticorps $\mathrm{C} 11$ anti-cytokératines). La découverte d'une (micro)-métastase a conduit à pratiquer un complément de dissection axillaire du niveau I de Berg. Résultats: Aucune (micro)métastase a été diagnostiquée chez 55/60 patientes (92\%). Le CCIS était pur (sans carcinome invasif associé) chez 53/60 patientes. Quatre micro-métastases et une métastase ont été identifiées chez $5 / 60$ patientes $(8 \%)$. Chez ces 5 patientes, l'examen de la pièce opératoire a mis en évidence une composante de carcinome invasif associée, la taille du CCIS variait de 3,5 à $10,5 \mathrm{~cm}$, le CCIS était 4 fois palpable et le grade nucléaire était bien (G1) à peu (G3) différencié. Aucune (micro-)métastase n'a été trouvée dans le complément de curage axillaire. Conclusions: Chaque fois que des cellules tumorales ont été retrouvées dans les ganglions sentinelles de CCIS, l'examen complet de la pièce opératoire a révélé un carcinome invasif. La conversion du CCIS en carcinome invasif a été observée uniquement dans les CCIS mesurant $>3,5 \mathrm{~cm}$. La biopsie du ganglion sentinelle est appropriée si le CCIS est palpable ou s'il impose une mastectomie.

\section{P V/59 Senologie \\ Utilisation plus importante de traitements systémiques chez les femmes atteintes d'un cancer du sein avec une histoire familiale sévère: Résultats d'une étude de population \\ Verkooijen H.M. ${ }^{\mathrm{a}}$, Chappuis P.O. ${ }^{\mathrm{b}}$, Rapiti E. ${ }^{\mathrm{a}}$, Vlastos G. ${ }^{\mathrm{c}}$, Fioretta G. ${ }^{\mathrm{a}}$, Sarp S. ${ }^{\mathrm{a}}$, Sappino A.P. ${ }^{\mathrm{b}}$, Schubert H. ${ }^{\mathrm{a}}$, Bouchardy C. \\ ${ }^{a}$ Registre Genevois des Tumeurs, ${ }^{b}$ Oncologie Médicale, ${ }^{\mathrm{C}}$ Gynécologie et Obstétrique, Hôpitaux Universitaires de Genève}

Introduction: Un facteur de risque important pour le cancer du sein est la survenue de cancers du sein ou de l'ovaire dans une même famille. Dans cette étude de population, nous avons évalué l'impact de l'histoire familiale sur les méthodes de détection, le diagnostic précoce et les choix thérapeutiques des patientes atteintes d'un cancer du sein de manière précoce. Matériel et méthodes: En utilisant le registre familial, nous avons identifié toutes les patientes âgées de $<50$ ans, diagnostiquées entre 1990 et 2001. Nous avons comparé les patientes avec un risqué familial élevé (défini par des cancers du sein/ovaire chez: $>1$ parent de premier degré $<50$ ans, $>2$ parents de premier degré, ou $>3$ parents de premier ou second degré) à des patientes sans parents affectés. Nous avons comparé les caractéristiques tumorales, les méthodes de détection et de traitements par une analyse de régression logistique multivariée. Résultats: Ont été incluses dans cette étude, 633 patientes avec un cancer du sein précoce et 58 patientes avec un risque familial élevé. Ces dernières n'étaient pas diagnostiquées ni plus fréquement, que ce soit par surveillance ou dépistage, ni à un stade plus précoce par rapport aux patientes à faible risque. Les patientes à haut risque étaient traitées chirurgicalement de manière conservative, mais aussi par des mastectomies bilatérales. L'hormonothérapie était plus utilisée (adjusted odds ratio 1,9,95\% CI: 1,1-3,4). De plus, la chimiothérapie et l'hormonothérapie étaient plus utilisées pour des patientes avec des ganglions négatifs ou des tumeurs avec des récepteurs hormonaux négatifs $(\mathrm{p}<0,05)$. Conclusions: Dans cette étude de population, une histoire familiale importante de cancer du sein est associée à une utilisation plus accrue de traitements systémiques chez des patientes atteintes d'un cancer du sein précoce.

\section{P VI/60 Senologie/Endokrinologie Bilanz eines privaten IVF-/ICSI-Anbieters 1999-2004}

Pavic N.

Fertilitas: Privates IVF-/ICSI-Zentrum, Josefsklinik Basel

Einführung: Die Leistungsfähigkeit und sogar Existenzberechtigung kleinerer IVF-Zentren wird gelegentlich in Frage gestellt. Als bisher mit Transportsystem arbeitender 'part-time' 5 Reproduktionsmediziner habe ich die Behandlung von 100 konsekutiven Paaren zusammengestellt. Beratung, Zusatzdiagnostik, Vorbehandlung und teilweise Follikelpunktion erfolgten in üblicher Weise in der Praxis. Die Aspirate wurden innert $<30 \mathrm{~min}$ ins IVF-/ICSILabor der Viollier AG transportiert. Dort erfolgte die extrakorporale Befruchtung und der Embryotransfer (ET), letzterer durch den Autor. Auftautransfers wurden im sog. künstlichen Zyklus vorgenommen. Patientengut und Methode: Retrospektive Analyse der Daten aller vom Jan. 99 bis Okt. 04 von mir persönlich behandelten Paare $(n=100)$. Diese Daten sind auch in die FIVNAT-CHStatistik eingegangen und überprüfbar. Folgende Informationen wurden erfasst: Alter der Frau und Sterilitätsdauer (zum Zeitpunkt der ersten Follikelpunktion (FP) bei uns), primäre/sekundäre Sterilität, Indikation(en) für ICSI/IVF. Anzahl FP pro Patientin. Ort der FP (Klinik/Praxis). Anzahl ET. Anzahl Embryonen pro ET aufgeteilt in Frisch- bzw. Auftau-ET's. Zum Outcome: Komplikationen: der FP, Hyperstimulationssyndrom (HSS). Klinische Aborte (nach US mit Fruchtblase), Geburten und Schwangerschaften $>20$. SSW. Mehrlinge. Schwangerschafts- und Geburtskomplikationen. Morbidität/Missbildungen der Kinder. Drop-outs. Therapieunabhängige Geburten. Ergebnisse: Insgesamt haben bis jetzt 41 der 100 Patientinnen mindestens ein lebendes Kind geboren. 6 Patientinnen erreichten bisher nur klinische Aborte. 2 Frauen erlitten nur einen Partus immaturus $>22$. SSW. 8 Patientinnen haben nach ICSI zweimal geboren. Bei 11 von 41 Geburten (27\%) handelte es sich um Gemelli. Hospitalisationsbedürftige Frühkomplikationen 
und HSS traten keine auf. Von 61 Kindern wiesen 2 (operable) Fehlbildungen auf. Es wird versucht herauszuarbeiten, wodurch sich die schliesslich erfolgreichen Paare von den nicht erfolgreichen unterscheiden. Schlussfolgerung: Unter Berücksichtigung der Tatsache, dass ein beträchtlicher Teil der Paare noch im Programm ist, ist bei uns eine kumulative Lebendgeburtenrate von ca. 50\% pro Patientin realistisch. Damit sind unsere Resultate durchaus vorzeigbar. Unsere Kontinuationsrate (der Anteil Paare, der sich mehrmals behandeln lässt) liegt über dem CH-Durchschnitt.

\section{P VI/61 Senologie/Endokrinologie \\ Brustdurchblutung bei Frauen mit oder ohne hormonaler Kontrazeption}

Hornung R. ${ }^{a}$, Matzinger B. ${ }^{\mathrm{a}}$, Brown D. ${ }^{\mathrm{b}}$, Stahel M.C. ${ }^{\mathrm{a}}$, Wolf M. ${ }^{\mathrm{b}}$, Bucher H.U. ${ }^{\mathrm{b}}$, Fink D. ${ }^{\mathrm{a}}$

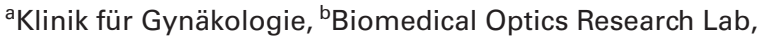
Klinik für Neonatologie, Universitätsspital Zürich

Einführung: Frequency-domain near-infrared spectroscopy (FD-NIRS)-Messungen ermöglichen die nicht-invasive Bestimmung von optischen Eigenschaften, d.h. Absorption und Streuung von Licht, im Brustgewebe. Ziel dieser Studie war, die Brustdurchblutung in Abhängigkeit des Menstruationszyklus bei Frauen mit oder ohne oraler Kontrazeption zu untersuchen. Material und Methoden: Gesunde Probandinnen mit oder ohne oraler Kontrazeption wurden untersucht. Die optischen Eigenschaften der Brüste wurden an 12 Zyklustagen mittels eines FD-NIRS-Instrumentes bestimmt. Daraus wurden die Konzentrationen an Oxy- und Deoxyhämoglobin, totalem Hämoglobin, Sauerstoffsättigung $\left(\mathrm{StO}_{2}\right)$, und Wasser berechnet. Hier wird stellvertretend nur die $\mathrm{StO}_{2}$ gezeigt. Resultate: Während die $\mathrm{StO}_{2}$ bei Probandinnen unter oraler Kontrazeption konstant bleibt, scheint die $\mathrm{StO}_{2}$ bei Probandinnen in der spontanen Lutealphase abzusinken. Schlussfolgerung: FDNIRS ist eine Methode, welche fähig ist, hormoninduzierte Veränderungen der Sauerstoffsättigung in der Brust zu messen. Diese Daten werden uns das Monitoring der Behandlung von Mastodynie, einem Schmerz- und Spannungsgefühl der Brüste, ermöglichen.

\section{P VI/62 Senologie/Endokrinologie Messung der Brust-Perfusion während dem Menstruationszyklus mittels Frequency-Domain Near-Infrared Spectroscopy (FD-NIRS)}

Stahel M.C. ${ }^{\mathrm{a}}$, Brown D. ${ }^{\mathrm{b}}$, Matzinger B. ${ }^{\mathrm{a}}$, Wolf M. ${ }^{\mathrm{b}}$, Bucher H.U. ${ }^{\mathrm{b}}$, Fink D. ${ }^{\mathrm{a}}$, Hornung R. ${ }^{\mathrm{a}}$

${ }^{a}$ Klinik für Gynäkologie, ${ }^{b}$ Biomedical Optics Research Lab, Klinik für Neonatologie, Universitätsspital Zürich

Einführung: Frequency-domain near-infrared spectroscopy (FD-NIRS)-Messungen ermöglichen die nicht invasive Bestimmung von optischen Eigenschaften, d.h. Absorption und Streuung von Licht, im Brustgewebe. Ziel dieser Studie war, die Brustdurchblutung und das Ödem der Brüste in Abhängigkeit des Menstruationszyklus bei Frauen ohne orale Kontrazeption zu untersuchen. Material und Methoden: Gesunde und nicht-schwangere Probandinnen wurden untersucht. Die optischen Eigenschaften der Brüste wurden an 12 Zyklustagen mittels eines FD-NIRS-Instrumentes bestimmt. Daraus wurden die Konzentrationen an Oxy-und Deoxyhämoglobin, totalem Hämoglobin (totHb), Sauerstoffsättigung, und Wasser $\left(\mathrm{H}_{2} \mathrm{O}\right)$ berechnet. Hier werden stellvertretend nur die tot $\mathrm{Hb}$ und die $\mathrm{H}_{2} \mathrm{O}$ gezeigt. Resultate: Gezeigt werden der relative $\mathrm{H}_{2} \mathrm{O}$ - und totHb-Gehalt der Brust von gesunden Probandinnen als Funktion des Menstruationszyklus. $\mathrm{H}_{2} \mathrm{O}$ scheint in der Lutealphase am höchsten zu sein. Das totHb scheint in der Lutealphase etwas abzusinken. Schlussfolgerung: FD-NIRS-Messungen sind ein vielversprechendes diagnostisches Instrument, um nicht invasiv Durchblutungsveränderungen des Brustgewebe zu detektieren. totHb (d.h. Perfusion) und Wassergehalt der Brüste können mit dem Menstruationszyklus korreliert werden. Diese präliminären Grundlagendaten werden uns die Untersuchung der Mastodynie, einem Schmerz- und Spannungsgefühlsyndrom der Brüste, ermöglichen.

\section{P VI/63 Senologie/Endokrinologie Implanon und Schwangerschaft}

Müller A., Stucki D., Spörri S.

Klinik für Gynäkologie und Geburtshilfe,

Kantonsspital Freiburg

Einführung: Implanon ${ }^{\circledR}$ ist ein subdermales Implantat zur hormonalen Kontrazeption und besteht aus einem Kunststoffstäbchen mit dem Wirkstoff Etonogestrel, welcher kontinuierlich in die Blutbahn abgegeben wird. In der Literatur werden wenige Schwangerschaften unter Implanon ${ }^{\circledR}$ beschrieben. Material und Methoden: Literaturrecherche und Fallbeispiel aus unserer Klinik: Eine 36jährige Patientin wird uns von ihrem Gynäkologen zum Schwangerschaftsabbruch zugewiesen. Die Patientin benutzt seit mehr als 2 Jahren Implanon ${ }^{\circledR}$. Sie berichtet über eine korrekte und termingerechte Implantation durch ihren Gynäkologen, bestätigt regelmässigen Geschlechtsverkehr und verneint eine zusätzliche Medikamenteneinnahme. Implanon ${ }^{\circledR}$ wurde am Tage der festgestellten Schwangerschaft entfernt und der Firma Organon ${ }^{\odot}$ zur Analyse zugesandt. Ergebnisse: Einige der in der Literatur beschriebenen Schwangerschaften wurden durch Organon ${ }^{\odot}$ untersucht, es konnte aber kein Versagen von Implanon ${ }^{\circledR}$ festgestellt werden. Diese Schwangerschaften mussten gemäss den Untersuchungen bereits vor Implantation bestanden haben. In Australien kam es nach Einführung von Implanon ${ }^{\circledR}$ innerhalb von 18 Monaten zu ungefähr 100 ungewollten Schwangerschaften. Diese Fälle wurden analysiert und ein sogenanntes «risk management» etabliert. Als mögliche Gründe für die Schwangerschaften gelten: (1) Fälschlicherweise keine Implantation von Implanon ${ }^{\circledR},(2)$ falscher Zeitpunkt der Implantation von Implanon ${ }^{\circledR}$, (3) Medikamenten-Interaktion, (4) Implanon ${ }^{\circledR}$-Versager. Durch die Anwendung einer Checkliste können die obengenannten Ursachen einer ungewollten Schwangerschaft weitgehend minimiert werden. Schlussfolgerung: An unserer Klinik betreuen wir Patientinnen mit Wunsch nach einer langfristigen Kontrazeption mittels Implanon ${ }^{\circledR}$. Mithilfe einer Checkliste minimieren wir die Risiken einer ungewollten Schwangerschaft. Die Checkliste beinhaltet eine genaue Anamnese mit Schwerpunkt auf Medikamenteneinnahme und Allergien, den 2-3-monatigen Versuch mit Cerazette ${ }^{\circledR}$ zum Ausschluss unerwünschter Nebenwirkungen sowie dem richtigen Festlegen des Implantationszeitpunktes. Dank genauer Dokumentation der Risikofaktoren, dem Implantationszeitpunkt und einer vorschriftsmässigen Implantation von Implanon ${ }^{\circledR}$ können die Fehlerquellen minimiert werden.
Abstracts, gynécologie suisse, Lugano, 2005 


\section{P VI/64 Senologie/Endokrinologie Juveniles, multifokales Fibroadenom versus Phylloidestumor der adoleszenten Mamma}

\author{
Gross N. ${ }^{\mathrm{a}}$, Bannwart $F^{\mathrm{b}}$, Pok J. ${ }^{\mathrm{c}}$, Bürki N. ${ }^{\mathrm{a}}$
}

${ }^{a}$ Frauenklinik, Spital Limmattal, bPathologie, Stadtspital Triemli, 'Departement Frauenheilkunde, Universitätsspital Zürich

Einleitung: Ein Phylloidestumor ist ein seltener Tumor, der typischerweise im Alter von 40 Jahren auftritt. Während der Zeit von 1/95-12/03 wurde in der gynäkologischen Frauenklinik USZ nur 1 Patientin unter 18 Jahren wegen einem Phylloidestumor ambulant betreut. Juvenile Fibroadenome sind dagegen typische Tumoren in der Adoleszenz, welche in der Regel aber solitär auftreten. Kasuistik: Die 15 5/12 jährige Adoleszentin wurde wegen Vd.a. ein Rezidiv eines Phylloidestumors zugewiesen. Vor 14 Monaten im 10/03 wurde bei der Patientin durch einen plastischen Chirurgen bei Vd.a. ein Fibroadenom ambulant in LA eine Mamma-PE links durchgeführt. Die Histologie wurde als $4 \mathrm{~cm}$ grosser Phylloidestumor, low grade klassiert. Bereits 2 Monate postoperativ zeigte sich in der linken Mamma bei reizloser, periareolärer Narbe ein in der 2-Uhr-Achse liegender, derber, unebener, indolenter, mobiler Knoten von ca. $2 \mathrm{~cm}$ DM. Das zytomorphologische Bild der mehrmaligen FNP ergab keinen AP für ein Hämatom oder Rezidiv eines Phylloidestumors. Im Einverständnis mit der Adoleszentin wurde ein abwartendes Verhalten gewählt. Bei klinisch und sonografisch verifizierter Grössenprogredienz des Tumors über die letzten 3 Monate entschied man sich bei Vd.a. ein Rezidiv des Phylloidestumors zur Mamma-PE links bei 2 Uhr. Histologisch wurde der $3 \mathrm{~cm}$ grosse Tumor nun als vollständig exzidiertes juveniles Fibroadenom beurteilt und die Diagnose des ersten Tumors revidiert. Im umgebenden Mammagewebe fanden sich vereinzelt fibroadenomatöse Herde, was zu einem multifokalen Auftreten des juvenilen Fibroadenoms passt. Schlussfolgerung: Juvenile Fibroadenome der Mamma treten in der Regel als solitäre Tumoren auf, multiples Auftreten ist selten. Die Abgrenzung zum Phylloidestumor ist wichtig, um Übertherapien zu vermeiden. Juvenile Fibroadenome sollten in der Adoleszenz sehr sparsam exzidiert werden, um die Brustentwicklung nicht zu gefährden, während der Phylloidestumor gut im Gesunden reseziert sein muss, um Rezidive zu vermeiden. Der Abgrenzung der beiden Entitäten kommt damit grosse Bedeutung zu und im Zweifelsfalle sollte die Diagnose eines rezidivierenden Phylloidestumors im Adoleszentenalter vor weiteren chirurgischen Eingriffen überprüft werden.

\section{P VI/65 Senologie/Endokrinologie Fausses élévations de progestérone (P4) en fin de stimulation ovarienne pour FIV}

Romoscanu I. ${ }^{\mathrm{a}}$, Stanczyk $F^{\mathrm{b}}$, Bourgain C. ${ }^{\mathrm{c}}$, Weintraub J. ${ }^{\mathrm{a}}$, Paulson . $^{\mathrm{b}}$, de Ziegler $D^{\mathrm{a}}$

aMédecine de la Reproduction, Hôpitaux Universitaires de Genève, 'bSC, Los Angeles, Calif., USA, 'bUB, Bruxelles, Belgique

Introduction: Les élévations de $\mathrm{P} 4$ en fin de SO pour FIV existent malgré les agonistes du GnRH (GnRH-a). Le don d'ovocytes a démontré que ceci n'a pas d'effets sur la qualité ovocytaire. Une altération endométriale avec accélération des transformations sécrétoires et fermeture prématurée de la fenêtre de réceptivité a par contre été évoquée, avec taux de grossesse bas en cas de réponse ovarienne faible, mais sans effet en cas de réponse forte [FS 1997;
68:799]. Pour comprendre pourquoi les élévations de P4 en fin de SO sont sans effet en cas de réponse forte, nous avons étudié 2 patientes présentant une élévation prolongée de $\mathrm{P} 4$ dont la ponction a été annulée (risque d'hyperstimulation). Matériel et méthode: 2 patientes FIV recevant un protocole FSH-GnRH-a ont eu des mesures directes (MD) de P4 comprises entre 2.3 et $5 \mathrm{ng} / \mathrm{ml}$ en fin de SO. Les $\mathrm{SO}$ ayant été stoppées pour risque d'hyperstimulation ( $>10$ foll./ovaire), une biopsie endométriale a été effectuée et lue par deux pathologistes (JW et CB) indépendants. La P4 a été remesurée secondairement par une méthode analytique (MA) avec extraction et séparation sur colonne de célite. Résultats: En dépit d'une élévation de $\mathrm{P} 4$ de 2,3 à $5 \mathrm{ng} / \mathrm{ml}$ pendant $4 \mathrm{~J}$ au moins, aucune transformation sécrétoire de l'endomètre n'a été identifiée chez les patientes. Pour les 4 derniers jours de la $\mathrm{SO}$, les résultats de P4 (MD/MA) ont montré respectivement 2,3/0,8, 2,3/1,07, $2,4 / 0,94,3,3 / 1,06$ et $2,6 /-, 4,2 /-, 5 / 2,45,3,5 / 2,04$ pour ces 2 patientes, les valeurs fournies par MA sont en moyenne de $66 \%$ et $47 \%$ inférieures à celles fournies par la MD. Conclusion: L'élévation de la $\mathrm{P} 4$ entre 2,3 et $5 \mathrm{ng} / \mathrm{ml}$ (MD) pendant les 4 derniers jours de SO pour FIV n'a eu aucune conséquence sur l'endomètre de 2 femmes dont la réponse ovarienne était forte. Un dosage analytique a démontré que les taux réels de P4 étaient beaucoup plus bas, ce qui explique l'absence d'effet endométrial. Le dosage direct de la P4 en fin de SO pour FIV est donc inutile en cas de forte réponse ovarienne. La fausse élévation de $\mathrm{P} 4$ en fin de $\mathrm{SO}$ avec réponse forte reflète probablement une augmentation de production de métabolites inactifs de la P4.

\section{P VI/66 Senologie/Endokrinologie \\ Cryothérapie sous IRM du cancer du sein: résultats préliminaires}

Vlastos G. ${ }^{\mathrm{a}}$, Kinkel K. ${ }^{\mathrm{b}}$, Pelte M.F. ${ }^{\mathrm{c}}$,Terrier $F^{\mathrm{b}}$

Départements de a Gynécologie et d'Obstétrique,

${ }^{\mathrm{b}}$ Radiologie, 'Pathologie, Hôpitaux Universitaires de Genève

Introduction: La chirurgie du cancer du sein évolue vers des approches minimalement invasives. La cryothérapie est une modalité acceptée dans le traitement de nombreuses tumeurs. Toutefois, son utilisation dans le traitement du cancer du sein est encore expérimentale. L'objectif de cette étude est d'évaluer l'utilisation de la cryothérapie sous résonance magnétique nucléaire (IRM) dans le traitement du cancer du sein et de prédire la destruction tumorale par ultrasons ou IRM. Matériel et méthode: Des patientes diagnostiquées avec des tumeurs invasives du sein de $<2 \mathrm{~cm}$ (prouvées par biopsies) ont été traitées par cryothérapie sous IRM en anesthésie locale. Une excision chirurgicale des lésions a été réalisée après 4 semaines. Toutes les patientes ont bénéficiée d'une IRM à bas champs et d'ultrasons avant la cryothérapie et avant chirurgie définitive. Les pièces ont été orientées et photographiées. Les dimensions anatomopathologiques ont été comparées aux dimensions obtenues par l'imagerie. Des cytokératines ont été réalisées pour vérifier la viabilité tumorale. Résultats: Dix patientes ont été traitées par cryothérapie. Le traitement a été accepté. La taille tumorale clinique était de $1,5 \mathrm{~cm}(0,8-2 \mathrm{~cm})$. A l'exception d'une ecchymose minime du sein, aucune complication immédiate n'a été rapportée. Toutefois 3 patientes ont présentés des brûlures cutanées. L'évaluation de la destruction tumorale a été totale dans certains cas ( 3 cas) ou partielle ( $7 \mathrm{cas}$ ), avec persistance de cellules tumorales, de type in situ. L'expression des cytokératines 8/18 était négatif dans les cellules tumorales. L'ultrason a permis de prédire le volume tumoral résiduel chez toutes les patientes (sensitivité $100 \%$, spécificité $0 \%$ ) et l'IRM respectivement chez 7 patientes 
(sensitivité 100\%, spécificité 66\%). Conclusion: La cryothérapie sous IRM est un traitement minimalement invasif du cancer du sein très prometteur. Toutefois la destruction tumorale doit être encore améliorée.

\section{P VI/67 Senologie/Endokrinologie Dosage de I'hormone anti-Mullérian sérique au cours du cycle menstruel et sous contraception hormonale combinée}

Ibecheole V., Fondop J.J., Ventura P., de Candolle G., Bischof P., de Ziegler D.

Hôpitaux Universitaires de Genève

Introduction: Des études récentes suggèrent que l'AMH est le meilleur marqueur pour l'issue de la FIV et probablement le plus fiable pour évaluer la réserve ovarienne. L'AMH, un membre de la famille des TGF, est produit par les cellules de la granulosa des follicules antraux. Les données indiquent que l'AMH inhibe la croissance des follicules primordiaux, influence négativement l'aromatase, exerce une action paracrine sur les cellules de la granulosa et probablement sur les ovocytes. La production de l'AMH est indépendante de la FSH, par contre on observe une diminution dans les stimulations ovariennes contrôlées, suite à l'administration de l'hCG. Ceci est probablement à mettre en relation avec une réduction de la croissance folliculaire. Les valeurs de l'AMH sont augmentées dans les situations de syndrome des ovaires polykystiques et d'aménorrhée hypothalamique. Afin d'améliorer nos connaissances, nous avons mesuré l'AMH chez des femmes, soit pendant le cycle menstruel soit sous contraception hormonale combinée. Méthodes: L'oestradiol (E2) et la FSH ont été mesurés par un automate (Kryptor, Brahms, Berlin, Germany) aux jours $-10,-5,-2,1$, 2, 10 par rapport au pic LH. La mesure de l'AMH a été réalisée by ELISA (EIA AMH/MIS Beckman Coulter). Résultats: Les résultats montrent que le taux d'AMH reste relativement stable pendant la phase précoce et tardive du cycle menstruel $(26,4 \mathrm{p} M / 1)$, alors qu'il est abaissé après l'ovulation (22,2 pM/l). Lorsqu'on compare les résultats entre les deux populations, on constate que la moyenne du taux d'AMH est similaire. Cependant on assiste à une plus grande variabilité des valeurs chez les femmes qui sont sous contraception hormonale combine $(0,65-74,5$, min-max). Conclusion: Par cette étude, nous pouvons valider la possibilité d'effectuer des dosages d'AMH pour évaluer la réserve ovarienne sous contraception hormonale. Toutefois en raison d'une grande variabilité des données chez ces femmes, une étude à plus grande échelle serait souhaitable.

\section{P VI/68 Senologie/Endokrinologie \\ ROLL (Radioguided Occult Lesion Localisation) bei nicht palpablen Mammakarzinomen oder DCIS; Erfahrungen der ersten 15 Fälle}

Sarlos D., Schär G.

Frauenklinik Kantonsspital Aarau

Einführung: Ein recht grosser Anteil der Mammakarzinome und die meisten insitu-Karzinome welche operiert werden, sind nicht palpabel. Diese Zahl hat durch das häufiger durchgeführte individuelle Screening, die verbesserten mammographischen Techniken und die stereotaktischen Brustbiopsien in den letzten Jahren deutlich zugenommen. Die konventionellen präoperativen Drahtmarkierungen waren in der Praxis oft aufwendig und für den Operateur häufig zu unpräzis. Material und Methode: Seit Oktober 2004 haben wir an unserer Klinik 15 Fälle von nicht palpablen histologisch verifizierten Mammakarzinomen oder DCIS mit der ROLL-Methode markiert und operiert. Bei 11 Patientinnen wurde gleichzeitig eine Sentinel-Lymphknotenbiopsie durchgeführt. Hierbei erfolgte die radioaktive Markierung des nicht palpablen Befundes 1 Tag präoperativ interdisziplinär zwischen Radiologie und Nuklearmedizin. Die Applikation von MAASOL erfolgte sonographisch oder stereotaktisch gesteuert, je nach Darstellbarkeit des Befundes. Intraoperativ wurde eine Gamma-Sonde verwendet, um den Befund zu lokalisieren und die Resektionsgrenzen zu definieren. Ergebnisse: Alle Läsionen konnten präoperativ sicher markiert werden, intraoperativ konnten diese problemlos lokalisiert und exzidiert werden. Auch bei den Patientinnen mit gleichzeitiger Sentinel-Lymphknotenbiopsie war die Identifikation der Läsion gut möglich. Bei 3 Patientinnen war eine Nachresektion aufgrund ungenügender Resektionsränder nötig. Schlussfolgerung: ROLL ist eine präzise Methode um nicht palpable Brustläsionen präoperativ zu markieren. Die Methode, kann gleichzeitig mit der Sentinel-Lymphknotenbiopsie angewandt werden. Die Möglichkeit, den Befund intraoperativ immer wieder lokalisieren zu können, ist eine wesentliche Erleichterung für den Operateur.

\section{P VI/69 Senologie/Endokrinologie NuvaRing ${ }^{\circledR}$ verbessert die Zykluskontrolle bei Frauen in der Schweiz}

Merki-Feld G.S. ${ }^{\text {a }}$, Hund M. ${ }^{\text {b }}$

${ }^{a}$ Klinik für Endokrinologie, Departement Frauenheilkunde Universitätsspital Zürich, borganon AG, Pfäffikon

Einführung: Ein grosser Teil der Frauen in der Schweiz verhütet jahrelang mit oralen Kontrazeptiva. Besonders bei längerfristigem Gebrauch empfinden viele Pillenanwenderinnen es als unangenehm, täglich an die Verhütung denken zu müssen. Dies führt unter anderem zur Reduktion der Compliance und damit auch der Verhütungssicherheit. Mit NuvaRing ${ }^{\circledR}$, einem kontrazeptiven Vaginalring, der über 3 Wochen $15 \mu \mathrm{g}$ Ethinylestradiol (EE) und $120 \mu \mathrm{g}$ Etonogestrel pro Tag freisetzt, wurde eine neue einfach anzuwendende Verhütungsmethode eingeführt. In einem Praxiserfahrungsbericht (PEB) wurden Informationen über Zykluskontrolle, Akzeptanz und Verträglichkeit des NuvaRing ${ }^{\circledR}$ bei Anwenderinnen gesammelt. Methode: Die Daten des PEB wurden durch GynäkologInnen von Oktober 2003 bis Dezember 2004 in der Schweiz erhoben. Frauen, die eine Kontrazeption wünschten und bei denen keine Kontraindikation für die Anwendung von NuvaRing $^{\circledR}$ vorlag (gemäss Fachinformation), konnten teilnehmen. Ziel war die Anwendung von NuvaRing ${ }^{\circledR}$ in ungefähr 6 aufeinanderfolgenden Zyklen. Jeder Zyklus schloss 3 Wochen Ringanwendung, gefolgt von einer ringfreien Woche, ein. Informationen hinsichtlich Zykluskontrolle, Blutungsmuster und Dysmenorrhoe wurden bei der Eingangsuntersuchung und bei der Kontrolle gesammelt. Ergebnisse: 1487 Teilnehmerinnen wandten NuvaRing ${ }^{\circledR}$ für die Beobachtungsdauer von 4-7 Monaten an. 818 Frauen kamen aus verschiedenen Gründen früher zur Kontrolle und 267 später (k.A. für 70). Das mittlere Alter der Teilnehmerinnen war 27,6 Jahre ( \pm 7 Jahre). Bei der Eingangsuntersuchung hatten $84,2 \%$ der Teilnehmerinnen einen regelmässigen Zyklus, bei der Kontrolluntersuchung waren es $98,1 \%$. Die Anzahl der Blutungstage nahm unter der Anwendung von NuvaRing ${ }^{\circledR}$ von der Eingangsuntersuchung bis zur Kontrolle nach 4-7 Monaten ab (Tabelle 1).
196

Gynäkol Geburtshilfliche Rundsch 2005;45:165-221
Abstracts, gynécologie suisse, Lugano, 2005 


\begin{tabular}{llllrr}
\hline & \multicolumn{7}{l}{ Anzahl Blutungstage } \\
\cline { 2 - 6 } & $2-3$ & $4-5$ & $5-6$ & $6-8$ & $8-12$ \\
\hline Eingangsuntersuchung, \% Frauen & 15,9 & 32,5 & 29,9 & 16,6 & 2,5 \\
Kontrolle, \% Frauen & 29,2 & 41,6 & 21,4 & 5,9 & 0,8 \\
\hline
\end{tabular}

Anzahl Blutungstage bei Eingangsuntersuchung und Kontrolle bei 1,1\% bzw. 2,6\% der Frauen $<2$ Tage oder $>12$ Tage.

Eingangs bezeichneten 26,6\% der Teilnehmerinnen ihre Menstruationsblutung als leicht. Während der Anteil an Frauen mit leichter Blutung unter NuvaRing ${ }^{\circledR}$ auf $46,1 \%$ anstieg, sank der Anteil an Frauen mit mittleren und starken Blutungen um 26,6\%. Während 26,3\% der Frauen bei der Eingangsuntersuchung eine Dysmenorrhoe angaben, waren es nur noch 10,6\% Frauen bei der Kontrolle unter NuvaRing ${ }^{\circledR}$. Die Mehrheit der Teilnehmerinnen $(93,2 \%)$ war entweder zufrieden oder sehr zufrieden mit NuvaRing $^{\circledR}$. 86,7\% wollten NuvaRing ${ }^{\circledR}$ nach Ende des PEB weiter verwenden. Unerwünschte Arzneimittelwirkungen traten bei 13,7\% der Frauen mit einer Anwendungsdauer von 4-7 Monaten auf. Schlussfolgerung: Diese Ergebnisse zeigen, dass NuvaRing ${ }^{\circledR}$ in der täglichen Anwendung die Zykluskontrolle hinsichtlich Blutungsdauer, Blutungsintensität und Dysmenorrhoe verbesserte.

\section{P VII/70 Komplikationen in der Geburtshilfe Sexualité des femmes 18 ans après un accouchement}

Otero M., Bianchi-Demicheli F., Floris L., Sangalli M.R., Weil A., Boulvain M., Irion O., Faltin D.L.

Départements de Gynécologie-Obstétrique et de Psychiatrie, Hôpitaux Universitaires de Genève

Introduction: Nous avons étudié l'association entre une déchirure sphinctérienne suite à un accouchement et la fonction sexuelle à long terme. Méthodes: Nous avons identifié 445 femmes avec atteinte sphinctérienne ayant accouché en 1982-1983 (445/4569 naissances, 97\%) et une cohorte de 445 femmes sans déchirure du sphincter. Les primipares ayant plus de lésions, une sélection a été faite pour avoir le même nombre de primipares et de multipares dans les deux groupes. Un questionnaire leur a été envoyé comportant 19 questions sur 6 domaines: le désir, l'excitation, la lubrification, l'orgasme, la satisfaction et la douleur. Résultats: Sur 890 femmes interrogées, 453 (51\%) ont complété les questions sur la fonction sexuelle. 180/223 (81\%) femmes avec atteinte sphinctérienne avaient une activité sexuelle et 179/230 (78\%) contrôles (risk ratio $1.0,95 \% \mathrm{CI} 0,9-1,1, \mathrm{p}=0,45)$. Il n'y avait pas de différence entre les groupes pour les questions sexuelles sauf une concernant la lubrification (29\% des femmes avec lésion sphinctérienne et $20 \%$ des contrôles avaient une lubrification durant les rapports moins de la moitié du temps, risk ratio $1,4, \mathrm{CI} 95 \% 1,0-2,1, \mathrm{p}=0,03)$. Les scores de fonction sexuelle étaient similaires entre les deux groupes (median 29,9, Wilcoxon rank-sum test $\mathrm{p}=0,38$ ). Des perturbations de la fonction sexuelle étaient signalées par $26 \%$ des femmes avec atteinte du sphincter et $25 \%$ des contrôles (risk ratio 1,1, 95\% CI $0,7-1,5, p=0,74$ ). Les réponses de 16 femmes ayant accouché par césarienne étaient identiques aux autres. L'épisiotomie, l'accouchement instrumenté, un poids fotal élevé n'étaient pas associés avec une perturbation de la fonction sexuelle. Conclusion: Nous n'avons pas découvert d'augmentation du risque de perturbation de la fonction sexuelle 18 ans après un accouchement traumatique.

Fonds National Suisse de la Recherche Scientifique no 3255907.98 et 3200-064056.00.

\section{P VII/71 Komplikationen in der Geburtshilfe Invasive Blasenmole bei einer 48-jährigen Patientin}

\section{Brancatelli G., Kleeb B., Beck G.}

Frauenklinik, Kantonsspital Olten

Einführung: Die invasive Blasenmole gehört zu den villösen gestationsbedingten Trophoblasterkrankungen (GTE). Mit einer Inzidenz von 1:15 000 Schwangerschaften ist sie selten und tritt häufiger bei Schwangerschaften sehr junger oder älterer Patientinnen auf. Aus unbekannten Gründen ist die Inzidenz in asiatischen Ländern fast 10mal höher. Wir stellen einen Fall vor, der eine alte Asiatin mit grossem Uterus myomatosus betrifft. Fallvorstellung: Eine 48-jährige Mongolin, Gravida IV, Para III wurde uns in der 82/7 Amenorrhoewoche wegen rezidivierenden Schmierblutungen zugewiesen. Der SS-Test war positiv, sonographisch konnten weder eine intra- noch eine extrauterine Schwangerschaft nachgewiesen werden, dafür war der Uterus bis auf Nabelhöhe vergrössert und sehr derb. In der Sonographie stellte sich ein bläschenartiger, vaskularisierter intrauteriner Tumor dar, der gegen die Vorderwand nicht abzugrenzen war. Der Beta-HCG-Wert betrug 265732 IE/l. Eine MRI-Untersuchung bestätigte die Verdachtsdiagnose eines Uterus multimyomatosus mit intracavitärem Tumor. Die systemischen Abklärungen ergaben keine Anhaltspunkte für Metastasen (CT Schädel/Thorax, MRI Abdomen, Zysto-Rektoskopie). Da es sich nach den verschiedenen Scores (WHO, FIGO, Newlands Score) um eine bezüglich Prognose high-risk-Situation handelte und ein Chorionkarzinom auch möglich war, haben wir die Patientin zur weiteren Therapie in ein universitäres Zentrum überwiesen. Einer Methotrexattherapie folgte am gleichen Tag die Hysterektomie. Histologisch handelte es sich um eine invasive Blasenmole mit herdförmiger starker Proliferation von atypischen Trophoblasten in einem Uterus multimyomatosus. 9 Monate nach dem Eingriff ist die Patientin beschwerdefrei mit einem Beta-HCG $<2$ IE/l. Diskussion: Ein Blick in die Literatur zeigt, dass ein high -risk-Trophoblasttumor bei einer Patientin mit offener Familienplanung zu einer der vielen vorgeschlagenen Chemotherapie-Kombinationen (EMA-CO, BEP, VIP etc.) führt und in ca 30\% der Fälle erfolglos (Persistenz oder Rezidiv) bleibt. In unserem Fall wurde trotz high-risk-Situation bei abgeschlossener Familienplanung, Begleitdiagnose und fehlendem Metastasennachweis der Weg einer operativen Behandlung gewählt. Die Pflicht ist es während 2 Jahren, zu Beginn wöchentlich und anschliessend monatlich, den Hormontiter im Auge zu behalten.

\section{P VII/72 Komplikationen in der Geburtshilfe Schwangerschaft nach Endometriumablation mittels Thermachoice ${ }^{\mathrm{TM}}$ - Schwere Komplikationen in der Plazentarperiode}

Grabbert M., Vogt H.P.

Frauenklinik, Spital Region Oberaargau, Langenthal

Einführung: Die Endometriumablation mittels Therma Choice $^{\mathrm{TM}}$ als Therapieverfahren bei Blutungsstörungen ist bekanntlich keine Methode zur Antikonzeption. Dies wird den Patientin- 
nen vor einem solchen Eingriff mitgeteilt. Die Rate an Schwangerschaften unter ThermaChoice ${ }^{\mathrm{TM}}$ wird in der Literatur mit rund 0,7\% angegeben. Es wurden neben komplikationslosen Schwangerschaftsverläufen aufgrund der Endometriumdestruktion Plazentationsstörungen und schlechte fetal outcomes beschrieben. Kasuistik: 2 Jahre nach ThermaChoice ${ }^{\mathrm{TM}}$ Endometriumablation wegen Hypermenorrhoe Eintreten einer erwünschten Gravidität bei 35jähriger III Gravida II Para. Abortus imminens-Symptomatik in der 19. SSW. Spontangeburt eines Mädchens in der $37+4$ SSW mit postpartaler Plazentaretention und beginnender Inversio uteri. Nach der manuellen Plazentalösung kam es zu einer atonischen Blutung mit geschätztem Blutverlust von 3000 ml. 14 Tage später Wiedereintritt wegen beginnender Sepsis. Bei Hinweis auf Restplazenta Versuch einer Nachcurettage. Bei intraoperativ lebensbedrohlicher Blutung erfolgte die notfallmässige abdominale Hysterektomie. Unkomplizierter postoperativer Verlauf. Histologische Diagnose einer Plazenta accreta. Schlussfolgerungen: Obwohl in der Literatur komplikationslose Schwangerschaften nach Endometriumablation mittels ThermaChoice ${ }^{\mathrm{TM}}$ beschrieben werden, ist die Nidationsstörung der Plazenta nach diesem Eingriff eine schwere Komplikation, die nicht ausser Acht gelassen werden darf. Vor der Durchführung während der reproduktiven Phase muss die Patientin über zusätzlich nötige Verhütungsmassnahmen und im Falle einer eintretenden Schwangerschaft über das Risiko einer Nidationsstörung der Plazenta aufgeklärt werden. Wegen zu erwartender schwerwiegender Probleme in der Plazentarperiode ist es ratsam, bei Status nach Endometriumablation auf eine Spontangeburt zu verzichten und eine primäre Sectio durchzuführen. Mit einer Sectio-Hysterektomie muss in jedem Fall gerechnet werden.

\section{P VII/73 Komplikationen in der Geburtshilfe "Giant» Paravaginalzyste - (K)Ein Geburtshindernis?}

\section{Breymann C. ${ }^{\mathrm{a}, \mathrm{b}}$, Burkhardt T.}

${ }^{a}$ Klinik für Geburtshilfe, Departement Frauenheilkunde, Universitätsspital Zürich, ${ }^{b}$ Gynäkologie-Geburtshilfe-Seefeld (GGS), Zürich

Einleitung: Paravaginalzysten entstehen am häufigsten aus persistierenden Anteilen des Müllerschen Ganges. Seltener sind Paravaginalzysten aufgrund persistierender Reste des Urnierenganges (Wolffscher Gang), sogenannte Gartner-Zysten. In Abhängigkeit von der Grösse verhalten sich die Zysten oft klinisch stumm und sind häufig Zufallsbefunde. Fallvorstellung: Wir dokumentieren den Befund und Verlauf bei einer 32-jährigen Primigravida/Primipara, bei welcher bereits im Jahr 2000 eine Paravaginalzyste marsupialisiert wurde. Nunmehr stellte sich die Patientin mit einem grossen Zystenrezidiv in der Schwangerschaft vor, subjektiv ohne Beschwerden. Klinisch erschien der Befund weich und elastisch, den Geburtskanal nicht obstruierend. Zur Abwägung eines möglichen Geburtshindernisses bei vaginaler Geburt wurden bildgebende Verfahren eingesetzt. Methodik/Verlauf: Bildgebende Darstellungen erfolgten mittels transvaginalem Ultraschall (2D \& 3D Technik) und MRI des Beckens (native Schichtaufnahmen in drei Ebenen in verschiedenen Echosequenzen). Ausserhalb des kleinen Beckens zeigte sich paravaginal und pararectal in der Fossa ischiorectalis eine zystische Raumforderung von $11 \times 6 \times 6 \mathrm{~cm}$ Durchmesser mit einer eiförmigen soliden Raumforderung von knapp $7 \times 5 \times 5 \mathrm{~cm}$ Durchmesser. Der Aufhängeapparat der Beckenorgane wurde durch die Raumforderung leicht nach links verlagert. Die Beckenmasse waren im MRI normal. Eine konklusive Meinung über ein mögliches Geburtshindernis wurde von den Ra- diologen nicht abgegeben. Aufgrund der klinischen Befunde (s.o.) wurde eine vaginale Geburt angestrebt, diese erfolgte bis auf einen DR III. Grades und erhöhten Blutverlust erfolgreich. Schlussfolgerung: Paravaginalzysten dieser Grösse sind selten, eine gute Beratung der Patientin nicht einfach. In diesem Fall war der klinische Verlauf komplikationslos und die Spontangeburt möglich. Auch sehr grosse Paravaginalzysten stellen somit primär keine Kontraindikation für eine vaginale Geburt dar.

\section{P VII/74 Komplikationen in der Geburtshilfe Streptokokken A als Ursache einer septischen puerperalen Ovarialvenenthrombose}

Frolik $D .^{\mathrm{a}}$, Leitner $\mathrm{U}^{\mathrm{b}}$, Beer $P^{\mathrm{a}}{ }^{\mathrm{a}}$ Kaufmann $M .^{\mathrm{a}}$

${ }^{a}$ Klinik für Gynäkologie und Geburtshilfe,

${ }^{b}$ Institut für Radiologie, Spital Bülach

Fallpräsentation, Anamnese: 28-jährige IP/IIIG mit Chlamydiencervicitis in der 32. SSW mit vorz. Wehentätigkeit u. Portioreifung. Adalattokolyse u. Antibiotika-Gabe mit nachfolgend neg. Kontrollabstrich. Einleitung mit Oxytocin bei $41+4$ SSW. Spontangeburt Knabe 4350 g, DR II. Entlassung am 5. postpartalen Tag in gutem AZ. Klinik, Befunde, Therapie: (1) Kons. 7 Tage postpartal mit Fieber bis $40^{\circ} \mathrm{C}$ u. wässriger Diarrhoe bei blander körperlicher Untersuchung. Besserung auf Therapie mit Paracetamol u. Perenterol. (2) Kons. 12 Tage postp. mit trockenem Husten. Klinisch punktförmige Druckdolenz im li UB. Spekulumuntersuch unauffällig. Bimanuell kein Portioschiebeschmerz, Adnexbereich frei. VUS: Schmales Endom. 2 mm, unklare Raumforderung parauterin li, keine freie Flüssigkeit. (3) Kons. 14 Tage postp. mit Diarrhoe, Fieber bis $39^{\circ} \mathrm{C} \mathrm{u}$. Schmerzen im li UB. Bei genauem Nachfragen Halsschmerzen. Klinisch gespanntes Abd. CT-Abd.: Ödematöses Ovar paraut. li $5 \times 6 \mathrm{~cm}$. Thrombosierte li Ov.vene als tubuläre Struktur retroperitoneal. Keine AP für Divertikulitis. Stuhluntersuch: Keine Lc nachweisbar. Labor: CRP 179 mg/l, Lc 30 000/ $\mu 1$ mit Li-verschiebung. Blutkultur: Strept. Gruppe A. Therapie: Antikoagulation mit Fraxiforte u. überlappend Marcoumar. Iv Antibiotika Zinacef u. Dalacin. Resistenzgerecht weiter mit Zinat per os. Abgestillt auf Wunsch mit Dostinex. Entlassung am 6. Hospitalisationstag in deutlich gebessertem AZ. Labor: CRP

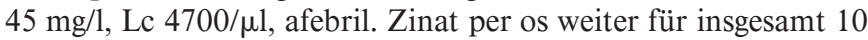
Tage. Marcoumar für 6 Wochen. Diskussion: Strept. A sind bekannte Erreger der puerperalen Sepsis. In Einzelfällen wurden sie als Ursache einer Ovarialvenenthrombose beschrieben. Typisch sind die initial fehlenden u. später unspezifischen Symptome, welche aber schnell in einen septischen Schock mit DIC übergehen können. In 90\% Lokalisation der Thrombose in der re Ovarialvene. Hier li-seitige Lokalisation ohne klin. Zeichen einer Endomyometritis, Vd.a. pharyngitische Streuung. Schlussfolgerung: Die septische pelvine Thrombophlebitis ist eine gefährliche Komplikation mit hoher Morbidität und Mortalität. Diagnostische Sicherung erfolgt durch Sonografie, CT oder MRT. Die sofortige Therapie mit Antikoagulation u. Breitbandantibiotika verhindert effizient die Embolisation u. septische Streuung.
Abstracts, gynécologie suisse, Lugano, 2005 


\section{P VII/75 Komplikationen in der Geburtshilfe \\ Plicature utérine de B-Lynch en cas \\ d'hémorragie aiguë du post-partum: \\ a propos d'un cas}

Département de Gynécologie-Obstétrique, Centre Hospitalier

Introduction: L'hémorragie du post-partum reste une complication grave intéressant 4-5\% des accouchements. En cas d'échec de mesures obstétricales habituelles (révision utérine, examen de la filière génitale, perfusion d' oxytocine ou de prostaglandines), une prise en charge plus invasive est nécessaire. Outre l'embolisation des artères utérines et la ligature chirurgicale étagée de la vascularisation utérine, la technique de B-Lynch a été décrite comme traitement de l'hémorragie en cas d'atonie utérine. Matériel et méthodes: Une patiente $2 \mathrm{G} / 0 \mathrm{P}$ de 32 ans à 38 semaines de grossesse est admise pour rupture prématurée des membranes. Début d'une provocation par Cytotec (50 $\mu \mathrm{g}$ intra-vaginal) $24 \mathrm{~h}$ après la rupture. Pose de péridurale. Souffrance foetale à $8 \mathrm{~cm}$ de dilatation nécessitant une césarienne en urgence. Naissance d'une fille de $2720 \mathrm{~g}$ pour 47,5 cm, Apgar 9-10-10, $\mathrm{pHa} 7.04$, pHv 7.05. Après la délivrance du placenta et de la fermeture de l'utérotomie persiste une atonie utérine sévère s'accompagnant d'une hémorragie très importante. Malgré un massage utérin et l'administration d'oxytociques iv, de Méthergin et de Nalador intra-myométral, associés à $1000 \mu \mathrm{g}$ de Cytotec intra-rectal, l'utérus ne se contracte Lynch, combinée à une irrigation intra-utérine de prostaglandines. Résultats: Tarissement immédiat du saignement vaginal une fois la «bretelle» serrée. L'irrigation intra-cavitaire a été maintenue pendant $12 \mathrm{~h}$ à un débit de $60 \mathrm{ml} / \mathrm{h}$. Les pertes sanguines ont été normales ensuite. La patiente a perdu environ $1800 \mathrm{ml}$ de sang et l'hémoglobine a chuté de 110 à $58 \mathrm{~g} / 1$ sans nécessité de transfusion. Le retour à domicile a eu lieu au sixième jour post-opératoire. Conclusion: La plicature utérine mimant les bretelles d'un sac à dos selon la technique de B-Lynch est un moyen simple et efficace, permettant de surseoir à une embolisation des artères utérines (si celle-ci n'est pas réalisable) ou encore à une hystérectomie.

\section{P VII/76 Komplikationen in der Geburtshilfe Plazentainvasionsstörungen: Eine monozentrische Studie}

Bencaiova G., Burkhardt T., Beinder E.

Klinik für Geburtshilfe, Departement Frauenheilkunde, Universitätsspital Zürich

Einführung: Im Licht der erhöhten Anzahl von Curettagen und vorherigen Gebärmutteroperationen nehmen die Plazentainvasionsstörungen an klinischer Bedeutung zu. Die pränatale Erkennung von Plazentainvasionsstörungen, die adäquate Vorbereitung für massive Bluttransfusionen, erfahrene chirurgische und anästhesiologische Teams, intensive Überwachung und sorgfältige Planung können den Blutverlust während der Geburt und das Risiko von maternalen und neonatalen Komplikationen reduzieren. Material und Methode: Eine retrospektive Studie von den Krankengeschichten aller Geburten zwischen 1999-2003 im Universitätsspital Zürich wurde durchgeführt und die Häufigkeit, Risikofaktoren und Geburtsmodi bei Patientinnen mit Plazentainvasionsstörungen wurden untersucht. Ergebnisse: 31 Frauen mit
Warkus T., Diaz M., Hohlfeld P., Delaloye J.-F.

Universitaire Vaudois, Lausanne pas. Nous effectuons une plicature utérine selon la technique de B-

einer Plazentainvasionsstörung wurden identifiziert. Diese Anzahl entspricht $0,31 \%$ aller Geburten in der Fünfjahresperiode (31/10181). Wir haben keinen Unterschied betreffend Alter der Mütter, Gravidität und Parität zwischen Patientinnen und gesunder Kontrollgruppe festgestellt. Signifikante Risikofaktoren für eine Plazentainvasionsstörung sind vorgängige Curettagen $(\mathrm{OR}=$ 19,3; 95\% CI 11,6-32,3), Gebärmutteroperationen (ausser Kaiserschnitt), (OR = 49,6; 95\% CI 24,3-102,3) und gleichzeitige Placenta praevia $(\mathrm{OR}=16,1 ; 95 \%$ CI 4,7-43,7). Vierzehn Patientinnen wurden spontan entbunden. Bei einer Patientin wurde wegen unkontrollierter Blutung eine totale Hysterektomie durchgeführt und die Placenta increta wurde durch die histologische Untersuchung bestätigt. Siebzehn Frauen wurden durch Kaiserschnitt entbunden, bei vier musste eine totale Hysterektomie und bei einer Patientin eine Gebärmutterrekonstruktion nach der teilweisen Entfernung der vorderen Gebärmutterwand durchgeführt werden. Wir haben in unserer Studiengruppe keinen mütterlichen Todesfall, aber aufgrund extremer Frühgeburtlichkeit zwei neonatale Todesfälle beobachten müssen. Durch eine pränatale Ultraschalluntersuchung bestand bei 10 Patientinnen der Verdacht auf eine Plazentainvasionsstörung. Schlussfolgerung: Der wichtigste Risikofaktor für eine Plazentainvasionsstörung ist ein vorheriger Gebärmuttereingriff. Die pränatale Ultraschalluntersuchung bei Risikopatientinnen ermöglicht häufig die Verdachtsdiagnose und damit die Optimierung des peripartalen Managements.

\section{P VII/77 Komplikationen in der Geburtshilfe Wiederholte arterielle Embolisation der Arteriae uterinae nach Sectio und Resectio caesarea bei Plazenta praevia totalis}

Schrack V., Schoch E., Zollikofer C.L., Hess Th., Müller R.C.

Frauenklinik, Kantonsspital Winterthur

Einführung: 40-jährige IIG IIP wurde 2002 und 2004 per Sectio caesarea bei Plazenta praevia totalis entbunden. Jedes Mal postoperativ massiver Blutverlust (2002: $3500 \mathrm{ml}, 2004: 5000 \mathrm{ml}$ ) ohne erfolgreiche Blutstillung trotz aller gängigen Massnahmen. Deshalb jeweils Durchführung einer arteriellen Embolisation der Arteriae uterinae und Vermeidung einer Hysterektomie. Material und Methoden: In Zusammenarbeit mit Gefässzentrum Winterthur Verfahrenserläuterung der wiederholten zielgerechten arteriellen Embolisation mit Gelfoam-Artikel. Zusätzlich bildgebender Vergleich und Ergebnisbeurteilung. Ausserdem Erwähnung der hierfür nötigen medizinischen Infrastruktur. Ergebnisse: Gute Resultate nach mehrmaliger Embolisation der nach zervikal und uterin führenden Gefässäste an der Arteria illiaca interna. Im Vergleich zur Vorunteruchung von 2002 weitgehender Verschluss der Arteriae uterinae, aber es finden sich erneut zervikale Gefässe, welche aus dem selben Hauptstamm wie die Arterie uterina stammen. Spezielles Eingehen in dieses Gefäss und Embolisation mittels Gelfoam-Artikel mit vollständiger Stase. Die Kontrollangiographie zeigte den gewünschten Verschluss der behandelten Gefässe und analog zur Gegenseite feine Kollateralgefässe entlang der Beckenwand. Die Behandlung wurde seitens der Patientin sehr gut vertragen. Schlussfolgerung: Die Embolisation der uterinen Gefässe scheint eine alternative Therapiemöglichkeit zur Behebung von operativ oder medikamentös nicht stillbaren Blutungen zu sein und bringt eine eindrückliche Gefässveränderung mit sich. Die Zukunft wird zeigen, ob dieser Therapieansatz weiter an Bedeutung gewinnt. 


\section{P VII/78 Komplikationen in der Geburtshilfe Mehr Resectiones, mehr Komplikationen? Analyse geburtshilflicher Parameter primärer Resectiones im Vergleich zu Sectiones}

Manegold G., Humburg J., Hösli l., Holzgreve W.

Universitäts-Frauenklinik, Universitätsspital Basel

Einführung: Mit der steigenden Anzahl der Wunschsectiones steigt auch die Anzahl der Resectiones. Der Frage, ob dieser Anstieg von Resectiones auch mit einem vermehrten Auftreten von Komplikationen, die mit der Resectio einhergehen, verbunden sind, wollten wir am eigenen Patientengut nachgehen. Material und Methoden: Wir führten eine retrospektive Analyse durch, bei welcher 105 konsekutive Sectiones (Beckenendlage, Wunsch, hoher Gradstand) mit 105 konsekutiven Resectiones verglichen wurden. Die Gruppen unterschieden sich hinsichtlich Geburtsgewicht und Schwangerschaftsalter nicht. Folgende Parameter wurden untersucht: intraoperativer Blutverlust, Operationsdauer, Verwachsungen, erschwerte Kindesentwicklung, Apgarwerte, $\mathrm{pH}$-Werte, postoperativer Hämoglobinwert, Hospitalisationsdauer und Wundheilungsstörungen. Ergebnisse: Überraschenderweise unterschieden sich die beiden Gruppen der Sectiones und der primären Resectiones auf Wunsch praktisch nicht. Nur im Hinblick auf den postoperativen Hämoglobinwert war ein signifikanter Unterschied vorhanden, wobei wider Erwarten das Hämoglobin in der Gruppe der Resectiones höher war. Schlussfolgerung: Resectiones führten in unserem Patientengut bei 105 konsekutiven Operationen nicht zu vermehrten Komplikationen im Vergleich zu 105 konsekutiven Sectiones. Einziger Unterschied war der tiefere postoperative Hämoglobinwert in der Gruppe der Sectiones. Eine mögliche Ursache könnte sein, dass eine Resectio in unserem Haus in der Regel durch einen erfahreneren Operateur durchgeführt wird, der sich der möglichen Komplikationen bewusst ist und diese umsichtig zu vermeiden sucht. Denkbar wäre ebenfalls, dass eine Operation im Narbengewebe aufgrund der schlechteren Vaskularisation tendenziell weniger zur Blutung neigt als das normale Gewebe.

\section{P VII/79 Komplikationen in der Geburtshilfe Mütterlicher Todesfall durch Fruchtwasserembolie}

Hagen B.D. ${ }^{\mathrm{a}}$, Todesco M. ${ }^{\mathrm{a}}$, Stallmach T. ${ }^{\mathrm{b}}$, Zimmermann . $^{\mathrm{a}}$

${ }^{a}$ Klinik für Geburtshilfe, Departement Frauenheilkunde, Universitätsspital Zürich, 'Institut für Klinische Pathologie, Universitätsspital Zürich

Die Fruchtwasserembolie ist eine seltene, zumeist leider jedoch tödliche Komplikation der Geburt, die für 10\% der mütterlichen Sterblichkeit verantwortlich ist. Die Inzidenz ist bis zu 1 in 20000 Geburten und Mortalitätsrate ist zw. 80 und 90\%, mit fetalem Tod in ca. 40\%. Die Diagnose wird aufgrund der Klinik gestellt: akutes Herzversagen, akutes Lungenversagen und/oder DIC unter der Geburt oder im frühen Wochenbett. Der Nachweis fetaler Zellen im mütterlichen Gewebe stützt die Diagnose, beweist sie jedoch nicht. Wir stellen den Fall einer 34-jährigen IG IP vor, welche bei fetaler Bradykardie und gleichzeitiger Bewusstlosigkeit und Bradykardie mittels Forzeps entbunden wurde. Nach vollständiger Entwicklung der Plazenta kam es zur massiven Nachblutung ohne Koagelbildung und ohne Hinweis für Atonie. Nach Ausschöpfen aller konservativen Möglichkeiten wurde die Hysterektomie durchgeführt. Intraoperativ musste sie zweimal mechanisch reanimiert werden. Es wurden Massentransfusionen gegeben und sie wurde katechola- minpflichtig auf die Intensivstation verlegt. In der Folge kam es zu einem massiven Lungenödem und akuten Nierenversagen. Während der Hämofiltration ereignete sich erneut ein Kreislaufstillstand, welcher nicht mehr zu beheben war. Eine Obduktion wurde durchgeführt und die Diagnose der Fruchtwasserembolie erhärtet. Es werden die pathologischen Befunde dargestellt und im Hinblick auf die Pathophysiologie diskutiert.

\section{P VII/79.1 Komplikationen in der Geburtshilfe \\ Rupture utérine, pendant le travail, d'un uterus non cicatriciel, après application d'une pression fundique}

Vuilliomenet A., Bracher Ph., Chabloz P.

Service de Gynécologie-Obstétrique, Hôpital Pourtalès, Neuchâtel

Introduction: Les ruptures spontanées sur utérus non cicatriciel sont rares, avec une incidence qui varie selon les auteurs entre $1 / 8000$ à 1/16 000 accouchements. Nous rapportons ici un cas de rupture spontanée pendant le travail. Observation: Patiente de 35 ans, 2 gestes, 1 pare, qui a été admise dans notre service à 40 SA, à dilatation complète. Après rupture artificielle des membranes et 10 min de poussées actives ainsi que 2 Kristellers en raison d'une souffrance fœtale aiguë, naissance d'une fille pesant $3800 \mathrm{~g}$ et mesurant $50 \mathrm{~cm}$. Vingt min après la délivrance qui fut sans particularité, la patiente a décrit une vive douleur continue au niveau de l'hypochondre droit. L'examen clinique associé aux examens complémentaires faisant suspecter une rupture utérine, une laparotomie médiane a été pratiquée en urgence. Celle-ci a confirmé la rupture longitudinale latéro-isthmique droite sur $4 \mathrm{~cm}$. L'utérus a été suturé. Il n'y a pas eu d'autres complications et les suites post-opératoires ont été simples. Discussion: On connaît bien les principaux facteurs de risque associés à la rupture utérine spontanée sur utérus non cicatriciel: un traumatisme abdominal, les manœuvres obstétricales, la grande multiparité, les malformations utérines, la corticothérapie chronique, l'utilisation inadéquate d'ocytocique ainsi que les antécédents de curetage. Cependant, on ne possède que peu de données quant aux risques de rupture liés aux Kristellers. En effet, elles ne sont pas publiées pour des raisons médicolégales même si certaines publications suggèrent que les risques existent. Une faiblesse dans la musculature utérine, consécutive aux premiers Kristellers pratiqués lors du premier accouchement pour non-progression de la présentation associée aux pressions fundiques et à la force maternelle lors du deuxième accouchement, peuvent expliquer, dans ce cas précis, la rupture spontanée. Conclusion: Cette observation permet de rappeler que l'absence d'antécédent d'utérus cicatriciel ne devrait pas éliminer l'évocation du diagnostic de rupture utérine. Une détection rapide et une intervention chirurgicale immédiate sont les points principaux de la prise en charge de cette complication obstétricale rare et d'autant plus menaçante.

\section{P VIII/80 Geburtshilfe: Fallberichte Adénome hypophysaire et grossesse}

Hachemane K., Cavenaghi E., Tolck P.H.

Hôpital Communal, La Chaux-de-Fonds

Introduction: L'hypophyse augmente de manière physiologique durant la grossesse en raison de l'augmentation du nombre et de la taille des cellules à prolactine. Cependant, en présence de 
troubles hormonaux ou de céphalées persistantes, il faut penser à l'adénome hypophysaire. Présentation du cas: Patiente de 25 ans, en bonne santé habituelle, secondigeste primipare, avec antécédent de forceps en 2001. Hospitalisation à deux reprises à 34 puis 35 semaines d'aménorrhée en raison de céphalées persistantes; bilans de gestose normaux. Mise en évidence d'une hémianopsie bitemporale à l'examen clinique. L'IRM met en évidence un probable macro-adénome hypophysaire de $20 \mathrm{~mm}$ de plus grand axe, avec compression sur le chiasma optique. Provocation de l'accouchement puis césarienne à 36 semaines pour souffrance foetale aiguë à $4 \mathrm{~cm}$ de dilatation. Transfert en milieu universitaire à $\mathrm{J} 7$ postpartum. Opération à J9: vidange de l'hypophyse par voie transsphénoïdale. Diagnostic anatomo-pathologique: Adénome hypophysaire ayant subi un accident évolutif de type ischémique. Bilan endocrinien strictement normal, prolactine plutôt basse $(9,4)$. Suites simples, diabète insipide traité par desmopressine. Discussion: Le diagnostic des masses sellaires se pose soit lors de troubles visuels et/ou de céphalées, soit en présence de troubles hormonaux, soit lors d'un CT ou d'une IRM pour une autre raison. La plupart sont des adénomes, sécrétants dans 60 à $70 \%$ des cas. Un accident évolutif dans l'adénome sera la cause de céphalées soudaines avec parfois diplopie. Le diagnostic est confirmé par IRM. La prise en charge du microadénome diffère de celle du macroadénome, car le premier n'augmente que peu durant la grossesse. Le macroadénome se traite par chirurgie versus traitement symptomatique, avec éventuelle irradiation par $45 \mathrm{~Gy}$. Le diabète insipide est une complication classique après traitement chirurgical. Il se traite par administration de desmopressine qui n'est pas contre-indiquée durant la grossesse. Conclusion: L'adénome hypophysaire représente une cause non rare de céphalées résistantes durant la grossesse et son traitement est le plus souvent chirurgical, au deuxième trimestre ou après provocation de l'accouchement si le terme le permet.

\section{P VIII/81 Geburtshilfe: Fallberichte Geburtserlebnis des Vaters in der alternativen Geburtsmedizin}

Rautenberg O., Zimmermann K., Eberhard J., Geissbühler V.

Frauenklinik, Kantonsspital Frauenfeld

Einführung: Die Anwesenheit des Vaters und seine Übernahme von Betreuungsfunktionen bei der Geburt sind heute eine Selbstverständlichkeit. Bisher wurde vor allem das mütterliche Geburtserlebnis untersucht, dasjenige des Vaters blieb jedoch wenig berücksichtigt. In der vorliegenden Arbeit wird das Geburtserlebnis des Vaters nach Spontangeburten in Kopflage dargestellt und die drei häufigsten Gebärmethoden - Wasser-, Maiahocker- und Bettgeburten - miteinander verglichen. Material und Methoden: In einer prospektiven Beobachtungsstudie wurden im Zeitraum vom 1. Nov. 1991 bis 31. Okt. 2004 insgesamt 16785 Geburten erfasst. Im Rahmen dieser Studie konnten 10653 Väter nach ihrem Geburtserlebnis unmittelbar nach der Geburt und während der Wochenbettzeit befragt werden. Dies erfolgte mit Hilfe eines standardisierten Fragebogens und einer visuellen Analogskala (0$100 \mathrm{~mm}, 0=$ «traumhaft schön» $/ 100=$ «furchtbar schlimm»). Ausgewertet wurden die Antworten der Väter von spontanen Einlingsgeburten aus Kopflage, welche die vorgegebenen Fragen aus sprachlichen Gründen beantworten konnten. Dies waren $n=8441$ Väter, davon Bettgeburten 36\% $(\mathrm{n}=3020)$, Wassergeburten $48 \%$ $(\mathrm{n}=4080)$, Maiahockergeburten 9\% $(\mathrm{n}=790)$, andere Geburten $7 \%(\mathrm{n}=551)$. Ergebnisse: Befragt nach der Erinnerung an die Geburt (Mögliche Antworten: «sehr schön»/«schön»/«mässig»/ «schlimm»/«keine Meinung») zeigten Partner von Erst- wie von
Mehrgebärenden nach Wassergeburten im Wochenbett ein signifikant schöneres Geburtserlebnis im Vergleich zu Maiahocker- und Bettgeburten (94 vs. 90 vs. 84\%). Das schlimmste Geburtserlebnis mit 2,9\% zeigten Väter nach Bettgeburten. Diese Ergebnisse bestätigen sich bei der Geburtserlebnisquantifizierung mit der visuellen Analogskala (VAS 0-100 mm): Mittelwert nach Wassergeburt $21 \mathrm{~mm}$, Mittelwert nach Maiahockergeburt $26 \mathrm{~mm}$, Mittelwert nach Bettgeburt $28 \mathrm{~mm}$. Schlussfolgerung: Väter, welche Gebärende bei einer Wassergeburt begleiten und betreuen, erleben diese Gebärmethode als am schönsten, dies gilt für Väter von Erst- wie auch von Mehrgebärenden. Die gewählte Gebärmethode, hier die Wassergeburt, beeinflusst - wie bei der Mutter - das Geburtserlebnis positiv.

\section{P VIII/82 Geburtshilfe: Fallberichte \\ Eine aussergewöhnliche Ursache für eine Fülle von «häufigen Schwangerschaftskomplikationen "}

Dombi V.H. ${ }^{a}$, John H. ${ }^{\text {b }}$, Honegger Ch. ${ }^{a}$

${ }^{a}$ Klinik für Gynäkologie und Geburtshilfe, Spital Uster,

${ }^{b}$ Klinik und Poliklinik für Urologie, Universitätsspital Zürich

Einführung: Unterschenkelödeme, schwangerschaftsinduzierte Hypertonie, Gestationsdiabetes und psychische Dekompensation bei sozialen Problemen sind häufig diagnostizierte Schwangerschaftskomplikationen. Hier beschreiben wir eine Patientin mit sämtlichen Symptomen, deren Ursache auf eine einzige Erkrankung zurückzuführen ist. Material und Methode: Fallpräsentation einer 30j. IIP/IIIG, die bei 21 SSW wegen Polydypsie von 10 1/d und massiven Unterschenkelödemen zugewiesen wird. Es wird die Diagnose eines psychogenen Diabetes insipidus mit hypostatischen Unterschenkelödemen gestellt aufgrund der erhaltenen Urinkonzentrationsfähigkeit im Wasserrestriktionstest. Ein weiterer Befund ist eine seit kurzem bestehende Akne am Rücken. Nach Trinkverhaltenstraining erfolgt die Demissio. Bei $31 \mathrm{SSW}$ weist sich die Patientin zu wegen massiven Oedemen und Erschöpfung bei sozialen Problemen. Es findet sich eine Schwangerschaftshypertonie, die sich stabilisiert. Während der Hospitalisation wird ein insulinpflichtiger Gestationsdiabetes diagnostiziert und die Patientin eingestellt entlassen. Bei 33 SSW notfallmässige Hospitalisation wegen Überforderung im Umgang mit dem Diabetes und psychischer Dekompensation bei der Neudiagnose einer Borderline-Persönlichkeitsstörung. Bei 37 1/7 SSW wird die Patientin per sekundärer Sectio von einem lebensfrischen Knaben entbunden. Peripartal fallen massive Striae rubrae auf, weshalb aufgrund aller Symptome die Verdachtsdiagnose eines Cushing-Syndroms gestellt wird. Ergebnis: Urin-Cortisol-Spiegel, ACTH-Test, Hyperandrogenämie, Hypophysen-MRI und Abdomen-CT bestätigen einen Nebennieren-Tumor, der operativ enfernt wird. Histologisch findet sich ein $3,5 \mathrm{~cm}$ grosses Nebennierenadenom. Bisher sind erst 110 Fälle eines Cushing-Syndroms in der Schwangerschaft beschrieben, da im Allgemeinen die Fertilität eingeschränkt ist. Gegen Ende der Schwangerschaft findet sich hier das Vollbild eines Cushing-Syndroms mit allen klinischen Zeichen, die wir dokumentiert haben. Die zusätzliche Hyperandrogenämie lässt auf einen Nebennieren-Tumor schliessen. Schlussfolgerung: Auch bei klassischen «Schwangerschaftserkrankungen» kann eine internistisch-endokrinologische Ursache zu Grunde liegen. 


\section{P VIII/83 Geburtshilfe: Fallberichte Klinische Erfahrungen mit Bryophyllum pinnatum zur Tokolyse}

Plangger N. ${ }^{\mathrm{a}}$, Rist L. ${ }^{\mathrm{b}}$, Zimmermann R. ${ }^{\mathrm{a}}$, von Mandach U. ${ }^{\mathrm{a}}$

${ }^{a}$ Klinik für Geburtshilfe, Universitätsspital Zürich,

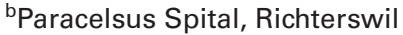

Einführung: Nachdem wir mit dem pflanzlichen Präparat Bryophyllum pinnatum in vitro eine gute Kontraktionshemmung feststellen konnten, wollten wir die klinische Wirksamkeit bei der Tokolyse anhand einer retrospektiven Datensammlung prüfen. Methodik: 67 Schwangere, die mit Bryophyllum pinnatum in einem Alternativmedizinischen Krankenhaus wegen drohender Frühgeburt behandelt wurden (Gruppe 1), wurden 67 Schwangeren mit einer Betamimetikum-Tokolyse aus unserer Klinik (Gruppe 2) gegenübergestellt. Jede Schwangere aus Gruppe 2 musste einer Schwangeren aus Gruppe 1 in folgenden Kriterien entsprechen: mütterliches Alter, Gestationsalter, mittels CTG registrierte Anzahl von Kontraktionen, Muttermundweite, vorzeitiger Blasensprung $\mathrm{ja} /$ nein (alle unmittelbar vor Tokolysebeginn); Frühgeburtenanamnese. Endpunkte der Untersuchung: Schwangerschaftsverlängerung, Gestationsalter bei Geburt, Verträglichkeit bei der Mutter, neonatale Morbidität und Hospitalisationsdauer vor und nach der Entbindung. Resultate: In der B. pinnatum-Gruppe waren die Apgar-Scores bei 1, 5 und 10 min höher, der Sauerstoffverbrauch und die Rate an Atemnotysyndrom tiefer, was in Beziehung mit der niedrigeren, aber nicht signifikanten Frühgeburtenrate gesehen werden muss ( $32.8 \%$ bei B. pinnatum vs. $46.3 \%$, NS). Die mütterliche Verträglichkeit war signifikant besser unter B. pinnatum (weniger Tachykardie und Dyspnoe). Schlussfolgerung: In der Anwendung zur Tokolyse können aufgrund der Ergebnisse keine Nachteile im Sinne einer geringeren Wirksamkeit von B. pinnatum festgestellt werden. Ermutigend ist die gute Verträglichkeit. Die Resultate bilden eine gute Grundlage für eine prospektive kontrollierte Studie zur weiteren klinischen Anwendung von B. pinnatum.

\section{P VIII/84 Geburtshilfe: Fallberichte \\ Evolution d'une grossesse avec une hémoglobinurie paroxystique nocturne}

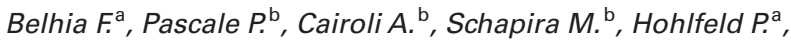
Gerber $S{ }^{a}$

aService de Gynécologie-Obstétrique, Maternité, 'bervice d'Hématologie, Centre Hospitalier Universitaire Vaudois, Lausanne

Introduction: l'hémoglobinurie paroxystique nocturne (HPN) est une maladie rare (1-10/1000000), d'origine acquise. La survie moyenne est de 10-15 ans depuis l'annonce du diagnostique. L'association avec une grossesse est rarement décrite ( $<100$ cas). La mortalité périnatale et maternelle est de 8-20\%. Le tableau clinique se présente par une hémolyse intravasculaire, une neutropénie, une thrombocytopénie et les phénomènes thromboemboliques. L'hémolyse se produit par crises (lors d'infections, douleur et grossesse). La morbidité et mortalité sont surtout liées à ces évènements thrombo-emboliques, artérielles et veineuses. Des rémissions spontanées ont été décrites et le seul traitement curatif est la transplantation de moelle osseuse. Description du cas: Patiente $1 \mathrm{G}$ de 25 ans avec une HPN diagnostiquée en 1999. En cours de grossesse, plusieurs transfusions ont été nécessaires pour maintenir une valeur d' $\mathrm{Hb}>80 \mathrm{~g} / \mathrm{l}$, et une maturation pulmonaire fœtale a été faite à la 28 SA. Aucune anticoagulation prophylactique n'a été admi- nistrée en raison de la thrombopénie $(<20 \mathrm{G} / \mathrm{l})$. La patiente est hospitalisée à $35 \mathrm{SA}$ en raison d'un oligoamnios et une provocation est débutée par misoprostol. Malgré une transfusion plaquettaire, la thrombopénie s'aggravait et des signes de CIVD apparaissaient. Une césarienne médiane sous anesthésie générale a été pratiquée. En cours d'intervention, la patiente reçoit du Novoseven ${ }^{\circledR}$ (facteur de coagulation recombinant VIIa) en raison d'une hémorragie diffuse massive. L'évolution néonatale a été harmonieuse. La phase post-opératoire est marquée par l'apparition d'un syndrome de Budd-Chiari sévère, avec extensions multiples. La patiente est anticoagulée de façon thérapeutique. L'évolution à long terme a été excellente avec reperméabilisation complète de tous les vaisseaux. Elle poursuivra un traitement à vie de Sintrom ${ }^{\circledR}$ et d'ac. folique. Conclusion: Une grossesse dans le cadre d'une HPN est une situation à haut risque, influençant négativement le pronostic clinique de cette maladie, et nécessite une prise en charge multidisciplinaire. Face à une hémorragie sévère, Novoseven ${ }^{\circledR} \mathrm{a}$ eu un effet particulièrement favorable. Mais son indication reste exceptionnelle, au vu des complications majeures possibles.

\section{P VIII/85 Geburtshilfe: Fallberichte \\ Actinomyces neuii als seltene Ursache eines Amnioninfektsyndroms (AIS)}

\author{
Diesch C.H., Raggi A., Di Cienzo G., Holzgreve W.
}

Universitäts-Frauenklinik Basel

Einführung: Wir berichten über eine Frau, bei welcher in der 34. SSW. eine sekundäre Sectio caesarea wegen eines beginnenden AIS durchgeführt wurde. Fallvorstellung: Eine 38-jährige Patientin wurde, durch eine in den USA durchgeführten Oozytenspende, mit dichorialen-diamnioten Gemelli schwanger. Die Eizellspende erfolgte wegen einer primären Sterilität bedingt durch eine seltene autosomal-dominante Ovarialinsuffizienz, welche sich phänotypisch durch ein Blepharophimosis-Ptosis-Epicanthus-inversusSyndrom manifestiert. In der 23. SSW wurde, wegen einer in den Zervikalkanal prolabierenden Fruchtblase eine Notfall-Cerclage durchgeführt. Zweimalige Lungenreifung in der 26. und 33. SSW. In der $33+4$ SSW entwickeln sich zunehmende vorzeitige portiowirksame Kontraktionen. Im Labor zeigen sich leicht erhöhte Entzündungsparameter mit einer Leukozytose von $13700 \times 10^{9} / 1$, einer leichten Linksverschiebung und einem CRP von 20,3 mg/l. Unter dem Gesamtbild einer beginnenden AIS Entscheidung zur sekundären Sectio caesarea. Bei einem Zwilling mit neonatalen Infektzeichen konnten in der Blutkultur Actinomyces neuii nachgewiesen werden. Dieser Erreger konnte im weiteren Verlauf auch im intraoperativ durchgeführten Plazentarabstrich isoliert werden. Weder im Vaginalabstrich noch vom Zahnfleischabstrich, wegen Paradontitis der Patientin, konnten Actinomyces nachgewiesen werden. Diskussion: Das AIS tritt in 0,5-10,5\% der Schwangerschaften auf. Es steht in Assoziation mit 1/3 der Frühgeburten ohne vorzeitigen Blasensprung. Als Risikofaktoren gelten Nulliparität, invasive Pränataldiagnostik, Frühgeburt, mekoniumhaltiges Fruchtwasser, Einlage einer fetalen Pulsoxymetriesonde, pathologischer Vaginalabstrich, Anzahl Vaginaluntersuchungen, vorzeitiger Blasensprung und die Geburtsdauer. Die drei häufigsten Erreger des AIS sind das Ureaplasma urealyticum, die gram-negativen anaeroben Bakterien und das Mycoplasma hominis. Streptokokken der Gruppe B sind für zirka 15\% der AIS verantwortlich. Actinomyces kommen als Verursacher eines AIS äusserst selten vor. Schlussfolgerung: Dieser Fall zeigt, dass man auch an seltene Erreger des AIS denken soll. Actinomyces lassen sich im gewöhnlichen Vaginalabstrich nicht nachweisen. Eine routinemässige Kultur auf Actinomyces scheint unter diesem Aspekt nicht sinnvoll zu sein. 


\section{P VIII/86 Geburtshilfe: Fallberichte Uteriner Gasbrand mit Clostridium perfringens Sepsis nach intrapartaler gedeckter Uterusruptur}

Montavon C., Krause E., Ladewig A., Hösli I., Holzgreve W.

Universitäts-Frauenklinik Basel

Einführung: In der Geburtshilfe sind anaerobe Infektionen mit Clostridium perfringens (CP) eine Rarität, allerdings mit einer hohen maternalen Mortalität von $50-80 \%$. Wir berichten über eine Patientin, bei der sich postpartal ein uterines Gas-Gangrän entwickelt hat, und diskutieren das Management. Wir hoffen damit die Aufmerksamkeit für diese Infektion zu wecken. Kasuistik: Bei der 28-jährigen GII, PII mit Status nach Sectio in 2002 wurde 48 h nach Vakuumextraktion wegen akutem Abdomen eine Laparotomie bei Verdacht auf gedeckte Uterusruptur durchgeführt. Intraoperativ zeigte sich bei intakter Uterotomienarbe ein parametraner Riss, der übernäht wurde. Die antibiotische Abschirmung erfolgte mit Tazobactam. $36 \mathrm{~h}$ später verschlechterte sich der Allgemeinzustand schlagartig. Der Verdacht einer Cellulitis wurde früh geäussert und nach Erhalt der Abstrichresultate (CP) aus Uterus und Hämatom zusätzlich mit Clindamycin behandelt. Trotzdem konnte ein sich rasant entwickelndes Gas-Gangrän beobachtet werden, mit einseitiger Ausbreitung über das Abdomen bis in die linke Flanke zur Axilla und zum Oberschenkel. Die Patientin äusserte zunehmende Schmerzen, zuerst mit wenig klinischem Korrelat, dann bronzeartiger Hautverfärbung, später Krepitationen und Todesangst. Entscheidend für die erfolgreiche Behandlung, die das Überleben der Patientin gesichert hat, war trotz hoch dosierter resistenzgerechter Antibiose ein aggressives Management mit Hysterektomie, Nekroseausräumung und Faszienspaltung, gefolgt von mehrmaligem Débridement und offener Behandlung, um die anaeroben Bedingungen zu unterbrechen. Die Patientin konnte nach 21 Tagen entlassen werden. Sie entwickelte jedoch ein posttraumatisches Syndrom mit schweren depressiven Episoden und wird psychologisch betreut. Schlussfolgerung: Clostridium perfringens ist ubiquitär, in 1-10\% auch vaginal bei gesunden Frauen zu finden. Sein Nachweis hat noch keine pathologische Bedeutung. Die Infektion aber, die sich in geeignetem Milieu entwickelt, von Endometritis bis zur Sepsis reichend, kann fulminant laufen. Eine Früherkennung und ein bewusstes interdisziplinäres Management sind von zentraler Bedeutung. Die aggressive chirurgische Behandlung mit Hysterektomie ist, kombiniert mit der entsprechenden Antibiotikatherapie, lebensrettend.

\section{P VIII/87 Geburtshilfe: Fallberichte Fallvorstellung: Schwangerschaft unter Therapie mit Aromatase-Inhibitoren bei schwerer infiltrierender Endometriose}

Rossi Montalbetti F. ${ }^{\mathrm{a}}$, Venzi G. ${ }^{\mathrm{b}}$, Safwan S. ${ }^{\mathrm{a}}$, Tosi C. ${ }^{\mathrm{c}}$, Gyr Th. $^{\mathrm{a}}$

${ }^{a}$ Klinik für Gynäkologie und Geburtshilfe, ${ }^{\text {b} K l i n i k ~ f u ̈ r ~ U r o l o g i e, ~}$ ${ }^{\mathrm{c}}$ Klinik für Neurologie, Ospedale Civico, Lugano

Einleitung: Die Endometriose kann chronische Schmerzen, Sterilität und Organschäden verursachen. Das Therapiekonzept stellt den Gynäkologen oft vor Probleme, da die Behandlung der einzelnen Teilsymptome unterschiedlich ist. Fallbericht: Eine 29jährige Nulligravida wurde im Mai 2002 wegen Hydronephrose links hospitalisiert. Die gynäkologische Untersuchung ergab den Verdacht auf eine ausgedehnte tiefe infiltrierende Endometriose des Ligamentum cardinale bis an die Beckenwand links mit Infiltration bzw. Perforation der Vagina bis $1 \mathrm{~cm}$ an den Hymenalsaum. Es wurde deshalb eine Exzision der Endometriose in Laparoskopie und eine Resektion mit Reimplantation des Ureters durchgeführt. Bei Ausdehnung in den Obturatoriuskanal und bis and die Beckenwand musste auf eine radikale Ausräumung verzichtet werden. Nach einer symptomfreien Periode unter Ovulationshemmer im März 2003 erneute Manifestation der Endometriose in Form von neurologischen Ausfällen im Sinne von Parästhesien im linken Bein. Im CT Nachweis von Endometrioseherden extraspinal auf Höhe von L4, L5 und S1. Unter GnRH-Analoga (Zoladex) in Kombination mit Anastrozol $1 \mathrm{mg} / \mathrm{d}$ (Arimidex) Besserung der Symptomatik. Im Sommer 2003 Versuch einer FIVET. Unter ovarieller Stimulation schlagartige Wiederauftreten der neurologischen Sympomatik sowie von Schmerzen. Deshalb erneut Therapie mit GnRH-Analoga und Anastrozol. Nach Abklingen der Symptomatik Absetzen der GnRH-Analoga und Weiterführen der Therapie mit Arimidex. Unter Anastrozol $1 \mathrm{mg}$ pro Tag Auftreten einer spontanen Schwangerschaft. Die Medikation wurde abgesetzt und nach unauffälligem Schwangerschaftsverlauf sekundäre Sectio am Termin eines gesunden Kindes. Schlussfolgerung: Die schwere Endometriose stellt hohe Anforderungen an den behandelnden Gynäkologen. Die Operation ist die Therapie der Wahl, kann im Einzelfall jedoch unter Umständen nicht radikal erfolgen. Medikamentöse Behandlungen sind vor allem bei Kinderwunsch unbefriedigend. Anastrozol ist in Spezialfällen eine mögliche Alternative.

\section{P VIII/88 Geburtshilfe: Fallberichte Choc anaphylactique et grossesse}

Etessamipour $B$.

Service de Gynécologie, Hôpital de La Chaux-de-Fonds

Introduction: Le choc anaphylactique est une hypovolémie relative due à vasoplégie causée par libération des médiateurs vasoactifs. Un choc durant la grossesse met le fœtus en danger d'hypoxie importante avec des répercussions respiratoires et surtout neurologiques irréversibles. Le cas clinique: Patiente de 29 ans, primigeste, à 37 semaines de grossesse, en bon état général, sans allergie connue. Après une grillade, elle ressent des douleurs lombaires, fourmillements diffus, dyspnée et perte de connaissance. Al'arrivée des ambulanciers une première tension artérielle après le remplissage est de 50/20 mm Hg. Elle présente par la suite des lésions érythémateuses diffuses. C'est avec l'adrénaline que la tension remonte à 120/70 mm Hg. Elle reçoit solumédrol et tavegyl intraveineux, avec la disparition des lésions cutanées rapidement. La patiente se porte bien. Arrivée en salle d'accouchement, au CTG on a une souffrance fœtale aiguë, une césarienne en urgence sous anesthésie générale est effectuée. Un liquide amniotique clair puis sanglant avec la naissance d'un garçon pesant $3050 \mathrm{~g}$ et un $\mathrm{pH}$ du cordon 7,15 et 7,16, Apgar 3/6/7. Un syndrome de détresse respiratoire d'emblée avec fasciculations de langue et pupilles en myosis serré, évoquant une atteinte neurologique compatible avec une asphyxie prénatale. Un EEG montre un tracé aréactif (mort cérébrale) donc la décision d'arrêt des soins a été prise. Discussion: Des lésions cutanées et une évolution en quelques minutes vers un choc qui a été rapidement réversible après remplissage et adrénaline et une élévation de tryptase diagnostiqué par la suite, sont évocateurs un choc anaphylactique de stade IV selon Müller. Le diagnostic différentiel probable était l'embolie du liquide amniotique (nommée par certains auteurs le syndrome anaphylactique de la grossesse) causé surtout par des particules véhiculées dans les capillaires pulmonaires provocants le spasme réactionnel et une 
réaction anaphylactique. En l'occurrence, les investigations allergologique ont confirmés une réaction anaphylactique sur hypersensibilité à la tomate. Conclusion: Lors d'un choc durant la grossesse, l'objectif principal est le traitement étiologique du choc en stabilisant les conditions hémodynamiques de la patiente (tension artérielle dans notre cas) et l'extraction fœtale, ainsi on peut sauvegarder la vie de la patiente et du fœtus.

\section{P IX/90 Gynäkologie \\ Akuter Unterbauchschmerz und vaginale \\ Schmierblutung als Manifestation eines \\ Hämangioms des Corpus uteri - \\ Ein Fallbeispiel \\ Scherer M. ${ }^{a}$, Hung B. ${ }^{a}$, Yeginsoy $S .^{b}$, Burger D.A. ${ }^{a}$

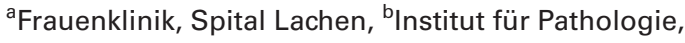 StadtspitalTriemli, Zürich}

Einführung: Eine abnorme vaginale Blutung bei perimenopausalen Frauen wird oftmals durch benigne, seltener durch maligne Neubildungen des Corpus uteri oder der Cervix verursacht. Häufig findet man Corpuspolypen als meist benigne glandulär zystisch veränderte Hyperplasien des Endometriums. Hämangiome im Uterus sind dagegen selten. Fallbeschreibung: Notfallmässige Zuweisung einer 52-jährigen Patientin wegen akut eingetretener, massiver Unterbauchschmerzen. Bei Eintritt vaginale Schmierblutung seit 14 Tagen bei St.n. letzter Menstruation vor 6 Monaten. Sonographisch lässt sich in der Cervix ein suspekter zystischer Befund von etwa $4 \mathrm{~cm}$ Durchmesser darstellen. Bimanuell ausgeprägter Portioschiebe- und -hebeschmerz. Bei der Spekulumeinstellung entleert sich nach Manipulation mit einem Watteträger am äusseren Muttermund viel Schleim und Altblut, worauf die Patientin schlagartig praktisch beschwerdefrei wird. Bei der diagnostischen Hysteroskopie mit anschliessender fraktionierter Curettage kommt eine polypöse Struktur im Cervikalkanal zu Darstellung, die ihren Ursprung an der Hinterwand des Fundus uteri hat. Die Histologie ergibt einen stark entzündlich und fokal nekrotisch-phlegmonös durchsetzten vaskulären Polypen, am ehesten einem Hämangiom entsprechend. Der postoperative Verlauf gestaltet sich komplikationslos. Die Patientin kann in gutem Allgemeinzustand nach Hause entlassen werden. Schlussfolgerung: Hämangiome im Uterus sind meist Zufallsbefunde. Durch Hämangiome hervorgerufene vaginale Blutungen sind bisher in der Literatur nur wenig beschrieben worden. Als Ursache der Schmerzen fand sich bei der oben beschriebenen Patientin ein Hämangiom in statu nascendi, eine seltene Differentialdiagnose bei Unterbauchschmerzen.

\section{P IX/91 Gynäkologie \\ Eitrige Adnexitis mit Perihepatitis: Eine Präsentation des familiären Mittelmeerfiebers \\ Kaplan Z. ${ }^{\mathrm{a}}$, Bass B. ${ }^{\mathrm{a}}$, Von Castelberg B. ${ }^{\mathrm{a}}$, Bühlmann U. ${ }^{\mathrm{b}}$, Korte $\mathrm{W}^{\mathrm{c}}$ \\ ${ }^{a}$ Frauenklinik Maternité, StadtspitalTriemli, Zürich, \\ ${ }^{b}$ Klinik für Kinder und Jugendliche, Stadtspital Triemli, Zürich, \\ ${ }^{\mathrm{C}}$ Institut für Klinische Chemie und Hämatologie, Kantonsspital St. Gallen}

Einführung: Das familiäre Mittelmeerfieber (FMF) ist eine genetische Erkrankung infolge einer Mutation des MEFV-Gens auf dem Chromosom 16. Die typischen Symptome sind rezidivierende
Tabelle 1. Tel-Hashomer-Kriterien zur Diagnose eines FMF

\begin{tabular}{ll}
\hline Majorkriterien & Minorkriterien \\
\hline $\begin{array}{l}\text { Rezidivierendes Fieber mit Peritonitiden, } \\
\text { Synovitiden oder Pleuritiden }\end{array}$ & Rezidivierendes Fieber \\
Amyloidose vom AA-Typ & Erysipelähnliche Erytheme \\
Ansprechen auf Kolchizin & FMF beim erstgradigen \\
& Verwandten \\
\hline
\end{tabular}

Fieberschübe, Serositiden (Peritonitis, Arthritis, Pleuritis) und erysipeloidartige Erytheme. Die häufigste Todesursache ist die terminale Niereninsuffizienz als Folge einer Amyloidose. Kasuistik: Ein 13-jähriges Mädchens wird wegen Unterbauchschmerzen, Fieber $\left(38,7^{\circ} \mathrm{C}\right)$, erhöhtem CRP $(158 \mathrm{mg} / \mathrm{l})$ und Leukozytose $\left(13.8 \times 10^{9} /\right.$ 1) hospitalisiert. Ähnliche Episoden von Bauchschmerzen und Fieber sind in den letzten Jahren wiederholt aufgetreten. Die Laparoskopie, die bei Verdacht auf Appendizitis acuta durchgeführt wird, zeigt eine eitrige Adnexitis rechts mit Perihepatitis. Die antibiotische Therapie mit Augmentin und Vibramycin führt zur raschen Besserung. Wiederholte Abklärungen auf Chlamydien blieben negativ. Erneute Episoden mit Unterbauchschmerzen, Fieber, CRPAnstieg und Leukozytose sprechen auf Paracetamol an. In der Familienanamnese der sephardischen Juden konnte nach einiger Zeit der Grossvater und eine Grosstante väterlicherseits mit ähnlichen Beschwerden eruiert werden. Die genetische Analyse zeigt eine M694V und V726A Mutationen auf dem Chromosom 16. Unter Colchizin $(1 \mathrm{mg} / \mathrm{d})$ bleibt die Patientin im weiteren Verlauf beschwerdefrei. Diskussion: Bei rezidivierenden Peritonitiden ohne offensichtliche Ursache ist ein FMF zu erwägen. Die Verdachtsdiagnose wird aufgrund der Klinik gestellt (Tabelle 1) und durch Nachweis der genetischen Mutation gesichert. Schlussfolgerung: Bei Mädchen und Frauen mit Fieberepisoden und rezidivierenden Bauch- und insbesondere Unterbauchschmerzen ohne klare Ätiologie ist ein FMF in Betracht zu ziehen.

\section{P IX/92 Gynäkologie \\ PAPP-A und Osteoprotegerin, zusammen mit Interleukin-8 und RANTES, sind erhöht in der Peritonealflüssigkeit von Frauen mit Endometriose}

Bersinger N.A., Von Roten S., Wunder D.M., Raio L., Dreher E., Müller M.D.

Universitäts-Frauenklinik, Bern

Enführung: Interleukin-8 (IL-8) und Regulated on Activation, Normal T cell Expressed and Secreted (RANTES) zeigen bei Frauen mit Endometriose erhöhte Werte in der Peritonealflüssigkeit. Dies wurde neulich auch für die Proteine Pregnancy-associated Plasma Protein A (PAPP-A), Glycodelin (PP14), Osteoprotegerin (OPG), und lösliches CD163 (sCD163) vorgeschlagen. In dieser Studie ging es darum, aufzuzeigen welche dieser «neuen» Marker tatsächlich erhöht sind, und in diesem Falle das Ausmass dieses Effektes untereinander und mit IL-8 und RANTES zu vergleichen. Material und Methoden: Immuno-analytische Quantifizierung von RANTES, PAPP-A, Glycodelin, sCD163, OPG, und als Backup Progesteron im Douglaspunctat von 132 Frauen, die sich zur Evaluation von Infertilität, Schmerzen im Pelvis, oder zur tubalen Sterilisation einer Laparoskopie unterzogen. Von diesen zeigten 55 keine Endometriose, 18 wurden als rAFS I oder II, und 59 als rAFS 
III oder IV eingestuft. Resultate: Neben den Chemokinen IL-8 und RANTES zeigten sich auch die medianen Konzentrationen von PAPP-A und OPG in der Peritonealflüssigkeit bei Frauen mit Endometriosie signifikant erhöht, und dies ebenfalls in Abhängigkeit des Schweregrades der Pathologie. Während Glycodelin zwar auch erhöht war, aber eine starke Streuung zeigte, fand sich sCD163 unverändert. Die meisten untersuchten Marker zeigten signifikante, positive Korrelationen untereinander (Spearman Rank Correlation Analysis). Schlussfolgerung: PAPP-A und OPG dürften wie IL-8 und RANTES eine Rolle im Inflammationsprozess bei der Endometriose spielen. Das PAPP-A hat den Vorteil, dass es in der Klinik bereits routinemässig angewendet wird (Pränataldiagnostik); in dieser Studie zur Endometriose waren aber IL-8 und RANTES dem PAPP-A und OPG statistisch leicht überlegen.

\section{P IX/93 Gynäkologie \\ Pelviner Morbus Castleman: Fallbericht einer seltenen gynäkologischen Diagnose}

Venturelli S. ${ }^{a}$, von Orelli S. ${ }^{a}$, Montani M. ${ }^{c}$, Lauper U. ${ }^{a}$, Salomon $F^{\mathrm{b}}$, Fink $D$. $^{\mathrm{a}}$

aKlinik für Gynäkologie und Geburtshilfe, Departement

Frauenheilkunde, 'bepartement Innere Medizin,

'Departement Pathologie, Universitätsspital Zürich

Einführung: Der Morbus Castleman ist eine seltene benigne Erkrankung unbekannter Ätiologie, die durch Proliferation des lymphatischen Gewebes charakterisiert ist. Histopathologisch werden zwei Typen unterschieden, ein hyalin-vaskulärer und ein Plasmazelltyp. Beide Arten können sich als lokalisierte oder disseminierte Form präsentieren. Bei 70\% der Patienten befindet sich dieser Tumor im Mediastinum. In der gynäkologischen Praxis ist diese Krankheit extrem selten. Wir berichten über eine Patientin mit einem M. Castleman in der Fossa obturatoria rechts. Fallbericht: Eine 53-jährige II Gravida, I Para leidet an anämisierenden Hypermenorrhoe und Unterbauchschmerzen rechts. In der transvaginalen Sonographie wird ein hochaufgebautes Endometrium und eine vergrösserte rechte Adnexe festgestellt. Eine Hysteroskopie mit Curettage und Therma choice-Endometriumablation wird durchgeführt. 6 Wochen postoperativ meldet sich die Patientin bei uns für eine zweite Meinung, da sie wieder an Hypermenorrhoe und zunehmenden Schmerzen im Unterbauch rechts leidet. Die transvaginale Ultraschallkontrolle zeigt in der rechten Adnexloge parauterin einen rundlichen echoreichen $5 \mathrm{~cm}$ messenden Befund mit zentraler Verflüssigung. Eine laparoskopische Hysterektomie mit Adnexektomie rechts wird geplant. Intraoperativ präsentiert sich ein plumper Uterus mit unauffälligen Adnexen beidseits. In der Fossa obturatoria rechts zeigt sich aber eine $4 \times 3 \mathrm{~cm}$ grosse Raumforderung, die nach der laparoskopischen Hysterektomie entfernt wird. Die Histologie ergibt Lymphknotengewebe mit Morbus Castleman vom hyalinen vaskulären Typ. In einer postoperativen CT-Kontrolle sind noch zwei $2,5 \mathrm{~cm}$ grosse Lymphknoten iliacal rechts zu sehen, welche die radiologischen Kriterien des M. Castleman erfüllen. Die Patientin wird keine weiteren Behandlungen benötigen, da es sich um eine lokalisierte gutartige Form der Krankheit handelt und die Schmerzen postoperativ regredient sind. Es werden nur klinische und radiologische Kontrollen alle 6 Monate stattfinden. Schlussfolgerungen: Bei unklarem echoreichen sonographischen Adnexbefund kann in seltenen Fällen ein M. Castleman vorliegen.

\section{P IX/94 Gynäkologie Aussergewöhnliche Differentialdiagnose eines zystischen Unterbauchtumors}

\author{
Scherrer R., Agorastos S., Dombi V., Honegger Ch.
}

Frauenklinik, Spital Uster

Einführung: Die weitgefächerte Differentialdiagnose von Unterbauchschmerzen bei der Frau stellt uns immer wieder einmal vor diagnostische Schwierigkeiten. Hier berichten wir über einen aussergewöhnlichen Fall eines schmerzhaften Unterbauchtumors bei einer jungen Frau. Material und Methode: Fallbericht über ein 14-jähriges Mädchen, Virgo intacta mit progredienter Dysmenorrhoe, regelrechter Menarche mit 12 Jahren, bekannter kongenitaler Nierenagenesie rechts. Notfallmässige Zuweisung wegen rezidivierenden Episoden von akuten UB-Schmerzen bei abdominal-sonographisch nachgewiesenem zystischem Befund im rechten Unterbauch von $8 \times 7 \times 7 \mathrm{~cm}$ Grösse. Ergebnisse: Bei V.a. riesige Adnexzyste DD Endometriom, Teratom wurde die Indikation zur diagnostisch-interventionellen Laparoskopie gestellt. Intraoperativ zeigten sich unauffällige Adnexen bds, jedoch ein Uterus bicornis DD Uterus didelphys mit unklarer aufgetriebener Struktur im Bereich des isthmo-cervikalen Überganges. Frustraner Punktionsversuch desselben. Das postoperative MRI bestätigte die Verdachtsdiagnose eines Uterus didelphys und zeigte einen Hemihämatokolpos unilateralis rechts bei Doppelanlage der Vagina. Dabei staute sich das Menstrualblut in der rechten nicht durchgängigen Hemivagina, was sonographisch als zystischer Unterbauchtumor imponierte. In einer zweiten Operation erfolgte dann die Inzision und partielle Resektion des Vaginalseptums am caudalen Pol zur Drainage des angestauten Menstrualblutes. Bei Aufnahme des Geschlechtverkehrs wird eine vollständige Resektion des Vaginalseptums zu diskutieren sein. Schlussfolgerungen: Bei adoleszenten Patientinnen sollte bei Symptomen wie progrediente Dysmenorrhoe und UB-Schmerzen bei regelhafter Menarche und regelmässiger Menstruation differentialdiagnostisch an genitale Fehlbildungen gedacht werden.

\section{P IX/95 Gynäkologie Angiomyofibroblastome de la vulve}

Temgoua A., Kudla A., Burger H., Sapin B.

Hôpital intercantonal de la Broye, Payerne

Introduction: Nous décrivons un cas rare d'angiomyofibroblastome de la vulve, tumeur mésenchymale myxoide, bénigne, rare. Présentation du cas: Patiente de 41 ans, sous contraception orale, 4G1P chez qui on découvre lors de la consultation un nodule de 3 $\mathrm{cm}$ au niveau de la grande lèvre gauche, mou, indolore, mobile, sans réaction inflammatoire périphérique. Le reste du status gynécologique est dans la norme. L'US montre une image homogène de $3 \times 1,5 \mathrm{~cm}$. Biopsie - excision de ce nodule, se trouvant encapsulé avec une extirpation aisée, qui s'avère être un angiomyofibroblastome (AMF). Description macroscopique: Fragment polypoide mesurant $3 \times 2 \times 1,5 \mathrm{~cm}$ avec une base d'implantation de $0,2 \mathrm{~cm}$. La surface externe est lisse. A la coupe, aspect homogène de coloration beige-brunâtre. Périphériquement, cette tumeur présente une capsule en partie collagénique et régulièrement apparente. La patiente a été revue 1 année plutard sans complications ni récidives locales. Discussion: L'angiomyofibroblastome est une tumeur mésenchymale myxoide, bénin, extrêmement rare, qu'on trouve en générale dans la région vulvaire chez la femme avant la ménopause. Pathologie décrite pour la 1ère fois par Fletcher 
comme tumeur bénigne vulvovaginal, sous-cutanée et bien circonscrite. Toute fois on note d'autres localisations de cette lésion au niveau du tractus génital: Trompes de Fallope, portion vaginale du col, vagin, région inguinale, périnée, et scrotum chez l'homme. Jusqu'en 2003, sur les 76 cas reportés chez la femme, 79\% des AMF étaient localisées à la vulve. Il est important de différencier histomorphologiquement l'AMF de l'angiomyxome aggressif qui n'est autre qu'une tumeur mésenchymale myxoedémateuse au stroma unique associé à un risque élevé de récidive locale mais sans potentiel métastatique, ainsi que des autres tumeurs de la vulve. Conclusion: Dans l'AMF de la vulve, les patientes se présentent avec une masse vulvaire, indolore, posant le diagnostique différentiel d'une pathologie de la glande de Bartholin ou d'autres tumeurs (lipome). Le seul traitement consiste en une excision chirurgicale en tissus sain, afin d'éviter tout risque de récidive et de permettre par l'analyse pathologique d'exclure tout autre néoplasie de la vulve.

\section{P IX/96 Gynäkologie \\ Das hereditäre Angioödem: Verlauf in der Schwangerschaft, unter oralen Kontrazeptiva und HRT}

Reinke C. ${ }^{\mathrm{a}}$, Kuhn A. ${ }^{\mathrm{b}}$, Bork K. ${ }^{\mathrm{a}}$

aHautklinik, Johannes Gutenberg Universität, Mainz, Deutschland, ' Frauenklinik Bern

Einleitung: Das hereditäre Angioödem (HAE) tritt mit einer Inzidenz von 1:10 000 bis 1:50 000 auf und wird je nach $C 1$ Inhibitor Funktionsstörung in die Klassen I-III eingeteilt. Ziel dieser Studie ist die Beantwortung der Frage, ob es tatsächlich einen $\mathrm{Zu}-$ sammenhang mit den HAE-Attacken und Schwangerschaft sowie exogenen Hormonen gibt. Patientinnen und Methode: Mittels Krankengeschichten wurde alle Patientinnen erfasst, die in der Hautklinik der Universität Mainz zwischen 1980 und 2004 wegen HAE-behandelt wurden. Diese Patientinnen wurden mittels Fragebogen und für ergänzende Fragen telefonisch kontaktiert und hinsichtlich Schwangerschaftsverlauf, Verlauf des HAE unter oralen Kontrazeptiva und hormoneller Ersatztherapie, gynäkologischen Nebendiagnosen und Nachkommenschaft mit HAE befragt. Resultate: Es wurden 60 Patientinnen mit HAE identifiziert, wovon 40 unter Typ I, vier unter Typ II und 16 unter Typ III litten. 42 Patientinnen hatten Schwangerschaften erlebt, wobei der Verlauf der HAE $13 \mathrm{mal}$ in der Schwangerschaft besser wurde, $5 \mathrm{mal}$ gleich blieb und sich 23 mal verschlechterte unabhängig vom Gestationsalter. Es gab eine Erstmanifestation in der Schwangerschaft. Intrapartal hatte eine Patientin eine Attacke und bei $7 \mathrm{~Pa}-$ tientinnen kam es $48 \mathrm{~h}$ postpartal zu einem Anfall. 13 Patientinnen erlebten einen oder mehrere Aborte. Von 53 Pillenbenutzerinnen kam es in 9 Fällen zu HAE ausschliesslich unter oralen Kontrazeptiva, und 29 Frauen beendeten die Pilleneinnahme oder wechselten wegen HAE das Präparat. 44 Patientinnen erlebten eine Verschlechterung unter oralen Kontrazeptiva. Von 9 Frauen unter HRT gab es 5 Verschlechterungen unter Hormoneinnahme, 4 Patientinnen ohne Veränderung und keine Erstmanifestation. 20 Nachkommen aus 19 Familien haben HAE in der nächsten Generation gezeigt. Diskussion: HAE kann ganz offensichtlichdurch Schwangerschaft und exogene Hormongaben negativ beeinflusst werden. Die Inzidenz der Aborte erscheint erhöht, sollte aber noch altersadaptiert betrachtet werden. Patientinnen mit HAE sollten in einer Schwangerschaft darüber informiert werden, dass eine Verschlechterung des HAE wahrscheinlich ist. Es sollten möglichst Alternativen zur oralen hormonellen Kontrazeption diskutiert werden.

\section{P IX/97 Gynäkologie \\ Etude descriptive des urgences chirurgicales gynécologiques à I'Hôpital Cantonal de Genève entre 2000 et 2004}

Samba-Nganga A.B., Daniel F., Sandrine J., Dubé M.J., Dubuisson J.B.

Département de Gynécologie-Obstétrique,

Hôpitaux Universitaires de Genève

Introduction: Nous avons étudié l'évolution des urgences chirurgicales gynécologiques, durant les 5 dernières années. Matériel et méthode: A partir de la base de donnée des activités opératoires du service, nous avons répertorié les urgences chirurgicales gynécologiques et analysé les proportions des différents types de chirurgies réalisées ces 5 dernières années. Résultats: Durant ces 5 dernières années on a noté une augmentation importante des interventions électives contrairement aux interventions en urgence. On note une diminution légère d'urgences chirurgicales le week-end ( $\mathrm{p}=$ $0,001)$. Les urgences chirurgicales les plus fréquentes sont: les curetages évacuateurs (56\%), les laparoscopies (27\%), les bartholinites (7\%). Durant ces 5 dernières années le nombre de curetages évacuateurs a augmenté, le nombre de laparoscopies a diminué et le nombre de bartholinites est resté stable (tableau 1). Conclusion: L'augmentation du nombre d'interventions chirurgicales entre l'année 2000 et 2004 n'est pas associée à une augmentation du nombre des interventions faites en urgence. Le nombre élevé de laparoscopies en urgence nécessiterait la présence permanente de médecins habilités à le faire.

\section{Tableau 1}

\begin{tabular}{lrrrrr}
\hline Année & 2000 & 2001 & 2002 & 2003 & 2004 \\
\hline Elective & 802 & 880 & 886 & 1,028 & 1,479 \\
Urgente & 379 & 434 & 405 & 412 & 383 \\
Urgence pendant le week-end & 101 & 106 & 86 & 99 & 72 \\
Curetage & 173 & 234 & 231 & 261 & 225 \\
Laparoscopies & 131 & 125 & 95 & 87 & 102 \\
Bartholinites & 31 & 27 & 27 & 25 & 26 \\
\hline
\end{tabular}

\section{P IX/98 Gynäkologie Kystes ovariens et pertinence échographique}

Damnon F., Beurret Lepori N.

Département de Gynécologie-Obstétrique, Centre Hospitalier Universitaire Vaudois, Lausanne

Introduction: Corréler de façon rétrospective les données échographiques des kystes ovariens avec les résultats d'anatomopathologie, et analyser la performance de l'échographiste expérimenté ou non. Matériel et méthodes: Durant l'année 2004, tous les cas de kystes ovariens diagnostiqués échographiquement dans notre département ont été revus. Les kystes ont été classés comme: kystes fonctionnels, kystes du corps jaune, endométriomes, kystes dermoïdes, kystes organiques liquides, kystes organiques solides, kystes organiques solido-liquides et cancers ovariens selon les critères de Sassone, sans analyse Doppler pour la majorité des cas. Les échographistes ont été divisés en deux groupes: avec ou sans formation échographique spécifique. Les résultats anatomopathologiques pour les patientes opérées ont été revus. Résultats: 467 diagnostics 
de kystes ovariens ont été posés durant cette période, 114 ont été traités chirurgicalement: 203 kystes fonctionnels (33 opérés dont 7 avec diagnostic non-concordant DNC), 67 kystes du corps jaune ( 9 opérés dont 2 avec DNC), 33 endométriomes (10 opérés dont 2 avec DNC), 21 kystes dermoïdes (14 opérés dont 3 avec DNC), 98 kystes organiques liquides (22 opérés dont 1 avec DNC), 6 kystes organiques solides ( 3 opérés dont 2 avec DNC), 32 kystes organiques solido-liquides ( 20 opérés dont 4 avec DNC) et 7 cancers ovariens ( 3 opérés dont $1 \mathrm{DNC}$ ). La concordance échographique est présente pour $78 \%$ des cas. Deux cas de cancers n'ont pas été diagnostiqués correctement, un d'origine ovarienne et l'autre d'origine digestive. Plus l'échographiste est expérimenté, plus la concordance échographique est présente, elle n'est cependant pas statistiquement significative. Conclusions: Des critères échographiques simples ainsi qu'une formation échographique sont indispensables afin de classer les kystes ovariens et ainsi de définir une attitude. Une concordance entre l'échographie et les résultats chirurgicaux est retrouvée pour $78 \%$ des cas.

\section{P X/100 Gynäkologie \\ GnRH-Analoga: Therapieoption bei aggressivem Angiomyxom}

Markus A., Eberhard M., Litschgi M.

Abteilung für Gynäkologie und Geburtshilfe,

Kantonsspital Schaffhausen

Einführung: Das aggressive Angiomyxom ist ein seltener Weichteiltumor, welcher sich vorwiegend in der Becken- und Genitalregion manifestiert. Er imponiert durch langsames und destruktives Wachstum ohne Metastasierung und hat eine hohe Rezidivrate. Erstmals beschrieben wurde der Tumor 1983. 1993 wurde entdeckt, dass alle aggressiven Angiomyxome einen positiven Östrogen- und Progesteronrezeptor haben. In dem hier vorliegenden Fallbericht wird ein Ansprechen eines aggressiven Angiomyxoms auf GnRH-Analoga beschrieben. Material und Methoden: Im September 1996 stellte sich die Patientin erstmals mit dem Verdacht auf eine chronische rezidivierende Bartholinische Zyste vor und es erfolgte die Tumorektomie. Histologisch zeigte sich ein aggressives Angiomyxom der Vulva, welches im Gesunden exzidiert wurde. Die bestimmten Hormonrezeptoren für Östrogen und Progesteron waren positiv. Nachfolgend wurden mehrere Nachoperationen wegen eines Rezidivs, sowie Rekonstruktionen des Beckenbodens, durchgeführt. Im November 2001 trat erneut ein Tumorrezidiv auf. Da die Patientin weitere Operationen ablehnte, begannen wir im November 2002 über einen Zeitraum von 10 Monaten mit der monatlichen intramuskulären Injektion von 4,12 mg Triptorelinacetat (Decapeptyl retard). Um die unter der Therapie auftretenden postmenopausalen Beschwerden zu lindern, erfolgte die gleichzeitige Add-back-Therapie mit $2 \mathrm{mg}$ Estradiol (Estrofem). Nach 4-monatiger und 10-monatiger Decapeptyltherapie wurden bei der Patientin multiple Probenentnahmen aus den vormals tumorpositiven Geweben entnommen. Ergebnisse: Alle entnommenen Gewebeproben waren sowohl nach 4-monatiger als auch nach 10-monatiger Therapie mit GnRH-Analoga tumorfrei. Schlussfolgerung: Die primäre Therapie sollte in einer möglichst vollständigen chirurgischen Sanierung bestehen, um ein Rezidiv zu vermeiden. Bei positivem Östrogen- und Progesteronrezeptorstatus sollte die Therapie mit GnRH-Analoga durchgeführt werden. Zur Verlaufskontrolle eignen sich MRI-Untersuchungen.

\section{P X/101 Gynäkologie \\ Kombinierte Behandlung von vaginalen und vulvären Dysplasien mittels $\mathrm{CO}_{2}$-Laser und Imiquimod}

\author{
Lengsfeld D., Dreher E., Müller M.D.
}

Frauenklinik, Inselspital Bern

Einleitung: Dysplastische Veränderungen der Vulva und Vagina (VIN/VAIN) sind seltene Erkrankungen, wobei die vulvären Veränderungen (Inzidenz: 1,6:100 000) tendenziell zunehmen und häufiger symptomatisch sind als die vaginalen (VAIN: 1:100 000). Als Risikofaktoren für beide Erkrankungen gelten St.n. Cervixneound dysplasien, assoziierte HPV-Infektionen, Nikotinabusus und Immunsuppression. Die Rezidivrate nach verschiedenen Therapien ist hoch und die Belastung dieser Patienten enorm. Unser Ziel war es, den Effekt der Kombination von Operation und Imiquimod auf die Rezidivhäufigkeit zu untersuchen. Patienten und Methode: Zwischen 1/2002 und 1/2005 wurden im Rahmen unserer Dysplasiesprechstunde 23 Patienten mit VIN (19) und VAIN (4) behandelt. In $17(13 \times$ VIN, $4 \times$ VAIN) der 23 Fälle kam prä- oder postoperativ Imiquimod zum Einsatz. Die Frauen wurden instruiert, Imiquimod $3 \times$ wöchentlich für $6 \mathrm{~h}$ anzuwenden. Bei massiven Nebenwirkungen sollten sie entweder das Intervall verlängern oder die Einwirkzeit verkürzen, wobei jedoch eine Wirkung in Form leichter Nebenwirkungen nachweisbar bleiben sollte. Der Behandlungserfolg wurde anhand kolposkopischer, zytologischer bzw. bioptischer Kontrollen bewertet. Resultate: Für 3 von 4 Patienten mit VAIN II-III, die prä- oder postoperativ mit Aldara behandelt wurden, konnte eine Heilung nachgewiesen werden, in einem Fall war noch ein VAIN I nachweisbar (mittlere Beobachtungszeit: 13 Monate, Range: 7-20 Monate). Bei vulvärer Dysplasie war die zusätzliche Behandlung mittels Imiquimod bis jetzt in 7 Fällen erfolgreich (mittlere Beobachtungszeit: 19 Monate, Range: 5-35), bei 4 Patienten ist sie noch nicht abgeschlossen. In einem Fall war nach kombinierter Behandlung eines VIN III noch ein VIN I nachweisbar, in einem weiteren zeigte der Abstrich bei klinisch rückläufigen Veränderungen einen ASCUS. Das von den Frauen bestimmte Applikationsschema war sehr individuell, die herkömmliche Dosierung für fast alle Frauen zu hoch. Schlussfolgerungen: Die vorläufigen Ergebnisse zeigen die Effektivität der prä- bzw. postoperativen Imiquimodtherapie sowohl bei VIN als auch bei VAIN hinsichtlich einer verminderten Rezidivrate. Für endgültige Aussagen bedarf es jedoch einer längeren Beobachtungszeit. Das Applikationsschema für Imiquimod muss individuell festgelegt werden.

\section{P X/102 Gynäkologie \\ Fallbericht einer cornualen Schwangerschaft in der 13. SsW}

Duscha G., Clavadetscher K., Donadini R., Vogt H.P.

Frauenklinik, Spital Region Oberaargau, Langenthal

Einführung: In 0,25-2\% aller spontan entstandenen Schwangerschaften erfolgt die Implantation nicht eutop. Am häufigsten handelt es sich hierbei um Tubargraviditäten; Raritäten sind cervikale, abdominale, ovariale oder cornuale Schwangerschaften. Die Rate der ektopen Nidationen ist steigend; dies ist unter anderem auf die verbesserte Therapie der Adnexitiden zurückzuführen, wodurch eine Tubenocclusion zwar verhindert, die physiologische Funktion jedoch oft nicht erhalten werden kann. Im Zuge des brei- 
ten Einsatzes artifizieller Reproduktionsmethoden sind auch heterotope, d.h. an zwei Orten angelegte Schwangerschaften häufiger geworden, bei denen die cornuale Lokalisation in 3-4\% der Fälle auftritt. Bei den oft fulminanten Verläufen kann die rechtzeitige Diagnose und rasche Therapie für die Schwangere von vitaler Bedeutung sein. Kasuistik: Wir beschreiben den Fall einer 38-jährigen VIIG IVP mit spontan entstandener Schwangerschaft, die sich in der $12+1$ Amenorrhoewoche mit plötzlich aufgetretenen krampfartigen Unterleibsschmerzen präsentiert. Eine Woche zuvor war vom betreuenden Gynäkologen ein nicht näher spezifizierter ungewöhnlicher Implantationsort bemerkt worden. Anamnestisch ergaben sich neben 4 Spontangeburten sowie 2 Aborten keine Besonderheiten. Bei der sich im Prä-Schockzustand befindlichen Patientin wurde nach ultrasonographischer Verdachtsdiagnose einer rechts cornual gelegenen subrupturierten vitalen Schwangerschaft eine Notfalllaparotomie durchgeführt. Bei massivem Hämatoperitoneum aufgrund eines Durchbruchs von Chorionzottengewebe mit unstillbarer arterieller Blutung an mehreren Stellen musste eine Hysterektomie erfolgen. Postoperativ wurde der Blutverlust mit 3 Erythrozytenkonzentraten substituiert. Die nur langsam genesende und stark traumatisierte Patientin wurde anschliessend einer psychiatrischen Krisenintervention zugeführt. Schlussfolgerungen: Bei Schwangeren mit erhöhtem Risiko für einen ektopen Implantationsort muss auch bei intrauterin lokalisierbarem Feten sorgfältig ausgeschlossen werden, dass es sich um eine seltene cornuale Gravidität handelt. Bei entsprechender Symptomatik sollte auch bei anamnestisch unauffälligen Patientinnen diese Diagnose immer in Betracht gezogen und bei Bedarf abgeklärt werden. Die Sonographie ist hierfür als weithin verfügbares und adäquates Mittel unverzichtbar.

\section{P X/103 Gynäkologie \\ Predictive Value of Resection Margin for Abnormal PAP Smear after Conisation for Cervical Intra-Epithelial Neoplasia (CIN): Preliminary Data}

Sommaruga P. ${ }^{\mathrm{a}}$, Schwartz D. ${ }^{\mathrm{a}}$, Pelte M.F. ${ }^{\mathrm{b}}$, Dubuisson J.B. ${ }^{\mathrm{a}}$ VIastos A.T. ${ }^{\mathrm{a}}$

aDepartment of Obstetrics and Gynecology,

${ }^{b}$ Department of Pathology, Geneva University Hospital

Introduction: Cone margin status has been reported to be the most important predictor of recurrent disease in patients with cervical intra-epithelial neoplasia. We attempted to evaluate free resection margins in cervical conisation specimens as prognostic markers in our population by investigating the statistical association between clear and unclear resection margins of cervical cones and the incidence of abnormal PAP smears. Material and Methods: Retrospective chart reviews from 2000 to 2002. 250 women underwent a conisation for CIN during this period in our institution. $5(2 \%)$ patients were diagnosed with CIN I (2\%), $30(12 \%)$ with CIN II and 215 (86\%) with CIN III. Loop electrocoagulation excision procedure (LEEP) was performed in 247 (97.8\%) patients, the rest of them, 3 patients, were treated with cold knife $(1.2 \%)$. Results: Mean age was 35.7 years (19-77). Hystological analysis showed clear margins in $184(73.6 \%)$ patients and only $66(26.4 \%)$ patients had positive margins (PM), 29 cases $(43 \%)$ were endocervical PM and $41(62 \%)$ cases exocervical PM. After 6 months follow-up PAP smear was performed, 226 (50.4) patients had normal results. Among the 66 patients with PM, 61 (92\%) had normal PAP smear, and $3(4.5 \%)$ showed low-grade dysplasia and 2 were diagnosed with atypical squamous cells of undetermined significancy.
Conclusion: In our material there was no direct relation between free margins and PAP smear results. According to the literature, free resection margin is not an optimal prognostic criterion for recurrence. The search for new prognostic markers for high-risk cases will permit to give adequate therapy and to avoid over-treatment for low-risk groups.

\section{P X/104 Gynäkologie Ulcus vulvae acutum (UVA)}

Ribordy L. ${ }^{\mathrm{a}}$, Abraham S. ${ }^{\mathrm{b}}$, Caubet J.-C. ${ }^{\mathrm{c}}$, Dubuisson J.-B. ${ }^{\mathrm{a}}$, Vlastos A.-T. ${ }^{\text {a }}$

${ }^{a}$ Clinique de Gynécologie, Département de Gynécologie et d'Obstétrique, et Départements de ${ }^{b}$ Dermatologie et

'Pédiatrie, Hôpitaux Universitaires de Genève

Introduction: L'UVA est un diagnostic rare, observé le plus souvent chez l'adolescente. Ce diagnostic différentiel est à garder en mémoire lorsque Herpès Simplex virus (HSV), la cause la plus fréquente d'ulcères génitaux chez la femme, est exclu. Case clinique: Jeune fille de 14 ans, cycles réguliers, virgo, sans aucun contact sexuel, se présente aux urgences de gynécologie avec des ulcérations vulvaires douloureuses. On relève dans l'anamnèse un état grippal depuis quelques jours traité par anti-inflammatoires non stéroïdiens et un sirop antitussif. L'anamnèse relève des aphtes buccaux banaux occasionnels, mais aucune autre lésion cutanée. Les examens paracliniques sont négatifs pour: l'HSV, le Chlamydia trachomatis, la syphilis et l'Ebstein-Barr virus (EBV). De même, la FSC, la CRP, les fonctions rénale et hépatique sont normales. L'examen histologique montre une inflammation aiguë nécrosante sans agent pathogène aux colorations Gram et Grocott. La patiente est hospitalisée afin d'assurer l'antalgie et une sonde urinaire à demeure est posée. Le traitement consiste en une corticothérapie qui a permis une évolution favorable de l'affection et la sortie de la patiente avec un status parfaitement normal au 5ème jour. Discussion: L'HSV, la syphilis, la maladie de Behcet, la réaction toxidermique sont les principales causes d'ulcères génitaux. Les lésions de l'UVA sont d'apparition rapide et l'on retrouve fréquemment une virose dans les jours précédents. Une infection à EBV a été décrite comme cause potentielle. D'autres viroses peuvent provoquer ces mêmes lésions comme dans le cas reporté où un virus de type grippe est suspecté. Le mécanisme pathologique exact est pour l'instant mal connu. Conclusion: L'UVA est une pathologie rare et mal connue, dont il faut se souvenir, car il ne faut pas la méprendre avec une lésion pouvant évoquer un abus sexuel, par exemple.

\section{P X/105 Gynäkologie \\ Interaction mifépristone et traitement anti-épileptique. Rapport de 2 cas cliniques}

Polli C. ${ }^{\mathrm{a}}$, Lourenco A.-P. ${ }^{\mathrm{a}}$, Sebo $P^{\mathrm{b}}{ }^{\mathrm{b}}$, de Ziegler $D .^{\mathrm{a}}$

aDépartement de Gynécologie et d'Obstétrique,

${ }^{b}$ Centre d'Information Clinique et de Pharmacovigilance,

Hôpitaux Universitaires de Genève

Introduction: Le mifépristone (Mifégyne ${ }^{\mathrm{TM}}$ ), un anti-progestérone, est utilisé pour l'interruption médicamenteuse de grossesse (IMG) en Suisse depuis Novembre 1999. Sa métabolisation est hépatique par le cytochrome P450 3A4. Cas cliniques: En Octobre 2004 une patiente de 39 ans, multipare, a présenté une demande d'IMG à 6 0/7 semaines d'aménorrhée. Elle était connue pour une épilepsie traité par Luminal TR (phénobarbital) $200 \mathrm{mg} / \mathrm{j}$. La 
patiente a été traitée selon le protocole classique (mifepristone $600 \mathrm{mg} \mathrm{J} 1$ et misoprostol $800 \mu \mathrm{g}$ p.o. J3). Le contrôle 2 semaines plus tard a montré une grossesse évolutive. Une deuxième patiente de 32 ans, primipare, s'est présentée au mois de Novembre 2004 avec la même demande, à $60 / 7 \mathrm{sem}$. Cette patiente présentait une épilepsie traitée par Keppra TR (lévétiracétam ) 1000 mg/j, Lamictal TR (lamotrigine) $200 \mathrm{mg} / \mathrm{j}$ et Seropram TR (citalopram) $20 \mathrm{mg} / \mathrm{j}$. Elle a été prise en charge selon le même protocole et le contrôle échographique de 2 semaines a montré une grossesse évolutive. Les deux patientes ont subi une aspiration curetage. Discussion: Le taux d'échec reporté de l'IMG dans la littérature avec ce protocole est de 5 dont 1 a $2 \%$ de grossesses évolutives. Des études ont montré que la dose circulant de mifépristone est la même après traitement avec 100 à $800 \mathrm{mg}$ mais que $50 \mathrm{mg}$ de mifépristone est une dose inefficace. Le phénobarbital est un puissant inducteur enzymatique du P450 et il pourrait diminuer la dose circulante de mifépristone en augmentant son catabolisme. Ceci pourrait motiver l'échec de l'IMG. Concernant le lamotrigine et le lévétiracétam ils ne sont pas des inducteurs significatives des enzymes hépatiques. La biotransformation du citalopram est catalysée par le CYP3A4. Le citalopram est un inhibiteur négligeable des enzymes hépatiques et son interférence avec l'efficacité du mifepristone doit être minime. Conclusion: Notre observation nous indique que des traitements de type inducteurs enzymatiques pourraient agir en diminuant l'efficacité du mifépristone dans l'IMG. Autres traitements (lamotrigine et le lévétiracétam) ont moins d'effets in vitro mais des études concernant les interactions avec le mifépristone devront se réaliser pour répondre à la question définitivement.

\section{P X/106 Gynäkologie \\ Portage cervical bactériologique asymptomatique avant la pose d'un dispositif intra-uterin}

Jauch Klein T., Lourenco A., de Ziegler D.

Département de Gynécologie et d'Obstétrique, Hôpitaux Universitaires de Genève

Introduction: Le but de cette étude est d'évaluer la prévalence du portage cervical bactériologique chez des patientes asymptomatiques avant la pose d'un dispositif intra-utérin (DIU). Matériel et méthodes: Collection des données de 138 patientes ayant bénéficié de la pose d'un DIU dans notre service de janvier 2004 à décembre 2004, totalisant 143 poses. Analyse des prélévements bactériologiques cervicaux effectués de routine chez toute patiente en prévision de la pose d'un DIU, comprenant une recherche de C. trachomatis par PCR et une recherche de germes aérobes par culture. Analyse des complications infectieuses ou autres suivant la pose du DIU. Résultats: Sur les 138 patientes étudiées, $114(82,6 \%)$ ont eu un dépistage pour $C$. trachomatis et également $114(82,6 \%)$ une recherche de germes aérobes avant la pose du DIU. Les résultats montrent la présence de $C$. trachomatis chez 1 patiente. Douze patientes $(10,5 \%)$ étaient porteuses de streptococcus B, $5(4,4 \%)$ de Staphylococcus aureus, et $6(5,3 \%)$ de germes type entérobactéries $(E$. coli, proteus, enterobacter et entérococcus). Un traitement antibiotique avant ou dès la pose du DIU a été instauré chez 12 patientes $(8,4 \%)$ comprenant la patiente porteuse de $C$. trachomatis et la moitié des patientes porteuses d'autres bactéries, ce qui représente $48 \%$ des frottis positifs. Toutefois, aucune endométrite n'a été décrite chez des patientes ayant eu un frottis positif non traité. L'analyse des complications infectieuses a montré 2 cas $(1,4 \%)$ d'endométrites à strepto B à 10 jours et à 7 semaines après la pose du DIU chez des patientes qui avaient auparavant des cultures négatives. Le DIU a du être retiré dans 13 cas $(9,1 \%)$ en raison de malpositions ( 8 cas), d'endométrite (1 cas), d'actinomycète (1 cas), d'urticaire généralisé (1 cas) et de spotting persistant (1 cas). Il n'y a pas eu de grossesse dans ce groupe de patiente. Conclusions: La prévalence de l'infection à $C$. trachomatis dans cette population est basse et celle du strepto $\mathrm{B}$ correspond à des prévalences décrites dans la littérature (5-20\%). Un dépistage négatif pour le strepto B n'a pas empêché le développement de 2 cas d'endométrites post-insertion de DIU. D'autres études sont nécessaires pour évaluer le rapport coût-bénéfice d'un screening bactériologique cervical avant la pose d'un DIU.

\section{P X/107 Gynäkologie}

\section{Suivi des adolescentes en colposcopie}

Jäger F, Rossier M.-C., Renteria S., De Grandi P., Gerber S.

Département de Gynécologie-Obstétrique, Centre Hospitalier Universitaire Vaudois, Lausanne

Introduction: L'infection à HPV est la maladie sexuellement transmissible la plus répandue parmi les adolescentes. Leur col semble être plus sensible aux infections à HPV. L'infection est également favorisée par des comportements à risque. Cette étude se focalise sur le profil épidémiologique de ces patientes de 13 à 19 ans suivies en colposcopie. Matériel et méthodes: Toutes les colposcopies effectuées au CHUV sont introduites dans une base de données, qui contient des informations sur l' indication, le diagnostic, le suivi et les facteurs de risque. Cette étude reprend les cas de 1997 à 2004 des patientes âgées de 13 à 19 ans. Résultats: Sur une période de 7 ans, 221 patientes de 13 à 19 ans ont été suivies, soit $5 \%$ de la consultation totale. $40 \%(\mathrm{n}=89)$ avaient 19 ans, $45 \%$ $(n=99)$ avaient entre 17 et 19 ans et environ $15 \%(n=33)$ avaient moins de 17 ans. $80 \%$ des patientes examinées consultaient pour une affection cervicale, $13,5 \%$ pour des condylomes accuminés vulvo-vaginaux isolés et le reste pour d' autres affections. La majorité des patientes dans notre étude consultait pour une dysplasie légère (64\% L-SIL, 14\% ASCUS) ou pour d'autres lésions banales sur le col. Seulement 1,3\% présentaient un H-SIL. Le taux des consultations augmentait avec l'âge. Un des cas de H-SIL au frottis cytologique était pourtant trouvé chez une patiente de 16 ans. Le pourcentage de fumeuses était avec plus de 53\% nettement au-dessus de la moyenne pour cette tranche d'âge en Suisse. 10\% des patientes avaient déjà subi une ou plusieurs interruptions volontaires de grossesse et plus de 5\% avaient accouché au moins une fois. Conclusion: La consultation colposcopique comprend un pourcentage faible d'adolescentes (5\%) qui présentent majoritairement des lésions de bas grade. Vu le comportement à risque, un effort d'information devrait être effectué en ce qui concerne à la prévention.

\section{P X/108 Gynäkologie Interruption de grossesse médicamenteuse chez I'adolescente}

Lourenco A.-P., Ibecheole V.U., de Ziegler D.

Département de Gynécologie et d'Obstétrique, Hôpital Cantonal Universitaire de Genève

Objective: Identifier les aspects particuliers de l'interruption médicamenteuse de grossesse (IGM) chez les adolescents depuis son introduction à la Maternité de l'HCUG. Matériel et méthodes: Analyse rétrospective des dossiers cliniques des adolescentes de 12 19 ans demandeuses d'IGM entre le 1 Janvier 2000 et le 30 Décem- 
bre 2004. L'IMG se réalise à la demande de l'adolescente si la grossesse est de $<50$ jours, en absence d'ambivalence ou de contraindications médicales, et pour les $<18$ ans il est nécessaire que les parents soient informés. Le protocole de traitement est de mifepristone (Mifegyne $^{\mathrm{TM}}$ ) $600 \mathrm{mg}$ p.o. J1 suivi d'une hospitalisation de court durée $(4 \mathrm{~h})$ pour prise de misoprostol $\left(\right.$ Cytotec $\left.^{\mathrm{TM}}\right) 400 \mu \mathrm{g}$ per os, avec analgésie à la demande. Une contraception est proposée à la sortie et une visite de contrôle a lieu 2 semaines après. Résultats: Dans cette période 741 adolescentes ce sont présentés pour une interruption de grossesse. L'IGM a été utilisé chez 20\% ( $\mathrm{n}=147)$, avec une augmentation progressif entre l'année $2000(10 \%)$ et 2004 (30\%). L'âge moyen était de 17,8 ans (14-19 ans). Le taux d'expulsion durant l'hospitalisation était de $67 \%$. Trente-huit pour cent des patientes n'ont pas demandé d' analgésie et 38\% ont étaient traités avec du paracétamol uniquement. Un cas d'endométrite était documenté avec une bonne évolution sous antibiotiques et un cas de rétention partielle était traité par du misoprostol per os. Il n'y a pas eu de grossesses évolutives ni retentions de matérielle nécessitent des curetages chirurgicales. Onze pour cent des patientes ne se sont pas présentés au rendez-vous de contrôle. La satisfaction des patientes à était évalué par un questionnaire. 100\% des adolescentes étaient contentes de leur choix de méthode, $95 \%$ choisiraient à nouveau l'IGM et $95 \%$ recommanderaient la méthode à une amie. Conclusions: Dans le groupe de patientes traités et dans les conditions décrites, l'IGM est une méthode efficace, bien toléré et bien accepté par les adolescentes. Il y a un nombre considérable de patientes qui manque le RDV de contrôle. En raison de rares complications potentiellement graves et l'éventuel échec de la méthode, ils nous semble prudent de limiter cette option aux mineures dont les parents sont au courant.

\section{P X/109 Gynäkologie \\ Häufigkeit von Infektionen mit Chlamydia trachomatis an der Frauenklinik Basel in den Jahren 2003 und 2004}

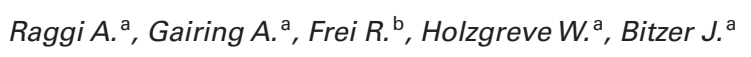

${ }^{a}$ Frauenklinik, Universitätsspital Basel,

${ }^{\text {b} B a k t e r i o l o g i e l a b o r, ~ U n i v e r s i t a ̈ t s s p i t a l ~ B a s e l ~}$

Einführung: Eine Infektion mit Chlamydia trachomatis (C.t.) tritt vor allem bei sexuell aktiven jungen Frauen auf und verläuft oft asymptomatisch. Die Folgen können schwerwiegend sein, wie beispielweise PID (pelvic inflammatory disease), ektope Gravidität oder Sterilität. Das Ziel unserer Untersuchung ist, das Kollektiv der Frauen mit nachgewiesener Infektion mit C.t. hinsichtlich Alter, Parität, Herkunft, Konsultationsgrund und aktueller Antikonzeption zu evaluieren. Material und Methoden: Den Cervix-/ Vaginalabstrich mit Nachweis auf C.t. mittels PCR führen wir in unserer Klinik bei folgenden Indikationen durch: bei asymptomatischen Frauen unter anderem vor geplantem Schwangerschaftsabbruch, vor IUD-Einlage, nach Vergewaltigung, in der Schwangerschaft bei Risikofaktoren, bei Sterilitätsabklärung und nach erfolgter Therapie. Andererseits bei allen Patientinnen, in und ausserhalb der Schwangerschaft, mit Beschwerden wie Fluor, Unterbauchschmerzen oder Blutungsstörung. Wir werteten retrospektiv mittels deskriptiver Statistik Daten aller Frauen mit positivem Abstrichergebnis auf C.t. in den Jahren 2003 und 2004 hinsichtlich oben genannter Kriterien aus. Ergebnisse: Von insgesamt 8208 durchgeführten Abstrichen waren 2,55\% positiv auf C.t. Innerhalb dieser Gruppe war das mediane Alter 25 Jahre, 57\% waren Nulliparae, 43\% der Frauen stammten aus der Schweiz, 16\% aus Osteuropa, $13 \%$ aus der Türkei, 3\% aus Afrika und der Rest aus anderen Ländern. $41 \%$ waren asymptomatisch: $20 \%$ vor Schwangerschaftsab- bruch, $1 \%$ vor IUD-Einlage, 2\% nach Vergewaltigung, 5\% in der Schwangerschaft, $2 \%$ bei Sterilitätsabklärung, 3\% nach erfolgter Therapie und 10\% bei anderen Gründen. 59\% hatten Beschwerden. Die Pille war mit 21\% die häufigste Verhütungsmethode, $8 \%$ verwendeten Kondome und 32\% hatten keine Antikonzeption. Schlussfolgerung: In unserem Kollektiv ist die Prävalenz 2,55\%, mit der relativ höchsten Positivrate bei Patientinnen mit Beschwerden und bei asymptomatischen Frauen vor Schwangerschaftsabbruch. Bei 41 Frauen von den 637 insgesamt durchgeführten Schwangerschaftsabbrüchen in diesen zwei Jahren bedeutet dies eine Entdeckungsrate von 6,4\% und entspricht damit einem sinnvollen Screeninginstrument.

\section{P XI/110 Ultraschall in der Geburtshilfe Cardiac Rhabdomyomas}

Billieux M.-H. ${ }^{\mathrm{a}}$, Antonelli E. ${ }^{\mathrm{a}}$, Da Cruz E. ${ }^{\mathrm{a}}$, VialY. ${ }^{\mathrm{b}}$, Irion O. ${ }^{\mathrm{a}}$

a'Département de Gynécologie et d'Obstétrique, Hôpitaux Universitaires de Genève, 'Département de Gynécologie et d'Obstétrique, Centre Hospitalier Universitaire Vaudois, Lausanne

Introduction: Cardiac tumours are very rare in intrauterine life and early infancy. Their prenatal incidence in screening examinations is estimated as $9 / 100,000$ in a mixed group of high- and lowrisk pregnancies but increases to 1.4-2/1,000 in fetal echographic centres. Most fetal and newborn cardiac tumors are histologically benign. The course in utero depends on the growth of tumors. Large masses can cause hemodynamic obstruction and subsequent heart failure. We report 3 cases of cardiac rhabdomyomas. Case Report: For the 3 cases, a few similar echogenic intracardiac lesions compatible with rhabdomyomas are detected on routine echographic scan at 33, 34 and 35 weeks. For the first case, 3 hyperechogenic nonobstructive tumors are observed on postnatal echocardiographic scan, with a conserved cardiac function, and normal clinical and radiologic observations. For the second case, multiple lesions are observed, with one compromising fetal hemodynamic asking for surgical resection. Cerebral lesions had led to the diagnosis of tuberous sclerosis (TS) and partial regression was noted at age 2 . For the $3 \mathrm{rd}$ case, TS was also diagnosed and complete regression of multiple intracardiac masses was observed at 5 years old. Discussion: Tumors are often detected on routine obstetric sonographic scans in the second half of pregnancy. The most common type of tumor is rhabdomyoma with about $70-80 \%$ of all cardiac tumors, which are often multiple, intracavitary or intramyocardic, associated in more than $50 \%$ of cases to a tuberous sclerosis. Other types of tumors in order of frequency are fibromas, teratoma and myxomas. Findings at ultrasound scan are the presence of rounded, homogenous, hyperechogenic area, that may be difficult to distinguish from another intramural tumour. Partial or complete regression occurs in most newborns with cardiac rhabdomyomas. However, development of intracardiac flow obstruction, alteration of valve function with regurgitation, and accompanying arrhythmias are poor prognostic indicators, and neurological impairment due to tuberous sclerosis is frequently associated.
Abstracts, gynécologie suisse, Lugano, 2005 


\section{P XI/111 Ultraschall in der Geburtshilfe The Giant Umbilical Cord}

\author{
Antonelli E. ${ }^{\mathrm{a}}$, VialY. ${ }^{\mathrm{a}}$, Pfister R. $^{\mathrm{b}}$, Wildhaber B. ${ }^{\mathrm{b}}$, \\ Extermann $P^{\mathrm{a}}$, Irion $\mathrm{O}^{\mathrm{a}}$ \\ Départements de a Gynécologie et d'Obstétrique, et de \\ bédiatrie, Hôpitaux Universitaires de Genève
}

Introduction: A giant umbilical cord is a very rare malformation of the umbilical cord that can easily be diagnosed on prenatal scans. In this report, we describe two cases diagnosed prenatally by ultrasound in which the most prominent prenatal finding was the presence of large umbilical cord cystic masses. Case Report: These two cases were diagnosed at 11 and 20 weeks of pregnancy. The ultrasound examination revealed round, hypoechoic cystic lesions in the umbilical cord near the fetal abdomen. In only one of the case, the cystic lesion was clearly communicating with the fetal bladder. Vesicoallantoic cyst and patent urachus were suspected. After birth, clinical examination of the neonates confirmed large umbilical cords for both cases. For one of the cases, the diagnosis of persistent patent urachus was confirmed immediately after birth. Surgery was performed at day one of life. For the other case, the postnatal ultrasound was inconclusive. The child returned home. One week later, the boy presented again at the hospital for recurrent transparent fluid loss from the umbilicus. The ultrasound revealed a probable communication with the bladder, which was confirmed by cysto-urography. A surgical resection of the persistent patent urachus was performed at 19 days of life with an uneventful postoperative course. Discussion: Umbilical cord cysts develop from the partial or complete absence of obliteration of the allantois or omphalo-mesenteric duct. Congenital patent urachus is an extremely rare condition with an incidence of 1-2.5:100,000 deliveries and is rarely associated with other anomalies, and when isolated, not in relation with chromosomal defects. On prenatal US, the presence of a direct communication between the fetal bladder and the umbilical cord cyst confirms the diagnosis of a patent urachus. This pathognomonic feature, however, is not always apparent prenatally. When detected prenatally, congenital patent urachus should lead to close monitoring and follow-up of the mother and fetus because of a possible compression effect of the cystic mass, particularly at term and during labor, resulting in fetal compromise. If postnatal diagnosis is confirmed, surgical management is usually required.

\section{P XI/112 Ultraschall in der Geburtshilfe Dilatations ventriculaires cérébrales: aspects prénataux}

Kempf Haber M., Beurret Lepori N., VialY.

Département de Gynécologie et d'Obstétrique, Centre

Hospitalier Universitaire Vaudois, Lausanne

Introduction: Déterminer chez les fœtus présentant une dilatation ventriculaire cérébrale (DVC) les anomalies associées, préciser les investigations prénatales et l'évolution post-natale immédiate. Matériel et méthodes: De Janvier 1998 à Décembre 2004, les fœtus présentant une DVC ont été revus, les anomalies foetales associées ont été recherchées ainsi que l'évolution de la grossesse. Résultats: 72 fœtus ont été recensés, $60(83 \%)$ avec une DVC bilatérale et $12(17 \%)$ unilatérale. Cette DVC a été classée comme légère $(10-15 \mathrm{~mm})$ pour $35(48 \%)$, modérée $(16-20 \mathrm{~mm})$ pour 22 $(31 \%)$ et sévère $(>21 \mathrm{~mm})$ pour $15(21 \%)$ fœtus. L'âge moyen du diagnostic était de 27 semaines (14-36 SA). La DVC est restée sta- ble durant le reste de la grossesse chez 48\%, s'est modifiée chez 52\% (diminution pour $24 \%$ de fœtus et péjoration pour $28 \%$ ). Des anomalies associées ont été diagnostiquées par échographie chez 55 fœtus; 14 anomalies extra-cérébrales dont les principales sont: fente labiale, malformation cardiaque, omphalocèle, hernie diaphragmatique, polykystose rénale; et 36 intra-cérébrales dont les principales sont: holoprosencéphalie, agénésie du corps calleux, hypoplasie cérébelleuse, kyste arachnoïdien, sténose de l'aqueduc. Les investigations complémentaires ont été réalisées: caryotype 34 (anormal 7), imagerie par résonance magnétique 13 (DVC toutes confirmées avec 8 autres anomalies), recherches infectieuses 9 (positif 4 pour CMV ou toxoplasmose). L'évolution a montré: 32 interruptions médicales de grossesse, 5 morts in utero, 34 naissances vivantes dont 6 décès en post-natal immédiat. Les détails de l'évolution pré- et post-natales en fonction de la sévérité de DVC seront précisés ultérieurement. Conclusions: Les DVC représentent une pathologie fréquemment rencontrées en prénatal et le pronostic dépend du degré de dilatation et des anomalies associées.

\section{P XI/113 Ultraschall in der Geburtshilfe Hemorragie sous-tentorielle sous-durale in utero}

Heymans Ph. ${ }^{\mathrm{a}}$, Antonelli E. ${ }^{\mathrm{a}}$, Hanquinet $S .{ }^{\mathrm{b}}$, Vial $\mathrm{Y}^{\mathrm{a}}$, Irion $O{ }^{\mathrm{a}}$

Département de ${ }^{\mathrm{a} G y n e ́ c o l o g i e ~ e t ~ d ' O b s t e ́ t r i q u e, ~ e t ~}$

bépartement de Radiologie, Hôpitaux Universitaires de Genève

Introduction: L'hémorragie sous-durale (HSD) prénatale est très rare: 19 cas décrits, dont 1 sous-tentoriel. Les étiologies rapportées incluent les causes traumatiques, troubles de la coagulation, thrombocytopénies et malformations vasculaires. La plupart des HSDs reste toutefois sans explication, compromettant toute prévention. Cas clinique: HSD sous-tentorielle découverte par échographie à 34 semaines dans un contexte de menace d'accouchement prématuré; l'examen révèle une masse hétérogène en fosse postérieure, mesurant $65 \times 55 \times 45 \mathrm{~mm}$, entourée d'une couronne d'aspect hypoéchogène compatible avec du sang et de l'œdème, et comprimant antérieurement les parenchymes cérébral et cérébelleux. Après confirmation par IRM des échographies en deux et trois dimensions, on retient le diagnostic d'hémorragie active de la fosse postérieure, sous-durale, dont l'événement initial remonte à plusieurs jours, associée à un caillot en partie organisé. Les paramètres vélocimétriques au Doppler de l'ACM sont normaux. L'accouchement est pratiqué par césarienne; les IRM ultérieures montrent la progression du processus hémorragique. La compression du tronc cérébral entraînera la nécessité d'intubation transitoire et la nécessité de deux drainages neurochirurgicaux successifs. L'évolution permettra l'extubation et, après 5 semaines en unité de néonatologie, le départ à domicile malgré un pronostic neurologique très réservé. Discussion: En majorité supra-tentorielles, les hémorragies intracrâniennes ont pour siège système ventriculaire, parenchyme cérébral ou espace sous-dural. Les HSD atraumatiques sont exceptionnelles (9 cas décrits); il s'agit de la 2ème situation sous-tentorielle. Aucun facteur parental ni foetal prédisposant ne fut mis à jour, allo-immunisation anti-plaquettaire et anomalies vasculaires comprises. La localisation du saignement et l'étendue du processus compressif constituent des facteurs prédictifs de l'évolution, bien que la corrélation directe soit délicate. Le pronostic de l'HSD anténatal est sombre, comparé à celui de l'hémorragie intraventriculaire, et son issue à long terme très aléatoire; une origine hémorragique sous-tentorielle l'aggrave encore. 


\section{P XI/114 Ultraschall in der Geburtshilfe Pränatale Diagnostik bei einem Feten mit Diastematomyelie und Tethered Cord}

Struben H. ${ }^{a}$, Holzgreve W. ${ }^{a}$, Visca E. ${ }^{a}$, Scheibner K. ${ }^{a}$, Lücking M. ${ }^{\mathrm{b}}$,Tercanli S. ${ }^{\mathrm{a}}$

aUniversitätsfrauenklinik Basel, bUniversitäts-Kinderspital beider Basel

Einführung: Diastematomyelie ist eine seltene Form okkulter Rückenmarksspaltbildungen. Dabei kommt es zur Bildung von zwei Hemicords, die durch einen knöchernen oder auch bindegewebigen Sporn getrennt sein können (75\% Assoziation zu tethered cord). Die pränatale Diagnostik umfasst einerseits den Ultraschall und ergänzend ein fetales MRT. Indirekte Zeichen im Ultraschall sind: Abweichungen im spinalen Verlauf, kutane Auffälligkeiten, Erweiterung des Spinalkanals, echogene Raumforderung im Spinalkanal. In 90\% der Fälle treten Anomalien der darüberliegenden Haut auf, wie z.B. Hypertrichose oder Angiome. Diese werden i.a. erst postpartal erkannt. Falldarstellung: Die 20-jährige Gravida I, Para I stellte sich erstmalig in der $22+1$ SSW vor. Hyperthyreose eingestellt mit Carbimazol. Im Ultraschall zeigte sich eine dezente Erweiterung im Bereich der LWS mit geringer Aufweitung des Spinalkanals Höhe L2-L3 sowie Darstellung eines knöchernen Sporns. Normale Beweglichkeit der Extremitäten, kein Nachweis einer Spina bifida aperta oder anderer Fehlbildungen. Die Amniozentese zeigte einen unauffälligen Karyotyp, sowie normale FW-AFP- und ACHE-Werte. Durchführung eines fetalen MRT mit Bestätigung des sonographischen Befundes. Interdisziplinäre Beratung der Patientin mit Besprechung der Risiken, insbesondere tethered cord und Differentialdiagnosen. Bei der Verlaufskontrolle fiel in der 30. SSW in Höhe der Erweiterung eine streifige hyperdense Struktur auf, entsprechend Haaren. Diese Struktur blieb bis zur $37+0$ SSW nachweisbar. Spontangeburt eines Mädchens in der $40+1$ SSW, unauffällige Primäradaptation. Auf Höhe des Defekts fand sich die schon im Ultraschall entdeckte Hypertrichose, Analreflex nicht auslösbar, ansonsten unauffälliger neurologischer Untersuchungsstatus. Im postpartal durchgeführten Ultraschall und MRT Diagnose einer Diastematomyelie L1-L3 mit tethered cord, Hydromyelie und Conustiefstand. Schlussfolgerung: Soweit uns bekannt ist, wurde bisher noch nie über eine Visualisierung der Hypertrichose über dem Defekt im Ultraschall berichtet. Da eine Hypertrichose über Spaltbildungen sehr häufig ist, sollte bei sonographisch bestehendem Verdacht genaues Augenmerk auf diesen Bereich gerichtet werden.

\section{P XI/115 Ultraschall in der Geburtshilfe \\ Diagnostic échographique des hyperéchogènicités hépatiques fœtales: Signification clinique et prise en charge}

Gucciardo L. ${ }^{\mathrm{a}}$, Antonelli E. ${ }^{\mathrm{a}}$, VialY. ${ }^{\mathrm{b}}$, Irion O. ${ }^{\mathrm{a}}$

aDépartement de Gynécologie-Obstétrique, Hôpitaux Universitaires de Genève, 'Département de GynécologieObstétrique, Centre Hospitalier Universitaire Vaudois, Lausanne

Introduction: La physiopathologie, la signification clinique ainsi que le pronostic des hyperéchogènicités hépatiques fœtales sont encore aujourd'hui un sujet de controverse. Matériel et méthode: Une revue rétrospective de 5 cas a été effectuée. Le diagnostic d'hyperéchogènicités hépatiques fotales a été posé lors d'exa- mens échographiques de routine, entre 22 et 24 semaines de gestation. Pour 4 fœetus, l'évolution et le suivi néonatal ont permis de conclure au caractère isolé de ces images hyperéchogènes. Le dernier cas associait des images hépatiques hyperéchogènes, une fente labio-palatine ainsi qu'un hydrops. Le caryotype effectué a révélé une trisomie 13. Résultats: Les hyperéchogènicités hépatiques fœtales se divisent en deux types: le type 1, parenchymateuses isolées, et le type 2, diffuses parenchymateuses et péritonéales. Ces dernières résultent le plus souvent d'une péritonite méconiale secondaire à des phénomènes intestinaux obstructifs. Les vraies images hyperéchogènes parenchymateuses sont plus rares. Les infections transplacentaires de type varicelle, toxoplasmose, rubéole, cytomégalovirus ou herpès peuvent en être à l'origine. Plus rarement des accidents thrombotiques hépatiques entraînent l'apparition d'hyperéchogènicités de type 1 . Des processus tumoraux hépatiques comme l'hépatoblastome ou le neuroblastome se manifestent, dans certains cas, sous forme de masse parenchymateuse calcifiée. Conclusions: Pour ce qui est des hyperéchogènicités parenchymateuses isolées, la signification clinique n'est toujours pas déterminée avec précision. Une association fréquente avec d'autres anomalies morphologiques doit être exclue par un examen échographique détaillé. Des sérologies maternelles à la recherche d'une infection maternelle seront effectuées. Enfin une prévalence plus importante d'aneuploïdies permet de proposer légitiment une amniocentèse. Un échantillon du liquide amniotique sera mis en culture afin d'identifier un passage transplacentaire d'un agent infectieux maternel.

\section{P XI/116 Ultraschall in der Geburtshilfe \\ Bedeutung der pränatalen Nierensonografie anhand eines Fallbeispieles eines Neugeborenen mit vesikouretralem Reflux und Hudge Divertikel der Harnblase}

Amann E., Remsei G., Zimmermann U., Hess Th., Müller R.C.

Frauenklinik Kantonsspital Winterthur

Einführung: Durch regelmässige nicht nur beim Organscreening (20.-23. SSW) durchgeführte Nierensonografie lässt sich das Handling von Pathologien postpartal durch eine frühzeitige interdisziplinäre Betreuung (Pädiatrie, Radiologie, Chirurgie) optimieren. Dies möchten wir anhand eines Fallbeispieles eines Neugeborenen (Knabe) mit vesikouretralem Reflux Grad II-III rechts und Blasendivertikel darstellen. Material und Methode: Fallbeispiel: 22-jährige I-G, I-P in der 34. SSW sonografischer Nachweis einer zystischen Struktur im linken Unterbauch beim Embryo. In der 35. SSW sonografisch Verdacht auf vesikouretralen Reflux (VUR) rechts mit wechselnd unauffälliger Niere und Hydronephrose bis $9 \mathrm{~mm}$ rechts mit grosser Harnblase von $52 \times 24 \mathrm{~mm}$ und verdickter Harnblasenwand. Am 05.09.04 problemlose Spontangeburt eines Knaben. Am 10. Tag postpartal sonografische Darstellung einer flüssigkeitsgefüllten Raumforderung rechts dorsolateral der Harnblase. Am 07.11.04 Miktions-Cystourogramm (MCUG) mit Darstellung eines parauretralen Divertikels. Antibiotische Prophylaxe prä- und postoperativ. Am 26.11.04 Ureterocystoneostomie(UCN) rechts und Blasendivertikelabtragung. Die klinische Nachkontrolle durch den Kinderchirurgen zeigt einen positiven Verlauf. Postoperative Ultraschalluntersuchung und MAG-3-Szintigrafie zur Verlaufskontrolle vorgesehen. Ergebnisse: Bezogen auf unser Fallbeispiel gute Ergebnisse beim Neugeborenen. Anschauliches Beispiel zur Darlegung der Bedeutung der pränatalen Nierensonografie. Diskussion: Bedeutung der sonografischen Beurteilung der Nieren
Abstracts, gynécologie suisse, Lugano, 2005 
ausserhalb des Organscreenings, z.B. beim Eintritts-Ultraschall im Gebärsaal, Biometrien. Ein «kurzer Blick» auf die Morphologie der Nieren sollte bei jedem Ultraschall erfolgen und ist unserer Meinung nach den, fast zu vernachlässigenden, Mehraufwand wert, wenn sich dadurch gravierende Pathologien darstellen lassen. Nicht allein auf Durchmesser des Nierenbecken-Kelchsystems achten, wichtig auch die Dynamik des Uro-Genitaltraktes zu beachten. Diskussion anhand von Bildmaterial aus der prä- und postpartalen Diagnostik unseres Fallbeispieles.

\section{P XI/117 Ultraschall in der Geburtshilfe Bedeutung der Plazenta-Sonographie}

\author{
Baumann M., Lanz S., Schneider H., Raio L.
}

Universitätsfrauenklinik, Inselspital Bern

Einführung: Die Einführung der Sonographie in der Geburtshilfe machte erstmals die Lokalisierung der Plazenta möglich. Grannum versuchte bereits 1979 Bild und Physiologie zu korrelieren, indem er von der Echostruktur der Plazenta auf den Reifegrad des Fetus schliessen wollte. Heute sollte es möglich sein, durch eine differenzierte sonographische Analyse der Plazentamorphologie eine frühzeitige Selektion von Schwangerschaften durchzuführen, denen spezifische, durch die Plazenta verursachte Komplikationen drohen. Material und Methoden: Es wird eine Serie von sonographisch auffälligen Plazentae präsentiert. Die Arbeit ist nicht systematisch, sondern eine Demonstration von unterschiedlichen Fällen. Auf die spezifischen Implikationen für die jeweilige Schwangerschaft wird kurz eingegangen. Ergebnisse: Gruppe 1 besteht aus Plazentae, welche durch lokalisierte oder generalisierte echoleere Bereiche charakterisiert sind. Dazu gehören Blasenmolen und die noch seltenere mesenchymale Plazentadysplasie. Gruppe 2 stellen plazentare Tumore, insbesondere Chorangiome mit den typischen Komplikationen. Gruppe 3 umfasst übermässig dicke Plazentae wie z.B. jellylike Plazenta oder Megaplacentae. In dieser Gruppe spielt u.a. die Plazentalokalisation eine wichtige Rolle. In Gruppe 4 werden Plazentae präsentiert, welche in das Myometrium einwachsen, namentlich Plazenta increta und percreta. Die Inzidenz solcher Einnistungstörungen nimmt mit zunehmender Sectiorate zu. Gruppe 5 beinhalten Fälle mit Nabelschnurinsertionsanomalien wie velamentöse Insertion und Vasa previa. Spezifisches outcome assoziert mit jeder Gruppe wird präsentiert werden. Schlussfolgerungen: Die Plazentasonographie sollte sich nicht auf Lokalisation und Bestimmung des Grannum-Stadium beschränken. Im Interesse einer Früherkennung von Risikoschwangerschaften sollte die Binnenstruktur der Plazenta sorgfältig studiert und die Nabelschnurinsertion dargestellt werden. Einer auffällig dicken, lateral und fundal liegenden Plazenta sollte besondere Aufmerksamkeit geschenkt werden.

\section{P XI/118 Ultraschall in der Geburtshilfe Gemini-Schwangerschaft: Asynchrone Geburt mit einem Intervall von 126 Tagen \\ Hodel M. ${ }^{a}$, Müller R.C. ${ }^{b}$, Schüssler B. ${ }^{a}$

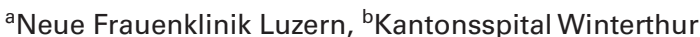

Einleitung: Eine verzögerte Geburt des verbleibenden Fetus bei einer Mehrlingsschwangerschaft ist möglich. Der Wert dieses Prozederes auf das fetale und maternale Outcome bleibt unklar. Kasuistik: Bei der 31-jährigen I G I + II P bestand eine achtjährige primäre Sterilität. Schwangerschaftseintritt unter 4. IVF-Zyklus: di- choriale, diamniote Gemini-Schwangerschaft. $17+3$ SSW: Vorzeitiger Blasensprung Gemini A Hospitalisation KS Winterthur, antiobiotische Abschirmung, sonographisch und 23 + 1 SSW: Verlegung KS Luzern bei portiowirksamer Wehentätigkeit (Cervix $10 \mathrm{~mm}$ ) und V.a. Amnioninfekt (CRP $62 \mathrm{mg} / \mathrm{l})$. 1. Gabe der Lungenreifung (LR) und Gynipral-Tokolyse in Winterthur. 2. LR-Gabe in Luzern. Erneute Antibiose. $23+3$ SSW: Nach abgeschlossener LR Stop der Tokolyse und Geburt des Zwilling A aus II. BEL. Palliative care durch Neo-Team. Exitus letalis des Zwilling A nach 36 h. Hohes Abnabeln und Belassen der Placenta in utero. Atosiban-Tokolyse unverzüglich nach Geburt Zwilling A. Im Verlauf Sistieren der Wehentätigkeit. 24 + 5 SSW: Wiederholung der LR $28+2$ SSW: perzentilengerechtes Wachstum des Zwilling B und normale Fruchtwassermenge. Cervixlänge $41 \mathrm{~mm}$, keine Trichterbildung. Placenta Zwilling A stark verkalkt. Spekulumeinstellung: unauffällige Portio, keine Nabelschnur darstellbar. Verlegung zurück ins KS Winterthur. 29 + 0 SSW: Austritt der Patientin auf eigenen Wunsch bei CK $40 \mathrm{~mm}$, kein Trichter und sonographisch unauffälligem Feten. T + 4: Einleitung mit Cytotec, dann mit Syntocinon. Vakuumextraktion und Geburt eines Mädchens: Gewicht 3000 g, Länge $49 \mathrm{~cm}$, APGAR 8/9/10 NSA-ph 7.24, NSVph 7,35, gute primäre Adaptation. HISTO: Getrennte Zwillingsplacenten (dichorial, diamniot) mit untergewichtiger, vollständig nekrotischer Placenta A und Placenta B mit fokaler dissoziierter Zottenreifungsstörung mit Prävalenz unreifer Endzotten. Diskussion: Eine verzögerte Geburt des verbleibenden Feten kann in Einzelfällen mit einem erstaunlich guten perinatalen Outcome assoziiert sein. Allerdings müssen die Risiken eines intrauterinen Infekts mit möglicher konsekutiver maternaler Sepsis gut bedacht werden. In der Literatur findet sich vorallem in frühen SS-Wochen bei einer verzögerten Geburt von zwei Tagen ein verbessertes kindliches Überleben. Betreffend des SS-Outcomes ist eine ausführliche und empathische Beratung des Elternpaares unerlässlich.

\section{P XII/120 Fötale Medizin Philippinische Famile mit kombinierter Alpha- und Beta-Thalassämie}

Krafft A. ${ }^{\mathrm{a}}$, Dutly $\mathrm{F}^{\mathrm{b}}$, Frischknecht $\mathrm{H}^{\mathrm{b}}{ }^{\mathrm{b}}$,Breymann C. $^{\mathrm{a}}$

${ }^{a}$ Klinik für Geburtshilfe, Universitätsspital Zürich, ${ }^{b}$ Institut für medizinische und molekulare Diagnostik, Zürich

Einführung: Die verschiedenen Varianten der Thalassämie sind die häufigsten monogenen Erkrankungen weltweit. Aufgrund von verstärkter Migration sind Hämoglobinopathie-Varianten auch in der Schweiz mittlerweile keine Seltenheit mehr. Material und Methoden: Wir beschreiben den Fall eines philippinischen Paares mit kombinierter alpha- und beta-Thalassämie, bei dem während der Schwangerschaft ein Hydrops fetalis diagnostiziert wurde. Aufgrund der Ultraschalldiagnostik wurde aus einer Chordozentese mittels isoelektrischer Fokussierung die Diagnose eines Hb-Bart's-Hydrops-fetalis gestellt. Die DNA-Analyse ergab eine «compound» heterozygote $\alpha 0$-Thalassämie major kombiniert mit heterozygoter $\beta 0$-Thalassämie. Ergebnisse: Die 35-jährige, philippinische VI-Gravida und II-Para wurde in der 25. SSW in unsere Klinik zugewiesen wegen vorzeitigen Blasensprunges, vorzeitiger Kontraktionen und Hydrops fetalis. Aufgrund der Ultraschallbefunde wurde eine Chordozentese durchgeführt, die eine schwere fetale Anämie ergab. Isoelektrische Fokussierung und Ionentauscher High-Performance-Liquid-Chromatography (HPLC) zeigten ausschliesslich Hb-Bart's. Sowohl die Mutter als auch der Fetus waren heterozygote Träger der auf den Philippinen häufigen ca. $45 \mathrm{~kb}$ langen Deletion des $\beta$-Gens. Diese Deletion umfasst das 
gesamte $\beta$-Gen und führt zu einer $\beta 0$-Thalassämie. Vor dem Hintergrund der nun vorliegenden Daten konnte beim Feten die Diagnose eines $\alpha 0$-Thalassämie-Hydrops-fetalis gestellt werden. Aufgrund der infausten Prognose entschieden sich die Eltern zur Geburtseinleitung bei $27+4$ SSW. Schlussfolgerung: Für die Diagnostik zeigt das vorliegende Beispiel eindrücklich, dass, falls bei einem Partner eine beta-Thalassämie diagnostiziert wird, der andere zwingend mittels Hämoglobinchromatographie und Hämatogramm abgeklärt werden muss. Kann bei diesem eine normale Hämoglobin-Zusammensetzung nachgewiesen werden, besteht noch kein Grund zur Entwarnung. In diesem Fall ist die Abklärung auf $\alpha$-Thalassämie mittels GAP-PCR und gegebenenfalls Sequenzierung beider $\alpha$-Gene beider Ehepartner dringend indiziert. Gleichzeitig vorliegende, je heterozygote $\alpha$ - und $\beta$-Thalassämien sind insbesondere im südostasiatischen Raum nicht selten.

\section{P XII/121 Fötale Medizin \\ Nabelschnurblut-Banking: \\ Bakterielle und virale Kontamination von Nabelschnurblutspenden}

Nietlispach B., MaierT., Surbek D., Troeger C.,

Meyer-Monard S., Tichelli A., Holzgreve W.

Universitätsspital Basel

Einführung: Die Stammzelltransplantation aus Nabelschnurblut (NSB) ist mittlerweile eine etablierte Therapie-Möglichkeit bei verschiedenen malignen und nicht-malignen Erkrankungen. In der Schweiz wurde unter Koordination von SWISSCORD eine öffentliche NSB-Bank an zur Zeit zwei Standorten (Universitätsspitäler Basel und Genf) gegründet. Dort werden NSB-Spenden entnommen, getestet und bis zu ihrer Verwendung gelagert. Die beiden Banken umfassen mittlerweile 1000 NSB-Spenden. Ziel der Untersuchung ist es, die bakterielle Kontamination der Spenden und die serologischen Untersuchung der Spendermüttern zu analysieren. Material und Methoden: Untersucht wurden NSB-Spendermütter nach Vorselektion im Rahmen der Anamnese (Fragebogen zum Spender-Risiko) respektive der üblichen Schwangerschaftsserologie. Die bakterielle Kontamination wurde mittels aerober und anaerober Blutkulturen geprüft, die virale Kontamination durch Kontrolle des peripheren Blutes der entsprechenden Mütter (Serologie respektive PCR) bestimmt. Ergebnisse: Es wurden $n=759$ NSBSpenden eingeschlossen. Davon waren 30 (4\%) bakteriell kontaminiert. Diese NSB-Spenden wurden nicht ausgeschlossen, das Antibiogramm sollte jedoch vor einer späteren Transplantation bekannt sein. Das Erregerspektrum beinhaltet auch Gruppe B-Streptokokken. Die virale Untersuchung der Mütter ergab: CMV IgG 416 pos. (54,8\%): die Proben werden nicht ausgeschlossen, Lues (VDRL) 2 pos. (0,26\%): diese cords wurden entsorgt, HIV (PCR) 0 pos., HCV (PCR) 2 pos. (0,264\%), Hepatitis B: HbsAg 0 pos., HbcAK (total) 11 pos. (1,45\%), davon anti-HBsAK neg. 0 , pos. (1-100 IU/1) $8(1,05 \%)$, pos. (>100 IU/1) $3(0,4 \%)$. Wenn HbsAg neg. HbcAK pos. und anti-HBsAK $<100$ IU/1 sind, wird das NSB verworfen. Schlussfolgerung: Die bakterielle Kontamination ist vergleichbar mit der anderer grosser NSB-Banken, welche z.T. Raten von 5-6\% angeben. Bei den viralen Serologien betrug die Anzahl der, aufgrund der mütterlichen Kontamination, nicht verwertbaren cords 1,6\%. Unter Nicht- Berücksichtigung der CMV pos. Serologien, bestritten die pos. HBcAK mit 1,45\% den grössten Teil. Die Durchseuchung der Spendermütter mit CMV 55\% entspricht etwa derjenigen der Erwachsenen in Industrieländern. Abschliessend kann gesagt werden, dass sich die NSB-Spendermütter-Selektion der Basler NSB-Bank bewährt.

\section{P XII/122 Fötale Medizin \\ Monochorial-biamniote Gemelli mit selektiver intrauteriner Wachstumsretardierung}

Kuchar I. ${ }^{\mathrm{a}}$, Schneider $H^{\mathrm{a}}$, Dürig $P^{\mathrm{a}}$, Kuhn $P^{\mathrm{a}}$, Nelle M. ${ }^{\mathrm{b}}$, Raio L. ${ }^{a}$

aUniversitätsfrauenklinik und ${ }^{\mathrm{b}}$ Abteilung für Neonatologie, Inselspital Bern

Einleitung: Monochoriale Gemelli weisen eine hohe perinatale Morbidität auf. Im speziellen ist dies bedingt durch das feto-fetale Transfusionssyndrom (FFTS). Eine eher in letzter Zeit erkannte Pathologie, welche in ihrer Ausprägung als Gegenstück zum FFTS gewertet werden kann, stellen Fälle mit selektiver intrauteriner Wachstumsretardierung (SIUWR) dar. Wir berichten über eine Serie von Fällen aus unserem Perinatalzentrum. Material und Methodik: Retrospektiv wurden alle monochorialen Gemelli mit SIUWR der letzten 4 Jahre untersucht. SIUWR wurde definiert als geschätzter Abdomenumfang $<5$. Perzentile eines der Feten und eine Gewichtsdifferenz von $>20 \%$ (geschätztes Gewicht Fetus $(A-B) \cdot 100 / A)$. Das Studienkollektiv wurde mit einem Kollektiv von monochorialen Gemelli ohne Probleme verglichen. Untersucht wurden Geburtsmodus, postnatale Adaptation und neonatologischer Verlauf. Ausgeschlossen waren Fälle mit FFTS und Monoamniote. Resultate: Im erwähnten Zeitraum wurden 72 monochoriale Gemelli gesehen. Davon hatten 10 (13,9\%) einen Feten mit SIUWR (Gruppe 1). Diese Gruppe wurde mit 14 unauffälligen Schwangerschaften (Gruppe 2, 19,4\%) verglichen. Das Gestationsalter bei Geburt war $33,4(25,3-38,0)$ vs. $34(24,2-38,2)$ Wochen $(\mathrm{p}=\mathrm{NS})$. Keine Unterschiede fanden sich auch bei der Sectiorate (80 vs. 78,6\%), 5' Apgar und arteriellem pH. Die Gewichtsdifferenz war in Gruppe 1 grösser $(35,9$ vs. $3,5 \%$; p < 0.001$)$ und es mussten mehr Kinder auf der Intensivstation betreut werden ( 90 vs. $32,2 \%$, p < 0,01). 2 von 20 Kindern in Gruppe 1 starben an Folgen von Fehlbildung und Hirnblutung. Schlussfolgerung: Die Resultate bestätigen, dass Fälle mit SIUWR eine höhere perinatale Problematik aufweisen. Es gibt bislang keine etablierte Therapie. Neben dem expektativen Management gibt es den selektiven Fetozid mittels Koagulation der Nabelschnur bei unmittelbar drohendem Tod des kleinen Feten oder die Laserkoagulation der plazentaren Gefässanastomosen. Solange keine eindeutigen internationalen Richtlinien vorliegen, welche zeigen, dass die invasiven Massnahmen besser sind, werden wir weiterhin expektativ vorgehen.

\section{P XII/123 Fötale Medizin \\ Sonomorphologische Aspekte einer pathologischen Nackentransparenz und ihre Korrelation zur Aneuploidie}

Künzi N., Lanz S., Raio L.

Universitätsfrauenklinik, Inselspital Bern

Einführung: Als morphologisches Kriterium einer pathologischen Nackentransparenz (NT) ist bisher nur das Hygroma colli klar einer Aneuploidie (Turner Syndrom) zugeordnet worden. Andere sonomorphologische Kriterien neben der für die häufigsten Trisomien wurden bisher nicht beschrieben. Ziel dieser Studie ist es, anhand der Morphologie einer pathologischen Nackentransparenz eine Zuordnung zu den häufigsten Trisomien vorzunehmen. Material und Methoden: Retrospektiv wurden alle Fälle der letzten 4 Jahre mit einer NT von $\geqslant 5 \mathrm{~mm}$ nochmals gesichtet und die Sonomorphologie der NT in Abhängigkeit der zugrunde liegenden
Abstracts, gynécologie suisse, Lugano, 2005 
Aneuploidie analysiert. Wir beschränken uns auf die Trisomien 13, 18, 21 und Turner. Ausgeschlossen wurden monochoriale Zwillinge, fehlendes Follow-up, schlechte Bildqualität. Ergebnisse: Es wurden 49 Fälle mit einer NT von $\geqslant 5 \mathrm{~mm}$ gefunden. Davon wiesen 34 (69,4\%) eine Aneuploidie auf (10 Turner, 2 Trisomie 13, 7 Trisomie 18, 12 Trisomie 21, je 1 mit einer Translokation, Triploidie und Mosaik). Alle Feten mit Trisomie 21 zeigen eine kontinuierlich von cranial nach caudal reichende Transparenz mit Maximum im Nackenbereich und zu den Enden jeweils abflachend. Der Inhalt ist echoleer. Teilweise ist ein Hydrops assoziiert. Bei den Turner findet sich die Verdickung lokalisiert im Nacken-/Halsbereich, kragenartig, teilweise scharf begrenzt, gelegentlich mit Septen, selten nach cranial oder caudal auslaufend. Trisomie 13 oder 18 präsentieren ein sehr variables Bild, die Haut ist oft gefältelt, der Inhalt nicht ganz echoleer. Schlussfolgerungen: Trotz der variablen Ätiologie der NT und in Anbetracht der kleinen Fallzahl können bestimmte Erscheinungsbilder den verschiedenen Aneuploidien zugeordnet werden. Um diese Muster zu verifizieren, braucht es weitere prospektive Studien mit grösseren Fallzahlen.

\section{P XII/124 Fötale Medizin \\ Doppler und computerisierte \\ Cardiotokographie in der Überwachung von wachstumsretardierten Feten}

Bénayat M., Raio L., Dürig P., Schneider H.

Universitäts-Frauenklinik, Inselspital Bern

Einleitung: Durch Doppler (arterielles und venöses System) kombiniert mit der computerisierten Cardiotokographie (cCTG)Auswertung kann der Zeitpunkt der Entbindung wachstumsretardierter Feten (IUWR) optimiert werden. Ziel dieser Studie war es, das neonatale Outcome einer Serie von Fällen zu untersuchen, welche mittels dieser Strategie überwacht worden sind. Material und Methodik: Konsekutive IUWR-Feten (Abdomenumfang $<5$. Perzentile), wurden mittels Doppler der Umbilikalarterie (UA PI), Ductus venosus PI (DV PIV) und cCTG [Kurzzeitvariation (KZV)] überwacht. Bei zunehmender Alteration der fetalen Hämodynamik (Zentralisation, AREDF) erfolgte die Hospitalisation. Die Indikation zur Entbindung wurde anhand des fetalen und des mütterlichen Zustandes gestellt. Resultate: In 20 Monaten wurden 36 Fälle aufgenommen. Das Gestationsalter bei Eintritt war 29,8 (23,636), bei Geburt 32,0 $(25,6-37,4)$ Wochen. Die Überwachungszeit betrug 13,9 (0-62) d. Bei Eintritt hatten 41,7\% einen pathologischen Fluss in der UA (19,4\% mit AREDF), 19,4\% waren zentralisiert und 13,9\% zeigten einen erhöhten DV PIV. Präpartal zeigten $58,3 \%$ einen erhöhten UA PI (41,6\% mit AREDF), 36,1\% eine Zentralisation und $30,6 \%$ eine pathologischen DV PIV. Die KZV sank von initial 6,9 $(4-13,8)$ auf 6,2 $(2,1-12,9)$ ab. Die Indikation zur Entbindung war in 25 (69\%) Fällen eine fetale (sich verschlechternde Hämodynamik in $50 \%$, in $24 \%$ pathologische KZV und in $8 \%$ beides). In $95 \%$ erfolgte eine Sectio; alle Kinder wurden auf der neonatologischen Intensivstation behandelt. Das Geburtsgewicht lag bei 1208 (420-2045) g. 16,7\% hatten einen 5' Apgar $<7$ und ein Base Excess >-10 mmol/l wurde in einem Fall gefunden. Die perinatale Mortalität betrug 5,5\%. Schlussfolgerung: Obwohl sich alle Parameter bei diesem Hochrisikokollektiv deutlich verschlechtern im Verlauf, bleibt die perinatale Mortalität und Morbidität im Vergleich zur Literatur niedrig, was sicherlich bedingt ist durch das höhere Gestationsalter bei Geburt.

\section{P XII/125 Fötale Medizin Diskussion von Fehlerquellen nach Chorionzottenbiopsie bei zwei Fällen mit dichorialer Geminigravidität}

Visca E. ${ }^{\mathrm{a}}$,Tercanli S. ${ }^{\mathrm{a}}$, Struben $H^{\mathrm{a}}{ }^{ }$, Miny . $^{\mathrm{b}}$, Holzgreve W. ${ }^{\mathrm{a}}$

a Universitätsfrauenklinik Basel,

bUniversitäts-Kinderspital beider Basel

Einführung: Bei Mehrlingsschwangerschaften ist die frühe Beurteilung der Chorion- und Amnionverhältnisse unabdingbar, insbesondere auch um sicherzustellen, dass alle Feten untersucht werden, wenn eine invasive Diagnostik vorgesehen ist. Kasuistiken: Bei einer 38-jährige Gravida IX, Para IV, St. n. 4 Aborten und 1 betroffenen Kind mit Cystischer Fibrose in der Anamnese wurden mit $11+5$ SSW bei dichorialer diamnialer Zwillingsschwangerschaft eine CVS durchgeführt. Die Chromosomenanalyse war mit 46,XY für beide Feten unauffällig. Der 2. Zwilling war homozygot betroffen von der Cystischen Fibrose. Nach Vorlage der Ergebnisse konnte mit 15 SSW trotz genauer Festlegung mit Dokumentation der fetalen und plazentaren Positionen vor der CVS der betroffene Fetus nicht mehr identifiziert werden, sodass eine Amniozentese mit Indigocarmingabe zur Feststellung der Identitäten durch Mikrosatelliten erfolgen musste. Bei einer weiteren 37-jährige Gravida II, Para I mit dichorialer diamnioter Zwillingsschwangerschaft wurde ebenfalls eine CVS wegen einer Radiusaplasie beim 2. Feten mit $12+6$ SSW durchgeführt. Der Karyotyp beider Feten war 46,XY sodass ebenfalls eine Amniozentese erforderlich wurde. Die Monozygotie wurde durch Mikrosatelliten bestätigt. Diskussion: Die Chorionzottenbiopsie (CVS) ist eine etablierte pränatale Methode zur Abklärung von Chromosomenstörungen und molekulargenetischer Erkrankungen. Neben dem bekannten Eingriffsrisiko ist bei einer Zwillingsschwangerschaft das Risiko einer Kontamination mit Zellen vom anderen Feten ein nicht zu unterschätzendes Risiko. Fehlen sonographische Marker oder handelt es sich um eine monozygote Geminigravidität, ist eine sichere Identifizierung der Individuen ggf. nicht möglich. Daher kann eine Repunktion mit am Ende insgesamt 4 oder mehr Eingriffen resultieren. Schlussfolgerungen: Um Eingriffsrisiken und Fehldiagnosen vorzubeugen, sollte die Indikation zu einer CVS bei Zwillingsschwangerschaften sehr streng indiziert werden. Die zusätzliche Abklärung durch Mikrosatelliten ist eine adäquate Ergänzung, um die Zygotie und Differenzierung der Individuen zu sichern. Diese Risiken sind ferner mit Eltern vorab zu diskutieren.

\section{P XII/126 Fötale Medizin Early/Onset Autosomal Dominant Polycystic Kidney Disease. Case Report}

Liberek C., Ibecheole V., Billieux M.-H., Extermann P., Irion O.

Department of Obstetrics and Gynaecology, University Hospital of Geneva

Introduction: La maladie polykystique rénale autosomique dominante est une affection fréquente, l'incidence est de 1/1000. Environ $2 \%$ des porteurs du gène présentent une forme précoce. A ce jour, 83 cas d'apparition précoce ont été rapportés dans la littérature. Cas familiale: Le premier fotus présente à la 20ème semaine de grossesse des reins de taille augmentée hyperéchogènes. Le caryotype est normal. L'évolution est défavorable avec apparition d'un oligoamnios. Une ITG est pratiquée à la 23ème semaine de 
grossesse. L'examen histologique conclut à une maladie rénale polykystique de type infantile avec une fibrose hépatique congénitale associée. L'hypothèse postulée par les généticiens est celle d'une polykystose rénale de type adulte avec manifestation prénatale en raison de l'anamnèse familiale (père atteint) avec une probabilité de $50 \%$ de récidive. Le 2ème enfant du couple est sain. Lors de la 3ème grossesse, dès la 16 ème semaine, un oligoamnios et des reins hyperéchogènes sont mis en évidence. Une ITG est pratiquée avec un aspect à l'examen histologique identique a celui du 1er fœtus. La mutation précise de cette famille n'a pas été à ce jour identifiée. Discussion: La polykystose rénale in utéro pose des problèmes diagnostiques. La différentiation entre la forme autosomique récessive et la forme dominante est difficile. Le diagnostic repose non pas sur l'âge d'apparition, la clinique ou l'imagerie rénale, qui peuvent être identiques dans les deux maladies, mais sur l'histologie et les arguments génétiques basés sur les ascendants familiaux. Une anamnèse familiale négative n'exclut cependant pas une forme dominante $(25 \%)$. La forme dominante est génétiquement hétérogène. Le gène PDK1 démontre une grande variabilité dans son expression phénotypique, qui inclut la forme prénatale. Un diagnostic prénatal est possible dans les familles chez qui une mutation précise est identifiée. Conclusion: La mortalité périnatale en cas de polykystose adulte d'apparition prénatale est élevée (43\%). Des complications sont présentes chez $67 \%$ des survivants à un âge moyen de 3 ans. Une forte récurrence des formes précoces est documentée $(45 \%)$ parmi les porteurs du gène dans la descendance.

\section{Video}

\section{130 Video \\ Operative Hysteroskopie bei Status nach Endometriumresektion}

Bratschi H.U.

Sonnenhofklinik Engeried, Bern

Einführung: Bei guter Indikationsstellung sind Versager und dadurch bedingte Zweiteingriffe nach hysteroskopischer Endometriumresektion und Einlage des Levonorgestrel-Hormonimplantates (Mirena ${ }^{\circledR}$ ) selten. Typische Symptome derartiger Versager sind erneute Blutungen und menstruationsähnliche Schmerzen, welche zudem sonographisch oft von einer partiellen, ostiumnahen Hämatometra begleitet sind. In meiner Serie von über 1500 operativen Hysteroskopien mit einer Nachbeobachtungszeit von bis zu 12 Jahren betrug die Häufigkeit derartiger Fälle bisher weniger als 5\%. Material und Methode: Im Film gezeigt wird der Fall einer Patientin, welche 6 Jahre nach Endometriumresektion und Einlage des Levonorgestrel-Hormonimplantates zunehmend über Blutungen und Unterbauchschmerzen geklagt hatte. Der Ultraschall zeigte ein isthmisches Myom im Bereiche der Hinterwand und eine Hämatometra ostiumnah beidseits bei tiefsitzendem LevonorgestrelHormonimplantat. Die Patientin wollte eine Hysterektomie unbedingt vermeiden. Anhand des Films wird sowohl auf die pathologisch-anatomischen Gegebenheiten als auch auf die technischen Besonderheiten einer derartigen Zweitoperation eingegangen. Schlussfolgerungen: Bei Kenntnis der pathologisch-anatomischen Besonderheiten und der entsprechenden Technik kann bei Versagern der Endometriumresektion infolge einer partiellen, ostiumnahen Hämatometra in vielen Fällen durch eine erneute operative Hysteroskopie eine Hysterektomie vermieden werden.

\section{131 Video}

\section{Hymenalatresie: Immer mal wieder eine Überraschung}

Spielmann P., Mislin C., Sarlos D., Schär G.

Frauenklinik, Kantonsspital Aarau

Einführung: Die Hymenalatresie ist die häufigste obstruierende Fehlbildung des weiblichen Genitaltraktes. Davon zu unterscheiden sind die transversen Vaginalsepten von denen man annimmt, dass sie aus einer embryonalen Fehlentwicklung des Müllerschen Ganges hervorgehen. Die Diagnose der Hymenalatresie sollte bereits bei Geburt des Kindes gestellt werden. Familiäre Häufungen wurden beschrieben und sollten anamnestisch eruiert werden. Leitsymptom bei Adoleszentinnen mit Hymenalatresie sind in der Regel zyklische Unterbauchschmerzen und das Ausbleiben der Menarche. Fallbeispiel: Die 13-jährige Jugendliche präsentiert sich mit folgenden Symptomen auf der Notfallstation eines Spitals im Nachbarkanton: akuter Harnverhalt, Übelkeit, Erbrechen, bandförmige Rückenschmerzen, Unterbauchschmerzen. Sonographisch V.a. Retentionsblase und Nierenstauung links. Es erfolgt die Zuweisung auf unsere Pädiatrie und konsiliarisch zu uns. Abdominalsonographisch V.a. retrovesikale ca. $10 \times 8,6 \mathrm{~cm}$ grosse echoarme Zyste wahrscheinlich vom linken Ovar ausgehend, mit dem Uterus kommunizierend. Primär Verzicht auf einen Genitalstatus, da die Patientin anamnestisch virgo und erst 13-jährig ist! Nach $24 \mathrm{~h}$ erneuter Harnverhalt, Resistenz bis 5 Querfinger unter dem Nabel palpierbar, übriges Abdomen weich, kein Peritonismus. Genitalstatus: keine Vagina sondierbar, stark dolente, bläuliche Vorwölbung des Hymens. MRI-Becken mit Raumforderung $(7 \times 8 \times 15 \mathrm{~cm})$ mit kranial direkter Fortsetzung in den nach links verlagerten Uterus. V.a. auf Hämatokolpos. Operativer Eingriff und Ergebnis: Operative Hymenalinzision mit dem Kautermesser. Springbrunnenähnliches Auslaufen des Altblutes. Entleeren der massiv dilatierten Vagina. Erweitern der Hymnenalöffunng mittels Kreuzschnitt und Exzision der vier Quadrantenlappen des Hymens. Setzen von acht Einzelknopfnähten im Uhrzeigersinn zur Fixierung der Vaginalmucosa an den Hymenalring. Schlussfolgerung: Bei adoleszenten Mädchen mit rezidivierenden Unterbauchschmerzen und Ausbleiben der Menarche darf die Hymenalatresie in der Differentialdiagnose nicht fehlen. Zur Untersuchung gehört eine genitale Inspektion, in der Regel mit Traktion, zum Einsehen des Introitus. Es sollte noch sorgfältiger darauf geachtet werden, dass bei allen neugeborenen Mädchen, bei der Erstuntersuchung die Hymenalöffung inspektorisch bestätigt wird.

\section{132 Video \\ Laparoskopische Sigmatransposition zur Behandlung einer Vaginalaplasie beim Meyer-Rokitansky-Küster-Syndrom (MRKS)}

Schnyder G., Hohl M.K., Teufelberger G.

Frauenklinik, Kantonsspital Baden

Einführung: Das MRKS ist die häufigste Ursache einer vaginalen Aplasie mit einem Vorkommen in 1:5000 Geburten. Zur Behandlung hat bereits Frank 1934 eine Methode zur Dehnung des Vaginalsinus beschrieben. Vecchietti hat in den 60er Jahren ein transabdominales Traktionssystem eingeführt, mit dessen Anwendung über mehrere Tage mittels Dehnung eine Neovagina von bis zu $12 \mathrm{~cm}$ Länge hergestellt werden kann. Diese Methode hat sich dank guter Langzeitergebnisse und neuerdings auch möglicher la-
Abstracts, gynécologie suisse, Lugano, 2005 
paroskopischer Präparation durchgesetzt. Trotzdem kann es in seltenen Fällen zu einer Verkürzung der Neovagina kommen. In dieser Situation bietet sich die Methode einer Scheidenherstellung mittels Sigmatransposition an. Dieses Verfahren ist seit bereits fünfzig Jahren bekannt. Nun ist es jedoch im Rahmen der minimalinvasiven Chirurgie möglich, diese Operation ebenfalls laparoskopisch durchzuführen. Methode: Videodemonstration der laparoskopischen Sigmatransposition zur Herstellung einer Neovagina bei einer 35-jährigen Patientin mit MRKS, bei welcher es bei St. $n$. Vecchietti-Operation vor 18 Jahren zu einer Schrumpfung der Neovagina kam. Ergebnis: Nach komplikationslosem postoperativen Verlauf mit rascher Erholung zeigt sich nach Abschluss der Wundheilung eine ausreichend lange Scheide ohne Verengung mit nun wieder normaler Sexualität. Schlussfolgerung: Es handelt sich bei dieser Operationsmethode um ein sicheres Verfahren. Zudem ist damit auf Grund der minimalinvasiven Chirurgie eine nur geringe Morbidität verbunden. Der wesentliche Vorteil dieser Operation besteht darin, dass eine Nachbehandlung, die bei allen anderen Verfahren obligatorisch ist (Dehnung, Spülung, Verwendung von Stents), entfällt und eine Kohabitation bereits 6 Wochen postoperativ möglich ist. Im Vergleich zu den anderen Methoden unterscheiden sich die Kurz- und Langzeitergebnisse nicht und sind mit normaler Sexualität ausgezeichnet.

\section{133 Video \\ Die operative Therapie der tief infiltrierenden Endometriose: Von der Darmresektion zur Ureternaht}

Huber A.W., Lanz S., Raio L., Dreher E., Mueller M.D.

Universitäts-Frauenklinik, Inselspital Bern

Einführung: Die chirurgische Therapie der tief infiltrierenden Endometriose, welche die Ligamenta sacrouterinae, das Septum rectovaginale sowie benachbarte Organe wie Darm, Harnblase oder Ureteren befällt, stellt eine Herausforderung in der operativen Gynäkologie dar. Material und Methoden: Entsprechend der Literatur sind, bei tief infiltrierender Endometriose, die Ligamenta sacrouterinae in ca. $65 \%$ der Fälle, die Vagina in ca. 15\%, der Darm in ca. $10 \%$, die Harnblase in ca. $7 \%$ und die Ureteren in ca. $1,5 \%$ der Fälle befallen. Eine subjektiv zufriedenstellende Symptombesserung kann, bei entsprechendem chirurgischem Verfahren, in über $70 \%$ der Frauen mit tiefer Dyspareunie und in über $60 \%$ mit schwerer Dysmenorrhoe erreicht werden. Die radikal operative Therapie ist gegenüber dem Debulking deutlich effizienter (8\% Restsymptomatik nach radikaler Operation versus 79\% nach Debulking bei Blaseninfiltration) und sollte deshalb angestrebt werden. Ergebnisse: In exemplarisch ausgewählten Ausschnitten von laparoskopischen Operationsvideos werden die verschiedenen Lokalisationen von tief infiltrierenden Endometrioseherden präsentiert und deren laparoskopische Exzisionsmöglichkeiten (Resektion der Ligamenta sacrouterinae, Blasenteilresektion, Ureterteilresektion und Reanastomosierung, Darmresektion usw.) vorgestellt. Schlussfolgerung: Die radikale laparoskopische Resektion von tief infiltrierenden Endometrioseherden ist chirurgisch anspruchsvoll, kann aber meist erreicht werden. 
Abstracts, gynécologie suisse, Lugano, 2005

Römische Zahl = Session. Arabische Zahl = Präsentation. Änderungen vorbehalten.

Abraham, S. P X/104

Agorastos, S. P IX/94

Amann, E. P XI/116

Antonelli, E. FM V/53, P XI/110, P XI/111, P XI/113, P XI/115

Antùnez, L. P IV/47

Arlettaz, R. FM IV/42

Aymon, D. P IV/42

Bannwart, F. P V/50, VI/64

Bass, B. FM III/31, P IX/91

Baumann, M. P XI/117

Beck, G. P I/12, P IV/45, P VII/71

Beer, P. P VII/74

Beffa, V. FM VI/60

Beinder, E. FM I/10, FM IV/42, P VII/76

Belhia, F. P VIII/84

Bénayat, M. P XII/124

Bencaiova, G. P VII/76

Berner, F. P III/36

Berner, M. P IV/46

Bersinger, N.A. FM I/12, P IX/92

Bertschinger, M. P V/50

Beurret Lepori, N. P IX/98, P XI/112

Beutler, G. P V/51

Bianchi-Demicheli, F. P I/11, P I/13, P VII/70

Billieux, M.H. FM V/54, P XI/110, P XII/126

Birkenmaier, A. P IV/44

Birkhäuser, M. FM III/33

Bischof Delaloye, A. P V/58

Bischof, P. FM I/14, FM VI/60, P VI/67

Bitzer, J. P I/14, P I/15, P X/109

Bloch, A. P I/16, P IV/47

Bodmer, C. FM III/33

Boehlen, F. FM V/53

Borisch, B. P V/52

Bork, K. P IX/96

Botta, U. P II/22

Bouchardy, C. P II/22, P V/52, P V/59

Boulvain, M. FM I/13, FM III/32, FM IV/40, FM V/53, P I/10, P I/11, P IV/46, P VII/70

Bourgain, C. P VI/65

Bracher, Ph. P VII/79.1

Brancatelli, G. P VII/71

Bratschi, H.U. P II/26, V 130

Breymann, C. P VII/73, P XII/120

Brown, D. P VI/61, P VI/62

Brülhart, G. P IV/41

Brunisholz, Y. P V/58

Bucher, H.U. P VI/61, P VI/62

Bucher, S. P III/38

Bühlmann, U. P IX/91

Burger, D.A. P IX/90, P V/51

Burger, H. P IX/95
Burkhardt, T. FM IV/41, FM IV/42, FM V/52, P VII/73, P VII/76

Bürki, N. P V/50, P VI/64

Bütikofer, K. P IV/45

Cairoli, A. P VIII/84

Caubet, J.-C. P X/104

Cavenaghi, E. P VIII/80

Chabloz, P. P VII/79.1

Chanson, A. FM I/15

Chappuis, P.O. P V/59

Christoffel, L. P II/23

Clavadetscher, K. P X/102

Csizy-Botond, L. P IV/41

Da Cruz, E. P XI/110

Dällenbach, P. P II/25

Damnon, F. P IX/98

Daniel, F. P IX/97

de Candolle, G. P VI/67

De Grandi, P. P I/17, P V/58, P X/107

De Luca, R. P IV/46

de Moerloose, P. FM V/53

de Ziegler, D. FM I/14, FM III/34, P VI/65, P VI/67, P X/105, $\mathrm{P} X / 106, \mathrm{P} X / 108$

Dedes, K.J. P II/24

Delaloye, J.-F. P V/58, P VII/75

Dellas, A. P III/37

Di Cienzo, G. P VIII/85

Di Santo, S. FM I/12

Diaz, M. P VII/75

Diesch, C.H. P VIII/85

Dombi, V.H. P VIII/82, P IX/94

Donadini, R. P X/102

Dost, F. FM III/35

Dreher, E. FM II/23, FM II/25, FM III/30, FM VI/62, FM VI/64, P II/26, P IX/92, P X/101, V 133

Dubé, M.J. FM II/21, P IX/97

Dubuisson, J.B. FM II/21, P II/20, P II/22, P IX/97, P V/56, $\mathrm{P} X / 103, \mathrm{P} X / 104$

Duc, C. P IV/42

Dürig, P. FM I/11, FM IV/45, P XII/122, P XII/124

Duscha, G. P X/102

Dutly, F. P XII/120

Eberhard, J. P VIII/81

Eberhard, M. FM VI/62, P III/31, P V/57, P X/100

Eggimann, T. P II/26

Eitel, K. P V/57

Epiney, M. FM V/53

Etessamipour, B. P VIII/88

Extermann, P. P XI/111, P XII/126 
Faltin, D.L. FM III/32, FM IV/40, FM VI/61, P I/11, P VII/70

Fasnacht, M. FM V/50, FM V/51

Fässler, S. FM I/11

Fedier, A. P II/21, P II/24

Fehr, M. P V/54, P V/55

Feki, A. FM VI/61, P V/53

Fiche, M. P V/58

Fink, D. FM II/20, FM II/22, FM VI/65, P II/21, P II/24, P II/27, P III/32, III/33, P III/34, P III/36, P IX/93, P V/54, P V/55, P VI/61, P VI/62

Fioretta, G. P V/59

Fleischmann, A. FM II/25

Flori, L. P I/11

Floris, L.A. FM III/32, FM IV/40, P VII/70

Fondop, J.J. FM III/34, P VI/67

Fontana, M. P IV/44

Fournet Irion, N. FM I/14

Frei, K.A. FM I/11

Frei, R. P X/109

Frischknecht, H. P XII/120

Frolik, D. P VII/74

Gairing, A. P IV/40, P X/109

Gardiner-Garden, M. P II/27

Gaudenz, R. P III/37

Geiger, S. P III/37

Geissbühler, V. P VIII/81

Gerber, S. P VIII/84, P X/107

Germond, M. FM I/15

Ghezzi, F. FM I/12

Ghisla, R. FM V/50, FM V/51

Gianoni, A. P II/28

Gloor, B. FM II/25

Grabbert, M. P VII/72

Gross, N. P VI/64

Gucciardo, L. P XI/115

Günter, D. FM VI/64

Günthard, J. FM V/50, FM V/51

Gyr, Th. FM VI/62, P II/26, P II/28, P VIII/87

Hachemane, K. P VIII/80

Hacker, N.F. P II/27

Hagen, B.D. P VII/79

Hagmann, D. FM VI/65

Hallenbarter, C. P III/33

Hanquinet, S. P XI/113

Harpes, P. P IV/43

Hauri, D. FM II/20

Heinzelmann-Schwarz, V.A. P II/27

Henshall, S.M. P II/27

Herrmann, U. P IV/41

Hess, T. FM VI/65, P VII/77, P XI/116

Hetzel, P. P IV/48

Heymans, Ph. P XI/113

Hodel, M. P XI/118

Hoffmann, K. FM IV/44

Hohl, M.K. V 132

Hohlfeld, P. P I/16, P VII/75, P VIII/84

Holzgreve, W. FM VI/63, P I/14, P I/15, P III/30, P IV/40, P IV/44, P IV/48, P VII/78, P VIII/85, P VIII/86, P X/109, $\mathrm{P}$ XI/14, P XII/121, P XII/125

Honegger, Ch. P II/23, P IX/94, P VIII/82

Hornung, R. P VI/61, P VI/62

Hösli, I. P IV/40, P IV/44, PIV/48, P VII/78, P VIII/86

Huber, A.W. V 133

Abstracts, gynécologie suisse, Lugano 2005
Humburg, J. P III/30, P VII/78

Hummel, T. FM IV/43

Hund, M. P VI/69

Hung, B. P IX/90

Huth, S. FM VI/65

Ibecheole, V.U. FM III/34, P VI/67, P XII/126, P X/108

Imesch, P. P II/24

Infanger, E. FM VI/65

Irion, O. FM I/13, FM III/32, FM IV/40, FM V/53, FM V/54, FM VI/61, P I/10, P I/11, P IV/46, P VII/70, P XI/110, P XI/111, P XI/113, P XI/115, P XII/126

Irminger-Finger, I. FM VI/61, P V/53

Jacob, S. FM II/21

Jäger, F. P X/107

Jauch Klein, T. FM I/14, P X/106

John, H. P VIII/82

Kaplan, Z. P IX/91

Kaufmann, M. P VII/74

Kempf Haber, M. P XI/112

Kern, Ch. FM V/54

Kinkel, K. P VI/66

Kleeb, B. P IV/45, P VII/71

Kleinert, D. P V/54

Kloetzer, L. FM V/54

Korte, W. P IX/91

Krafft, A. P XII/120

Krause, E. P VIII/86

Kuchar, I. P XII/122

Kudla, A. P IX/95

Kuen, P. FM V/50, FM V/5

Kuhn, A. FM II/23, FM III/30, P III/35, P IX/96

Kuhn, P. FM I/11, FM IV/45, P XII/122

Künzi, N. P XII/123

Kurmanavicius, J. FM IV/41, FM V/52

Kuschel, S. FM II/24, FM III/35, P III/38

Ladewig, A. P IV/48, P VIII/86

Lanz, S. FM IV/44, FM VI/62, P XI/117, P XII/123, V 133

Lauper, U. P IX/93

Leidi, L. P II/28

Leitner, U. P VII/74

Lengsfeld, D. P X/10

Liberek, C. P XII/126

Limoni, C. FM III/33

Litschgi, M. P III/31, P V/57, P X/100

Locca, Y. P II/20

Lopez, R. P IV/47

Lourenco, A.-P. P X/105, P X/106, P X/108

Lücking, M. P XI/114

Lüscher, K.P. P II/26

Magnenat, D. P I/10

Maier, T. P XII/121

Malek, A. FM I/12

Manegold, G. P VII/78

Mariotta, M. P III/37

Markus, A. P X/100

Matthey, M.-L. P II/26

Matzinger, B. P VI/61, P VI/62

Megalo, A. FM I/15

Merki-Feld, G.S. P VI/69

Meyer-Monard, S. P XII/121

Gynäkol Geburtshilfliche Rundsch 
Miny, P. P XII/125

Mislin, C. V 131

Monod, J.F. P V/58

Montani, M. P IX/93

Montavon, C. P IV/48, P VIII/86

Morales, M.A. FM V/53, FM V/54, P IV/46

Mueller, M.C. V 133

Mueller, M.D. FM I/12, FM II/25, FM VI/62, FM VI/64, P II/26, P IX/92, P X/101

Müller, A. P VI/63

Müller, C. P V/54

Müller, R.C. P VII/77, P XI/116, P XI/118

Najjari, L. P III/38

Nelle, M. P XII/122

Neukomm, C.B. FM IV/45

Neyrond, I. P II/22

Nietlispach, B. P XII/121

Noembrini, B. P II/28

Nogaretti, C. P II/28

O’Brien, P.M. P II/27

Ochsenbein-Imhof, N. FM II/25

Ochsenbein-Kölble, N. FM IV/43,P IV/43

Ortigue, S. P I/13

Otero, M. FM III/32, P I/11, P VII/70

Palacio-Espasa, F. P I/10

Pariès, J. FM V/54

Parziale, L. P IV/47

Pascale, P. P VIII/84

Passi, A. FM I/12

Pauli, E. P V/55

Paulson, R. P VI/65

Pavic, N. P VI/60

Pellegrinelli, J.-M. P IV/42

Pelte, M.F. FM VI/60, P II/20, P II/25, P V/52, P V/56, P VI/66, $\mathrm{P} \mathrm{X} / 103$

Perucchini, D. FM II/20, FM II/22, P III/32, P III/33, P III/34, P III/36

Pfammatter, J.P. FM V/50, FM V/51

Pfander, M. P III/32

Pfister, R.E. FM V/54, P IV/46, P XI/111

Plangger, N. P VIII/83

Pok, J. P VI/64

Polli, C. P X/105

Primi, M.-P. FM I/15

Qayoom, Z. P I/10

Raggi, A. P VIII/85, P X/109

Raio, L. FM I/11, FM I/12, FM IV/44, FM IV/45, P IX/92, P XI/117, P XII/122, P XII/123, P XII/124, V 133

Rapiti, E. P V/59

Rautenberg, O. P VIII/81

Rautenberg, W. P II/23

Reber, G. FM V/53

Reinke, C. P IX/96

Reize, Ch. P I/12

Remsei, G. P XI/116

Renteria, S.-C. P I/16, P I/17, P X/107

Renzi, G. FM I/13

Ribordy, L. P X/104

Richetti, A. P II/28,

Righetti A. FM I/14
Rist, L. P VIII/83

Robyr, R. FM V/55

Rohner, P. FM I/13

Rollini, C. P I/11

Rombaldi, F. P V/56

Romoscanu, I. FM III/34, P VI/65

Rossi Montalbetti, F. P VIII/87

Rossier, M.-C. P X/107

Rubenov, R. P III/34

Safwan, S. P II/28, P VIII/87

Salomon, F. P IX/93

Samba-Nganga, A.B. P IX/97

Sandrine, J. P IX/97

Sangalli, M.R. FM III/32, P VII/70

Santi, A. FM VI/62

Sapin, B. P IX/95

Sappino, A.P. P V/59

Sarlos, D. P VI/68, V 131

Sarp, S. P V/59

Savary, M.-C. P I/17

Schäffer, L. FM IV/42

Schäffer, P. P V/56

Schapira, M. P VIII/84

Schär, G. P VI/68, V 131

Scheibner, K. P XI/114

Scheiner, D. FM II/22, P III/33, P III/34, P III/36

Scherer, M. P IX/90

Scherrer, R. P IX/94

Schilling, J. FM VI/65

Schmid, D.M. FM II/20, FM II/24

Schmid, N. P V/51

Schneider, Ch. P III/32

Schneider, H. FM I/11, FM I/12, FM IV/45, P XI/117, P XII/122, P XII/124

Schneider, N. P IV/42

Schnyder, G. V 132

Schoch, E. P VII/77

Schossig, C. P I/15

Schrack, V. P VII/77

Schrenzel, J. FM I/13

Schubert, H. P V/59

Schuchter, U. P II/21

Schuller, C. FM VI/63

Schulzke, S. P IV/44

Schüssler, B. FM II/24, FM III/35, P XI/118

Schüssler, S. P III/38

Schwartz, D. P X/103

Schwarz, C. FM I/14

Scolyer, R.A. P II/27

Scurry, J. P II/27

Sebo, P. P X/105

Sekarski, N. FM V/50, FM V/51

Senn, A. FM I/15

Sessa, Ch. P II/28

Seydoux, J. P IV/47

Söhnchen, N. P IV/42

Sommaruga, P. P X/103

Sonnenschein, M. P II/26

Spielmann, P. V 131

Spinosa, J.P. P III/35

Spörri, S. P VI/63

Stahel, M.C. P VI/61, P VI/62

Stähler van Amerongen, K. FM II/23

Stalberg, A. FM III/34 
Stallmach, T. P VII/79

Stan, C. FM I/13

Stanczyk, F. P VI/65

Staub, D. P IV/43

Stäubli, A.C. P I/14

Steiner, R. P II/26

Steinmann, H. FM V/50, FM V/51

Stierli, M. P I/12, P IV/45

Struben, H. P IV/48, P XI/114, P XII/125

Stucki, D. P II/26, P VI/63

Surbek, D. FM VI/65, P IV/40, P XII/121

Sutherland, R.L. P II/27

Tasias, D. P II/28

Temgoua, A. P IX/95

Tercanli, S. P IV/48, P XI/114, P XII/125

Terrier, F. P VI/66

Teufelberger, G. V 132

Tichelli, A. P XII/121

Todesco, M. P VII/79

Tolck, P.H. P VIII/80

Tosi, C. P VIII/87

Tresselt, C. P III/31

Treustedt, J. P III/35

Troeger, C. P III/30, P XII/121

Tschudin, S. P I/14, P I/15

Urner, F. FM I/15

Usel, M. P II/22

Ventura, P. FM III/34, P VI/67

Venturelli, S. P IX/93

Venzi, G. P VIII/87

Verkooijen, H.M. P V/59

Vial, Y. P XI/110, P XI/111, P XI/112, P XI/113, P XI/115

Ville, Y. FM V/55
Visca, E. P XI/114, P XII/125

Vlastos, A.-T. FM VI/60, FM VI/61, P II/20, P II/22, P V/52, $\mathrm{P}$ V/53, P X/103, P X/104

Vlastos, G. FM VI/61, P II/20, P II/25, P V/52, P V/53, P V/56, $\mathrm{P}$ V/59, P VI/66

Vogt, H.P. P VII/72, P X/102

von Castelberg, B. FM III/31, P IX/91

von Mandach, U. P VIII/83

von Mering, R. FM IV/43

von Orelli, S. P IX/93, P V/54, P V/55

Von Roten, S. P IX/92

Vuilliomenet, A. P VII/79.1

Waber, H. FM I/11

Warkus, T. P V/58, P VII/75

Weil, A. FM III/32, FM IV/40, P VII/70

Weingartner, M. FM III/31

Weintraub, J. P VI/65

Wenger, J.M. FM II/21

Werner, M. FM II/20, FM II/24, FM III/35, P III/38

Wight, E. FM VI/63, P III/30

Wildhaber, B. P XI/111

Wirthner, D. FM I/15

Wolf, M. P VI/61, P VI/62

Wunder, D.M. FM III/33, P IX/92

Wyss, P. FM VI/65

Yamamoto, M. FM V/55

Yeginsoy, S. P IX/90, P V/51

Zanetti, R. FM VI/63, P IV/44

Zimmermann, K. P VIII/81

Zimmermann, R. FM IV/41, FM IV/43, FM V/52, P IV/43, P VII/79, P VIII/83

Zimmermann, U. P XI/116

Zollikofer, C.L. P VII/77 